University of Trás-os-Montes e Alto Douro

\title{
Ontology-based Procedural Modelling of Traversable Buildings Composed by Arbitrary Shapes
}

Doctoral Thesis in Computer Science By Telmo Miguel Oliveira Adão

Supervisors:

Professor Luís Gonzaga Mendes Magalhães

Professor Emanuel Soares Peres Correia

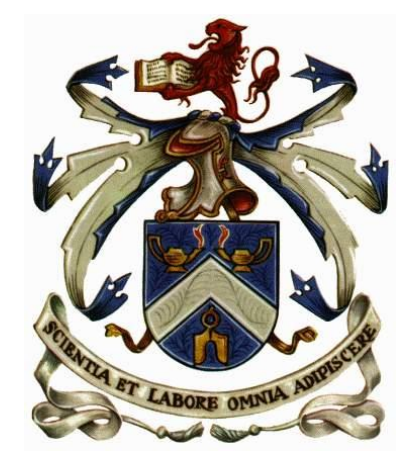

Vila Real, 2015 



\title{
Ontology-based Procedural Modelling of Traversable Buildings Composed by Arbitrary Shapes
}

\author{
By \\ Telmo Miguel Oliveira Adão \\ Supervisor: Professor Luís Gonzaga Mendes Magalhães \\ Co-supervisor: Professor Emanuel Soares Peres Correia
}

Thesis submitted to

UNIVERSITY OF TRÁS-OS-MONTES E ALTO DOURO

in partial fulfillment of the requirements

for the degree of DOCTOR PHILOSOPHY

in Computer Science, accordingly with the provisions of

DR - I A-series, n. ${ }^{\circ}$ 74/2006, March 24th and accordingly with the

Regulation of the Postgraduate Studies of UTAD

DR, 2nd series - Deliberation n. ${ }^{\circ} 2391 / 2007$ 



\title{
Ontology-based Procedural Modelling of Traversable Buildings Composed by Arbitrary Shapes
}

\author{
By \\ Telmo Miguel Oliveira Adão \\ Supervisor: Professor Luís Gonzaga Mendes Magalhães \\ Co-supervisor: Professor Emanuel Soares Peres Correia
}

Thesis submitted to

UNIVERSITY OF TRÁS-OS-MONTES E ALTO DOURO

in partial fulfillment of the requirements

for the degree of DOCTOR PHILOSOPHY

in Computer Science, accordingly with the provisions of

DR - I A-series, n. ${ }^{\circ}$ 74/2006, March 24th and accordingly with the

Regulation of the Postgraduate Studies of UTAD

DR, 2nd series - Deliberation n. ${ }^{\circ} 2391 / 2007$ 

Supervisors:

\title{
Professor Luís Gonzaga Mendes Magalhães
}

Assistant Professor with Habilitation

Department of Information Systems

University of Minho

\section{Professor Emanuel Soares Peres Correia}

\author{
Assistant Professor
}

Engineering Department, Science and Technology School

University of Trás-os-Montes e Alto Douro 

Learn from yesterday, live for today, hope for tomorrow. The important thing is not to stop questioning.

Albert Einstein

To my family and friends

To my soulmate Dércia 



\section{UNIVERSITY OF TRÁS-OS-MONTES E ALTO DOURO \\ $\mathrm{PhD}$ in Computer Science}

The members of the jury recommend to the University of Trás-os-Montes e Alto Douro the acceptance of the thesis entitled "Ontology-based Procedural Modelling of Traversable Buildings Composed by Arbitrary Shapes" accomplished by Telmo Miguel Oliveira Adão in partial fulfillment of the requirements for the degree of Doctor Philosophy.

December 2015

Jury President: Professor Vitor Manuel de Jesus Filipe,

Associate Professor with Habilitation of the University of

Trás-os-Montes e Alto Douro

Jury Members: Professor Adérito Fernandes Marcos,

Full Professor of the Aberta University

Professor António Augusto de Sousa,

Associate Professor of the Engineering Faculty of the University of Porto

Professor Luís Paulo Peixoto dos Santos,

Assistant Professor of the Engineering School of the University of Minho

\section{Professor Nuno Carlos Sousa Rodrigues,}

Adjunct Professor of the School of Technology and Management of the Polytechnic Institute of Leiria 
Professor Luís Gonzaga Mendes Magalhães,

Assistant Professor with Habilitation of the Department of

Information Systems of the University of Minho

\section{Professor Emanuel Soares Peres Correia,}

Assistant Professor of the Engineering Department, Science and

Technology School of the University of Trás-os-Montes e Alto Douro 


\title{
Ontology-based Procedural Modelling of Traversable Buildings Composed by Arbitrary Shapes
}

\author{
Telmo Miguel Oliveira Adão
}

\author{
Submitted to the University of Trás-os-Montes e Alto Douro \\ in partial fulfillment of the requirements \\ for the degree of Doctor of Philosophy
}

\begin{abstract}
D virtual models of buildings are commonly used in areas such as architecture and video games to preview a house project and to populate a virtual scenario, respectively. Traditionally, the production of these models requires highly skilled manpower and a considerable amount of time. To address this issue, many researchers have developed semi-automatic techniques to produce virtual models expeditiously. These procedural techniques provide different ways of generating buildings, including interiors and outer facades, to serve several purposes (e.g., content generation for video games or archaeological reconstruction). However, the existing techniques focusing on building interiors usually only support the generation of floor plans constrained by regular shapes or contour polygons obtained from rectangles sets. At the same time, the possibility of modelling interior rooms through the specification of its constraint walls remains poorly explored. Moreover, most of the existing procedural generation solutions are guided by complex grammars concerned with geometrical aspects or semantic structures that fit specific project requirements, apparently disregarding the established standards for virtual urban environments, specifically, CityGML.
\end{abstract}

To overcome the noted issues, a novel procedural modelling methodology is proposed in this thesis, one that produces virtual models of buildings, including exteriors outlined by arbitrary shapes and interiors formed by convex polygons. Methodology's regulation is provided by a building ontology - a CityGML-based knowledge structure, planned to be extensible to specific architecture styles - through several guiding data structures such as structured XML and ontology-based grammar. Regarding the supporting process, a treemap approach is used to subdivide the building layout into floor plan areas. During the development of this work, several improvements were progressively made to the treemap in order to enable the subdivision of different constraint polygon types which range from rectangles to arbitrary shapes. Moreover, in the most mature work stage, a method concerning inner room walls adaptation is addressed. Next, a set of operations is performed, from the marking transitions step to the extrusion process that provides the 3D aspect. In addition, an experimental stochastic approach is proposed to automate the production of random buildings using this procedural modelling methodology.

A set of tests was made to demonstrate the capabilities of the proposed methodology 
in producing distinct building formats (buildings constrained by convex and nonconvex shapes, houses with specific room constraint walls) and different architectonic structures (general purpose houses, roman domus) in short time periods. Moreover, the effectiveness of the treemap approach in subdividing random layouts is shown, along with a generic stochastic process for automatic building generation and also some computational performance measurements.

Keywords: Procedural Modelling, Procedural Generation, Building Ontology, Building modelling, Ontological Grammar, Arbitrary Convex Shapes, Fake-concave, ComputerAided Design. 


\title{
Modelação Procedimental Baseada em Ontologia de Edifícios Atravessáveis Compostos por Formas Arbitrárias
}

\author{
Telmo Miguel Oliveira Adão \\ Tese submetida à Universidade de Trás-os-Montes e Alto Douro \\ em cumprimento parcial de requisitos \\ para a obtenção do grau de Doutor
}

Resumo - Modelos virtuais 3D de edifícios são usualmente utilizados em áreas como a arquitetura e videojogos para fins de visualização de projetos de casas e povoamento de cenários virtuais, respetivamente. Tradicionalmente, a produção destes modelos requer mão-de-obra altamente especializada e consideráveis quantidades de tempo. Para abordar esta questão, muitos investigadores desenvolveram técnicas semiautomáticas para produzir modelos virtuais de forma expedita. Estas técnicas procedimentais providenciam diferentes formas de geração de edifícios, incluindo interiores e fachadas exteriores, que servem vários propósitos (por exemplo, geração de conteúdo para videojogos ou reconstruções arqueológicas). No entanto, as técnicas existentes com foco na construção de interiores normalmente só suportam a geração de plantas restritas por formas regulares ou polígonos de contorno obtidos a partir de conjuntos de retângulos. Ao mesmo tempo, a possibilidade de modelar quartos interiores através da especificação das suas paredes de restrição permanece pouco explorada. Além disso, a maioria das soluções de geração procedimental existentes recorrem a gramáticas complexas referentes aos aspetos geométricos, ou então, estruturas semânticas desenvolvidas para projetos com requisitos específicos, desconsiderando os standards desenvolvidos para ambientes urbanos virtuais, concretamente, CityGML.

No sentido de abordar as questões indicadas, uma nova metodologia de modelação procedimental é proposta nesta tese, tendo como foco a produção de modelos virtuais de edifícios, incluindo exteriores circunscritos por formas arbitrárias e interiores formados por polígonos convexos. A regulação da metodologia é fornecida por uma ontologia para edifícios - que pode ser vista como um conjunto relacional de entidades baseadas em CityGML, extensíveis a estilos arquitetónicos específicos - através de várias estruturas de dados, tais como XML estruturado e gramática baseada na ontologia. Relativamente ao processo de suporte da repartição de espaço, uma abordagem treemap é usada para subdividir o layout representativo de uma dada base de edifício em subáreas inerentes a contentores e quartos interiores. Durante o desenvolvimento deste trabalho, diversas melhorias foram feitas ao treemap de forma progressiva, com o objetivo de permitir a subdivisão de diferentes tipos de polígonos de restrição que variam entre retângulos a formas arbitrárias. Além disso, na fase final deste trabalho, foi incorporado um método para a adaptação de paredes de quartos. Na sequência da subdivisão, vem um conjunto de operações que vai desde 
a marcação das transições até à extrusão das paredes que fornece o aspeto 3D. Também, uma abordagem estocástica experimental é proposta para automatizar a geração aleatória de edifícios, utilizando esta metodologia de modelação procedimental.

Um conjunto de testes foi feito para demonstrar as capacidades da metodologia proposta na produção de edifícios com formatos distintos (edifícios limitados por formas convexas e não-convexas e quartos com um número específico de paredes de restrição) e diferentes estruturas arquitetónicas (casas de propósito geral, domus romanas) em curtos períodos de tempo. Além disso, a eficácia da abordagem treemap na subdivisão de layouts é mostrada, juntamente com um processo estocástico experimental para a geração automática de edifícios e também algumas medições de desempenho computacional.

Palavras-chave: Modelação Procedimental, Geração Procedimental, Ontologia para Edifícios, Modelação de Edifícios, Gramática Baseada na Ontologia, Formas Convexas Arbitrárias, Falso-côncavo, Design Assistido por Computador. 


\section{Acknowledgments}

I want to use this opportunity to express my sincere gratitude to everyone that directly or indirectly participated on this important journey of my life, which is ending now.

Institutionally, I would like to thank to the Magnificent Rector of the University of Trás-os-Montes e Alto Douro, Professor António Augusto Fontainhas Fernandes and to his predecessor, Professor Carlos Alberto Sequeira, to the President of the School of Science and Technology, José Boaventura Cunha and to his predecessor Professor José Afonso Bulas Cruz by the facilities and means provided for the realization of this work.

A special thanks to my supervisors, Professor Luís Magalhães and Professor Emanuel Peres, by all of the support provided and useful interventions along this work. I am also very thankful to them by the vote of confidence and their friendship, which were crucial to the conclusion of this journey.

To all of my friends and colleagues, specially to Miguel Melo, my battle partner and loyal friend and also to his girlfriend, Indalécia Melim, for the good moments of leisure that we have spent together. To Simão Cardeal, Davide Borges, António 
Santos and Miguel Pereira, my trusted pals and unforgettable mates, to my dear graduation colleagues and friends, specially to Nuno Cunha, Nuno Liberato, Martinho Moreira, Carina Jerusa and Filipa Moura for the companionship and also to Martinho Gonçalves who partially shared this journey with me: my heartfelt thanks.

Another manifestation of gratitude must be addressed to the officials of the Engineering Department for their sympathy and availability to assist and support in situations related with documentation and classrooms: thank you for the laughs and thank you for your patience every time I lost the key of the research lab.

Thank you to INESC-Tec for supporting me in the beginning of this research process, specially to Ricardo Batista and Pedro Brandão, who provided me valuable tips during the development of this work.

I want to express my gratitude to my parents, brother and my family in general, for all the love, trust and support.

A very special thanks to my soulmate Dércia, for being such a good partner. Thank you for your love, friendship, support and remarkable patience, summarily, thank you for being the woman that I want to keep for life and beyond.

Finally, I would like to thank to those who have believed, for the vote of confidence and, to those who have doubted, for the challenge.

Thanks very much to all of you!

UTAD, Vila Real

Telmo Adão

$3^{\text {rd }}$ of December, 2015 


\section{General Index}

$\begin{array}{ll}\text { Abstract } & \text { xi }\end{array}$

$\begin{array}{ll}\text { Acknowledgments } & \text { XV }\end{array}$

Table Index $\quad$ xxi

$\begin{array}{ll}\text { Figure Index } & \text { xxiii }\end{array}$

Glossary, acronyms and abbreviations $\quad$ xxvii

1 Introduction $\quad 1$

1.1 Content Production for Virtual Environments . . . . . . . . . . . . . 2

1.2 Main Concepts . . . . . . . . . . . . . . . . . 4

1.2.1 Production of buildings through procedural generation . . . . 4

1.2.2 Regulation through ontologies . . . . . . . . . . . . 5

1.2.3 Ontology-based procedural modelling vs. Building Information Modelling . . . . . . . . . . . . . . 5 5

1.3 Motivation and Goals . . . . . . . . . . . . . . . . . 6

1.4 Main Contributions . . . . . . . . . . . . . . . . . . . 9

1.5 Assumptions . . . . . . . . . . . . . . . . . . . 10

1.6 Document Organization . . . . . . . . . . . . . . 11

2 Ontologies and Procedural Modelling $\quad 13$

2.1 Ontologies on Virtual Environments . . . . . . . . . . . . . . . . . . . 14 
2.1.1 Virtual representations based on ontologies . . . . . . . . . 15

2.1.2 CityGML: a 3D urban environment standard . . . . . . . . . 19

2.2 Procedural Modelling of Virtual Urban Environments . . . . . . . . . 22

2.2.1 L-systems for procedural modelling . . . . . . . . . . . . . 22

2.2.2 Detailing facades through split grammars . . . . . . . . . . . 23

2.2.3 Semi-automatic digital reconstruction of old buildings considering GIS data-based topology . . . . . . . . . . . . . . . . 25

2.2.4 Random extrusion of floors . . . . . . . . . . . . . . . . 25

2.2.5 Feature-based decomposition of facades . . . . . . . . . . 26

2.2.6 Computer generated architecture for buildings production . . 27

2.2.7 Building generation based on facade view acquisition . . . . . 30

2.2.8 Digital map-based generation of 3D buildings with multiple

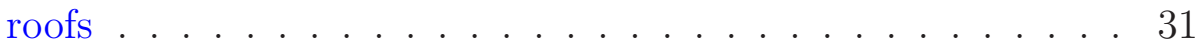

2.2.9 City Modelling Procedural Engine (CMPE) . . . . . . . . . . 31

2.2.10 Procedural generation 3D (PG3D) solution . . . . . . . . . . . 32

2.2.11 Ontology-based generation of urban environments and building exteriors . . . . . . . . . . . . . 33

2.3 Procedural Modelling of Virtual Traversable Buildings . . . . . . . . 35

2.3.1 LaHave House: an automated architectural design service . . . 35

2.3.2 Procedural generation of buildings using graphs and expansion algorithms . . . . . . . . . . . . . . . 35

2.3.3 Building indoors generation using constructive solid geometry algorithms . . . . . . . . . . . . . . . 37

2.3.4 Lazy production of virtual building interiors . . . . . . . . . . 37

2.3.5 Interior rooms generated through Voronoi diagrams and constrained by convex layouts . . . . . . . . . . . . . . . . 38

2.3.6 Rule-based generation/reconstruction of buildings . . . . . . . 39

2.3.7 Squarified treemaps for virtual buildings generation . . . . . . 40

2.3.8 Residential buildings generation based on bayesian networks . 41

2.3.9 Grid approach focusing floor plan generation . . . . . . . . . . 42

2.3.10 Generative Modelling Language (GML) for the production on virtual buildings . . . . . . . . . . . . . . . 43

2.3.11 Component-based modelling of virtual buildings . . . . . . . . 44

2.3.12 Producing virtual 3D buildings from pre-designed floor plans . 44

2.3.13 Ontology-based generation of traversable buildings . . . . . . 46

2.4 Summary . . . . . . . . . . . . . . . . . . . . . . . . . 48

3 Proposed Procedural Generation Methodology Overview 55

3.1 Problem Definition and General Proposed Framework . . . . . . . . . 55

3.2 Ontology for Buildings . . . . . . . . . . . . . . . . . . . 59 
3.2.1 Generic ontology . . . . . . . . . . . . . . . . . 59

3.2.2 Generic ontology elements . . . . . . . . . . . . . 60

3.2.3 Extending the generic ontology to the roman architecture . . . 62

3.2.4 Roman ontology elements . . . . . . . . . . . . . . 63

3.3 Procedural Modelling Methodology Overview . . . . . . . . . . . . 65

3.3.1 Building Floor plan definition . . . . . . . . . . . . 65

3.3.2 Extrusion, roofing and completions .......... 66

3.4 Summary . . . . . . . . . . . . . . . . . . 66

4 Generation of Virtual Buildings Formed by Rectangles $\quad 69$

4.1 Preliminary Procedural Modelling Methodology Proposal . . . . . . . 70

4.1 .1 Ontology integration . . . . . . . . . . . . . . 71

4.1.2 Methodology regulation: definition rules . . . . . . . . . . 72

4.1.3 Methodology regulation: restriction rules . . . . . . . . . . 76

4.1.4 Procedural modelling approach . . . . . . . . . . . . . . . 77

4.2 Implementation Prototype . . . . . . . . . . . . . . . . . . . . . 83

4.3 Preliminary Tests and Results . . . . . . . . . . . . . . . . . . . 85

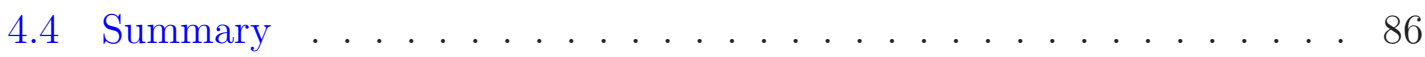

5 Generation of Virtual Buildings Constrained by Convex Shapes 89

5.1 Enhanced Procedural Modelling Methodology Proposal . . . . . . . . 90

5.1.1 Ontology-based data model to guide definition rules . . . . . . 91

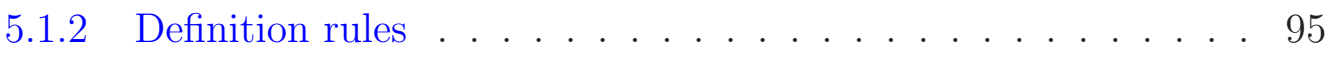

5.1 .3 Restriction rules . . . . . . . . . . . . . . 96

5.1.4 Moderation process for definition rules . . . . . . . . . . . 98

5.1.5 Procedural modelling generation process . . . . . . . . . . . 99

5.2 Enhanced Methodology System Implementation . . . . . . . . . . . . 106

5.2.1 An ontology-based XML for virtual buildings definition (XML4BD) 107

5.2 .2 Rules moderator module . . . . . . . . . . . . . . 110

5.2.3 Procedural modelling module . . . . . . . . . . . . . . . . . 112

5.3 Preliminary Tests and Results . . . . . . . . . . . . . . . . . . 113

5.4 Summary . . . . . . . . . . . . . . . . . . 116

6 Generation of Virtual Buildings Composed by Arbitrary Shapes 119

6.1 Final Procedural Modelling Methodology Proposal . . . . . . . . . . . 121

6.1.1 Ontology-based Grammar . . . . . . . . . . . . . . . 123

6.1.2 Moderating ontology-based grammar rules . . . . . . . . . . 126

6.1.3 Procedural modelling generation process . . . . . . . . . . . 127

6.1.4 Stochastic rules selection for the automatic generation of virtual buildings . . . . . . . . . . . . . . . . . 134 
6.2 Final Procedural Modelling Methodology Implementation . . . . . . . 138

6.2.1 Deterministic Tool . . . . . . . . . . . . . . . . . . 140

6.2.2 Stochastic tool . . . . . . . . . . . . . . . . 141

6.3 Preliminary Tests and Results . . . . . . . . . . . . . . . . . . . 144

6.4 Summary . . . . . . . . . . . . . . . . . . . 146

7 Procedural Modelling Methodology Evaluation $\quad 149$

7.1 Virtual Buildings Constrained by Arbitrary Shapes . . . . . . . . . . 150

7.1.1 Simple building model . . . . . . . . . . . . . . . . . . 151

7.1.2 U-shaped building model . . . . . . . . . . . . . . . . . 152

7.1.3 L-shaped building model . . . . . . . . . . . . . . . . . 153

7.1.4 Arbitrary building model with three connected corridors . . . 153

7.2 Interior Divisions' Walls Adaptation . . . . . . . . . . . . . . . . . 155

7.2.1 L-shaped building model (six divisions) . . . . . . . . . . . . 155

7.2.2 L-shaped building model (nine divisions) . . . . . . . . . . . . 155

7.2.3 T-shaped building model . . . . . . . . . . . . . . . . 157

7.2.4 Building model with a complex layout . . . . . . . . . . . . 157

7.3 Subdivision Provided by the Treemap Approach . . . . . . . . . . . 158

7.4 Generating Structures Stochastically . . . . . . . . . . . . . . . 159

7.5 Ontology-based Architectural Derivation . . . . . . . . . . . . . . 160

7.5.1 Generic building and roman domus overview . . . . . . . . . . 162

7.5.2 Structural extensions and differences . . . . . . . . . . . 163

7.6 Performance Results . . . . . . . . . . . . . . . . . . . . 163

7.7 Summary . . . . . . . . . . . . . . . . . . . . 168

8 Conclusions $\quad 171$

8.1 Thesis Summary . . . . . . . . . . . . . . . . 171

8.1.1 Building Ontology . . . . . . . . . . . . . 172

8.1.2 Procedural Modelling Methodology . . . . . . . . . . . . 172

8.2 Discussion and Future Work . . . . . . . . . . . . . . . . . . . 174

8.3 Final Remarks . . . . . . . . . . . . . . . . . . . . 177

$\begin{array}{ll}\text { References } & 179\end{array}$ 


\section{Table Index}

2.1 Summary of the procedural modelling works, regarding buildings with interiors. . . . . . . . . . . . . . . . . . . 49

4.1 Roman domus divisions classified by functional zones. . . . . . . . . . 73

5.1 Reconstructed roman buildings of Conimbriga (Portugal) . . . . . . . 113

5.2 Ficticious virtual building models representing ancient structures and produced by the system that implements the methodology for generating buildings constrained by convex shapes. . . . . . . . . . . . . . . 115 



\section{Figure Index}

1.1 Examples of real-world buildings floor plans composed by inner and outer non-rectangular shapes. . . . . . . . . . . . . 8

2.1 A UML diagram representing the top classes of the CityGML standard (Kolbe, 2009) . . . . . . . . . . . . . . . . . . . . . . . 20

2.2 UML diagram showing a simplified excerpt from the CityGML building model (Kolbe, 2009). . . . . . . . . . . . . . . . . . . 21

2.3 L-System operation: an iterative replacement system using alphabets. 23

2.4 Set of operations performed by a split grammar (Wonka et al., 2003). 24

2.5 Process of floor extrusion proposed by Greuter et al. (2003). . . . . 26

2.6 Merging of volumetric shapes resulting from CGA grammar rules. . . 28

2.7 Virtual Rome produced by the system of Dylla et al. (2009), using manual and procedural models. . . . . . . . . . . . . . . . 29

2.8 Boavista roundabout produced by PG3D (Silva and Coelho, 2011). . 33

2.9 An house with interiors produced by Martin's approach (Martin, 2006). 36

2.10 Building with floors produced in real-time through the lazy approach proposed by Hahn et al. (2006). . . . . . . . . . . . . . . . 38

2.11 Example of a building structure generated by Rinde (2008). . . . . . 39

2.12 Virtual environment depicting roman houses from Rodrigues (2010). . 40 
2.13 Squarified treemaps operation (Bruls et al., 1999). . . . . . . . . . . . 41

2.14 The process of house generation based on bayesian networks, trained with real data (Merrell et al., 2010). . . . . . . . . . . . . . . . . 42

2.15 Virtual environments produced using the tool developed by Santos et al. (2011). . . . . . . . . . . . . . . . . . 46

2.16 Virtual World generator process proposed by Trescak et al. (2010) and exemplified through an auction system. . . . . . . . . . 48

3.1 Generic buildings' ontology: a semantic abstract schema that defines the connections between the elements that can be part of a building. 61

3.2 The WorldObject parent and the inheritance mechanism . . . . . . . 61

3.3 The specialization of the generic building ontology into roman architectural objects. . . . . . . . . . . . . . . . . . 6 . 63

4.1 Representative schema of the connections possibilities between functional zones. . . . . . . . . . . . . . . . . . . . . . . 76

4.2 Floor plan generation, using a treemap approach for dividing a roman domus into rectangular divisions. . . . . . . . . . . . . . . . . 78

4.3 Procedural modelling approach main stages, from the treemap-based floor plan division to the wall extrusion. . . . . . . . . . . . . 79

4.4 Generic work-flow diagram depicting the behaviour of the treemap subdivision process. . . . . . . . . . . . . . . . . 81

4.5 Generic work-flow diagram depicting the behaviour of the transition placement process. . . . . . . . . . . . . . . . . . . . . 82

4.6 Generic workflow diagram depicting the behaviour of the extrusion process, for a single AbstractBuildingElement. . . . . . . . . . . . . . 84

4.7 Final layout of a generated domus including interiors and a exterior. . 87

5.1 Simplified ontology-based data model. . . . . . . . . . . . . . 93

5.2 Workflow depicting the process of balancing division weights through space borrowing. . . . . . . . . . . . . . . . . . . . 100 
5.3 General workflow depicting the adjustments made to a given building element during the floor plan division process of a virtual building constrained by a convex shape (valid for building parts, divisions and temporary containers). . . . . . . . . . . . . . . . . . . 104

5.4 Example of a treemap structure defining a floor plan arrangement, and respective floor plan subdivision process. . . . . . . . . . . 105

5.5 Architecture of the system that implements the methodology for generating virtual buildings constrained by arbitrary shapes, considering XML4BD specifications as input rules. . . . . . . . . . . . . . 106

5.6 Example of a XML4BD node system and resulting 3D virtual model . 108

6.1 Example of the fake-concave technique behaviour. . . . . . . . . . . 128

6.2 Workflow depicting the addition of division wall segments. . . . . . . 130

6.3 Workflow depicting the operations regarding the deletion of a division's wall segments. . . . . . . . . . . . . . . . . . . . 131

6.4 Geometric operations to add or remove wall segments for division's shape adaptation. . . . . . . . . . . . . . . . . . . 132

6.5 Stochastic process regarding the generation of random virtual buildings. 135

6.6 Simplified scheme of the stochastic process, depicting the main steps regarding the random selection and arrangement of both priority-one and priority-two divisions. . . . . . . . . . . . . . . . 137

6.7 Screenshot of the procedural modelling system implementing the stochastic and parametric tools. . . . . . . . . . . . . . . . . . . . . 139

6.8 Software toolkit backoffice screenshots excerpts regarding texture and restriction parameters. . . . . . . . . . . . . . . . . 140

6.9 Set of operations that a user can perform in the deterministic tool GUI.142

6.10 Preliminary results depicting the traversable buildings generated with the final methodology. . . . . . . . . . . . . . . . . . . . . . 145

6.11 Preliminary results of the stochastic approach. . . . . . . . . . . 145

7.1 Simple general purpose building (based on the generic ontology) . . . 152

7.2 U-shaped general purpose building (based on the generic ontology). . 153

7.3 L-shaped general purpose building (based on the generic ontology). . 154 
7.4 A general purpose building (based on the generic ontology) with three corridors connected through doorless transitions. . . . . . . . . . . 154

7.5 L-shaped building demonstrating inner walls adaptation . . . . . . . 156

7.6 Second L-shaped building that demonstrates inner walls adaptation . 156

7.7 T-shaped building to demonstrate inner walls adaptation. . . . . . . . 157

7.8 Fourth virtual building model for the demonstration of inner walls adaptation. . . . . . . . . . . . . . . . . . . . . . 158

7.9 Floor plan subdivision examples with four distinct structures . . . . . 159

7.10 Stochastic production of virtual buildings obtained from three fixed sets of divisions and a fourth set with randomly selected divisions. . . 161

7.11 Ontology-based generation of a generic building and a roman domus . 162

7.12 Structural differences between the domus and the generic building . . 164

7.13 Chart depicting the performance tests made to five groups of virtual buildings with a gradual increment of building parts per group and divisions per generation. . . . . . . . . . . . . . 165

7.14 Results of the performance tests made to measure the computational resources spent by the application of the procedural modelling methodology in the generation of virtual buildings characterized by internal walls adaptation, presented in section $7.2 \ldots \ldots$. . . . . . . . 166

7.15 Results of the performance tests made to the structures subdivision process presented in section 7.3 . . . . . . . . . . . . . . . 167

7.16 Results of the performance tests made to the generation of ontological structures presented in section 7.5 . . . . . . . . . . . . . . . 168 


\section{Glossary, acronyms and abbreviations}

\section{Acronyms list}

$\begin{array}{ll}\text { Acronym } & \text { Expansion } \\ 2 \mathrm{D} & \text { Two Dimensional } \\ \text { 3D } & \text { Three Dimensional } \\ \text { ASCII } & \text { American Standard Code for Information Interchange } \\ \text { BAW } & \text { Building with Adapted Walls } \\ \text { BIM } & \text { Building Information Modeling } \\ \text { BMG } & \text { Building Model Generator } \\ \text { BSP } & \text { Binary Space Partitioning } \\ \text { CAD } & \text { Computer-Aided Design } \\ \text { CD } & \text { Cyber Devices } \\ \text { CGA } & \text { Computer Generated Architecture } \\ \text { CityGML } & \text { City Geography Markup Language } \\ \text { CMPE } & \text { City Modeling Procedural Engine } \\ \text { CPU } & \text { Central Processing Unit } \\ \text { CSG } & \text { Constructive Solid Geometry } \\ & \end{array}$




\section{Acronym}

DS

ERAS

Fpms

GB

$\mathrm{GHz}$

GIS

GML

GTA

GUI

HarvANA

IFC

LRU

L-System

MRF

$\mathrm{MB}$

MMORPG

$\mathrm{ms}$

NFS

OCG

OCR

$\mathrm{PC}$

PG3D

RAM

$\mathrm{r} / \mathrm{w}$

SEMIC

UML

VFDM

\section{Expansion}

Division Structures

Expedite virtual Reconstruction of Cultural HeritAge Sites

Floor planned modules

Gigabyte

Gigahertz

Geographic Information System

Generative Modeling Language

Grand Theft Auto

Graphical User Interface

Harvesting and Aggregating Networked Annotations

Industry Foundation Classes

Last Recent Use

Lindenmayer System

Markov Random Field

Megabyte

Massive Multiplayer Online Role-Playing Game

millisecond

Need For Speed

Open Geospatial Consortium

Optical Character Recognition

Personal Computer

Procedural Generation 3D

Random Access Memory

$\mathrm{read} /$ write

Semantic Modeling of Interactive 3D Content

Unified Modeling Language

Virtual Factory Data Model 


$\begin{array}{ll}\text { Acronym } & \text { Expansion } \\ \text { VFM } & \text { Virtual Factory Manager } \\ \text { VirCA } & \text { Virtual Collaboration Arena } \\ \text { VRML } & \text { Virtual Reality Modeling Language } \\ \text { VW } & \text { Virtual World } \\ \text { X3D } & \text { Extensible Three-Dimensional } \\ \text { XML } & \text { Extensible Markup Language } \\ \text { XML4BD } & \text { Extensible Markup Language for Building Definition }\end{array}$

\section{Abbreviations List}

$\begin{array}{ll}\text { Abbreviation } & \text { Meaning(s) } \\ \text { e.g. } & \text { from latin exempli gratia; for example } \\ \text { et al. } & \text { from latin et alii; and others (co-authors) } \\ \text { i.e. } & \text { from latin id est; that is }\end{array}$





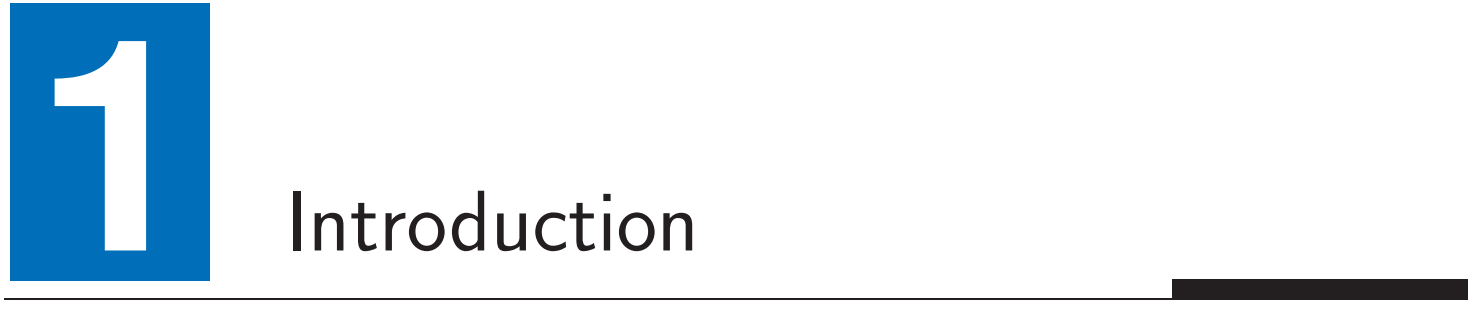

Research and business areas like architecture, archaeology or videogames require the use of virtual structures, specifically buildings, to satisfy different requirements such as: the presentation of building projects, testing of hypothesis based on virtual models that enable theory formulations about missing or severely damaged ancient buildings or, on the other hand, the population of urban environments in an extensive way. Commonly, the production of these models requires highly skilled manpower and a considerable amount of time. Alternatively, procedural modelling proposes a semi-automatic, cost-effective and faster way of providing accurate virtual buildings representations.

During the last two decades, the scientific community researching procedural modelling has explored different methodologies to solve the generation of urban environments and building interiors with interesting results. However, some issues remain unexplored. For example, methodologies for producing 3D buildings constrained by arbitrary shapes are still scarce, as until now, most of the existing solutions focus the generation of floor plans composed by squared or regular shapes. On the other hand, the semi-automatic modelling of interior rooms with non-regular formats is rarely addressed. 
To overcome these issues, a new procedural modelling methodology capable of producing 3D building models externally constrained by arbitrary shapes and having internal divisions delimited by convex shapes is proposed. It is regulated through a building ontology that was created considering CityGML (Gröger et al., 2008, 2012) as basis: a standard proposed by Open Geospatial Consortium that details virtual urban environments. A set of instructions provided by ontology-based rules guides the proposed methodology in the following sequence: a constraint polygon is split into inner rooms; the transitions are placed between the resulting rooms; the walls extrusions are applied; finally, the placement of the roofs, rooms ceilings and floors takes place.

This introductory chapter will expatiate around the production of contents for virtual environments, procedural modelling and also ontologies. Motivation and goals for this work are then presented along with some assumptions. This chapter ends with the main contributions and orientations regarding document organization.

\subsection{Content Production for Virtual Environments}

Content production for virtual environments is an important subject as it is directly related with parameters such as production cost and development time, which have a significant impact in how well a business or research performs. The conventional production of these models - specifically, using manual modelling - requires highly skilled manpower and a considerable amount of time to achieve the desired virtual contents, in a process composed by many stages that are typically repeated over time.

As technology continues to evolve at a faster rate - more processing power, faster and larger memory, increased disk space at better $\mathrm{r} / \mathrm{w}$ rates and more powerful graphic boards - new paradigms are emerging to provide more efficient and cost-effective solutions for business that depend on virtual contents. Among them is procedural modelling which can be seen as an assortment of techniques that aim to automatize the production of virtual models through the assimilation of patterns and 
algorithmic approaches that assume the role of content production engines. From the perspective of some business and research areas (e.g. architecture, archaeology, videogames producers), the overwhelming use of resources can now be drastically reduced, leading to the increase of competitiveness. Moreover, man-skilled labour can now be concentrated in the validation and improvement of the automatically produced models by adding or altering particularities and details that might make them closer to the expected results, considering the requirements of a given modelling task in a certain context.

The application fields are numerous. Videogames industry is perhaps one of the most obvious cases, due to the use of complex road networks and rich urban environments, pretty noticeable in games such as Grand Theft Auto ${ }^{1}$ or Need For Speed ${ }^{2}$. Actually, NFS was a case study for Watson et al. (2008) who had underlined the applicability and importance of procedural modelling in the production of certain game contents such as buildings and road networks, due to its cost-effective and dynamic nature. Moreover, they suggest that designers who demand for automatic ways of generating game contents to avoid tedious and repetitive hand-made tasks, can be supported by procedural modelling tools to generate the first set of urban objects, that afterwards can be customized to make them look like what they have projected.

The same modelling style can be used in the archaeological research area, even in damaged structures - as it is pointed out by Müller et al. (2006a), Rodrigues et al. (2008b) or Dylla et al. (2010) - to aid, for example, in the proposal of hypothesis that can be valuable for the formulation of theories among that scientific community.

Another application field is 3D cinema. Enterprises like Pixar ${ }^{3}$ or Dreamworks Animation $^{4}$ are specialized in producing $3 \mathrm{D}$ movies that include human and animal

\footnotetext{
${ }^{1}$ Grand Theft Auto, also known as GTA, is a well-know role playing game series, developed by Rockstar. For more information, check the link http://www.rockstargames.com/ grandtheftauto/.

${ }^{2}$ Need For Speed or NFS, is a racing game series developed by Electronic Arts. For more information, check the link http://www. needforspeed.com/.

${ }^{3}$ Pixar is a digital animation enterprise that belongs to the Walt Disney Company. For more information, check the link http://www.pixar.com/.

${ }^{4}$ Dreamworks Animation is a north-american studio specialized in animation movies. For more information, check the link http://www.dreamworksanimation.com/.
} 
characters, cities, villages and forests. Some of their productions already take advantage from procedural modelling techniques. For example, in the Monters Inc. (Pixar) movie, the hair of Sulley character is procedurally animated (Cohen, 2001). Another example is the fracturing and debris procedural technique that was developed for Kung-Fu Panda (Dreamworks) in order to be applied in several scenes involving massive destruction of structures (Lee et al., 2008b).

These were just a few examples intending to show procedural modelling versatility. However, others will be provided in the next chapters, to demonstrate the wide range of applicability of this modelling style in the generation of structures - specifically, buildings - expeditiously and demanding low user interaction.

\subsection{Main Concepts}

The concepts inherent to the development of this work will be presented, namely, ontologies and procedural modelling. Building Information Modelling (BIM) disambiguation closes the section.

\subsubsection{Production of buildings through procedural genera- tion}

The production of buildings and urban environments are major concerns for the procedural modelling area. Many works (Parish and Müller, 2001; Müller et al., 2006b; Dylla et al., 2010; Silva and Coelho, 2011) present different approaches for the procedural generation of extensive urban environments, considering the exterior facades. These solutions have demonstrated to be a reliable alternative to manual approaches, since they are also capable of producing representations endowed with high levels of detail and visual accuracy. Regarding time consumption, the procedural solutions are incomparably faster. The same conclusions are valid for the generation of buildings considering their interiors (Rodrigues et al., 2008b; Marson and Musse, 2010; Tutenel et al., 2011; Merrell et al., 2010) which proposes the fully 
production of such structures including exterior facades, inner rooms and also the transitions that ensure transitivity among them.

\subsubsection{Regulation through ontologies}

Ontologies are knowledge structures capable of describing a system, namely the relations between its parts. They have been successfully applied in different solutions that require the use of virtual models/environments (Lee et al., 2008a; Attene et al., 2009; Hunter and Gerber, 2010) to achieve a wide variety of purposes that range from the planning of neurosurgery operation to the cataloguing of museum artefacts. A few procedural modelling solutions also used them to guide the process of generating virtual models (Liu et al., 2008; Trescak et al., 2010; Tutenel et al., 2011). The results are interesting. However, most of these procedural modelling solutions seem confined to the context for which they were developed, disregarding standards oriented for virtual environments.

\subsubsection{Ontology-based procedural modelling vs. Building In- formation Modelling}

This subsection intends to clarify the main differences between ontology-based procedural modelling approach and Building Information Modelling (BIM) which, due to the common use of semantics and similar goals, are liable to cause confusion.

BIM supports the development and use of a computer generated model to simulate the different stages of a facility such as planning design and construction. Its preciseness, flexibility and huge range of possibilities make it suitable for construction professionals (Azhar, 2011). It is a complex standard that mixes semantic and geometry and contains a complete set of information - including, for example, the air conditioning system, the building structure or even the materials of its walls and which requires expertise and labor when dealing with it, in order to meet client requirements and also legal and physical rules. 
On the other hand, ontology-based procedural modelling is concerned with the rapidly and faithful visualization of virtual structures, disregarding imperceptible details - such as the skeleton structure of a building - which can be harmful to the fluidity that is required, for example, in a video-game. During the exploration of a virtual scenario, these details are usually not needed for observation. Thereby, the performance issues are avoided at two levels: in the structures generation for further visualization; and in the virtual environment navigation itself that is only provided with required contents for visualization, possibly using a level-of-detail (LOD) loading strategy for computational resources management purposes.

Regarding the requirements of this procedural modelling work, a CityGML-based ontology was adopted and consists in a semantic structure simpler than BIM semantics, as it will be detailed in later sections. This option does not reject the possibility of having the resulting models integrated in BIM-based projects, since one of the CityGML (Gröger et al., 2008, 2012) concerns is to improve the interoperability between the referred construction standard and urban virtual environments (Kolbe, 2012).

\subsection{Motivation and Goals}

Real-world buildings are made up of very diverse geometries. Probably, the most commonly found sustaining floor plans are based on rectangular shapes. However, other contemporaneous and historical buildings are composed by wider sets of geometries. Some examples are provided on Figure 1.1. They include the floor plans of the following randomly selected buildings: Centre Pompidou-Metz ${ }^{5}$, Metz, France;

\footnotetext{
${ }^{5}$ Centre Pompidou-Metz, situated in Metz, France; retrieved from http://estelalopezrocha.blogspot.com, in 2014.
} 
Nantes' Paloma music complex ${ }^{6}$, France; Casa da Música ${ }^{7}$ situated in Porto, Portugal; the 39th floor of the Shard London Bridge ${ }^{8}$, London, United Kingdom; the 89th floor penthouse belonging to a Trump Tower ${ }^{9}$ located in Illinois, United States; SZA residential and business center ${ }^{10}$ located in Zagreb, Croatia; also historic buildings such as Cantaber and Insula of the Phallus Vase ${ }^{11}$, located in Conimbriga, Portugal; and finally, Doune Castle ${ }^{12}$ from Doune Village, Scotland.

Currently, procedural modelling methodologies that deal with traversable buildings, composed by non-square based polygons - as the ones that were presented in Figure 1.1 - including interiors (i.e. delimiting room walls) and exteriors (i.e. constraint building limits) are scarce. Moreover, notwithstanding the available and complete procedural modelling methodologies, some issues were identified:

- currently, the generation of virtual buildings with interior divisions composed by a customized number of constraint walls is poorly explored;

- most of the approaches generating virtual buildings only operate with constraint polygons based on rectangles;

- the absence of a semantic organization into a comprehensive and extensive ontology (or similar) based on standards - which is noticed in most of the procedural modelling solutions - might difficult the derivation of new architectonic styles and the exchange of information among heterogeneous systems regarding virtual environments, in general.

\footnotetext{
${ }^{6}$ Paloma music complex, situated in Nantes, France; retrieved from http://www. behance.net, in 2014.

${ }^{7}$ Casa da Música, situated in Porto, Portugal; retrieved from http://estelalopezrocha.blogspot.com, in 2014.

${ }^{8} 39$ th floor of the Shard London Bridge, situated in London, England; retrieved from http: //www. theplan.it, in 2014.

${ }^{9} 89$ th floor penthouse of the Trump Tower, situated in Illinois, United States; retrieved from http://chicago.curbed.com, in 2014.

${ }^{10} \mathrm{SZA}$ residential and business center, situated in Zagreb, Croatia; retrieved from http://www. designboom.com, in 2014.

${ }^{11}$ House of Cantaber and Insula of the Phallus vase ruins, situated in Conimbriga, Portugal; retrieved from http: //www. conimbriga.pt, in 2014.

${ }^{12}$ Doune Castle, situated in Doune Village, Scotland; retrieved from http://lostdelights.wordpress.com, in 2014.
} 

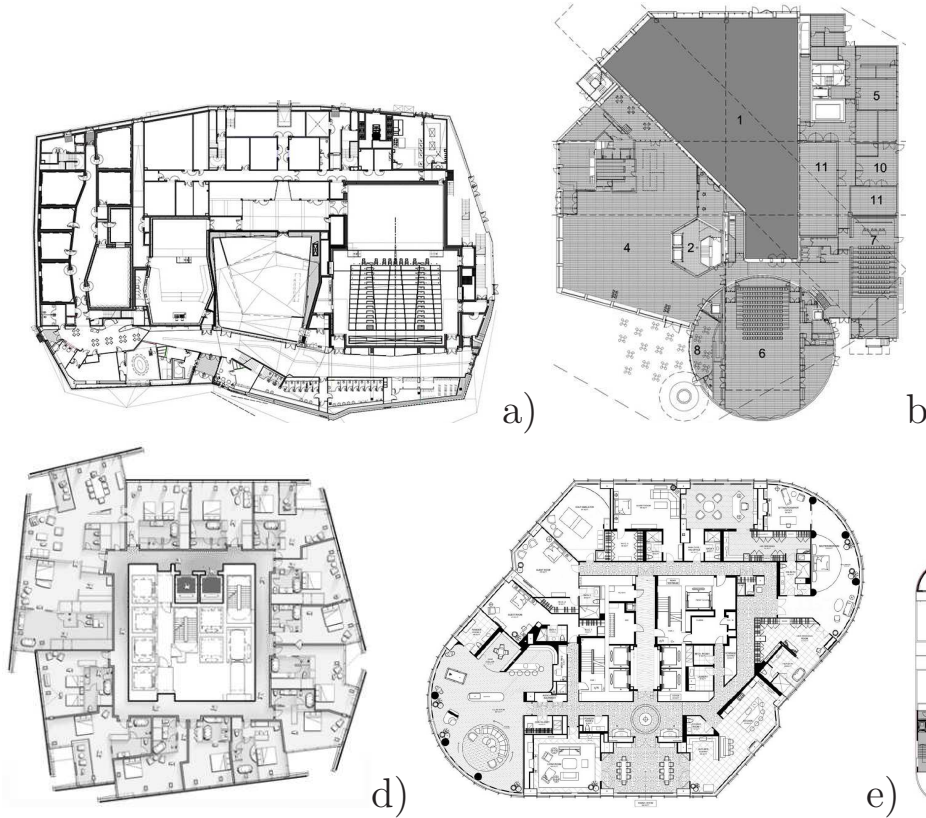

d)

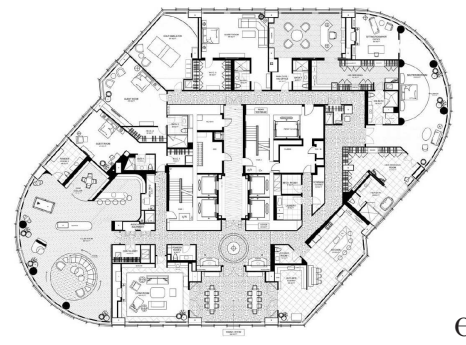

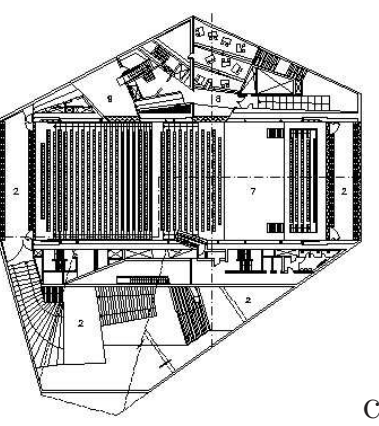

c)
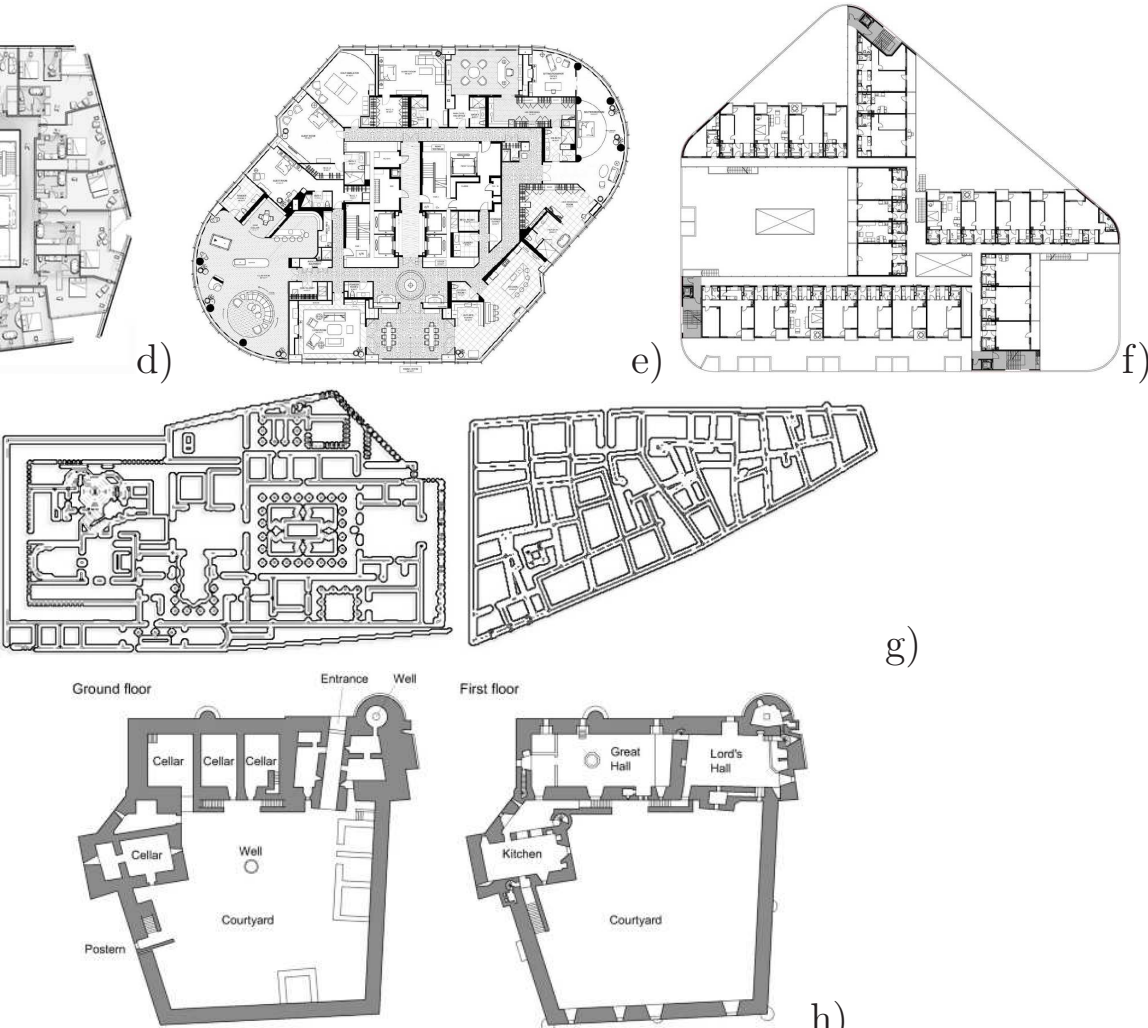

g)

h)

Figure 1.1 - Examples of real-world buildings floor plans composed by inner and outer nonrectangular shapes: a) Paloma music complex - Nantes, France; b) Centre Pompidou-Metz Metz, France; c) Casa da Música - Porto, Portugal; d) 39th floor of the Shard London Bridge - London, England; e) 89th floor penthouse of the Trump Tower - Illinois, United States; f) SZA residential and business center - Zagreb, Croatia; g) House of Cantaber (left) and Insula of the Phallus vase (right) ruins - Conimbriga, Portugal; h) Doune Castle - Doune Village, Scotland;

Thus, considering the aforementioned issues, the main goal of this thesis is to propose a methodology capable of producing traversable buildings internally and externally 
described by more shapes than the rectangular-based ones. Moreover, in order to guide the referred methodology through the modelling of multiple structures belonging to different architectonic styles (e.g. ancient roman style, neoclassic, postmodern), a flexible and extensible ontology is also proposed. It relies in CityGML (Gröger et al., 2008, 2012) which is an extensive and mature standard for virtual urban environments that documents a set of data models, including the generic composition and organization of the building entity.

Summing up, to fulfill the requirements of this work, the following list of specific goals need to be achieved:

- Identify the limitations that currently affect the procedural modelling research area;

- Propose of a novel methodology, capable of overcoming the identified issues;

- Develop a prototype to demonstrate and evaluate the proposed methodology;

- Carry out a performance evaluation;

- Discuss and compare the obtained results to other relevant works.

\subsection{Main Contributions}

This thesis main contributions focus on the proposal of a novel procedural modelling methodology supported by an extensible ontology. The following topics intend to identify the contributions:

- Development of a new procedural modelling methodology capable of producing traversable buildings constrained by arbitrary convex shapes, based on a pure treemap approach;

- Establishment of a process to change the format of the interior rooms, through wall number modification; 
- Adaptation of a "fake-concave" technique to support non-convex buildings layouts;

- Proposal of an extensible building ontology to guide the methodology process and support the generation of other architectural style buildings (e.g. roman houses);

- Presentation of some ontology-based structures - eXtensible Markup Language (XML) and ontological grammar - to provide the procedural modelling methodology with production rules;

- Suggestion of computer-managed processes for the stochastic generation of buildings;

- Development of a toolkit implementing the above-mentioned procedural modelling methodology and computer-managed processes for automating building production.

\subsection{Assumptions}

This section will present a set of topics that the reader must take into consideration, when consulting this thesis. They will help to clarify which aspects are addressed and which are not:

- The proposed methodology will only focus on the generation of buildings with one floor, despite the support of the ontology to achieve more than that;

- The proposal aims the generation of individual buildings that can possibly be integrated in more extensive urban environments;

- The proposed procedural modelling approach intends to be an alternative for the existing ones and aims to produce a wider range of structures described by an extensive range of geometries, rather than to be concerned with faithful architectonic representations; 
- Most of the generated models follow a deterministic approach. However, a stochastic alternative for the fully automated productions of buildings is proposed, constituting a preliminary approach devoid of real architectonic regulation;

- The proposed work does not address slanted walls;

- When discussing the proposed procedural modelling methodology, the term "room" is sometimes applied as having the same meaning as "division", which is formally characterized in the proposed ontology (addressed on chapter 3);

- All of the implementation versions regarding the proposed procedural modelling methodology were made under Microsoft's .NET framework ${ }^{13}$, using C\# programming language ${ }^{14}$ and also the XNA framework ${ }^{15}$;

- This thesis focus the procedural modelling of buildings, not the rendering aspects. To visualize the created models, a XNA-based previewer was developed and a Blender software ${ }^{16}$ was used.

\subsection{Document Organization}

Besides the introduction, this thesis is constituted by seven other chapters.

Chapter 2 addresses ontologies and its effective applications on several fields requiring virtual models and environments. Also, an extensive revision targeting the state-of-art on procedural modelling is presented and its main contents include urban environments, traversable buildings and ontological approaches.

\footnotetext{
${ }^{13}$ Microsoft .NET framework refers to a collection of programming libraries that enables the use of .NET platform functionalities (link: http://www.microsoft.com/net).

${ }^{14} \mathrm{C} \#$ is a powerful and flexible object-oriented programming language developed by Microsoft. More informations are available at the link https://msdn.microsoft.com/en-us/library/ $\mathrm{kx} 37 \times 362$. aspx.

${ }^{15}$ Microsoft XNA (not acronymed) framework is a software development kit for game production dedicated to Microsoft compatible devices (PC, Xbox). For more information, consult the following link: http://msdn.microsoft.com/xna.

${ }^{16}$ Blender is a free and professional computer aided design (CAD) software developed, maintained and distributed by Blender Foundation. It is used for 3D modelling, UV wrapping, texturing, raster graphics editing and others (link: http://www.blender.org).
} 
The third chapter (3) presents an overview of the proposed procedural modelling methodology for generating building's outlined and internally composed by arbitrary shapes. It includes the generic ontology, the derivation to the roman ontology and also the procedural modelling process, which succinctly explains the involved generation steps, from the floor plan subdivision to the walls extrusions.

Chapters 4, 5 and 6 will expose in detail each development and implementation stage of the proposed methodology. Chapter 4 addresses a first approach, which joins ontology and treemaps to achieve the creation of roman houses - uniquely composed by square shapes.

Chapter 5 presents the first traversable buildings constrained by convex shapes. A set of descriptions of historic roman buildings are converted to XML definitions based on ontology - and then, the reconstructions takes place using the procedural modelling methodology. The adaptation of this XML to a more adequate format, XML for buildings definition (XML4BD), is also documented along with the fictitious buildings that were produced as demonstration, in this development stage.

A substantial part of this work is revealed on chapter 6 . The proposed methodology, which allows the generation of buildings apparently constrained by non-convex shapes using the false-concave technique is explained. Here, the adaptations made to this approach that enable the modelling of the internal rooms through wall number definition are presented. Also, the new input rule set, which evolved to an ontologybased grammar and a stochastic approach, are addressed. This chapter ends with the presentation of a toolkit composed by a deterministic parametric tool and also a stochastic tool, that implements the complete procedural modelling methodology.

The methodology evaluation is addressed in the seventh chapter (7), using the aforementioned toolkit. A set of tests is exposed to evaluate the methodology in the following aspects: generation of simple structures, adaptation of inner walls to define the room shapes, treemap subdivision and architectural derivation capabilities. The stochastic approach is also evaluated. Finally, some performance tests are presented.

The final chapter - chapter 8 - ends this document by exposing the conclusions, a brief discussion and some final remarks. 


\section{Ontologies and Procedural Modelling}

Procedural modelling consists in a collection of semi-automatic processes and techniques applied to generate virtual environments and structures with a reduced demand for user interaction. These techniques have been studied, developed and applied by several researchers who recognize the potential of procedural modelling in reducing development time in projects requiring massive production of virtual structures such as traversable buildings or extensive urban environments. During the last few decades, several works were developed, addressing the generation of building exteriors uniquely composed by outer facades (e.g. Parish and Müller (2001), Wonka et al. (2003), Müller et al. (2006b), Carrozzino et al. (2009), Silva and Coelho (2011)) and traversable buildings with interior divisions (e.g. Hahn et al. (2006); Marson and Musse (2010); Merrell et al. (2010)). A subset of these works also relies on ontologies and semantics (e.g. Trescak et al. (2010), Liu et al. (2006), Liu et al. (2008)), which are knowledge organization based approaches that contributed, along with procedural modelling, to the methodology proposed in this document. Thus, the following subsections will expose a literature review regarding both matters. 


\subsection{Ontologies on Virtual Environments}

Over the years, several authors have defined and characterized "ontology" (e.g. Borgo et al. (1997), Noy and Hafner (1997), Benjamins and Fensel (1998)), while others were concerned with its applications in fields such as information systems and engineering (Wand and Weber (1990), Weber and Lybrand (1997), van Heijst et al. (1997)). All of them inspired Guarino (1998) and Chandrasekaran et al. (1999) in the formulation of their own concepts about these knowledge organization structures.

According to Guarino (1998), an ontology aims to describe a certain entity using a particular system of categories. In some areas like engineering - such as Artificial Intelligence - an ontology is established through a set of concepts and respective meanings (i.e. vocabulary) with a relation structure that intends to characterize a certain reality. The author also refers the increasing importance of ontologies for computer science, focusing in the information systems area, in which ontologies can play an important role due to their straight relation with components such as databases and program objects and application programs.

Chandrasekaran et al. (1999), shares a similar vision. The author states that this kind of structures intends to represent a set of facts related with a particular domain through the organization of the integrating knowledge concepts. This organization arises from the analysis over the domain fields, in which the following authors' assumption should be considered:

"Weak analyses lead to incoherent knowledge bases."

- Chandrasekaran et al. (1999)

One of the most interesting features of ontologies is the possibility of sharing knowledge. This promotes a re-usability and standardization. For example, a building can share characteristics common in several architectonic styles. Despite their appearance on the different styles, a window, a door and a wall are transversal elements 
to the majority of the existing architectonic styles. The representation of particular cases (eg. Manueline window, mesquite entrance, skylight) are extensions of the generic ones.

"Shared ontologies let us build specific knowledge bases that describe specific situations."

- Chandrasekaran et al. (1999)

Besides re-usability, ontologies are considered, by Chandrasekaran et al. (1999), abstract structures. The combination of these two features are completely compatible with the design of data models and object oriented programming classes (towards the considerations made by Guarino (1998)). Thus, the ontological analysis of a certain domain field can be suitable for integration in areas such as computer science and software engineering, for both project stages: requirement analysis and algorithmic development.

Considering the notions left by Guarino (1998) and Chandrasekaran et al. (1999) at an abstract level, one can infer that the use of ontologies can be extended to other areas - besides information systems or software engineering - namely the ones involving virtual representations, as some authors have already shown (e.g. Jannin and Morandi (2007), Attene et al. (2009) and Béhé et al. (2014)). The following subsections will expose some of the works that successfully applied ontologies to regulate virtual representations in different scenarios and contexts. CityGML standard will also be discussed, due to its relevance in the context of virtual environments representation.

\subsubsection{Virtual representations based on ontologies}

The integration of ontologies in some of the works developed around virtual representations will be presented in this subsection. Although the concept of ontology has been explored for a while, this way of structuring knowledge is still largely used by several authors today. 
Pittarello and De Faveri (2006) were concerned with the lack of semantic description across the considerable amount of virtual environments available on the Internet, which prevented a proper interaction with them. To get around this issue by enhancing the models with semantics, the authors proposed a solution capable of relating geometric primitives - X3D and VRML - with semantic class objects through the so called MetadataSet nodes. An independent scene ontology was also incorporated to establish a set of relations used for the description of a certain domain (for example, a wall can be contained inside the room).

One year later, an approach involving surgical models for computer-assisted neurosurgery was proposed by Jannin and Morandi (2007). The aim of this work is to provide a visual framework for planning surgeries, improving human-computer interfaces - specifically, for the computer-assisted surgery systems - thus formalizing the surgical knowledge and practice. The presented framework relies in a surgical ontology which establishes the concepts and relationships belonging to the surgical work domain - extensible to other areas of medicine - and a supporting software that describes the surgical procedures.

For setting up urban environments, a work that employs ontologies was proposed by García-Rojas et al. (2008). Their parametric system allows common users - nonexperts in the virtual reality field - to prepare 3D scenes through an on-demand configuration. To achieve this, a visual programming paradigm, supported by a proper ontology, allows the organization of a 3D scene components.

A pervasive system, proposed by Lee et al. (2008a), combines ontology-based contextawareness with adaptable augmented reality. In short, the framework considers several aspects such as user preferences, device profiles and security to augment personalized virtual models, which must in accordance with a given acquired context. Context-awareness is provided by three ontologies. The first ontology holds the general concepts related with the pervasive environment. A second ontology organizes knowledge about users' device profile (related with the mobile device capabilities) and preferences (a set of user options). At last, a social ontology maps users activities related with information shared in the web, for further re-utilization. The authors exposed three system applications as examples: ubiquitous home visualization and 
simulation, ubiquitous car services and ubiquitous engineering collaboration.

The strength of ontologies was once again highlighted by ShapeAnnotator: a system developed by Attene et al. (2009) that allows the classification of 3D virtual model meshes in a certain knowledge domain. The system provides a set of tools for model segmentation that can be manipulated by a user in order to easy and properly link the model parts to the domain knowledge, formalized by an ontology. The usefulness of ShapeAnnotator framework was discussed in two scenarios. The first one explains the potentialities of the tool in supporting the creation of human models (avatars) for Multi-massive online role playing games (MMORPGs) and virtual worlds, such as Second-Life. The second scenario focuses the collaborative e-manufacturing of $3 \mathrm{D}$ products taking advantage from the abstractness and re-usability provided by the ontology.

In the museums context, another system assisted by ontologies that aims the collaborative annotations of 3D museum artifacts through web-based services, was proposed (Hunter and Gerber, 2010). This system - entitled Harvesting and Aggregating Networked Annotations (HarvANA) - promotes the participation of communities in the cultural enrichment and improves museum objects indexation. One of the most relevant features of this system is the flexible ontology-based categorization - called of folksonomy - which optimizes tagging proceedings among the communities.

In the context of urban planning and management, Martins (2011) proposed an urban ontology to overcome the issue of data heterogeneity among municipalities that use different geographic information data sources. The main idea is to establish a common data model and provide an unified platform for data sharing between municipal technicians. Thereby, the author supported a significant part of his work in the CityGML standard - addressed in the next subsection - to develop a set of data models, which intend to reflect the different urban elements. For example, the proposed building model establishes a structure which includes a building, building parts, rooms, openings such as doors and windows and several boundary surfaces like walls, grounds, ceilings and furniture. 
A couple of years later, Colledani et al. (2013) proposed the integration of several heterogeneous software tools for manufacturing activity design over the same platform, using ontologies for uniformization purposes. This is implemented through the socalled Virtual Factory Framework, which is composed by several components: data and knowledge, Semantic Virtual Factory Data Model (VFDM), Semantic Virtual Factory Manager (VFM), decoupled virtual factory modules and the real factory interface. In this case, the VFDM is the abstraction layer holding the ontology that can extend products and define manufacturing processes. The rest of the components complete the system by ensuring the connection of the framework to the external applications, through special framework connectors designed for integration.

Recently, Flotynski (2014) developed the Semantic Modelling of Interactive 3D Content (SEMIC), which employs a method for the modelling of knowledge, rather than the modelling of virtual content itself. SEMIC employs a method that consists in the mapping of 3D content into semantic classes, which are related with each other, in order to establish object relations for a given domain. The creation of 3D content consists in a set of well defined steps that includes the design of the concrete semantic representation containing properties related with the 3D content, the mapping of specific domain concepts and the design of a conceptual semantic representation of 3D content, for arbitrary 3D creation purposes. These steps, as the author refers, require the intervention of different skilled professionals such as content developers, domain experts and content consumers. This aspect might suggest that this system is somewhat complex regarding $3 \mathrm{D}$ virtual models creation process.

Another recent approach addressing simulations in virtual environments was proposed by Béhé et al. (2014). The authors presented a framework for interactive multiagent-based simulations in virtual environments, adapting ontologies as a core notion to ease the simulation design and re-usability. Thereby, simulations are configurable through semantic modelling. This is used to describe the different aspects of the simulation, namely agent behaviours, surrounding environments with physical objects, scheduling for operation progress and the results of agent actions and interactions. 
The differences between Geographical Information Systems (GIS) and BIM were addressed by Mignard and Nicolle (2014), who developed a system called SIGA3D. This system takes advantage of ontologies, to provide interoperability between construction and urban management. So, information about buildings and geographic data can be managed together, in the same structure. The system also promotes collaboration between facility managers, aiming the enrichment of knowledge models since the designing stage to buildings' recycling .

The last work reviewed in this subsection is Virtual Collaboration Arena (VirCA): a collaborative virtual/augmented reality framework that enables testing and training events in the context of manufacturing systems, through several practical scenarios (Galambos et al., 2014). Such scenarios are mounted through web-based applications and interfaces that provide mechanisms for using and extending virtual reality content. Interaction with the referred scenarios is provided by cyber devices, also known as CDs, that enable the manipulation of their objects. The ontology concept acts here: a semantic manager layer ensures the bidirectional communication between CDs and virtual scenes, factoring on the available capabilities and requests - in terms of allowed actions/functionalities - supplied by the scenes' ontologies.

Summing up, one might conclude that ontologies have made a significant contribution to the success of several works, some of them referred in this subsection. This way of organizing knowledge to regulate processes has revealed robustness and flexibility in several works that require data representation through virtual models in a wide variety of contexts such as simulation, industrial manufacturing, collaboration, urban planning and others. However, in the specific context of 3D urban modelling, there is a well-defined urban knowledge structuring standard named CityGML. The next subsection will address some of the most relevant features of this standard.

\subsubsection{CityGML: a 3D urban environment standard}

CityGML is, perhaps, the most important effort for the standardization of 3D urban representations (Gröger et al., 2008, 2012). These guidelines, proposed by the Open Geospatial Consortium (OCG), intend to provide a widespread XML-based format 
for the geometric and semantic representations of city components. Kolbe (2009), one of the co-founders of CityGML, explains this standard, starting by describing the scheme of thematic classes (Figure 2.1) that is composed by an abstract base class - _CityObject - from which all of the other thematic classes inherit, for example, CityModel, Site, _WaterBody, _Vegetation,_AbstractBuilding. It is noteworthy that these classes are conceptual structures isolated from the geometric aspects, which are addressed by the Geometry structure associated to each conceptual class. Geometry extends a set of primitives for representational purposes which intend to describe geometrically the objects spawned from concept classes.

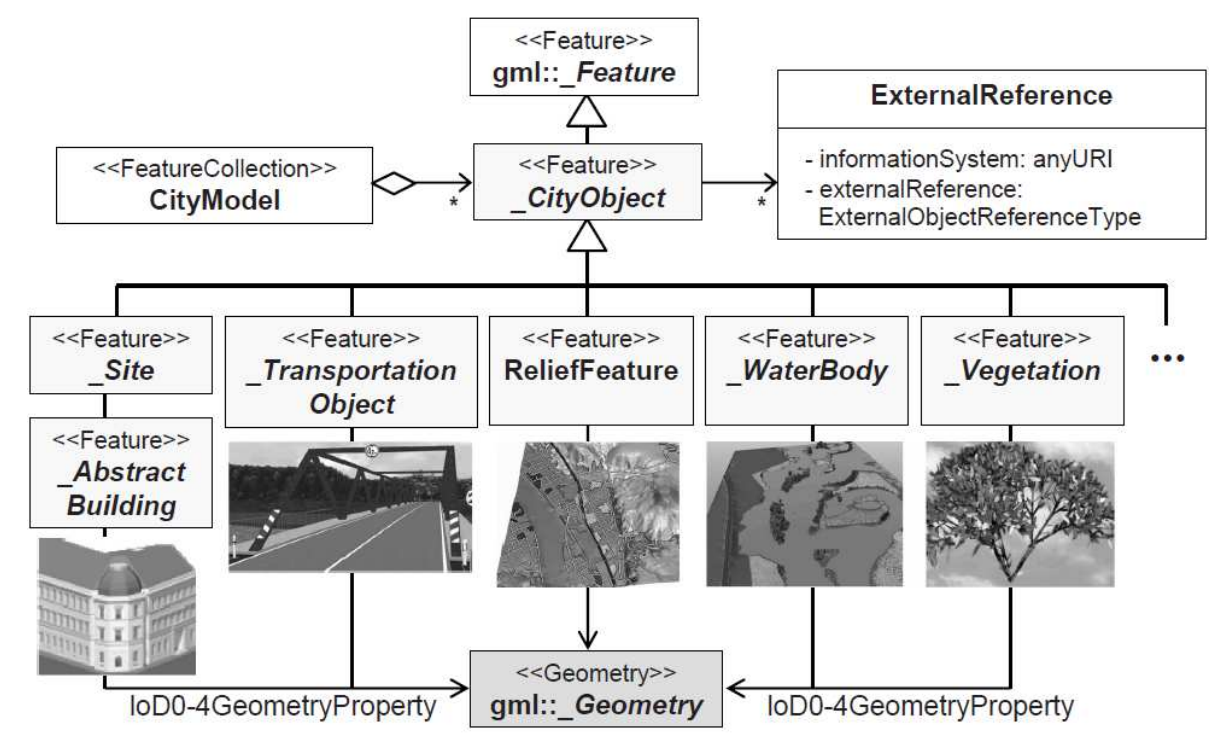

Figure 2.1 - A UML diagram representing the top classes of the CityGML standard (Kolbe, 2009) in which objects are considered features and their classes are derived from _CityObject.

The term "3D urban environment" can lead to the idea that only the exterior aspects of a city are addressed. However, the standard also covers the building element and both its inner and outer components. Kolbe (2009) explains the CityGML support to this crucial city element, by presenting also an abstract class named of _AbstractBuilding. This is the mother class that derives to Building and BuildingPart. Building holds sets of BuildingPart which can be seen as groups of structures that take advantage of the recursive relation with the _AbstractBuilding in order to support a wider range of structural rearrangements. For example, a given building 
may hold a stack of floors and a castle might be constituted by a set of horizontal distinguishable parts such as towers, curtain walls or gatehouses. Buildings and building parts can be represented in terms of constraints by another important class which is the BoundarySurface. This one can derive to specific boundaries such as WallSurface or RoofSurface. Furthermore, this boundaries may hold a set of objects spawned from _Opening class, as for example, doors and windows. Finally, Room class is intended to support the inner compartments inside the building and building parts. The diagram that describes the building knowledge organization is depicted on Figure 2.2.

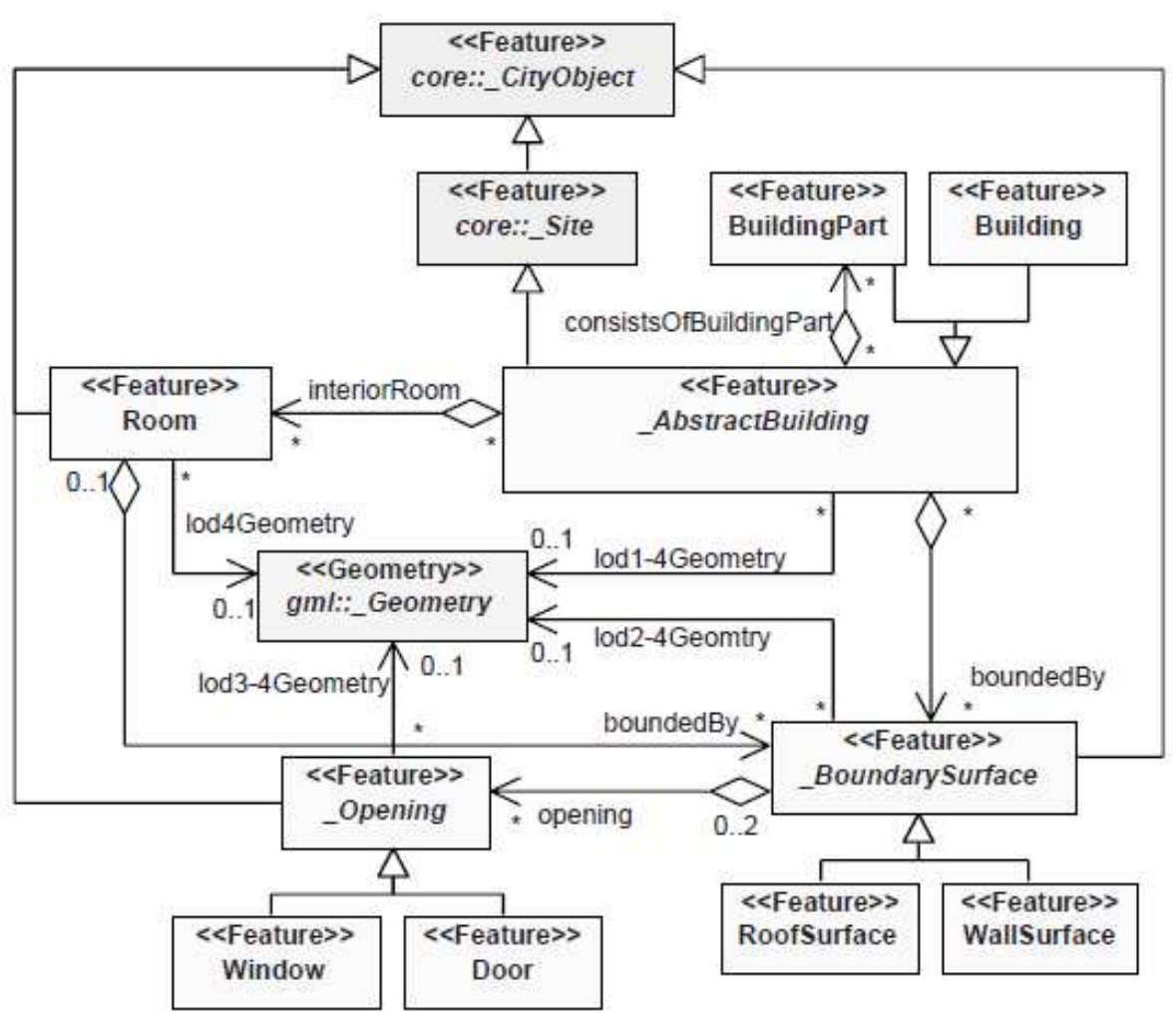

Figure 2.2 - UML diagram showing a simplified excerpt from the CityGML building model (Kolbe, 2009).

The specification of buildings is also extensively addressed by Industry Foundation Classes (IFC): a data model standard widely used by the construction industry for project purposes, in the context of BIM process (Liebich, 2013). However, 
the quantity of addressed civil construction and architectural domains (BuildingSMART, 2013) is excessive and technically complex to describe an urban environment oriented for areas such as videogames, 3D cinema or archaeology. The overload of dispensable information - not perceptible to the eye during representation - can even, in some cases, harm the objective of the virtual building representation, depending on usage context (unnecessary heavy and time consuming renderings, graphical lag, heavy processing, etc.). To conclude and considering such aspects, CityGML seems to be more suitable for designing general urban environments that target virtual content creation for areas as the ones that were aforementioned.

Besides the various applications of ontologies on virtual environments, some procedural modelling works also use them to regulate structures' generation (e.g.: Trescak et al. (2010); Liu et al. (2008); Yong et al. (2012); Liu et al. (2014); Bellotti et al. (2011)). The aforementioned works will be presented during the following pair of sections, which are reserved for an extensive analysis to procedural modelling, including the generation/reconstruction of virtual extensive urban environments and traversable buildings.

\subsection{Procedural Modelling of Virtual Urban Envi- ronments}

In this section, the production of virtual environments concerning the generation/reconstruction of exteriors will be presented. These environments are mainly composed by street networks and buildings, exclusively represented by outer facades.

\subsubsection{L-systems for procedural modelling}

L-System was introduced by Lindenmayer (1968) and adopted by Parish and Müller (2001) to generate an extensive virtual environment considering its exterior layout. This technique - depicted on Figure 2.3 - uses an alphabet of symbols combined with a set of production rules. The process starts with an initial set of symbols 
that are iteratively replaced by other symbols until the final string is obtained. This final string is then used to generate shapes through a transformation mechanism. Parish and Müller (2001) used this technique in two steps: one for generating a street network and other for producing buildings that can be described by several formats, provided by extrusion operations.

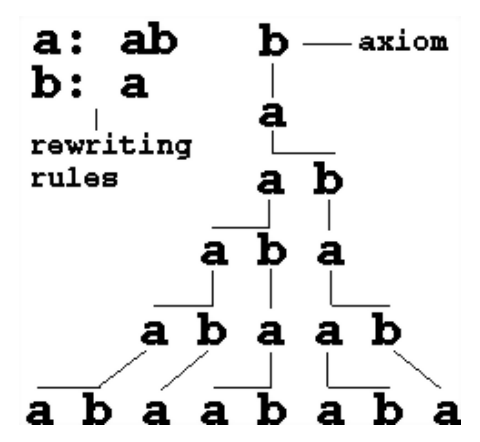

Figure 2.3 - L-System operation: an axiom is the trigger element which is then replaced accordingly with re-writing rules. The resulting symbols are once again replaced accordingly with the referred rules and this process is repeated until the final string is obtained (pyramid bottom).

XL3D modelling system (Coelho et al., 2007) also incorporates a geospatial LSystem. Streets and blocks coordinates provided by a database populated with real data were used in combination with the L-System production rules, to generate the virtual downtown of Porto, Portugal.

\subsubsection{Detailing facades through split grammars}

Split grammar was introduced by Wonka et al. (2003). This technique relies on a grammar that operates in the context of a shape in order to produce 3D layouts. There are two types of rules in this grammar: splitting rules to replace geometries and conversion rules to produce transformations upon geometries. Shapes marked to be splitted or converted can be categorized in two classes: terminal and non-terminal. The process starts with an initial shape. A split grammar operates iteratively to force the shape to undergo several non-terminal states, until its final form. This process is considered finalized when all shapes in a pool reach their final 
state. Figure 2.4 depicts the aforementioned process. This technique was applied to improve the details of building facades.

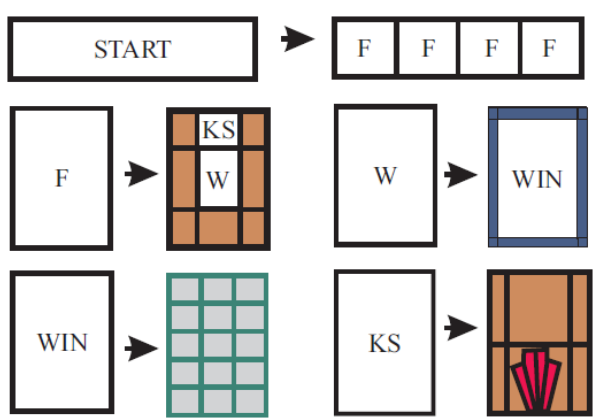

Figure 2.4 - Set of operations performed by split grammar (Wonka et al., 2003): an initial shape undergoes a process of consecutive splittings and replacements to detail a certain element (in this case, a window). The process ends when a final shape is reached, that is the same to say that there are not more shapes to split or replace.

Later, Larive and Gaildrat (2006), developed a wall grammar for buildings' generation, based on the the split grammar of Wonka et al. (2003). This wall grammar integrates a technique to produce 3D buildings with exterior facades. The process starts by importing a given building footprint and height from a GIS that occurs automatically or by considering user specifications. Then, a building template is assigned to the outlines to define high-level features for the building exterior appearance. This assignment can be performed manually, randomly or even based on social-statistical data. An important condition to be observed in the referred building templates to be assigned is that they must be geometrically compatible with the building frontage. Such templates also store references for primary wall, default material and user-defined keywords that can be used, for example to identify the main segment that holds building entrance. Afterwards, a set of grammar rules is applied to create the exterior facades which results in a building with extruded walls. Differently from Wonka et al. (2003), this grammar operates on walls instead of shapes. Finally, a straight skeleton technique (Felkel and Obdrzalek, 1998; Eppstein and Erickson, 1998) is used to generate the building's roofs. 


\subsubsection{Semi-automatic digital reconstruction of old buildings considering GIS data-based topology}

A preserved area of Nicosia city was digitally reproduced through a partial automatic method which combines geographic data of building bases, building classification and style-concordant building components, such as doors and balconies (Dikaiakou et al., 2003). Two distinct processes integrate the method. The former consists in photographing particular elements of the city to model realistic virtual building components. The later is a rule-based automatic process that starts by comparing the outline of each building - obtained from GIS data - with templates to determine building topology. Then, the ground edges are transformed into 3D walls. The previously modelled virtual building components are applied to each wall accordingly with the building topology classification and wall space available. Lastly, using a straight skeleton computation approach (Aichholzer and Aurenhammer, 1996; Felkel and Obdrzalek, 1998), the roof of each building is properly produced and applied.

\subsubsection{Random extrusion of floors}

Greuter et al. (2003) proposed a system to generate pseudo-infinite cities. The generation of road networks is based on a regular grid, globally adjustable. This system also involves buildings' generation. Thus, in each grid block (area between streets) and using a hash mechanism, a random number is seeded to provide building properties such as height, width and number of floors. This location-based virtual building generation is endowed with a permanent recollection of the building, that is always generated with the same appearance, even in further re-visitations. Moreover, to generate such buildings, the authors opted for combining geometric primitives each set corresponds to a floor plan - that varies from floor to floor. The process starts with an elementary set of geometries, at the building top. Then, an extrusion forms a volume and primitives are added to a subsequent level. The process is repeated until reaching the building ground level. As it can be seen on Figure 2.5, the extrusion process starts with the most basic set of shapes and ends with the most complex one. 


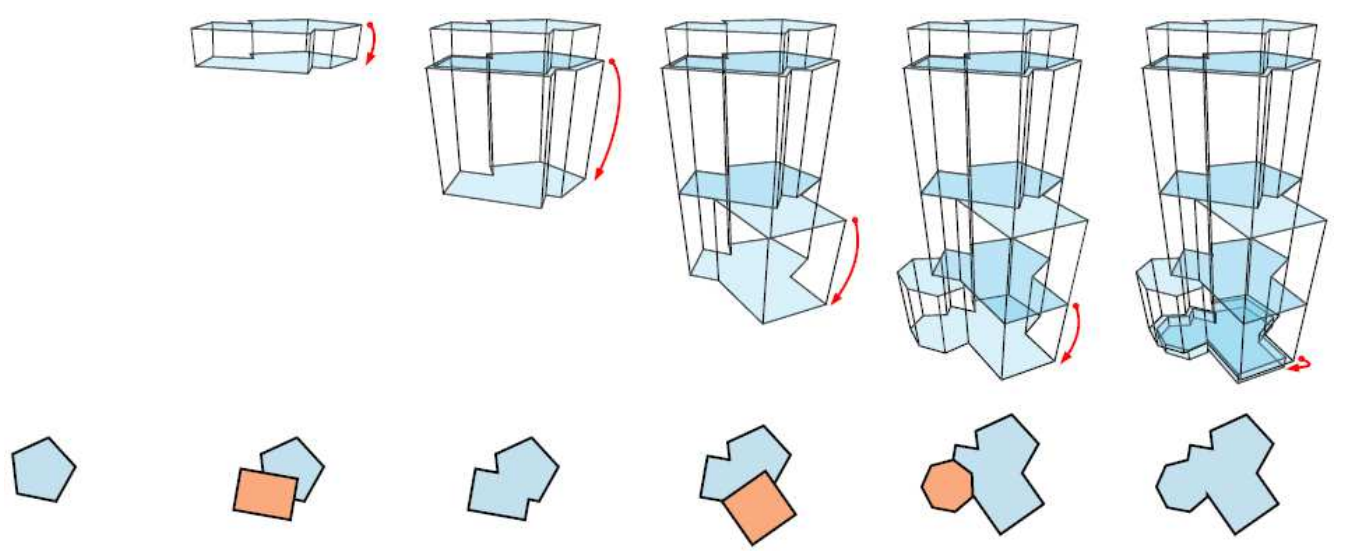

Figure 2.5 - Process of floor extrusion proposed by Greuter et al. (2003). It starts by extruding the most basic combination of shapes and ends with the most complex one, from the top of the building to its base.

\subsubsection{Feature-based decomposition of facades}

Finkenzeller et al. (2005) were concerned with the structural heterogeneity of buildings namely to possible significant differences in building facades. These differences are related with the facade format - given by the arrangement of its components, as for example, doors and windows - or with the presence of complementary elements such as balconies, orioles or bay windows. Thereby, they proposed a system capable of dealing with the referred heterogeneity through the decomposition of structures into convex polygons called floor planned modules (fpms). Fpms can be seen as aggregations of ground marks to describe a building morphology, regarding also structural elements (e.g. balconies). With this concept the authors were capable of producing buildings with highly detailed realistic facades. Multiple floors are also supported. The referred work preceded another system that produce buildings with complex facades, proposed by (Finkenzeller and Schmitt, 2006). In this system, designers must provide high-level requirements such as building coarse, type and style to produce highly accurate 3D buildings. They can also change facades parameters after building's generation, as the system is prepared for recomputing such modifications. A more mature version of this later system was presented by 
Finkenzeller (2008) and it was used to produce the virtual model of the University of Karlsruhe. The realism of the exposed models is impressive. However, such level of detail requires spending between few minutes to about two hours per model, accordingly with the author.

\subsubsection{Computer generated architecture for buildings pro- duction}

Computer generated architecture (CGA) is another methodology related with outer facades generation, which relies on a rule system provided by shape grammars (Müller et al., 2006b). In short, a mass model, composed by volumetric shapes (Figure 2.6), constitutes the starting step of the process. Afterwards, the outer facades are generated, resulting in the building's exterior appearance. Finally, the doors and windows are placed. The whole process ensures a high-level of coherency, avoiding unnatural occurrences such as truncated windows. The grammar used to operate respects the following format:

$$
i d: \text { predecessor : cond } \Rightarrow \text { successor }: \text { prob }
$$

As it was referred, the process initiates with the creation of a mass model that constitutes the exterior format of a building, including the roof. This mass model can be seen as a merge of volumetric shapes that can variate in scale, rotation and usage portion. In the next step, facades are created to properly cover the mass models. The final step of the process increases the detail in doors and windows and also accommodates building ornaments. The grammar used in this technique - an extension of the split grammar - is sequential (similar to the Chomsky grammars addressed by (Sipser, 1996)) and performs transformations directly in the geometry, allowing to operate with shapes or its components. This is suitable for modelling at 1, 2 or 3D. In their work, authors presented tests made with CGA shape grammar which was incorporated in City Engine framework. This grammar enables a largescale production of buildings with different styles. A suburbia model of Beverly Hills was produced and depicted along with a procedural reconstruction of Pompeii, 
generated with 190 manually encoded CGA shape rules.

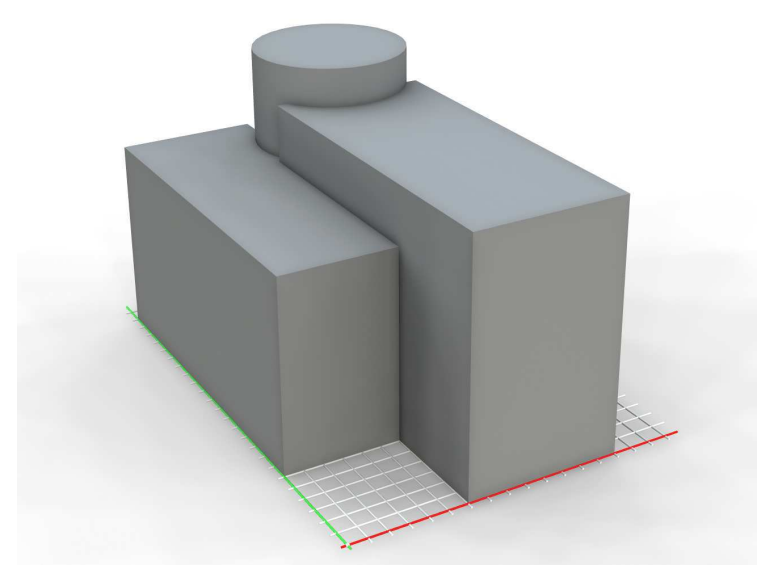

Figure 2.6 - Building mass models are created from the merging of volumetric shapes at the beginning of the process. Müller et al. (2006b) use this grammar to successively operate upon mass models in order to produce highly detailed and realistic virtual buildings.

CGA was also applied in some works aiming virtual reconstructions (Dylla et al. (2009), Müller et al. (2006a), Besuievsky and Patow (2013), Tepavčević and Stojaković (2013)). Dylla et al. (2009) developed a system to produce a 3D reconstruction of ancient Rome (Figure 2.7) through the combination of manually designed structures and procedurally generated buildings, in the same virtual environment. The class of each element defines what kind of approach is needed. For known positions, dimensions and design, class I elements are loaded from models created using a commercial computer aided design (CAD) software. If some information is missing, class II elements are generated procedurally using CGA shape methodology.

The same methodology was also applied by Müller et al. (2006a) with the purpose of reconstructing Puuc-style buildings, that are similar structures to the ones found in Xkipché, México. In their work, the authors created a grammar to fulfill the architectonic requirements of the referred buildings. Thus, accordingly with the typical design of these structures, the grammar defines the building as following: firstly, the base is defined; then, middle walls are addressed considering building accesses; upon these walls, a middle-layer designated by medial molding is produced; the last rules define frieze, cornice molding and completion ornaments. 


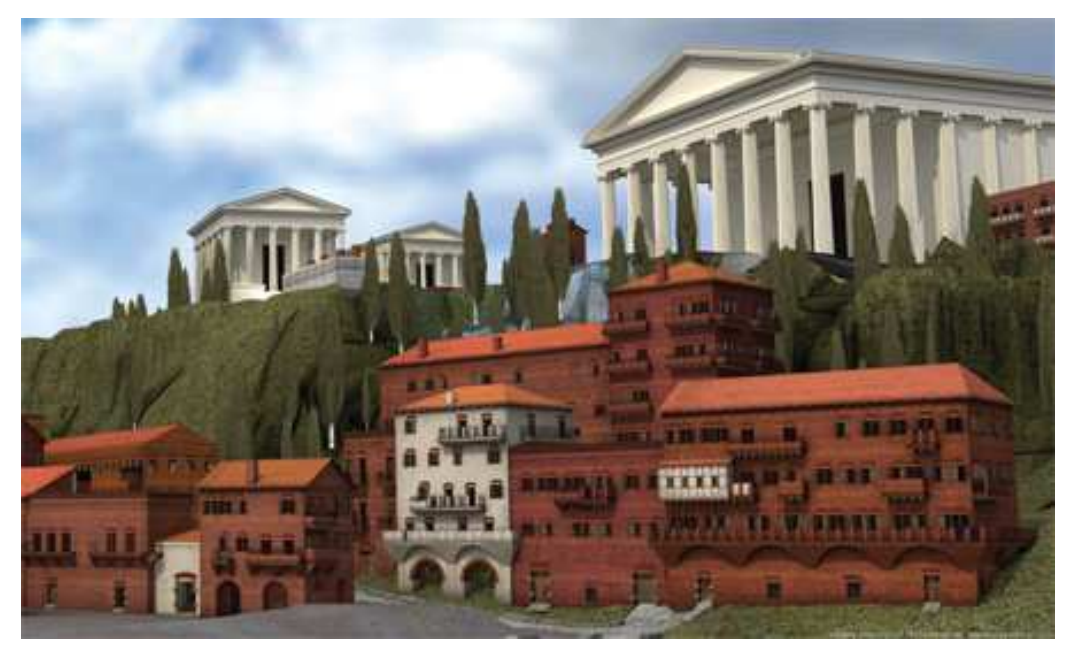

Figure 2.7 - Virtual Rome produced by the system of Dylla et al. (2009). This city virtual model contains class I models that were manually produced and also class II models, procedurally generated with a CGA shape rules set in order to overcome the lack of information.

Besuievsky and Patow (2013) developed their own CGA shape rules to reconstruct historic buildings and urban environments for serious games. They use as input 2D data provided by GIS with corrective mechanisms to deal with map issues, like distortion. This input allows the acquisition of relevant features such as building outlines, for further extrusions, forming mass models. The production of building facades is made through a user-friendly tool which hides the grammar to improve the easiness of use. The application of this methodology produced some interesting results on the virtual reconstruction of Carcassone's old town, in France. The tool's flexibility was demonstrated through the virtual reconstruction of two other cities with different architectonic styles: Nantes of France and Girona of Spain.

Tepavčević and Stojaković (2013) combined fuzzy and statistical analysis with CGA shape to generate Neo-Gothic chapels. A set of sacral Roman Catholic buildings from the Danube area of Vojvodina was selected. These 86 elements - built in the period from early 18 th to the middle of the 20th century - were statistically analyzed in order to extract distinct characteristics. The resulting analysis, that considered parameters such as purpose, location, building period and shape characteristics, enabled the isolation of a fuzzy set composed by Neo-Gothic chapels built around the year of 1900. According to the authors, fuzzy logic can be applied to architecture in 
order to deal with the uncertainty introduced by the influence of different styles in the buildings. Taking into account such possibility, they developed a reliable mathematical model that combines fuzzy logic and probabilistic calculations to produce stochastic CGA shape rules, that are used to generate realistic Neo-Gothic chapels featured with the style covered by the referred fuzzy set. Each set of rules defining a chapel starts by specifying a building lot and a mass model. The mass model is decomposed in three main parts: apse, nave and tower. Apse and tower mass model parts are replaced with appropriate shapes and the subsequent steps will detail the model until the final 3D form.

\subsubsection{Building generation based on facade view acquisition}

A work focusing the decomposition of building facades through image analysis and shape grammars was proposed by Müller et al. (2007). Their system starts by processing a facade image and extracting its elements using automatic operations, through a top-down hierarchical process. For example, a given facade image is divided into floors which are, in turn, subdivided into tiles that are then partitioned into smaller rectangles. Afterwards, a stage that consists in matching the last rectangles with a library of 3D architectural elements, takes place. The whole process results in a tree shape that is encoded into a shape grammar, which holds the definition for facade representation.

A similar research line was followed by Koutsourakis et al. (2009), who proposed a framework capable of producing 3D models from a single facade image. Its inputs are a parametric shape grammar and a rectified image of a single building facade. A tree-based process takes over the generation, collecting a set of rules that regulate it. The concrete geometry is not a major concern for the tree structure. There is also a parametric grammar that provides some additional data during the tree crossing, that includes, for example, the definition of a building weight or even widths for windows and facade patches. Then, a Markov Random Field (MRF) formulation optimizes the parametric rules to produce the buildings' final aspect. Each rule can be seen as a simple MRF which consists in a set of nodes that represent a basic shape. 
This is defined considering label sets and associated costs. MRF formulation is used to split the building horizontally and vertically and also for extrusion purposes. Later, Simon et al. (2011) developed a system that uses shape grammars and facade image classifiers to generate 3D buildings. A combination of procedural modelling, statistics and image processing led to their solution. Both of the previously referred works are extensively addressed on Simon's PhD thesis (Simon, 2011).

\subsubsection{Digital map-based generation of 3D buildings with multiple roofs}

Sugihara and Hayashi (2008) proposed an automatic solution focused on the production of virtual buildings with multiple roofs, considering building footprints provided by digital maps. Using a system to express polygon angles and sort vertices (clockwise), their method is capable of splitting a building footprint into rectangles, that are used to determine roof branches and constitute the base shape for roof creation. The presented results demonstrate a set of buildings automatically generated, each one holding a set of gable roofs unified by branches. Moreover, complex roofs were also produced in order to fulfil the requirements for the reconstruction of an ancient japanese temple and also a pagoda.

\subsubsection{City Modelling Procedural Engine (CMPE)}

Carrozzino et al. (2009) developed an engine that uses a set of input elements such as aerial photographs, vector and raster maps or even text descriptions, to produce extensive urban environments. The process to attain urban environments includes a set of steps that can be validated one by one, by the user to avoid the generation of inconsistencies or requirements failure. At the beginning, streets data and block footprints are automatically extracted from the input maps. Then, a 2D road network is produced, followed by its 3D representation. 3D blocks and related buildings are subsequently generated. The buildings are produced considering the angles formed by the blocks' constraint perimeter. Dimensions - size and height - 
are applied based on the specifications provided on the city graph definition (user validation during steps crossover). The absence of these specifications will produce general purpose buildings with pseudo-random dimensions, that can be customized by the user. Besides the code-based procedural generation, an authoring tool was also developed to assist the user in the manual interventions, aiming the refinement of the virtual buildings. This tool also allows the creation of complex models with a few input parameters. Texturing features and geometrical components can be also applied for models enhancement purposes. A clean visualization of the whole scene is provided through the XVR rendering engine, incorporated in the CMPE.

\subsubsection{Procedural generation 3D (PG3D) solution}

A solution for procedural generation of extensive urban environments was presented by Silva and Coelho (2011). They opted by a strategy that consists in storing the instructions for modelling urban elements and geographic data in the same spatial database. The instructions for buildings' modelling are stored in database native language, pointing to the shape grammar rules. Each set of these rules can be seen as instructions to guide the geometric generation process of elements such as buildings, roofs or balconies. Moreover, this solution distinguishes the different layers where elements are placed: surface, roads, buildings and so on. The boundaries that specify layout variations - city center vs. suburbs - are also supported through grammar. Lastly, a tagging system for the direct selection of geometric components (e.g. a face of a certain building), allowing possible transformations or improvements is presented. The presented results demonstrated the modeller capabilities in generating large-scale urban environments: a 3D model representing $10 \mathrm{Km}^{2}$ of Porto (Portugal) - including Boavista (Porto, Portugal) roundabout, Aliados avenue and Porto downtown - was successfully produced, with a considerable degree of resemblance (see Figure 2.8). Some exporting mechanisms were also implemented, aiming the models integration in virtual worlds, for gaming or simply exploration purposes. The extended version of this work can be found in the master thesis of Silva (2010). 


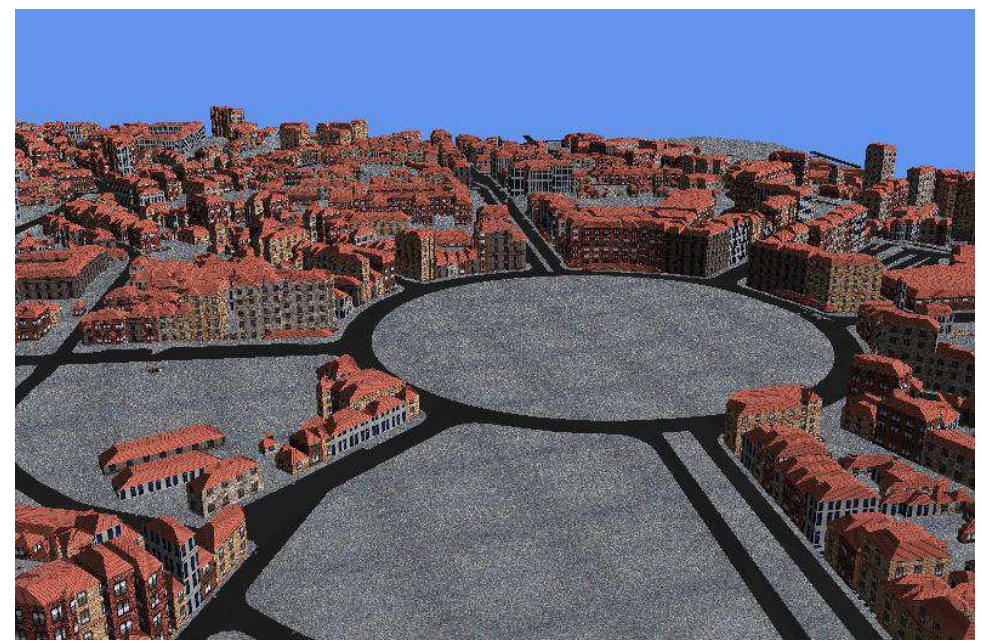

Figure 2.8 - Boavista roundabout produced by PG3D (Silva and Coelho, 2011). An available set of information was considered to generate the virtual model of this urban area, that has considerable degree of resemblance.

\subsubsection{Ontology-based generation of urban environments and building exteriors}

The generation of ontology-based virtual urban environments was an area that few explored. In the current subsection, these works will be addressed.

\section{Ontology-based procedural modelling to recover cultural heritage}

An ontology-based solution was proposed by Liu et al. (2008). The authors embraced the challenge of recovering the cultural heritage of ancient China. To accomplish such challenge, a city generator was developed, capable of producing virtual models based on an ontology and on user input: a grammar for building definitions. Moreover, a style checker was implemented to avoid generation inconsistencies, such as buildings upon streets. Their work is also one of the few cases of an extensible ontology application that covers other architectonic styles. Some results of virtual buildings with outer facades were presented regarding both indian and chinese architectures. This system followed their previous work (Liu et al., 2006) in which a semantic-based modelling system was proposed. The objective was to improve 
users' focus on its specific implementation, while the geometric details are encapsulated by the semantic elements such as walls, doors and windows. In short, a user propagates the semantic information of a building using a XML format and then a document type definition verifies the XML conformity. Finally, in case of success a procedural modeler produces the geometry according with a user demands.

Recent works (Yong et al., 2012; Liu et al., 2014) include some additional features. Yong et al. (2012) reported the improvements made to the previous semantic-based solution ( $\mathrm{Liu}$ et al., 2006) that intended to overcome some noted issues regarding procedural modelling, such as the lack of annotations for digital architectural heritage which also impacts in the identification of procedural rules for digital reconstruction of missing monuments. Such issues triggered the proposal of an approach that puts together semantics, machine intelligence, data mining and automatic annotations. Later, a granular ontology approach was suggested by Liu et al. (2014) to allow a collaborative ontology design based on the sub-concepts provided by users of different expertise areas.

\section{Semi-automatic generation of ontology-based building facades}

Bellotti et al. (2011) proposed a statistical algorithm for the procedural generation of urban areas, capable of producing buildings composed by several ontology-based facade components. The starting point of the algorithm is a $2 \mathrm{D}$ vectorial map with georeferenced building bases. These bases consist in closed polygons that are extruded at a certain height and then have each lateral facade element decorated with textures statistically selected from template styles. While, facade elements are mapped in an ontology that organizes and relates several architectonic elements such as windows, doors or roofs - from a semantic viewpoint, template styles are manually indicated by the modeller, that selects areas of style on the $2 \mathrm{D}$ map. The authors used the algorithm for the generation of urban environments in the context of cultural heritage promotion and in a 3D movie. Both were presented to users who rate positively the reconstruction, despite the absence of architectonic details, provided by elements like balconies or porches. 


\subsection{Procedural Modelling of Virtual Traversable Buildings}

Besides the focus on urban environments and buildings outer facades, several other approaches address the procedural modelling of 3D traversable buildings. In the next subsections, they will be reviewed.

\subsubsection{LaHave House: an automated architectural design ser- vice}

Rau-Chaplin et al. (1996) developed an automated architectural service to provide a collaborative way of plan and design modern houses, foreseeing the interaction between architects and final users (clients or service consumers). Their system is constituted by three components. The first one, design engine, provides libraries of house designs to the final user. These designs - that differ in organization, size and style - are generated through an intrinsic shape grammar that defines the possible house sections (public, private and semi-private) and related rooms. Currently, their design engine generates over 100000 different house designs. The second component allows the selection, customization and visualization of the designs provided by the library. These operations are made by the final user. Lastly, the customized designs are turned into working/assembly drawings through the third component: a building configuration tool.

\subsubsection{Procedural generation of buildings using graphs and expansion algorithms}

Martin (2005) presented a procedural algorithm to generate residential units, based on architectural insights. Considering a grammar and user-defined constraints, the process starts by generating a room graph. Each node corresponds to a room and each edge represents the connectivity between room pairs. The first rooms added to the graph are public rooms. Then, each room receives a specific type (living 
room, dinning room, etc.). Private rooms are also added and then, stick-on rooms such as closets and pantries are attached to them. When the graph is complete, the process's placement step spreads the rooms over the footprint. Lastly, using a Monte Carlo algorithm, the rooms are properly expanded until equilibrium is reached. This expansion occurs considering wall pressures used to make rooms expand or shrink. Figure 2.9 depicts the result of the author's approach. In its thesis, Martin (2006) discusses graph-based techniques to generate structures of connected rooms and to place them within a given area. The process of room expansion is also explained in detail. Regardless of the similarities with the Monte-Carlo algorithm, it seems that this designation was replaced by square bubble growing algorithm.

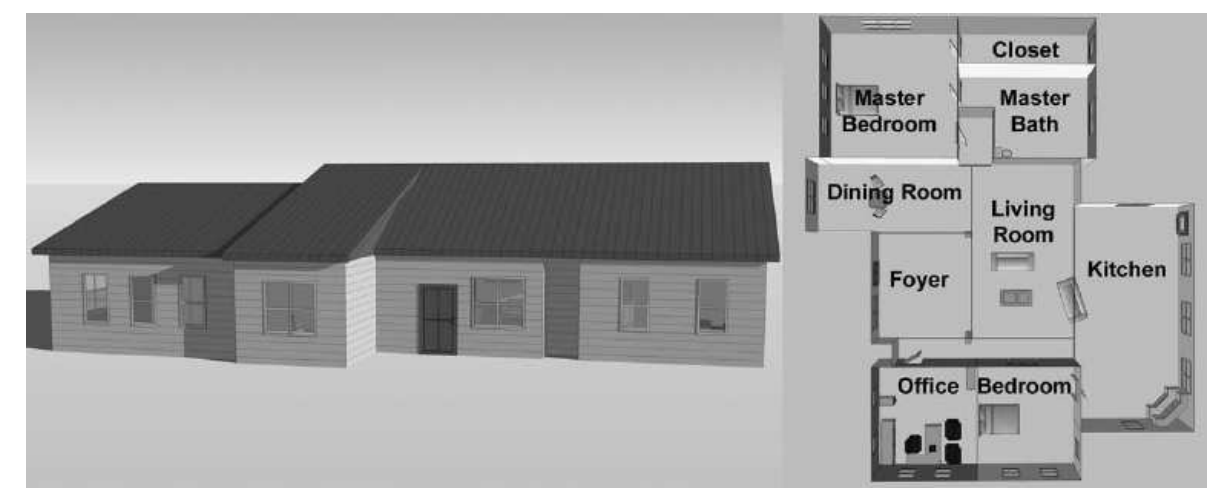

Figure 2.9 - An house with interiors produced by Martin's approach (Martin, 2006): firstly, a graph system is used to connect and place rooms and then those rooms are increased in a fixed area using a Monte Carlo algorithm.

A similar solution - based on seed and growth approach - was proposed by Long (2011). This system considers as input an area for feature placement that can be rectangular or non-rectangular. Still, it is confined to shapes formed exclusively by right angles. The process of area fill includes the determination of feature types (shape variety) and also their placement and adjustment in the available space. The author argues that his technique is more effective than squarified treemaps approach, that is addressed in a later subsection. However, the algorithm does not show how to achieve a fully connected floor plan or even a 3D representation of the virtual building model. 


\subsubsection{Building indoors generation using constructive solid geometry algorithms}

Bradley (2005) identified the main issues related with the production of videogames requiring extensive urban environments, at that time. Most of them did not offer the possibility of exploring building interiors. On the other hand, the production of indoor spaces requires a lot of human resources and time. To tackle with such constraints, he proposed a semi-automatic methodology to produce traversable buildings which considers two types of input: American Standard Code for Information Interchange (ASCII) files with heuristics and room definitions and building outline. Some 2D geometrical transformations divide the outline into empty rectangular cells of variable size. Then, the rooms are properly distributed upon the cells using a recursive approach. The walls are extruded and then pierced to hold windows and doors, considering methodology rules. For example, if an overlap between two walls is verified, they are treated as a common wall, shared by two rooms. In this situation, a set of geometric transformations are performed to place a door between the referred adjacent rooms. The aforementioned geometric transformations are managed by 2D and 3D Constructive Solid Geometry (CSG) algorithms. The outputs results are $3 \mathrm{D}$ traversable buildings devoid of any details.

\subsubsection{Lazy production of virtual building interiors}

The concept of real-time generation of interior divisions was exploited by Hahn et al. (2006). The plans of each floor are generated through a random division of the floor into rectangular divisions and hall passages. The division process starts by defining a temporary region which is then divided in smaller temporary regions and built regions. This is repeated iteratively until each region becomes a built region. The resulting divisions are based on a random generated number that considers a given building region midpoint and the global building seed. A given region will always produce the same random number, which ensures that the division process will occur exactly in the same conditions in each required generation. The authors also implemented some architectonic rules to ensure the proper generation of the final 
geometry. Figure 2.10 presents different division stages affecting the same buildings using a lazy strategy, as it is called by the authors.

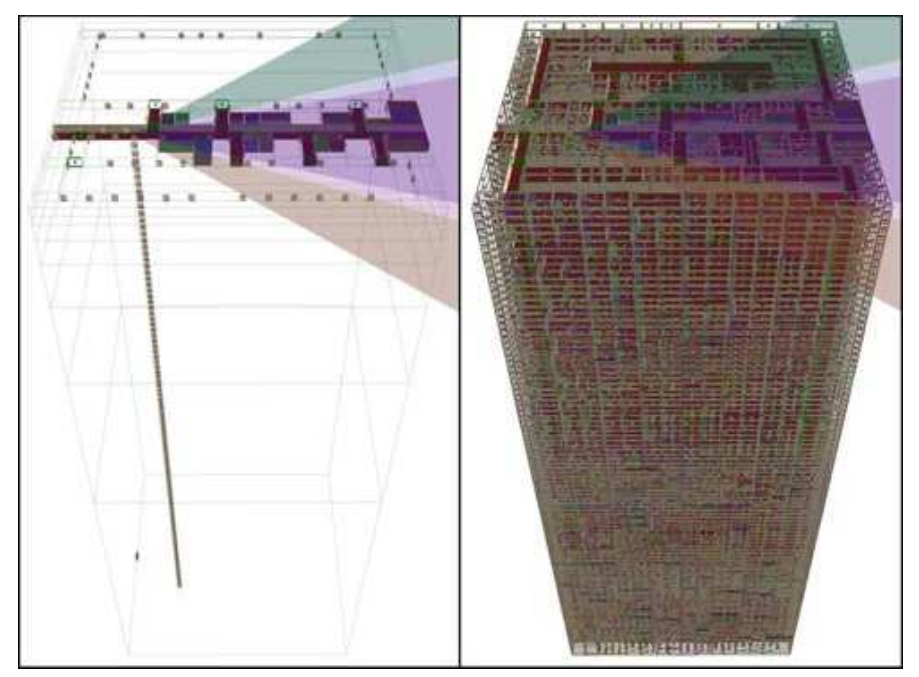

Figure 2.10 - Building with floors produced in real-time through the lazy approach proposed by Hahn et al. (2006): on the image's left side an incomplete building is presented. On the right side, there is the same building completely generated.

\subsubsection{Interior rooms generated through Voronoi diagrams and constrained by convex layouts}

Rinde (2008) developed an algorithm that generates rooms inside convex polygons representing building limits (Figure 2.11). It receives a set of specifications for building generation such as constraint walls, windows and doors and also a couple of parameters defining region types and room types. Then, it mounts the building skeleton with a mandatory corridor for layouts with large dimensions and creates regions for grouping sub-regions or final rooms. These last elements are generated using a weighted Voronoi diagram that spreads rooms inside regions considering the desired room weights. Meanwhile, a room graph is created in order to connect rooms and then, the room types are defined accordingly with the input parameters.

This approach generates traversable buildings considering convex shapes as constraints. However, some issues were identified: the impossibility of managing the 


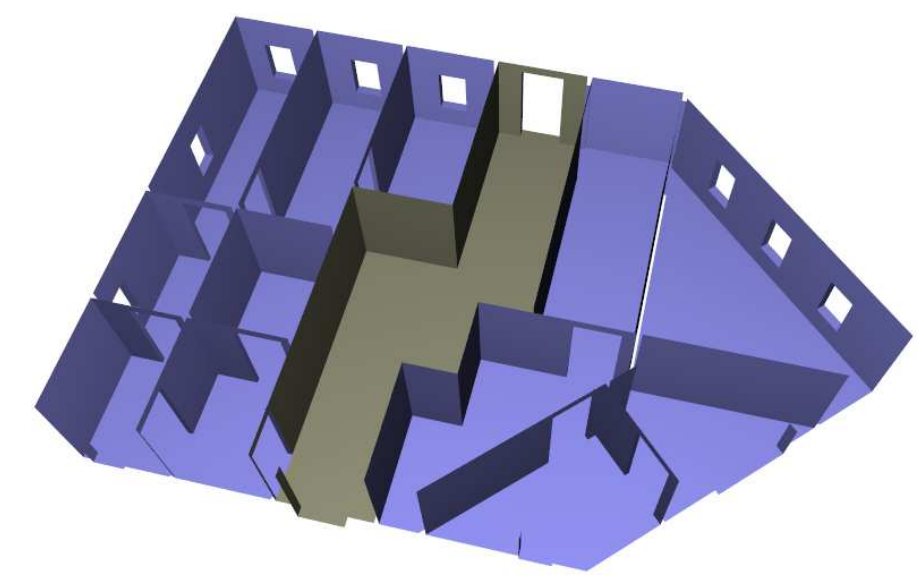

Figure 2.11 - Rinde (2008) used a Voronoi diagram to subdivide an irregular polygon into rooms.

number and size of rooms to be generated; the confinement to the generation of structures disregarding geometric holes; finally, the absence of visual details such as textures.

\subsubsection{Rule-based generation/reconstruction of buildings}

Rodrigues et al. (2008b,a) developed a multifaceted method capable of generating portuguese houses and reconstructing ancient roman houses, both traversable. A set of rules is properly applied, accordingly with each case, to respect architectonic requirements. To generate portuguese houses, the portuguese regulation for urban buildings (RGEU, 2008) was adopted. The rules of Vitruvius, referenced by Maciel (2006), are used to regulate the process of roman houses reconstruction. The virtual building models generation process starts with the definition of a room graph, considering the referred rules. Then, the set of shapes that represent rooms is distributed in a certain area, to form the floor plan. The last step is the 3D model generation in which the extrusion of the walls containing the required doors and windows and also the placement of the roof occurs. Rodrigues et al. (2008c) revealed, in detail, the methods and processes included in the generation of roman houses. Their system starts the generation process considering user rules - provided through a generation grammar - or spontaneous L-system. Both provide a list of rooms. 
Those rooms are then mapped into a multi-layer graph defining containers and also room connections. Next, a floor plan is produced through dimensioning operations and container-based arrangements. In the final step, walls are extruded - ensuring transitivity between connected rooms - and floors, ceilings and roofs are properly created. Later, Rodrigues et al. (2010) extended their work to provide virtual building models in several formats (for example, X3D and VRML). The improvement foresees the integration with virtual worlds like Second Life. The aforementioned works culminated on a Phd thesis (Rodrigues, 2010) that has some images depicting virtual reconstructions of roman houses like the ones that can be seen in Figure 2.12 .

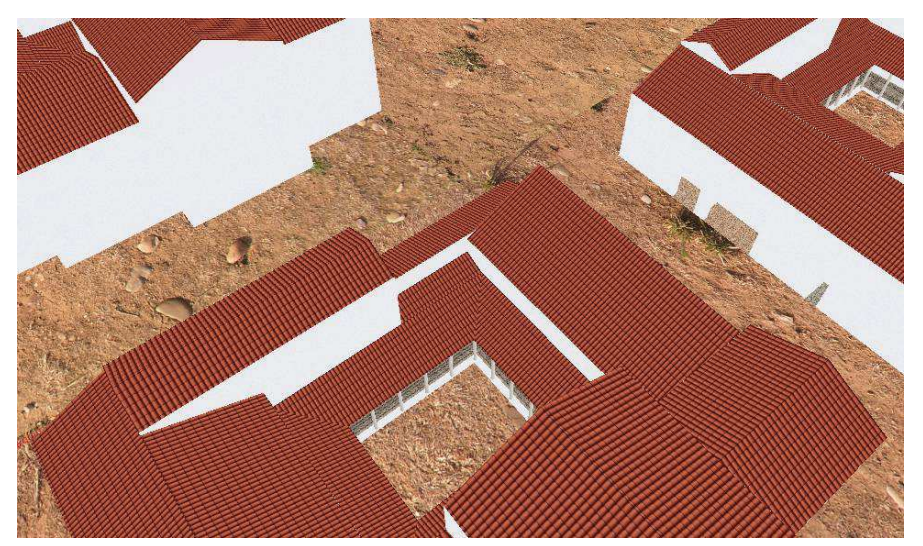

Figure 2.12 - Virtual environment depicting roman houses from Rodrigues (2010). A set of reconstruction rules is used to guide the cultural heritage recovering process from the floor plan stage to the complete 3D building model.

\subsubsection{Squarified treemaps for virtual buildings generation}

The squarified treemap (Bruls et al., 1999) is a subdivision strategy (Figure 2.13) adapted for the generation of buildings with interiors by a few authors (Marson and Musse, 2010; Mirahmadi and Shami, 2012). The strategy consists in splitting rectangular areas considering a set of weights and the following key rule: in each division it must be ensured that the aspect ratio has the closest value to 1. Marson and Musse (2010) applied it in two phases. The method starts by dividing the building square in three distinct functional areas: social, private and service. Then, the squarified 
treemap is reapplied to subdivide the referred zones into rooms, accordingly with the user requirements. Rooms are connected taking into account the connection rules imposed by authors. The final step is the placement of a corridor to connect the unreachable rooms. Mirahmadi and Shami (2012) used the same method for splitting rectangular areas, with some optimizations at the corridor placement step, to increase the realism of the architectural designs.

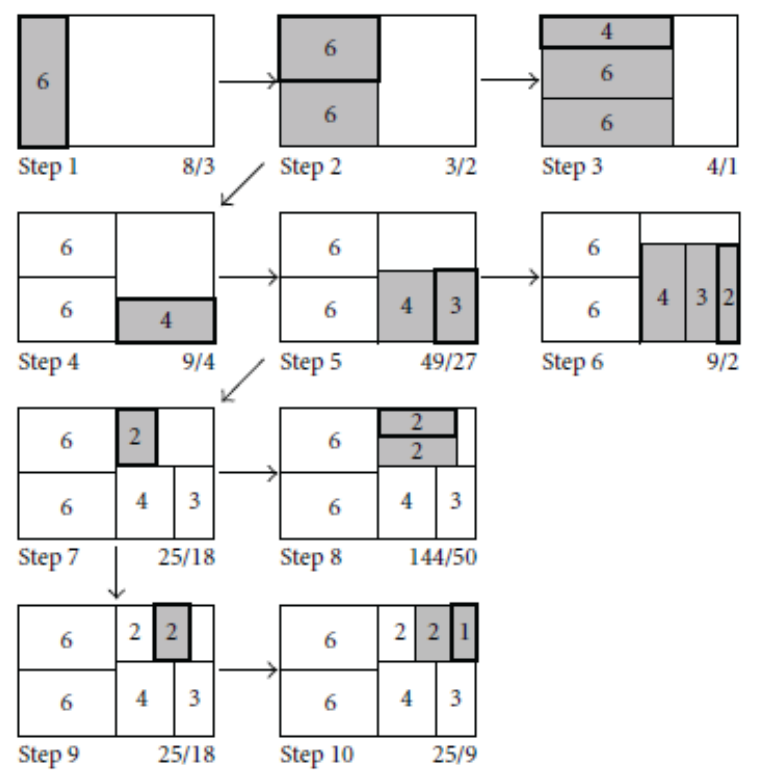

Figure 2.13 - Squarified treemaps operation (Bruls et al., 1999). Considering the following sequence of rectangular areas $6,6,4,3,2,2$, and 1 , a descendant sorting algorithm optimizes the process which tries to arrange the areas inside a rectangular container, in order to find the aspect ratio with the closest value to 1 , in each iteration.

\subsubsection{Residential buildings generation based on bayesian net- works}

An approach for generating virtual models of residential buildings, focusing the production of floor plans highly based on architectonic knowledge, was presented by Merrell et al. (2010). In this approach, a set of high-level requirements must be provided by an user and then expanded into an architectural program, that consists in a bubble diagram providing a list of rooms, respective sizes and connections. The 
architectural program - compatible with the referred requirements - is provided by a bayesian network that contains 120 of these structures manually encoded. Next, the floor plan is determined through stochastic optimization that occurs using operations such as wall sliding and room swapping. Afterwards, the 3D model generation takes place. A template is then selected, to obtain a building garnished with a style that defines the appearance of doors, windows, roof and other elements. Considering the selected style, walls are raised, windows and doors are placed and roofs are also created to cover the building. Internally, a staircase is properly placed to provide a connection between floors at different levels. The distinct phases of this process are depicted in Figure 2.14.
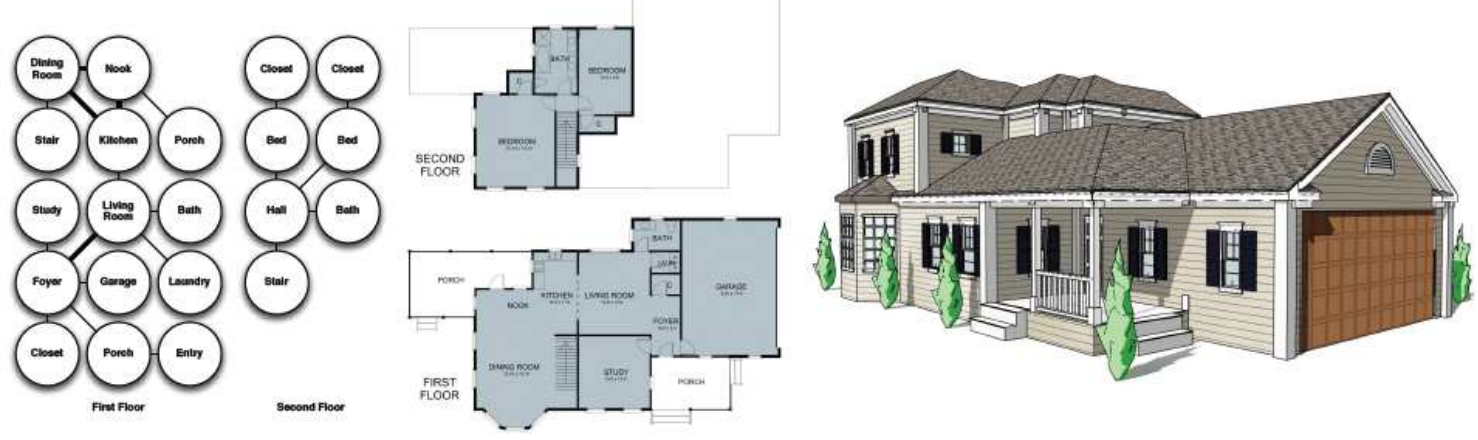

Figure 2.14 - The process of house generation based on bayesian networks, trained with real data (Merrell et al., 2010): the left image depicts a room graph extracted from the bayesian network; the central image reveals a couple of floor plans adequately rearranged by the system; finally, the right image presents the resulting 3D house model, after the application of the generation process.

\subsubsection{Grid approach focusing floor plan generation}

A method inspired in geometric grids used by architects to aid the manual drawing of floor plans was proposed by Lopes et al. (2010), who developed a grid-based algorithm to allow the placement and expansion of rooms. The algorithm expects a few inputs to start the floor plan subdivision process, namely a building footprint polygon. Additionally, the user can establish some constraints: grid dimension, list of rooms with respective type (public, private, hallway), room dimensions, adjacency 
constraints (room arrangement defining, for example, that a kitchen should be next to the living room) and also connectivity requirements (transitivity between rooms). Considering these constraints, the subdivision of the floor plan occurs hierarchically. Firstly, the functional zones are determined (e.g. public and private zones) as it was also suggested by Marson and Musse (2010). Then, rooms are placed in the proper zones and expanded using the grid approach. The placement step defines the appropriated position of a room in the grid. Then, growth methods based on cells filling are applied to make the rooms expand through the available area. At last, the connections are processed to complete the floor plan: doors are placed to satisfy the explicit connectivity requirements and then, the rest of the rooms are properly connected accordingly, with rules established in this approach.

\subsubsection{Generative Modelling Language (GML) for the pro- duction on virtual buildings}

GML - acronym for generative modelling language in the specific context of this work (different from Geographic Modelling Language) - is an imperative programming language used to define geometric structures based on split grammars (Hohmann et al., 2010), that also supports the generation of building interiors. The available operations include the creation, modification and termination of scopes and also relative and absolute subdivisions. The effectiveness of the tool was demonstrated through a case-study that consisted in the reconstruction of the University of Technology in Graz, Austria. The modelling process starts of by identifying superstructures that are subparts of the same building that resulted from the analysis to the available floor plan documents. Each superstructure is subdivided into smaller vertical parts which are then splitted by floors and rooms. The connectivity between building elements is properly ensured accordingly to each case: floors are linked through staircases, rooms can be reached through the modelled corridors and superstructures communicate using bridges. More information about this language can be found in Havemann (2005). 


\subsubsection{Component-based modelling of virtual buildings}

Leblanc et al. (2011) proposed a tool based on programming that supports a virtual building component-oriented modelling. Each component can be seen as a geometric element of the building (2D or 3D shape), composed by faces or regions. The referred programming tool allows some operations upon components such as attribute alteration (add, modify or delete), component connection (consists on linking a component coordinates system to another component region) or creation (that includes, for example, instantiating, slicing, splitting, extruding or roofing components for geometric transformations or decomposition). Despite the high-level of freedom, authors suggests that a sequence of steps should be considered in order to obtain the expected virtual model. Step one is space partitioning, that starts with a face to hold building components. The starting face will be subjected to several operations, such as slicing and splitting, to obtain the definitions for roofs, storeys (floors) and also rooms (categorized as living room, kitchen, bedroom, bathroom, etc.) inside each storey. The second step provides simple 3D geometry-based extrusion operations regarding rooms and also building exterior components, such as simple external facades. Step three is concerned with architectural elements: placement of frames windows, doors and other special components, such as balconies. The placement of doors and windows requires special operations upon room components, specifically subtractions, to create holes in room walls. At last, the proper placement of furniture in the building regarding some rules - for example, not placing light fixtures too close from walls - takes place. Despite the flexibility of the proposed tool, some issues were exposed: designers must have programming skills and despite the automation level of the tool, a virtual building can take a few hours to be produced from the scratch, which can be a considerable amount of time.

\subsubsection{Producing virtual 3D buildings from pre-designed floor plans}

A tool for the expeditious production of 3D virtual buildings, including interiors and outer facades, considering scanned floor plans among other input informations 
such as photos, room areas, location and surroundings was proposed by Santos et al. (2011). The first operation to be performed is the floor plan vectorization. Thus, considering the submitted floor plans, the tool starts by automatically detecting the scale considering the calculations made through the scale value - obtained from Optical Character Recognition (OCR) - in conjunction with pixel distance measurements. Then, the user must contour the outline of each floor plan to convert the raster image into vector. This task is assisted by a snapping pixel function to accelerate the process and reduce human error. In this stage, an automatic process attempts to detect doors and windows, which are then referenced by the user, in order to overcome the lacks of the automatic detection process. Besides this, the user is also responsible for indicating staircases - used to connect floors at different levels - and placing furniture. The authors have defined some rules for furniture placement. For example, there are constraints to ensure that objects only appear in the proper rooms and rules to delete objects that do not respect a minimum distance relatively to others. The exterior environment concerning the virtual building surroundings is also produced from $2 \mathrm{D}$ polygons, reserved for plant areas with trees, roads, etc. The entire geometry providing $3 \mathrm{D}$ visualization is produced considering the following steps: wall extrusion, placement of doors and windows, inclusion of interior furniture and exterior objects and finally roofs creation. Moreover the textures and colors applied to building elements such as walls, ground and ceilings can be extracted from user images - using computer vision techniques - to improve the 3D model realism. One final remark goes to the creation of visitations which can be defined by an user or automatically mounted by that tool. Figure 2.15 depicts the aspect of some generated virtual buildings.

The prototype Building Model Generator (BMG) was another proposal that appeared even earlier than the previously mentioned (Lewis and Séquin, 1998). The prototype receives as input 2D floor plans, previously developed in a commercial CAD software. Then, the floor plans are properly converted to a compatible BMG format and the prototype posteriorly detects and corrects small geometrical inconsistencies. Those floor plans are analysed in order to extract rooms and portals. Further steps include the extrusion of walls and also the proper placement of doors 

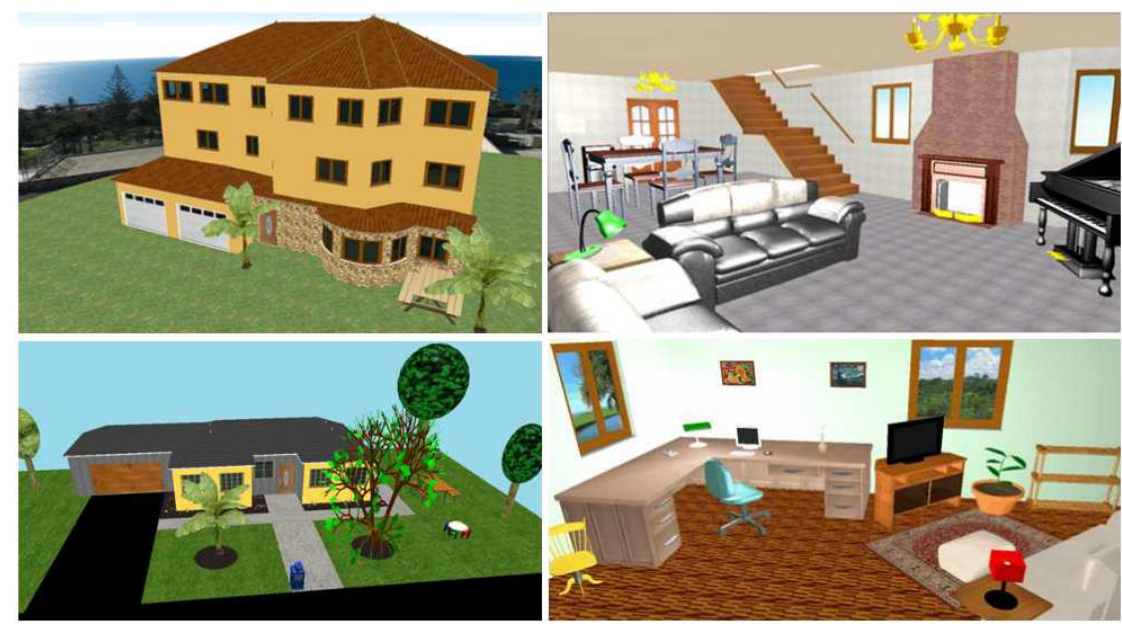

Figure 2.15 - Virtual environments produced using the tool developed by Santos et al. (2011). On the left, there is a couple of images depicting the generation of exteriors, including building facades and surroundings. On the right the results regarding the generation of building interiors is presented.

and windows. An interactive editor is provided to allow some adjustments on building elements, including materials. Finally, there is a complementary tool - a staircase generator - that enables the proper placement of staircases. The final result is a virtual 3D model of a building with connected floors.

\subsubsection{Ontology-based generation of traversable buildings}

The ontology-based modelling of traversable buildings was also addressed by a few authors. The current subsection will expose each work.

\section{Virtual world grammar for automatic generation of Virtual Worlds}

A virtual world (VW) generator focused on institutions was proposed by Trescak et al. (2010). The system requires the specification of the evolved activities. An ontology catalogs these activities and also shape grammar properties. The role of the shape grammar is similar to the observed in aforementioned works: defining an object geometry. Heuristics and validations ensure a regulated generation of the entity 
required for representation. The case study provided by the authors is an auction system. To represent the institution, a set of activities - also known as scenes and later mapped as buildings or inner rooms - must be provided: admission, item registration, auction, auction info and also entrances and exits. Then, a VW Grammar is constructed, based on an ontology. The referred ontology comprises both activities and the shape grammar. An object mapping is also performed in order to relate ontological objects with the proper shape grammar responsible for its representation in the virtual world. After the completion of VW Grammar, a set of heuristics, validations and evaluations regulate the generation of the institution, that is made in two main steps: floor plan production and 3D virtual model transformation. Figure 2.16 depicts the described method.

\section{Framework of procedural techniques}

Tutenel et al. (2011) proposed an inclusive framework that produces virtual buildings using several procedural techniques. Building floor plan definitions must be provided using declarative language instructions, that trigger a sequence of steps including the loading of the floor plans and the selection of the techniques that should be used in each phase of generation. For example, lot (3D building gross model) generation can be made through CGA shape grammar and the floor plan might be produced using a grid approach like the one presented by Lopes et al. (2010). Some regulation mechanisms are applied to guide the whole process. One of the most important is the semantic moderator that shares relevant building informations with the wrapper of the procedural component, that is capable of assigning a convenient procedural technique to each building element. Also, the use of a semantic model based on a building ontology - in combination with the informations shared by the semantic moderator, provides regulation to avoid the following types of conflicts: intersection conflicts (e.g. nonsense overlaps between objects), functional conflicts (bad placement of objects, such as regular room windows in bathrooms) and exclusion conflicts (e.g. impracticable use of objects by misplacing or inaccessibility). According to the authors, the lack of backtracking on some techniques might constitute a weak point regarding the emergence of inconsistencies. 


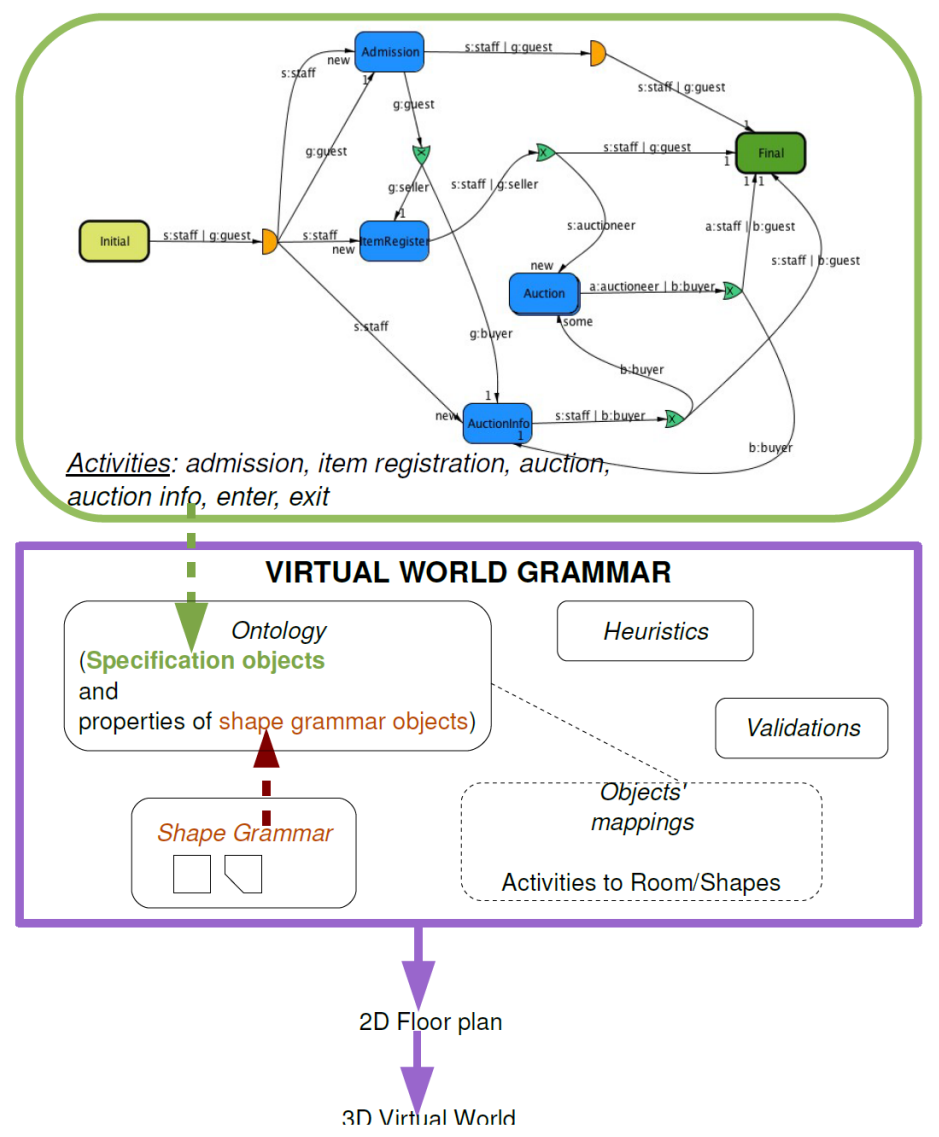

Figure 2.16 - Virtual World generator process proposed by Trescak et al. (2010) and exemplified through an auction system. The specification of the activities establishes the structure of the institution. These activities, in combination with the shape grammar, form the ontology. Ontology objects are mapped in order to establish a relation with a shape grammar for geometric representations purposes. Validations, heuristics and also evaluations regulate the process to obtain the institution $3 \mathrm{D}$ virtual model.

\subsection{Summary}

This chapter started by presenting the versatility of ontologies applied in a wide variety of solutions incorporating, 3D virtual models to serve areas such as medicine, industry, cultural heritage and design. Then, standard knowledge-based representations for virtual urban environments were addressed: CityGML was referred and shown along with the building definition proposed by this standard. Moreover, the 
procedural modelling solutions were extensively documented, including the generation/reconstruction of virtual urban environments and traversable buildings. Each topic had a few works that also consider ontologies. Regarding the traversable buildings, table 2.1 presents a brief summary of the most relevant approaches, comparing main procedural techniques, modelling purposes, resulting geometry types and use of ontologies.

Table 2.1 - Summary of the procedural modelling works, regarding buildings with interiors. The following aspects are compared for each work: main procedural techniques, modelling purposes, resulting geometry types and use of ontologies.

\begin{tabular}{cccc}
\hline Work & $\begin{array}{c}\text { Procedural } \\
\text { Approach }\end{array}$ & Geometry & $\begin{array}{c}\text { Ontology or } \\
\text { Semantics }\end{array}$ \\
\hline $\begin{array}{c}\text { LaHave House } \\
\text { architectural } \\
\text { service }\end{array}$ & Shape Grammar & Aggregation of & Not specified \\
(Rau-Chaplin & rules & rectangular rooms & \\
et al., 1996) & Room graph & & \\
\hline Martin's House & generation, & Aggregation of & Not specified \\
Generator & placement and & rectangular rooms & \\
(Martin, 2005) & expansion & & Not specified \\
\hline $\begin{array}{c}\text { CSG-based } \\
\text { virtual buildings } \\
\text { generation }\end{array}$ & 2D and 3D CSG & Aggregation of & \\
(Bradley, 2005) & algorithms & rectangular rooms & \\
\hline $\begin{array}{c}\text { Lazy generation } \\
\text { of virtual }\end{array}$ & Real-time & & Not specified \\
buildings (Hahn & subdivision of & Rectangles & \\
\hline fl al., 2006) & floors & & \\
\hline
\end{tabular}


Table 2.1 - continued from previous page

\begin{tabular}{|c|c|c|c|}
\hline Work & $\begin{array}{l}\text { Procedural } \\
\text { Approach }\end{array}$ & Geometry & $\begin{array}{c}\text { Ontology or } \\
\text { Semantics }\end{array}$ \\
\hline $\begin{array}{l}\text { Automatic } \\
\text { building modeling } \\
\text { based on Voronoi } \\
\text { (Rinde, 2008) }\end{array}$ & $\begin{array}{l}\text { Subdivision of } \\
\text { convex layouts } \\
\text { using Voronoi } \\
\text { diagrams }\end{array}$ & $\begin{array}{c}\text { Convex shapes for } \\
\text { exterior } \\
\text { constraints; } \\
\text { Interiors formed } \\
\text { by inner walls } \\
\text { random } \\
\text { arrangement }\end{array}$ & Not specified \\
\hline $\begin{array}{c}\text { Rule-based } \\
\text { generation of } \\
\text { architectural- } \\
\text { period houses } \\
\text { (Rodrigues, 2010) }\end{array}$ & $\begin{array}{c}\text { Floor plans } \\
\text { generated through } \\
\text { multi-layer graph } \\
\text { and geometric } \\
\text { solver; } \\
\text { Incorporation of } \\
\text { legal and } \\
\text { architectonic rules }\end{array}$ & $\begin{array}{l}\text { Aggregation of } \\
\text { rectangular rooms }\end{array}$ & Not specified \\
\hline
\end{tabular}

Generation of

virtual buildings

based on

Squarified

Division of

squarified

Treemaps

treemaps (Marson

(weighted areas

rectangular

and Musse, 2010;

tree)

footprints into

Not specified

Mirahmadi and

Shami, 2012) 
Table 2.1 - continued from previous page

\begin{tabular}{|c|c|c|c|}
\hline Work & $\begin{array}{l}\text { Procedural } \\
\text { Approach }\end{array}$ & Geometry & $\begin{array}{l}\text { Ontology or } \\
\text { Semantics }\end{array}$ \\
\hline $\begin{array}{l}\text { Generation of real } \\
\text { data-based } \\
\text { residential } \\
\text { buildings (Merrell } \\
\text { et al., 2010) }\end{array}$ & $\begin{array}{c}\text { Stochastic } \\
\text { optimization of } \\
\text { floor plans } \\
\text { provided by } \\
\text { bayesian networks } \\
\text { trained with real } \\
\text { data }\end{array}$ & $\begin{array}{l}\text { Aggregation of } \\
\text { rectangular rooms } \\
\text { with slidable walls }\end{array}$ & $\begin{array}{c}\text { Use of semantics } \\
\text { (no details) }\end{array}$ \\
\hline $\begin{array}{c}\text { Grid-based } \\
\text { generation of } \\
\text { buildings' floor } \\
\text { plans (Lopes } \\
\text { et al., 2010) }\end{array}$ & $\begin{array}{l}\text { Grid and } \\
\text { algorithms for } \\
\text { room's placement } \\
\text { and expansion }\end{array}$ & $\begin{array}{l}\text { Aggregation of } \\
\text { rectangular rooms }\end{array}$ & Not specified \\
\hline $\begin{array}{c}\text { Production of } \\
\text { virtual buildings } \\
\text { with GML } \\
\text { (Hohmann et al., } \\
2010 \text { ) }\end{array}$ & $\begin{array}{c}\text { Imperative } \\
\text { programming } \\
\text { language oriented } \\
\text { to geometric } \\
\text { aspects }\end{array}$ & Rectangles & $\begin{array}{l}\text { Semantics to } \\
\text { control scopes of } \\
\text { functions }\end{array}$ \\
\hline $\begin{array}{l}\text { Component-based } \\
\text { modeling of } \\
\text { virtual buildings } \\
\text { (Leblanc et al., } \\
\text { 2011) }\end{array}$ & $\begin{array}{l}\text { 2D an 3D tool } \\
\text { based on CSG } \\
\text { and split } \\
\text { grammars }\end{array}$ & Mainly rectangles & $\begin{array}{l}\text { Semantics for } \\
\text { building } \\
\text { components }\end{array}$ \\
\hline
\end{tabular}


Table 2.1 - continued from previous page

\begin{tabular}{|c|c|c|c|}
\hline Work & $\begin{array}{l}\text { Procedural } \\
\text { Approach }\end{array}$ & Geometry & $\begin{array}{l}\text { Ontology or } \\
\text { Semantics }\end{array}$ \\
\hline $\begin{array}{l}\text { Production of 3D } \\
\text { buildings from } \\
\text { pre-designed floor } \\
\text { plans (Santos } \\
\text { et al., 2011; Lewis } \\
\text { and Séquin, 1998) }\end{array}$ & $\begin{array}{c}\text { Methodology for } \\
\text { generating 3D } \\
\text { buildings, } \\
\text { considering the } \\
\text { existing floor } \\
\text { plans }\end{array}$ & $\begin{array}{l}\text { Shapes retrieved } \\
\text { from scanned or } \\
\text { CAD floor plans, } \\
\text { mainly rectangles }\end{array}$ & $\begin{array}{c}\text { Custom } \\
\text { established } \\
\text { semantics (Santos } \\
\text { et al., 2011); } \\
\text { attached semantic } \\
\text { information } \\
\text { (Lewis and } \\
\text { Séquin, 1998) }\end{array}$ \\
\hline $\begin{array}{l}\text { Virtual world } \\
\text { generator focused } \\
\text { on institutions } \\
\text { (Trescak et al., } \\
2010 \text { ) }\end{array}$ & $\begin{array}{l}\text { Combination of } \\
\text { ontologies with } \\
\text { shape grammars } \\
\text { and control } \\
\text { heuristics and } \\
\text { validations }\end{array}$ & Rectangles & $\begin{array}{l}\text { Ontology for } \\
\text { cataloguing } \\
\text { institution } \\
\text { activities }\end{array}$ \\
\hline $\begin{array}{c}\text { Framework } \\
\text { integrating } \\
\text { Procedural } \\
\text { Techniques } \\
\text { (Tutenel et al., } \\
\text { 2011) }\end{array}$ & $\begin{array}{c}\text { Application of } \\
\text { different } \\
\text { procedural } \\
\text { techniques to } \\
\text { generate different } \\
\text { building } \\
\text { components }\end{array}$ & Rectangles & $\begin{array}{l}\text { Building ontology } \\
\text { (semantic model) }\end{array}$ \\
\hline
\end{tabular}

Summing up, there is a wide variety of procedural modelling works addressing the generation of virtual buildings' interiors with distinct approaches: tools based on shape grammars (Rau-Chaplin et al., 1996), CSG algorithms (Bradley, 2005), expanding rooms (Martin, 2006), grid-based approaches (Lopes et al., 2010), squarified 
treemaps (Marson and Musse, 2010; Mirahmadi and Shami, 2012), rule-based solutions (Rodrigues, 2010) with architectonic awareness (RGEU, 2008; Maciel, 2006), generative modelling languages (Hohmann et al., 2010; Leblanc et al., 2011) and others. However, most of them only deal with rectangular shapes or geometries uniquely formed by right angles. Alternatively, (Rinde, 2008) addressed the production of virtual buildings composed by convex shapes with a Voronoi diagram approach. However, some issues remain to be addressed, namely floor plan holes or concave constraints are not supported and rooms generation is hard to manage.

Considering the referred issues, a novel procedural modelling methodology will be proposed along with a building ontology - for regulation purposes - inspired in the exposed ontology works and in the CityGML standard. This proposal contains a process that relies in some of the aforementioned procedural modelling solutions, specifically in the floor plan generation and the 3D transformation. The refinements made during the development of this methodology will be progressively presented until the final solution, that supports the generation of buildings constrained by arbitrary shapes, with rooms limited by a configurable number of inner walls. The proposed solution also intends to enable the generation of virtual buildings in different architectural contexts, taking advantage from the integrated building ontology. General purpose buildings belonging to the generic ontology and buildings based on roman architecture - specifically domus - are addressed both as case studies and for demonstration purposes. Moreover, a stochastic approach regarding the random generation of virtual generic buildings will be presented as a way of automating the virtual models production using the proposed methodology. 

This chapter starts by exposing the current issues in Procedural Modelling along with the justification of some strategic decisions made during the development of this work. The remainder of the chapter generically addresses the proposed procedural modelling methodology for the production of buildings composed by arbitrary shapes, as well as its supporting regulation structure, that is the buildings' ontology (Adão et al., 2012).

\subsection{Problem Definition and General Proposed Frame- work}

Regardless of the variety of approaches that can be found in the vast procedural modelling bibliography, techniques capable of joining traversable buildings and non-squared geometries in the same bundle are still scarce. Moreover, mechanisms regarding extensions to specific architectonic styles seem to be under-explored. The identification of such shortcomings triggered the definition of a new ontology-based methodology, capable of generate buildings constrained by arbitrary shapes and composed by inner rooms, that can also vary in terms of geometry. 
At the beginning of this work, the possibility to produce different types of buildings with possibly diverse architectonic styles was pursued. Ontologies were then chosen to overcome the challenge, because for procedural modelling, ontologies may act as regulatory structures, that relate a building's conceptual parts with the geometrical aspects considering both as distinct objects. Moreover, they promote scalability and re-usability, as it was demonstrated by Béhé et al. (2014); Mignard and Nicolle (2014), providing useful extension mechanisms, as it commonly happens on information systems' data models. There was also some experience gathered in the combination of procedural modelling and ontologies, as it was previously explored, with success, in the Expedite Virtual Reconstruction of Cultural Heritage Sites (ERAS) research project ${ }^{1}$, in which a semi-automatic methodology was applied for the virtual reconstruction of virtual buildings belonging to distinct epochs, using previously extracted rules from textual descriptions. The previously mentioned characteristics and advantages of ontologies, together with the experience acquired from the ERAS project, led to the planning and design of a generic ontology that can be extended to characterize different kinds of building in different architectural contexts. In this work, the roman architectural style is the first case-study presented mainly because there is a lot of accurate documentation available that can be used to support and validate some of the virtual models resulting from the proposed procedural modelling methodology (Dylla et al., 2009; Rodrigues et al., 2008b,a; Maciel, 2006). Simultaneously, the viability of adapting ontology extensions to the proposed methodology, aiming the generation of different structures was also being ascertained. These extensions are made from a generic ontology which was designed considering CityGML (Gröger et al., 2008, 2012): a standard that can be seen as a set of recommendations for describing virtual urban environments.

The procedural modelling methodology was addressed soon after the ontology design phase. During the literature review, it became obvious that most of the existing procedural modelling methods for the generation of virtual buildings populated with inner rooms, starts by solving the floor plan production task. The identification of

\footnotetext{
${ }^{1}$ The project "Expedite Virtual Reconstruction of Cultural Heritage Sites" (ERAS PTDC/EIA-EIA/114868/2009) aimed to create a tool for the field of archaeology which made it possible to rebuild and visualize georeferenced archaeological sites with complete buildings, indoors and outdoors.
} 
this pattern led to the first challenge: to find an adequate space division approach. An extensive research took place and a wide variety of approaches was analysed. Most of them rely on the rectangular subdivision or aggregation of rectangular shapes. Rau-Chaplin et al. (1996) created a shape grammar-based tool to allow the collaborative building plan and design between architects and clients. According to the authors, tiles are room's configurations related with furniture organization among others - that suggest that rooms are confined to rectangular shapes: a guess reinforced by the floor plans depicted on their paper. Martin (2005) proposed a Monte-Carlo expansion approach that makes the rooms growth and fill a given footprint. He confirmed that room's base shapes are also confined to rectangles as other works proposed by Lopes et al. (2010), (Rodrigues, 2010), Merrell et al. (2010) or Tutenel et al. (2011). An alternative approach (Marson and Musse, 2010; Mirahmadi and Shami, 2012), operating with same kinds of shapes, involve the floor plan subdivision with squarified treemaps Bruls et al. (1999). This solution focus the space provisioning and considers a set of rectangles - sorted by weights of occupation along with their aspect ratios to arrange rooms inside a given rectangular container. The goal is to fit this rectangles inside the container forcing them to have the closest possible aspect ratio from value 1 , in each iteration. Such restrictive method is inappropriate to generate buildings in scenarios that require a certain level of controllability over the placement of rooms (for example, in tasks regarding building's reconstruction or digital preservation). The same issue affects the lazy approach of Hahn et al. (2006) who proposed a rectangular subdivision of building floors based on random number generation, in real time. Others (Hohmann et al. (2010),Leblanc et al. (2011)) opted by generative modelling languages and CSG algorithms that require designers to have programming skills. Besides, virtual buildings' construction process seems to be quite tedious, despite the announced production time decreasing tendency, over the iterations with the tools. Only one work was found with the goal of dealing with non-rectangular shapes during the floor plan production: Rinde (2008). He developed a Voronoi-based method to subdivide randomly shaped rooms constrained by convex polygons. This work does not address the geometric holes or even concave constraints. Besides, the number of rooms to be generated seems hard to manage since they are created based on their size and constraint polygon's 
available space.

Alternatively, treemap (Johnson and Shneiderman, 1991) was identified as an effective space partition approach (specially when using recursion) simple to apply, highly controllable, predictable and quite flexible. Although this approach have been applied to the rectangular space partitioning, the resulting rectangles can be interpreted as bounding boxes defining room's shapes prone to modification through the insertion or remotion of shape's edges that represent wall's segments. Thereby, treemap approach is the chosen candidate to integrate the proposed procedural modelling methodology, specifically in the floor plan generation task.

The treemap approach was initially applied to generate roman houses - also known as domus - composed by rectangular divisions and rectangular layouts, as it will be seen in chapter 4 . Buildings with such room configurations are suitable for the use of this approach. Although the treemap approach is usually associated with squared shapes, this did not discourage its use in this work and, in fact, it was properly adapted to deal with other shapes in chapter 5 . The adaptations made to the original treemap algorithm enabled the subdivision of constraint convex shapes into smaller rectangular-based compartments, moulded by the referred constraint shapes. Some results are provided with the virtual reconstruction of real and fictitious ruins. In chapter 6, the treemap approach is enhanced again to support the generation of buildings constrained by non-convex layouts, through the implementation of a technique that relies in a labelling approach used by Binary Space Partitioning (BSP) trees to define disposable parts, for further suppression. In this proposal, BSP labelling approach was adapted to indicate holes in the floor plan, thus being renamed to fake-concave. Also in chapter 6, a process for defining the shapes of inner rooms is proposed which consists in adding or removing constraint room walls to define a room shape.

After the space division step, the remaining floor plan is achieved in a process that is similar in every iteration of the subdivision approach: interior/exterior doors and windows are placed and 2D's expanded to occupy a certain wall portion. Finally, the $3 \mathrm{D}$ virtual model is produced through a set of extrusions, roof placement and detail completions, as it will be seen in this document. 
The next sections will introduce the proposed procedural modelling methodology, along with the supporting ontology.

\subsection{Ontology for Buildings}

This section addresses the buildings' ontology that is the first regulatory structure for the proposed modelling methodology, by establishing an organization concerning buildings and its integrating elements, from a generic viewpoint. Accordingly to the ontology, a building is composed by temporary building part containers (for the arrangement of other building parts) or final building parts; each building part contains at least one floor; each floor is composed by divisions that can be temporary containers for room arrangements or final rooms. Transitions, such as doors and windows, can be related with floors or/and divisions. The buildings' ontology is partially based on the CityGML standard (Gröger et al., 2008, 2012) and provides the possibility of defining a sequential set of operations and also geometric transformations in agreement with each element, maintaining the coherence of the virtual model and generation control. The proposed ontology was also extended to be used in the regulation of roman houses generation. A few more structures were added to support some particularities observed in the roman architecture such as columns, pools (impluviums) and roof openings (compluviums). Both of these ontological structures - generic and roman - will be explained next.

\subsubsection{Generic ontology}

The buildings' generic ontology was defined considering an abstraction effort to identify and connect the architectonic elements that can be found in a building, regardless of era or style. Thus, it is defined that a Building structure is the root container that holds BuildingPart instances, that can be seen as horizontal compositions (parts of the same building distributed at the ground level). From this viewpoint, each tower belonging to a castle would be considered as a castle's building part, for example. In turn, each BuildingPart instance can have inner building 
parts for arrangement purposes or can aggregate one or more Floor structures which can be defined as the vertical parts of each building part. In other words, as a floor inherits from BuildingPart, floors can be seen as vertical building parts that rise inside a given building part. Each Floor structure contains a set of Division structures that can be rooms or temporary compartments for arrangement purposes. Divisions are typically connected to other divisions or to the exterior by transitions (equivalent to Transition structures), as for example doors and windows. Transitions share overlapping boundary surfaces (equivalent to BoundarySurface structures), belonging to different divisions or floors. These BoundarySurface structures represent the limits of a floor or a division, that can extend to WallSurface, CeilingSurface or GroundSurface structures. AbstractElement is useful to describe special architectonic features, such as columns, footers, portals or even furniture. Lastly, the Roof represents the cover of each building part, usually placed in the top of the last floor. Figure 3.1 depicts the generic ontology schema.

To promote the reuse of features, specifically of the geometry and appearance, it was decided that each ontological element extends from the same father: Worldobject. Figure 3.2 illustrates the referred inheritance.

This ontology is a generic skeleton that integrates a set of empty definition classes to keep it structurally open to concrete adaptations to other supports, as for example, data models or ontology-based grammars.

\subsubsection{Generic ontology elements}

The proposed ontology contains a set of interrelated classes. This subsection will provide a brief description of each one of them:

- WorldObject: this is the base class for each ontological element. WorldObject contains a Geometry and an Appearance, which define an object shape and look, respectively.

- Block: represents the Building holding area. In other words, it can be seen as the exterior ground frame that will support Building structures; 


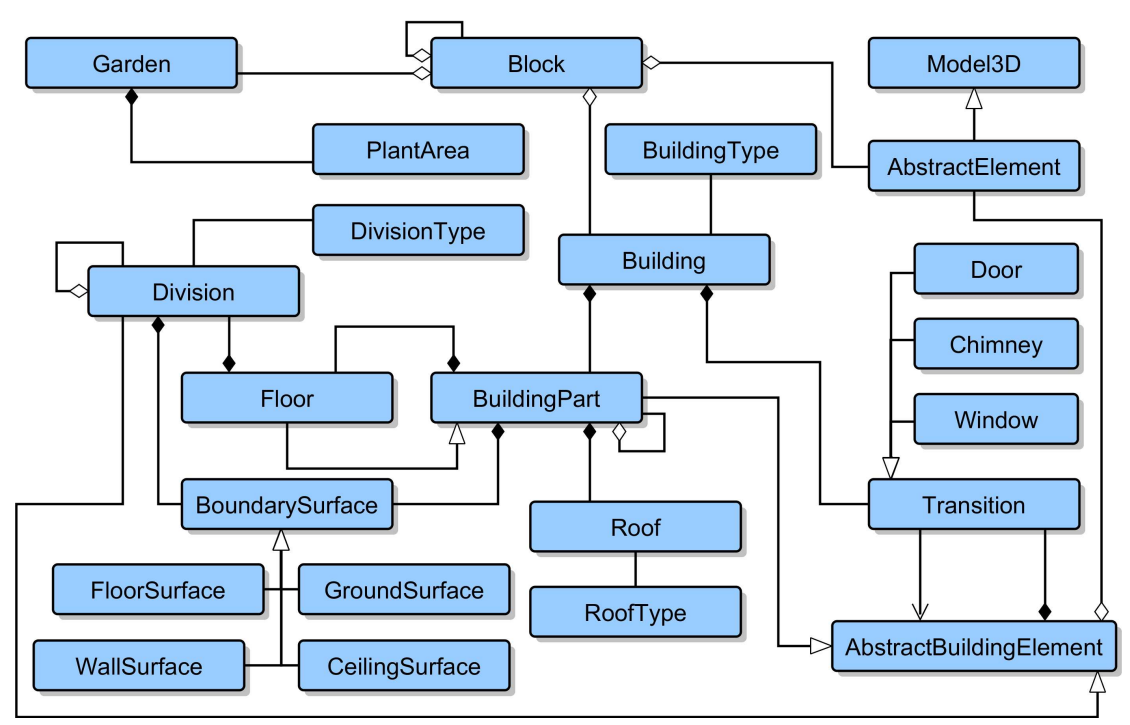

Figure 3.1 - Generic buildings' ontology: an abstract semantic schema that defines the connections between the elements that can be part of a building. A Building is composed by a set of BuildingPart structures; each BuildingPart is a stack of Floor structures; each Floor aggregates a set of Division structures; each Transition relates a Floor and a Division to define a transition to the exterior or two Division elements to establish a inner transition; A Roof is the cover of each BuildingPart; BoundarySurface defines a boundary for Floor or Division structures, as for example walls, grounds or ceilings. Other architectonic structures are considered such as gardens - represented by Garden class - or even 3D models foreseeing the use of complex furniture.

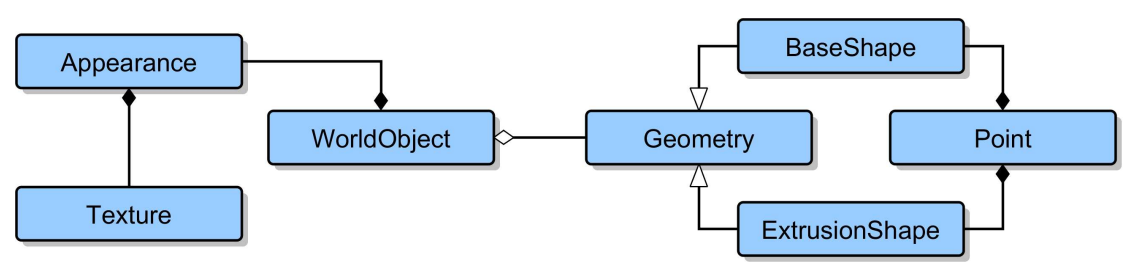

Figure 3.2 - The WorldObject parent and the inheritance mechanism. Each ontology element extends from WorldObject, that contains features common to each element, specifically, the Geometry and the Appearance.

- Building: this is a master container that describes edifications along with their internal elements, such as Floor, Division or Roof type structures;

- AbstractBuildingElement: this is the abstract base element that is contained by the Building class in the form of BuildingPart and Division; 
- AbstractElement: this element represents an abstraction for complementary structures, such as ornaments or furniture that can be present inside or outside the Building (for example tables, chairs, beds, vases);

- BuildingPart: each horizontal composition that constitute a Building is identified as BuildingPart. It can also be specialized into Floor, which form a stack of BuildingPart structures that arise vertically;

- Floor: this class - used to represent a storey - inherits from BuildingPart to compose it vertically. It also holds a set of rooms (represented by Division);

- Division: this structure is used to represent compartments for arrangements or final rooms inside a Floor;

- BoundarySurface: represents each contention structure for divisions and floors. For example, it can be an exterior or interior wall and also a ceiling or a ground;

- Transition: represents a building hole that intends to provide an interface with the building exterior or a passage between a couple of divisions. A Transition can be a door or a window, for example;

- Roof: BuildingPart structures are covered by roofs, that are typically placed upon the highest floor;

- Garden: optionally, a Division may hold a Garden. These structures can also be present in the Building exterior, namely in its containing Block.

The abstractness of the generic ontology foresees the possibility of extending it to other particular cases (e.g. roman buildings), as it will be presented in the following subsection.

\subsubsection{Extending the generic ontology to the roman archi- tecture}

The requirements of the roman architecture are more specific than those used to plan and develop the generic ontology. The existence of structures such as arches 
and columns calls for the specialization of some generic classes, mainly abstract elements. In this work, only a few roman elements were extended to demonstrate the flexibility and scalability of the buildings' generic ontology, through the inheritance mechanism. It is important to state that the roman architecture was not explored in depth to maintain the focus of this thesis. Thus, the elements in Figure 3.3 should only be considered for demonstration purposes.

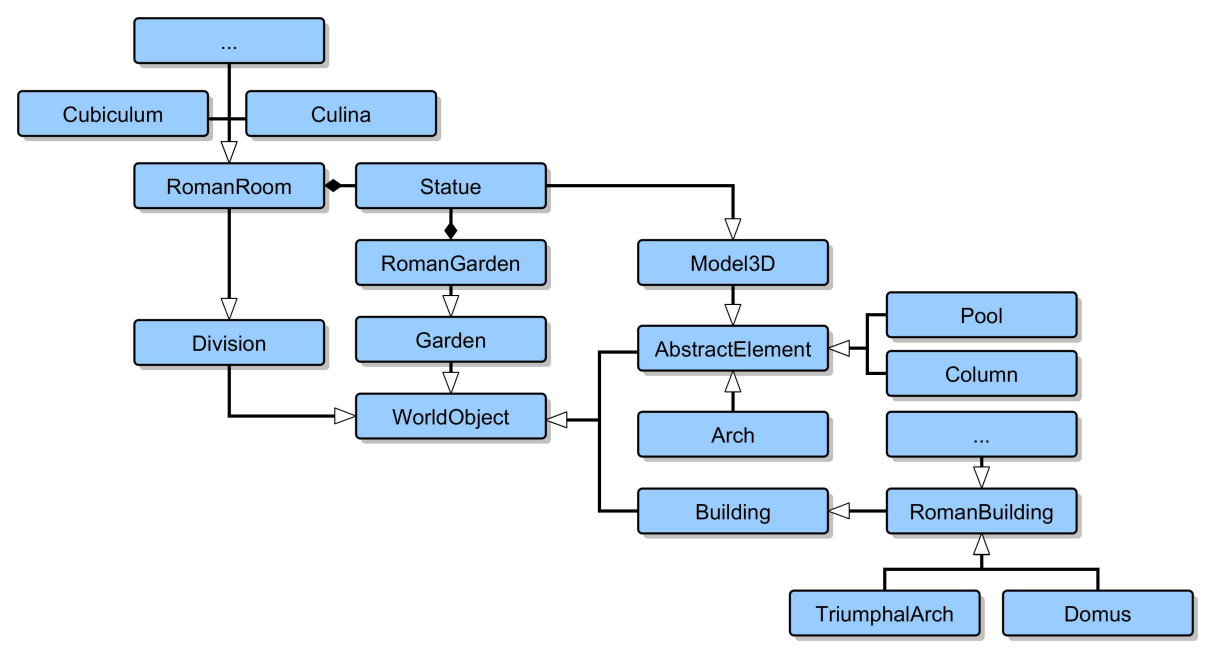

Figure 3.3 - The specialization of the generic ontology into roman architectural objects, by extending generic elements to fulfil some of the roman architecture requirements.

\subsubsection{Roman ontology elements}

The specialized roman classes are described below.

- RomanBuilding: specifies an abstract building belonging to the roman style. A domus is an example of an ancient roman building, used for housing purposes.

- Column: this is very common in the roman style. Column structures were used for decorative or structural purposes, inside or outside the buildings;

- Pool: were used in domus for water recoil and supply. These pools, also called impluviums collected the water through the openings in the roofs and ceilings called compluviums; 
- Statue: this structure represents tributes to ancient roman emperors. The complexity of this kind of structures makes them more suitable to be used as 3D models manually produced, in the context of virtual representations;

- Arch: similarly to columns, this element was very used as ornament in the roman architecture. This work does not cover the reconstruction of it. However, it should be considered as a likely extension example from AbstractElement;

- RomanRoom: this structure is an abstraction that intends to represent a division belonging to a roman building. Culina and Cubicullum, for example, are two division types that can be found inside a domus.

Summing up, the proposed buildings' generic ontology intends to establish a generalist organization for edified structures, based on the CityGML standard. Moreover, it provides the first regulatory set for the procedural modelling methodology that will be presented in the next section. Additionally, it is possible to extend the generic ontology to define the organization of architectural elements for a specific architectonic style (for example, the roman style). Flexibility, scalability and re-usability are some of the advantages that accrue from the adoption of these semantic structures, if one considers the following aspects:

- In the same ontology (and architectonic context), extensions can be made to specialize structures (for example, the proposed generic ontologies stablish that a BoundarySurface extends to WallSurface, FloorSurface, GroundSurface and CeilingSurface);

- Extension/derivation mechanisms also allow adaptation to particular architectonic styles, from previously created ones;

- Associated implementations (regarding, for example, geometry production) can be reused and enhanced. 


\subsection{Procedural Modelling Methodology Overview}

The proposed procedural modelling methodology defines the process to produce virtual representations of one-floored traversable buildings composed by arbitrary shapes. The aforementioned ontology that describes the building entity and its interior components to properly regulate the proposed methodology, can be mapped by auxiliary structures such as a data model (e.g., XML) or a grammar. The resulting rules are then interpreted and transformed into $3 \mathrm{D}$ geometries using a procedural modelling process.

In short, the process begins with having the floor plan assembled, considering inner and outer transitions (doors and windows); afterwards, the walls are extruded, the roof is also generated and placed upon each horizontal building composition (BuildingPart); finally, some details are added to enhance the appearance of the virtual building model.

\subsubsection{Building Floor plan definition}

The initial focus of this procedural modelling process is the production of the floor plan generation that is defined through a treemap structure - encoded in rules that, in turn, establishes the building parts and divisions belonging to a building. Following treemap's structure, the process starts by dividing the building constraint polygon into sub-areas that represent the space reserved for building parts (horizontal compositions). Then, for each building part, a floor is created. Each one of those floors is then subdivided into divisions (or rooms), taking into consideration a set of high-level requirements, that are defined on the existent input structure, i.e., the encoded treemap rules that establish the positions and dimensions used to adapt interior divisions constraint shapes. The next step in the process is reserved for the marking of transitions: exterior ports and windows that constitute the points of connection with the outside of the building and inner doors that ensure connectivity between divisions. Thus, considering the architectural project requirements, access doors and outer windows are properly marked in the marginal divisions that 
are in direct contact with the building's limits. Then, inner doors are also marked upon overlapping walls of communicating divisions. In the end of this stage, some alterations can be made to the resulting divisions, in order to adapt their geometry through the modification of their contour walls. In short, this modification consists in removing or adding points to a given boundary surface that represents the referred division contour wall, as will be explained later with more specificity.

\subsubsection{Extrusion, roofing and completions}

The walls' extrusion and the placement of ceilings, grounds and roofs provide the 3D virtual model, in its final form. The walls' extrusion regards the positions occupied by the doors and the windows. Then, accordingly to the geometric limits of each division, both the ground and the ceiling are placed. The roofs are also generated and placed to cover the building top, considering its building parts. Finally, some features are produced - such as frames for doors and windows, building base and roof skeleton coarsening - to enhance appearance of the 3D virtual building model.

\subsection{Summary}

This chapter provided an overview on the proposed procedural modelling methodology, that regulates the generation process through the defined buildings' generic ontology - partially based on CityGML standard- which is extensible to particular cases such as the roman style. The abstractness of the developed ontology allows its adaptation to specific data formats - such as data models or grammars - that provide input for the modelling process. Such input can be seen as a set of requirements, in which implicit hierarchical structure of treemaps establish arrangements for building parts and divisions (or rooms), respective weights of occupation and connectivity between divisions, guiding the proposed methodology in the production of the desired virtual building model. This is done through the application of the following sequence of steps: floor plan definition, wall extrusion, roof generation 
and placement and, finally, building enhancements through the improvement of element details. The proposed procedural modelling methodology is exposed in detail in the next chapters, along with its different stages and adapted techniques. 



\section{Generation of Virtual Buildings Formed by Rectangles}

This chapter presents the first mature stage of the proposed procedural modelling methodology for generating domus - ancient roman houses - considering rectangular constraint shapes, through the combination of an ontological schema - extended to support some elements of the roman architecture - and a procedural modelling process, that is responsible for creating the geometry according to the rules that define the buildings (Adão et al., 2013b).

This version of the methodology must first receive the proper guidelines containing the building definition rules. Besides their relation with the ontology, a part of these guidelines follow a proper L-System format to enable the creation of a hierarchical treemap, establishing the division and subdivision of the building into building parts and divisions (also known as rooms). More specifically, the L-System approach is used to iterate through the rules to mount the treemap that hierarchically organizes the subdivision of the building into a node system, mapping building parts and their divisions. Afterwards, the splitting of the building floor plan into areas occurs, considering the previous treemap definition: the rectangle that constraints the building is assumed as a root node, that is divided into building parts, where each building part is subdivided into divisions. Division sizes are calculated considering the occupation weights explicitly provided by definition rules, that will 
be addressed further in this chapter. The floor plan generation process is over when transitions are created to properly connect divisions and to define buildings' access doors and windows. Restriction rules are used for this purpose, since they specify connections between division types. Next, the walls are extruded and finally roofs are created. Some typical roman structures were included, such as columns and pools (impluviums/compluviums), to render the virtual houses properly adapted to the roman architecture context.

This chapter is organized as following: firstly, an overview of the proposed methodology (preliminary approach), capable of dealing uniquely with squared floor plans, will be presented. Next, the ontology adaptations and rules that aim to regulate the procedural modelling methodology, at this development stage, through the establishment of requirements and guidelines for building generation will be addressed. The implementation details and preliminary results are exposed in the last sections.

\subsection{Preliminary Procedural Modelling Methodol- ogy Proposal}

In this section, the earlier stage of the procedural modelling methodology for domus generation is presented, considering the roman ontology addressed in chapter 3, definition rules and restriction rules.

The roman ontology results from the extension of some elements belonging to the proposed generic ontology presented in chapter 3. Based on it, an L-System rule format to define treemaps for building subdivision was developed. The referred rule format belongs to the definition rules which also define division occupation weights, division types, building and building part dimensions and roofs. The subdivision process occurs hierarchically: first, building parts are divided and then, for each divided building part, inner divisions are also subdivided. There is another type of rules set - restriction rules - that imposes a complementary level of guidelines: it establishes the types of division that can connect. 
Regarding the procedural modelling methodology, it starts with the floor plan production. Firstly, the subdivision of the building into building parts and division areas, oriented by the aforementioned definition and restriction rules, takes place. Then, considering the second set of rules, adjacent divisions are properly interconnected, ending the floor plan generation step. A sequence of extrusions results in the $3 \mathrm{D}$ virtual model of a domus.

\subsubsection{Ontology integration}

The generation of roman structures requires adaptations to the previously proposed generic ontology. However, its core - semantics and organization - remains unchanged. The main container element continues to be the Building. Typically, buildings are composed by, at least, one BuildingPart, each one holding a single Floor and a unique Roof. In turn, each Floor groups a set of divisions, connected to each other through transitions, that also represent building interfaces, such as exterior access doors and windows.

The required ontology adaptations to support roman structures involve the extension of some of its elements. Thus, to support the particular case of the roman domus, the following main extensions were made: Domus inherits features from RomanBuilding which is, in turn, a Building extension; RomanRoom inherits features from Division, that is a base class for several other specific structures such as Cubiculum, Culina, Vestibulum, Atrium, etc.; to support the generation of divisions similar to atriums with the typical structures for water recoil purposes, the AbstractElement gave origin to Pool which, in turn, represents the Impluvium, a water deposit that looks like a tank; Compluvium is a special Transition, usually aligned with an Impluvium structure, that represents a hole in the roof for water capturing; finally, RomanGarden inherits features from Garden, to support the generation of a backyard inside Perystilium divisions. Figure 3.3 (chapter 3) depicts the aforementioned inheritances. 


\subsubsection{Methodology regulation: definition rules}

Some rules have to be defined to specify a mandatory set of parameters regarding the floor plan generation specifically the treemap that subdivides the building into building parts and divisions, dimensions related with the building layout and building parts, divisions' categorization by functional zones, occupation weights regarding the different building elements and presence of structures inside the divisions.

The first referred definition rules set represent the guidelines to divide the building area - one of the main steps regarding floor plan generation - combining two main concepts: treemaps and L-System. The L-System that specifies the set of building parts, arrangement containers and final divisions is, in fact, an encoded treemap structure which is iteratively and recursively mounted.

The first axiom corresponds to the treemap root node regarding the constraint rectangle of the building. The subsequent symbols can be one of two types: replaceable or final. The former corresponds to building parts (i.e. distinguishable vertical building structures that group division sets) or division containers (i.e. section for arrangement purposes). The later symbol type maps final divisions. The process is considered complete when there are no more symbols to replace. Rules defining the treemap are specified by means of grammar with the following format:

SemSymID: ResultSemSym0, ResultSemSym1, .., ResultSemSymN

SemSym represents the axiomatic symbol that gives place to the resulting symbols that come after a colon punctuation mark (:). In general, symbols enable the distinction of ontological elements, depending on functional zone categorization (addressed later in this subsection). Thus, building part containers are represented by symbols without an attributed functional zone category that point to other uncategorised symbols (expressing other building part containers or final building parts). Final building parts are represented by terminal symbols without a functional zone relation, that point to division symbols having a functional zone category. In turn, division containers and final divisions are expressed by symbols with a functional zone category that are replaceable and terminal, respectively. 
Building dimensions are also specified in the definition rules. The size of the footprint and the maximum building height are defined as the following:

BuildingDimension: value_footprint_x, value_footprint_y, value_of_max_height

Another kind of definition rules aims the categorization of divisions sets (specified in the treemap definition rules) as private rooms, central spaces, inside and access corridors and also stores. Such categorization intends to generalize the division types in functional zones, while establishing an implicit relation with the ontology. The general format for division's categorization is the following:

Category: Division0, Divisiono, ..., DivisionN

In the specific case of roman domus, divisions can be categorized as following: Vestibulum is identified as a corridor; divisions such as Cubiculum, Triclinium, Cullina and Bathroom are considered private rooms; typically, a domus has two central spaces identified as Atrium and Peristylium; Tablinium and Andron are inner corridors; finally there are stores used for business known as Tabernae. Table 4.1 depicts this categorization proposal.

Table 4.1 - Roman domus divisions categorized by functional zones.

\begin{tabular}{c|c|c|c|c|c} 
Category & $\begin{array}{c}\text { Access } \\
\text { Corridor }\end{array}$ & $\begin{array}{c}\text { Central } \\
\text { Space }\end{array}$ & $\begin{array}{c}\text { Private } \\
\text { Room }\end{array}$ & $\begin{array}{c}\text { Inside } \\
\text { Corridor }\end{array}$ & Store \\
\hline Atrium & & $\checkmark$ & & & \\
\hline Cubiculum & & & $\checkmark$ & & \\
\hline Triclinium & & & $\checkmark$ & & \\
\hline Tablinium & & & & $\checkmark$ & \multicolumn{3}{c}{ Continues on next page } \\
\hline
\end{tabular}


Table 4.1 - continued from previous page

\begin{tabular}{c|c|c|c|c|c}
\hline Category & $\begin{array}{c}\text { Access } \\
\text { Corridor }\end{array}$ & $\begin{array}{c}\text { Central } \\
\text { Space }\end{array}$ & $\begin{array}{c}\text { Private } \\
\text { Room }\end{array}$ & $\begin{array}{c}\text { Inside } \\
\text { Corridor }\end{array}$ & Store \\
\hline Andron & & & & $\checkmark$ & \\
\hline Peristylium & & $\checkmark$ & & & \\
\hline Vestibulum & $\checkmark$ & & & & \\
\hline Cullina & & & $\checkmark$ & & \\
\hline Bathroom & & & $\checkmark$ & & \\
\hline Oecus & & & $\checkmark$ & & \\
\hline Exedra & & & $\checkmark$ & & \\
\hline Alae & & & $\checkmark$ & & \\
\hline Servae & & & $\checkmark$ & & \\
\hline Tabernae & & & & & $\checkmark$ \\
\hline \hline
\end{tabular}

Thereby, those division types have to be used when defining a certain roman division, rather than the original designation. For example, a certain symbol specified in the treemap rules with the goal of being an atrium should be declared in this category rules as a central space.

Along with the treemap defining building parts, building dimension and division categories, weights of occupation must be specified, using the following format:

Weights: ElementA-0.5, ElementB-0.5

The keyword Weights marks the beginning of this specification set. In this set, identifier names must be concordant with the elements specified in the treemap specification. Ensuring that, an occupation value for each element can be established using a hyphen mark (-) to separate element from value. The occupation value 
refers to the relative area that is occupied by a certain element inside its parent (it corresponds to the launcher axiom specified in the definition rules). For example, two building parts can occupy a relative value of 0.5 from a building footprint and each building part can be fulfilled by four different divisions, each one taking a relative value of 0.25 from the available area.

There are also the extrusion rules. Keyword BpartsHeight starts a rules section where the heights of each building part can be defined, in the following format:

BpartsHeight: BuildingPart0-valueA, BuildingPart1-valueB, $\ldots$, BuildingPartN-valueX

In this scope, building parts are symbols pre-specified on definition rules that have the respective height value, separated by hyphen (-). The height value is a relation between each building part and the total height of the building, previously defined. Lets say that, if hypothetically a building is defined with 4 of height and a building part has a relative height of 0.8 , this building part has an absolute height of 3.2. Thereby, different heights for the different constituent building parts based on relative values can be defined.

Some divisions might be attached with inner structures such as pools or gardens. To define those divisions and the respective structures, the following rules set needs to be properly specified:

StructureA: DivisionA, DivisionB, ..., DivisionX

StructureB: DivisionC, DivisionD, ..., DivisionZ

The aforementioned rules specify the list of divisions that hold a certain structure (ImpluviumCompluvium and Garden are the supported ones). The structure designation comes before a colon punctuation mark (:) which is followed by a list of divisions (symbols previously defined in the treemap rules).

Finally, roofing rules define which kind of roof covers each building part. The keyword Roof marks the beginning of roof definitions that are specified in the following format: 
Roof: BuildingPart0-RoofTypeA, BuildingPart1-RoofTypeB, ..., BuildingPartN-RoofTypeX

After a colon (:) mark, a set of symbols coupled by hyphens (-) defines, for each building part, the covering type of roof.

\subsubsection{Methodology regulation: restriction rules}

This rules define a complementary set of guidelines and default restrictions used for methodology regulation. Essentially, they specify the set of possible connections between different functional zones categorizing divisions (Figure 4.1) and textures for coating each ontological element.

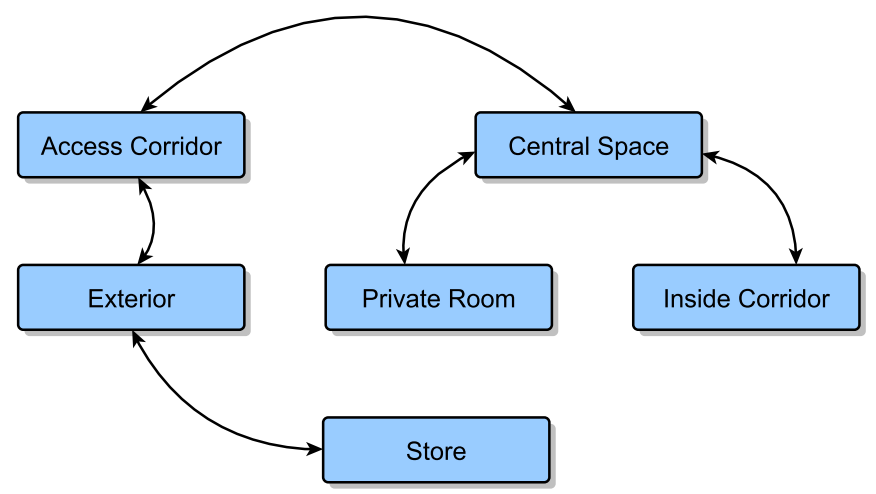

Figure 4.1 - Representative schema of the connections possibilities between functional zones, that regulate the methodology for generating building based on rectangular floor plans.

The restriction set that aims to specify the pair of division types that can connect are encoded as the following template format:

Connections: Category0-Category1, Category0-Category2, ..., CategoryX-CategoryY

Connections are specified after the keyword Connections which is followed by a set of division pairs, separated by comma (,). Each division in a given pair is connected to the other by an hyphen mark (-). 
Finally, texturing rules define the coating of each ontology element. They are specified using the keyword Texture and then, after a colon (-) mark, the pairs of elements and textures as the following template rule:

Texture: OntologyElement0-texture1.jpg,

...

OntologyElementN-textureX.jpg,

\subsubsection{Procedural modelling approach}

The procedural modelling approach uses as input the aforementioned rules and performs a set of steps that aim to achieve a building's virtual representation. The main stages regard the production of the floor plan (subdivision and then, connection between resulting division) and the extrusion of walls. They will be addressed in the following topics.

A) Floor plan subdivision

The first two steps of the floor plan subdivision are related with the treemap - implicitly encoded in an L-System subset provided by definition rules - that aims the subdivision of the building layout into rectangular areas, resulting in building parts and divisions (Figure 4.2). Specifically and considering the subdivision treemap, the first iteration splits the building's initial area into smaller squares which are the building parts. The areas of the building parts are then divided into smaller areas for divisions (which include corridors). The splitting process performs divisions, in each case, accordingly with the relative weights (in percentage), specified in the definition rules.

B) Division connections

When the splitting process is complete, a graph to connect adjacent divisions based on functional zones classification and considering restriction rules is created. This graph is used in the fourth step to mark the openings between connected divisions, that must be adjacent. Afterwards, division wall segments 
interfacing with the building's exterior layout are marked with entry doors for the divisions identified as being access corridors or stores. Moreover, the divisions that have available wall segments placed upon the exterior building layout are properly marked with windows. The next step alters the geometry of each division and each building part to properly adapt to the marked doors and windows: the line that sets the limits of a division is segmented around the transitions. Wall thickness is also applied in this step, along with the marking of roof openings, if needed. These openings are applied to enable the communication between structures such as impluvium and compluvium, that constitute a water recoil system in the context of the roman architecture. The result of these steps is a rule-concordant floor plan.

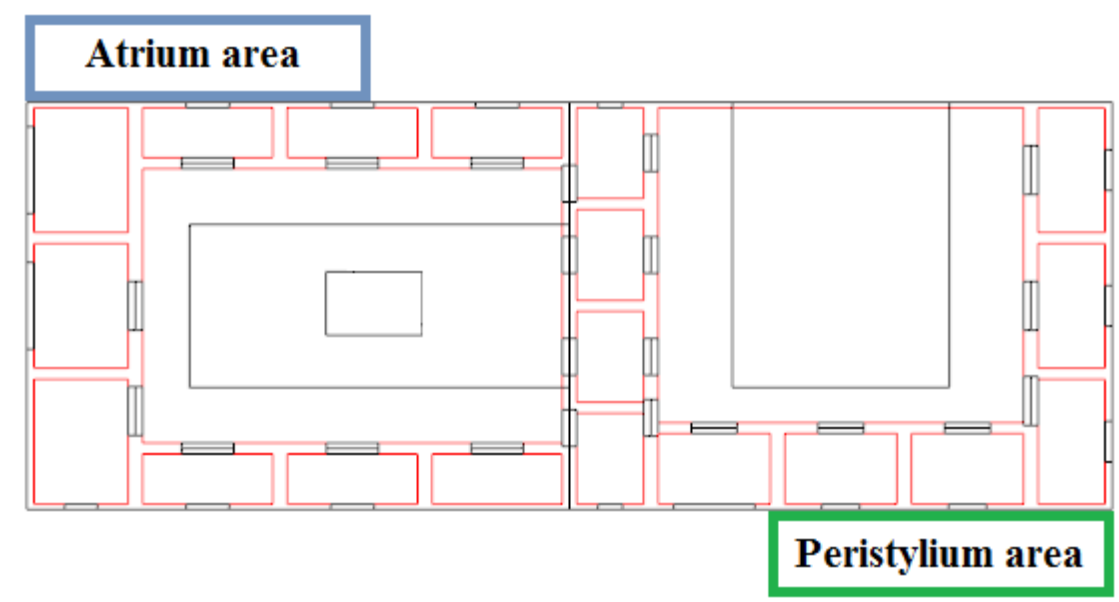

Figure 4.2 - Floor plan generation, using a treemap approach for dividing a roman domus into rectangular divisions: the two first squares generated by the process are the widest areas - typically representing atrium and peristylium zones - and the next iteration splits each areas into smaller rectangles for divisions (rooms).

C) Extrusion process

The procedural methodology finishes its execution by extruding the resulting floor plan, to produce a 3D virtual building model. This process achieves its objective in a few steps. Firstly, divisions' walls and building parts are raised from segments that do not overlap to openings. Then, wall portions belonging to openings are properly extruded: if dealing with a door, a set of walls rises 
to glue the top of the transition to the building's roof base; the same happens for a window, but additional walls are raised to patch the window bottom. The process respects the restrictions imposed by the previously addressed definition rules. Some other elements are extruded to complete the virtual building. For example, columns are raised from the ground to the ceiling of their holding divisions. Finally, the roof is produced through a set of extrusions properly applied accordingly with its type: Hip, Mansard, Flat or Holed.

Figure 4.3 presents a scheme that depicts the aforementioned process.

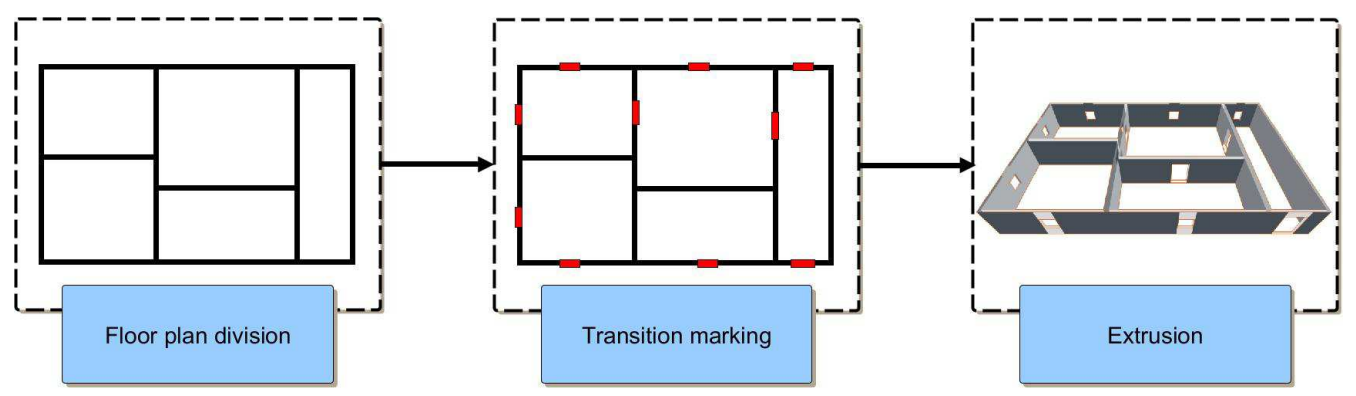

Figure 4.3 - Procedural modelling approach main stages, from the treemap-based floor plan division to the walls' extrusion. From left to right, the first frame depicts the subdivision of a given rectangular footprint, based on treemap. Then, the second frame exposes the flagging of doors and windows. The last frame shows the walls' extrusion stage, that considers the previously flagged transitions.

An algorithmic perspective details the behaviour of each one of the addressed stages. The first one regards the treemap subdivision.

After the proper loading of the tree into a set of ontology-based objects with the data provided by definition rules, the building footprint is determined and BuildingPart structures gain their definitions along with their respective Division structures. Then, this tree is traversed to perform the subdivision. The terms horizontal and vertical (and lexically related) are used to identify a certain orientation in a 2D plane, during this floor plan division explanation. The process starts by horizontally splitting the building footprint into rectangles for BuildingPart structures (direct child nodes of the root node representing the Building). Then, a splitting orientation flag 
is changed to vertical on each one of those BuildingPart structures to alter the splitting direction of their subsequent BuildingPart children, if they exist. The presence of BuildingPart child instances inside a given BuildingPart instance converts the parent into a temporary container for arrangement purposes. Temporary BuildingParts can have many depth levels with more temporary BuildingPart structures that vary the splitting orientation (inverted relatively to its holding structure) in each depth. If a given BuildingPart instance does not possess any BuildingPart child, it is considered a final piece (last depth building part) with a established position and dimension.

The same operating logic applies to the Division structures, defined inside each final BuildingPart instance. They start by splitting, horizontally, each Division instance area. The orientation flag is also changed to enable the divisions arrangement during the subdivision: a feature achieved with disposable temporary Division structures (as it occurs with the BuildingPart structures). The presence of inner child instances of Division converts a given Division parent into a temporary container (subsequent structures are also converted, recursively, if they hold children of the same type). Otherwise, the absence of them indicates that a given division is a final structure.

In short, a building can have one or several temporary BuildingPart containers which, in turn, arrange other temporary containers of the same type or final BuildingPart structures. Each BuildingPart structure may hold several temporary Division containers and the latter can have other structures of the same type or even final Division instances. Division or BuildingPart children represent deeper levels of structures that invert the splitting orientation on those children, from depth to depth.

For both cases, BuildingPart and Division, temporary structures used in splitting operation are removed at the end of the subdivision process because they are no longer needed. Figure 4.4, depicts the generic workflow that addresses the aforementioned operations.

The creation of transitions is the next stage to complete the floor plan definition. Firstly, the division type is checked for each Division inside each BuildingPart. If the 


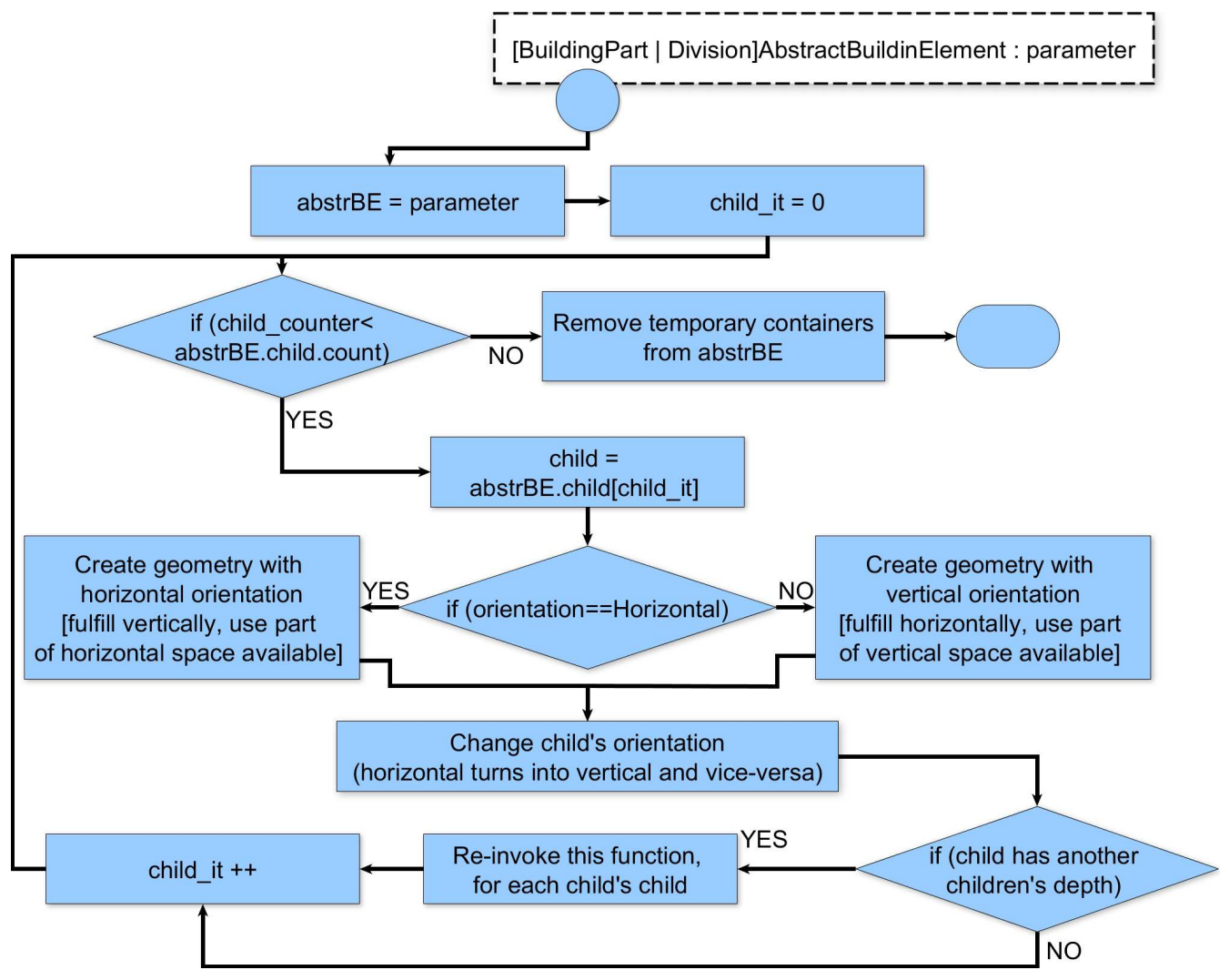

Figure 4.4 - Generic workflow diagram depicting the behaviour of the treemap subdivision process. When a given AbstractBuildingElement structure has child instances of its same type, a rectangular area for each one of those children is produced, considering the respective occupation weights and subdivision orientations (vertical or horizontal). Then, each child is submitted to the same operations to recursively process the children of that child, if they exist. In the end, all parent structures (temporary containers with children of the same type) are disposed while the final structures (without children) are kept. The process works for both BuildingPart and Division structures.

Division is identified as an access corridor or store - division types that require access to the building from the outside - then an entry door is properly created upon the first available division wall segment overlapping with the building constraint polygon. Moreover, the remaining segments that overlap with the building constraint polygon are used to mark windows. Finally, adjacent interior divisions that connect with each other (requirement defined on the restriction rules for connectable division types) are marked with a common interior door. Figure 4.5 presents the behaviour of the transition placement stage. 


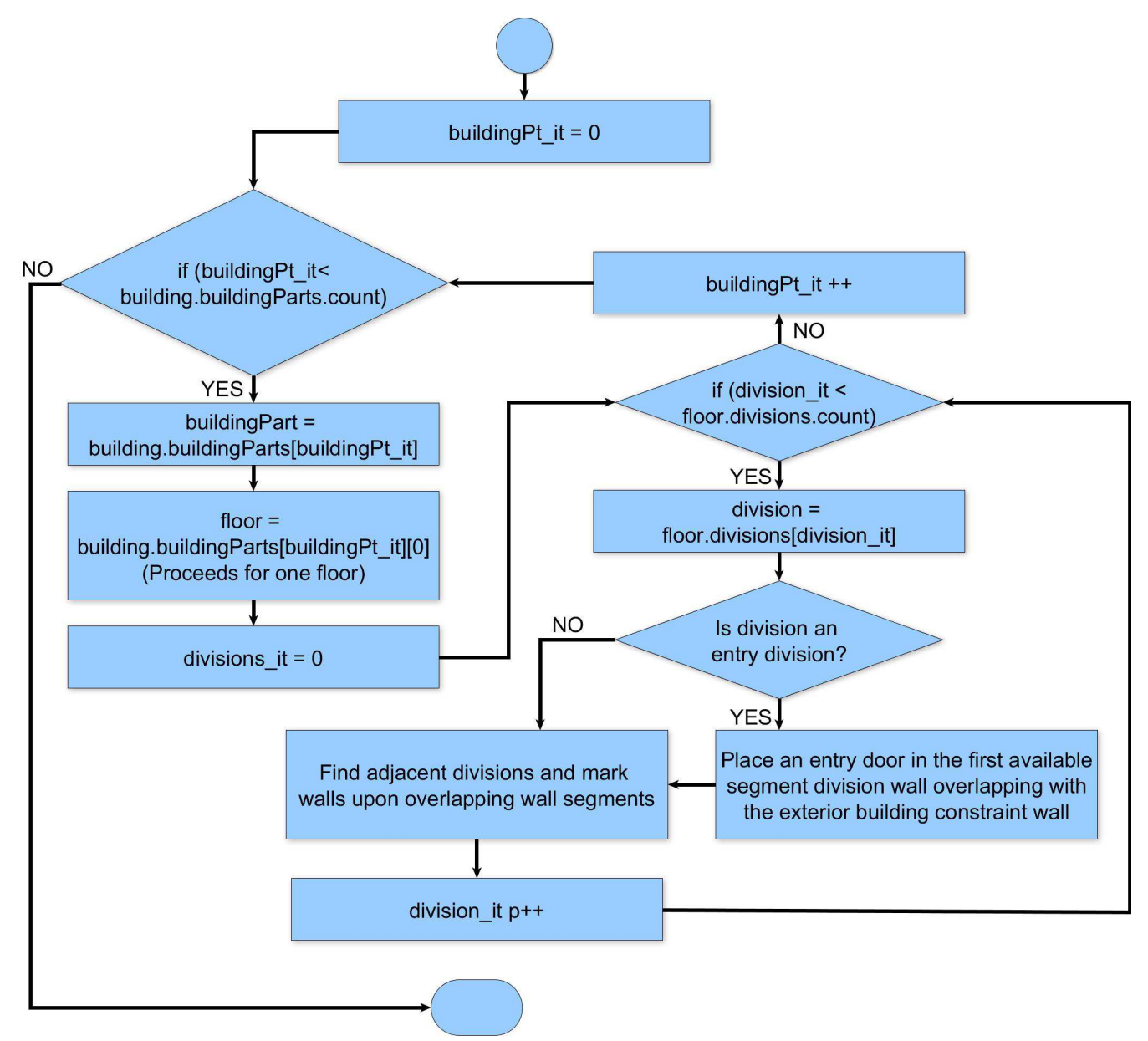

Figure 4.5 - Generic work-flow diagram depicting the behaviour of the transition placement process. The Division structures are traversed inside each BuildingPart to proceed with the placement of entry doors, windows and interior doors. This step relies, mainly, in the identification of overlapping wall segments between two Division structures or between a given Division and the building constraints, to properly create transitions.

During the floor plan generation, other structures might be marked to adapt and enhance the virtual building model. These operations rely in some definition rules regarding the attribution of structures to divisions (addressed previously). A division attributed with an Impluvium Compluvium flag is endowed with both Impluvium (pool for water collection) and Compluvium (roof openning for water recoil) structures which are geometrically marked in the center of the referred division rectangular area, along with a set of surrounding columns. Typically, a domus division with 
such structures is an atrium, in the context of the roman architecture. Moreover, a division required to have a Garden is provided with that homologous structure and the respective geometric definition. Thus, a garden area is geometrically marked in the center of the division rectangle along with a set of surrounding columns. Such domus division is known as peristyliums, in the context of the roman architecture.

After determining the transitions and their positions and dimensions, a set of extrusions takes place. This step enhances the virtual building with a 3D aspect. Essentially, the walls of the constituting BuildingPart and Division structures are raised around the previously marked transitions. Some wall patches are then applied to doors (top) and windows (top and bottom) segments to properly complete the surrounding interior and exterior walls. Figure 4.6 depicts the extrusion process.

Afterwards, some elements are extruded to complete the virtual model. This is the case of the existing Column set and Garden structures. Finally, each BuildingPart instance is covered with a proper roof type, ending the virtual building production.

\subsection{Implementation Prototype}

An application to implement the proposed methodology was developed. In the first interaction, rules must be provided, properly encoded in a simple text file. The text file that contains the encoded rules is composed by two sections: definition rules and restriction rules. The first rules set contains the treemap-based structure for the arrangement of building parts and divisions, occupation weights, building dimensions, building parts heights, roof definitions and attribution of special structures to divisions while the latter restrictive rules set establishes the functional zones that can connect and describe the coating textures for building elements.

The application starts by interpreting the definition rules - with a format based on L-systems - to mount the treemap, that is structured in ontology-based class objects. In other words, the building holds a set of building part containers and final building parts that, in turn, group a subset of division containers or final divisions. Those containers are temporarily used for grouping and arrangement 


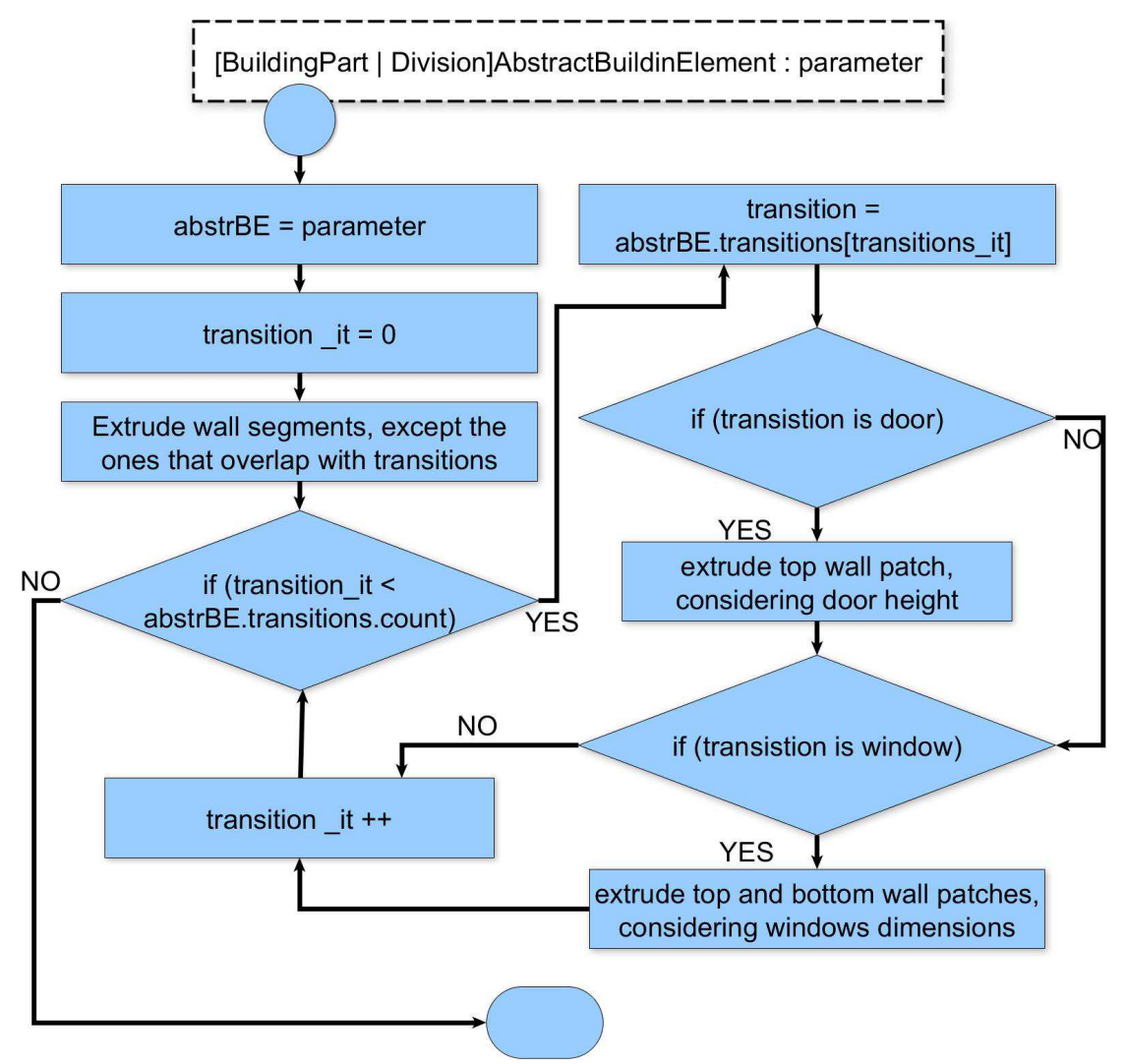

Figure 4.6 - Generic work-flow diagram depicting the behaviour of the extrusion process, for a single AbstractBuildingElement. Each AbstractBuildingElement (Division or BuildingPart) is subjected to an extrusion process that consists in raising its respective walls, considering the previously marked transitions, which have to be patched to achieve the representation of the "wall hole" adapted to both cases windows or doors.

purposes, as it was already underlined in previous sections. Then, the subdivision process takes place: containers, building parts and divisions are split considering the tree mapped into the object classes and also the respective occupation weights. After area provisioning, doors and windows are properly marked in a step that highly relies in the connection rules - a subset of restriction rules - and adjacent division. Thus, for each final division, it is checked - through rules consultation - if its neighbours are suitable for a connection: if so, doors are marked between the proper division pairs that are stored in a "visitation list" for future checkings and redundancy avoidance. Entry doors and windows might also be marked in the division wall segments that overlap with the building constraints (i.e. exterior walls' 
segments), following the process described in previous sections. Next, the walls are extruded respecting the transition marks. Frames for doors and windows are created and finally, roofs are produced. Some additional structures are included - such as columns and pools - to enhance the domus with roman architectural elements.

\subsection{Preliminary Tests and Results}

A domus was defined - using the aforementioned rule sets - to demonstrate the the methodology capabilities in this earlier stage of development. A text file containing definition rules was manually encoded to establish building parts, the divisions belonging to each one, their occupation weights and arrangements, division categorization, and roof definitions. The following excerpt exposes an L-system based specification, defining a treemap for the subdivision of building parts and their respective divisions:

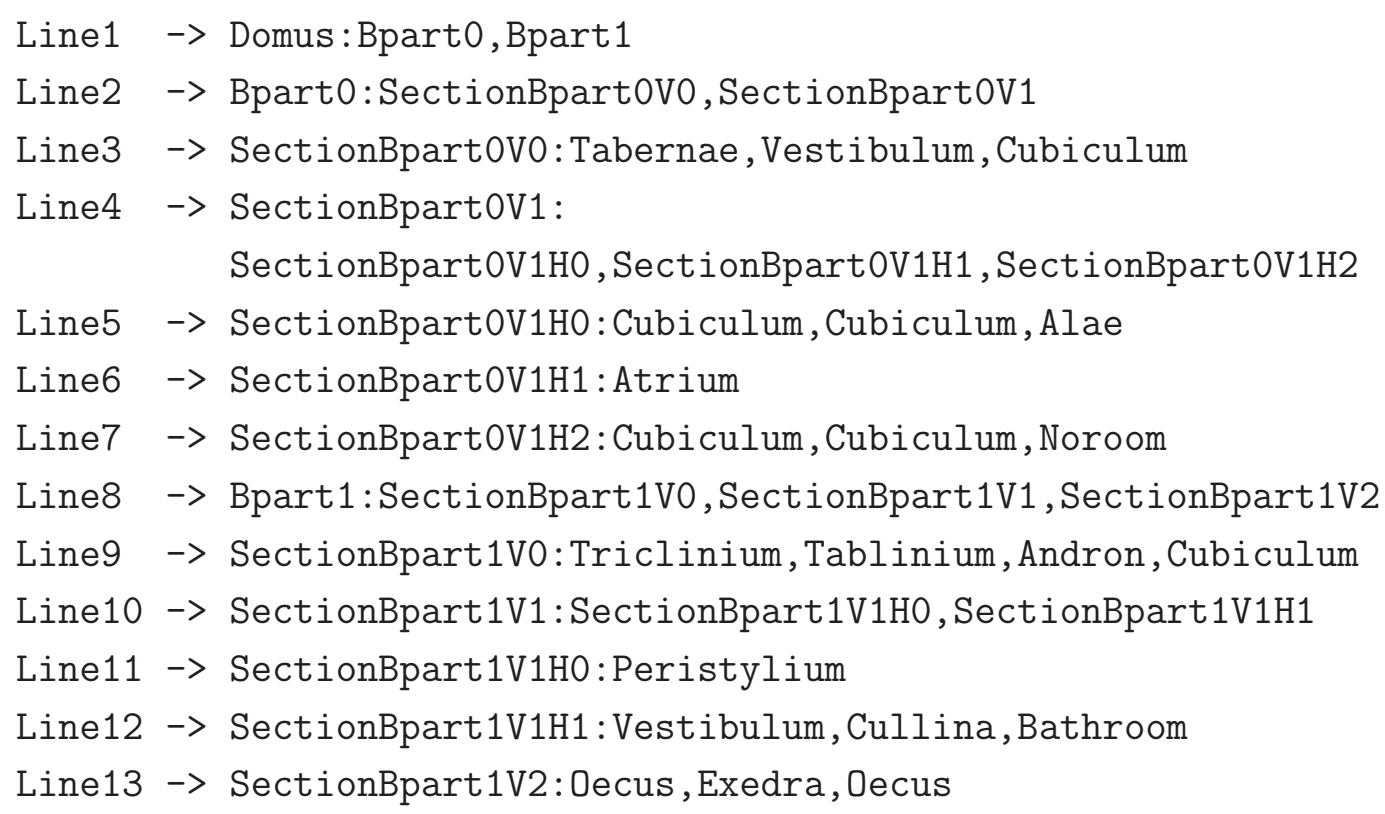

Line1 establishes that a domus will be composed by two building parts (x-axis aligned), Bpart0 and Bpart1. Line2 states the definition for two new auxiliary containers inside Bparto (the alignment resets to x-axis every time a new structure 
type is being divided through definition rules, except for auxiliary containers): SectionBpart0VO and SectionBpart0V1. The former will be populated with three y-axis aligned divisions, as it is defined in Line3. The latter is set to be redivided into new three y-axis aligned auxiliary containers, accordingly with Line4. Line5, Line6 and Line' 7 establish the rules for the referred containers, that are intended to become final x-axis aligned divisions. Line 8 rules define the division of Bpart1 into three auxiliary containers (x-axis aligned): SectionBpart1V0, SectionBpart1V1 and SectionBpart1 V2. The first one will give place to four y-axis aligned roman rooms, accordingly with Line 9 rules. The second is set to have a new redivision in Line10, also aligned with y-axis, which will result in two auxiliary containers. Line11 and Line12 define the set of $\mathrm{x}$-axis aligned divisions that will replace both the last referred containers. Lastly, SectionBpart1 V2 is overwritten with three final divisions or rooms (y-axis aligned), due to Line13 rule.

Other definition rules, such as divisions occupation weights, flags for entry divisions, windows, division connections and roof types, were also specified. After reading the encoded rules, the prototype performs a set of steps - detailed in previous sections - to achieve the 3D virtual model. A rectangular footprint is subdivided into a floor plan using a treemap approach that respects the L-system structure for division arrangement. Then, doors and windows are properly marked on the divisions. Finally, a set of extrusions along with the roof placement, results in the virtual building which is shown on Figure 4.7 .

\subsection{Summary}

In this chapter, an ontology-based solution for procedural generation of ancient structures, specifically roman houses - domus- configured by rectangular floor plans, was presented. The solution implements a working methodology that relies on an abstract ontological specification for buildings, foreseeing the possibility of extension to other architectonic styles. The entire generation process relies in a set of rules definition and restriction rules - to successfully achieve the proper virtual building 3D model. 

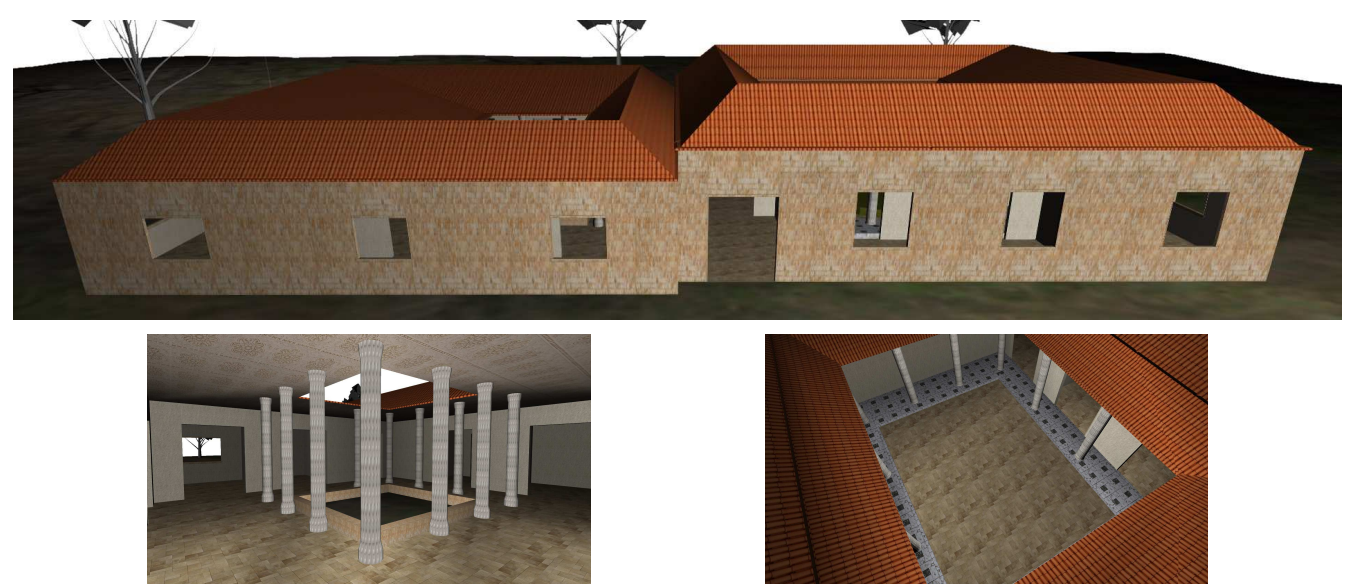

Figure 4.7 - Final layout of a generated domus including interiors and a exterior, using the prototype created to test and validate the proposed preliminary methodology for generating virtual building models based on rectangular floor plans. The virtual model contains a set of domus divisions, including some typical ones representing the atrium and the peristylium.

However, this procedural modelling methodology needs to overcome the key issue related with the treemap approach: the generation of buildings exclusively configured by rectangular shapes. The next chapters will focus on this issues, seeking to contribute with a solutions to overcome them. A XML-based rule system will be also suggested as an alternative for providing virtual buildings' production rules. 



\section{Generation of Virtual Buildings Constrained by Convex Shapes}

A procedural modelling methodology incorporating a treemap approach to generate virtual buildings constrained by rectangular shapes and composed by rectangular divisions was presented in chapter 4 . In this chapter, the aforementioned methodology was enhanced to support the subdivision of buildings constrained by convex shapes with a stronger focus in the geometric operations (Adão et al., 2013a, 2014).

Similarly to the first proposed procedural modelling methodology version, some input definition rules must be provided to trigger the building's generation process. Such rules must fit the requirements of the ontology-based data model, which gathers a set of essential building informations. Moreover, a process to moderate input rules is suggested to make some preliminary validations: checking for rules errors (format and typos) and pre-testing of transitions at a geometric level (a probing approach that uses floor plan subdivision and division's size adjustments to check the viability in placing transitions to connect divisions according to the rules). The resulting ontology-based class set, properly loaded with validated rules, passes through the procedural modelling process that, in turn, builds the expected virtual model in the following sequence of steps: constraint polygon determination; division area provisioning considering division classification, which is an extended feature for division type differentiation (e.g., kitchen or living room); placement of windows and 
exterior access doors; placement of transitions between divisions; roof generation; placement of ceilings and floors and finally, virtual model detailing.

The proposed procedural modelling methodology was implemented for concept-proof purposes, resulting in a software system. The system's input relies in an XML file properly loaded with building's data. The XML format was wittingly taken from the aforementioned ontology-based data model to establish a well-defined structure for buildings specification and is designated by eXtended Markup Language for Building Definition (XML4BD). This format main goal is to overcome some issues related with the first methodology version input rules which were complexly fragmented and partially misaligned with the proposed ontology. So, after the input of a XML4BD file to the system, file's content is read and verified by a module to moderate definition rules. The same moderator module loads the properly validated XML4BD data into an ontology-based class structure and performes the process for probing the floor plan generation: it consists in balancing divisions (or rooms) to try to force them to have a suitable size for doors placement. Afterwards, a procedural modelling module is used to produce the final form of the $3 \mathrm{D}$ virtual building.

Regarding this chapter's organization, it starts by presenting the enhanced procedural modelling methodology, followed by its system implementation. Finally, some preliminary results are presented to assess the system's capabilities in reconstructing roman buildings from real ruins and also hypothetical fictitious structures.

\subsection{Enhanced Procedural Modelling Methodology Proposal}

The previously proposed procedural modelling methodology (chapter 4) was only capable of generating virtual buildings based on rectangular floor plans and also composed by rectangular divisions. In this section, some improvements to that methodology are going to be presented, namely those concerning the definition rules and the treemap-based subdivision process of the building's constraint polygon. These improvements enable the generation of virtual buildings constrained by arbitrary 
convex shapes.

\subsubsection{Ontology-based data model to guide definition rules}

The definition rules proposed on the past chapter 4 are effective in specifying virtual buildings. However, the used format is complex because involves a lot of required definitions with a considerable amount of parameters specified using some heterogeneous formats: firstly, an L-system based specification has to be defined with the treemap of temporary containers (symbols that express arrangement purpose structures), building parts (terminal symbols without a functional zone category) and divisions(terminal symbols with a functional zone category); those specified divisions must be categorized by functional zone as well as division containers; each one of those elements has to be properly configured with occupation weights; buildings and their constituting parts require the specification of dimensions (heights, widths and lengths); roofs were also required for definition. The whole information set has to be defined using several subsets of non-intuitive grammar-based rules which have a structure that is not clearly engaged with the proposed ontology. Besides, there are no mechanisms for validating them. Thus, in an attempt of simplifying virtual buildings definitions and make them prone to proper validation, an ontology-based data model was established with a straight structure and a clear field set regarding buildings data. Two main achievements are reached with this strategical change: the specification based on L-system structures was replaced by the ontology-based specification which relies in the establishment of explicit node hierarchies between the several elements composing a virtual building (temporary containers, building parts, and divisions); finally, occupation weights are automatically populated, based on a size factor defined only for final divisions (explained in detail in later sections) in order to replace the tedious task of defining occupation weights for each building element, individually.

The aforementioned simplified ontology-based data model was designed considering a set of fields that was identified as having the essential information about a building. As it might be noticed in Figure 5.1, its entity classes and organization respect 
the ontology defined for buildings, from which it derives. A Building represents the major container of the scope, since every other objects are, directly or indirectly, supported by it. A Building instance holds at least one or more BuildingPart instances. In turn, each BuildingPart instance may hold one or more Floor instances (however, this work assumes that buildings with only one floor will be addressed). A Division instance is, essentially the most elementary compartment of a building: an interior room with a purpose (e.g. sleep or cook). In turn, one or more Division instances are grouped by Floor instances. Moreover, a division can aggregate structures: Pool and Garden are the ones currently supported.

For arrangement purposes both Division and BuildingPart can hold spawned instances of the same entity class: a Division container can be temporarily used for arranging other Division instances (temporary or final) inside it as well as BuildingPart containers are able to do with other inner building parts. Finally, Transition represents windows and doors. They compose BuildingPart and Division instances to let them keep track of their transitions. Each Transition also keeps track of the structure pairs sharing it (more relevant for interior divisions).

The presented data model intends to constitute a guiding structure for the specification of definition rules. Hereafter, the fields contained in each entity class will be exposed and briefly explained.

\section{A) Building}

Building is the main container structure and it is composed by the following fields:

- ID: represents the unambiguous identifier field;

- name: refers to the building name;

- description: refers to the building description. It can be used as a complementary information field;

- type: identifies the building type (e.g. habitation house or commercial building). 


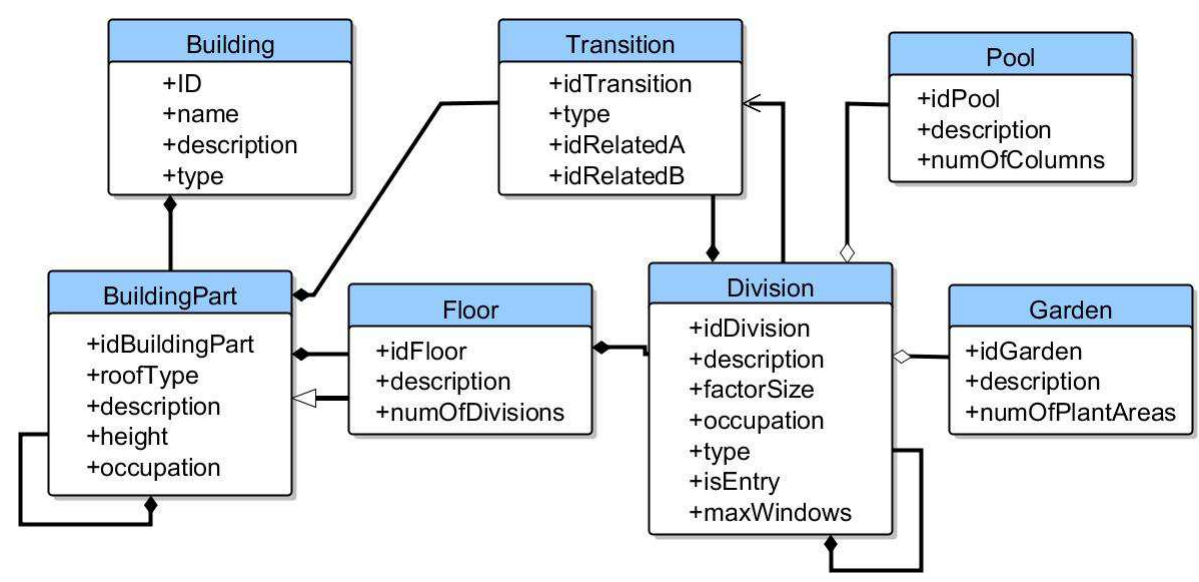

Figure 5.1 - Simplified ontology-based data model. In concordance with the building ontology, it is established that a Building holds a BuildingPart. In theory, a BuildingPart supports one or more Floor instances. However, the proposed procedural modelling methodology only covers the generation of buildings composed by a unique floor. Floor, in turn, is composed by a set of Division instances. Transition instances are shared and tracked by BuildingPart and Division instances. Pool and Garden structures can make part of a division. There is also the possibility of creating BuildingPart and Division containers to arrange other BuildingPart and Division instances inside those containers, respectively.

B) BuildingPart

A building part represents the horizontally displaced parts of the building (distributed at the ground level). It can also be seen as a sub-container, holding floors. This entity class is composed by the following fields:

- idBuildingPart: represents the unambiguous identifier field;

- roofType: defines the type of the covering roof. The supported types are Flat, Pyramide, Hip, Mansard, MansardHoled and None;

- description: refers to the building part description. It can be used as a complementary information field;

- height: specifies building part height (useful for extrusions);

- occupation: refers to the weight of occupation, relatively to the available floor plan area (value determined with the set of divisions).

C) Floor 
A floor is a container for divisions (or rooms). The fields specified in this entity are:

- idFloor: represents the unambiguous identifier field;

- description: refers to the floor description. It can be used as a complementary information field;

- numOfDivisions: it is a numeric field that can be set with the number of supported divisions.

D) Division

Division is one of the most elementary structures inside a building, consisting in the interior portion of the floor. Usually, they connect to other structures of the same kind foreseeing the mobility inside the virtual building. Its structure includes the following fields:

- idDivision: represents the unambiguous identifier field;

- description: refers to the division description. It can be used as a complementary information field;

- factorSize: refers to the factor used to grow a division area inside the available floor plan area;

- occupation: refers to the weight of occupation, relatively to the available floor plan area (obtained from growth operations using factorSize);

- type: the divisions might be generic rooms, bedrooms, living rooms, corridors, kitchens, toilets or even temporary containers;

- isEntry: this is a flag that indicates if a division instance is an entry division or not (accessible from the exterior).

- maxWindows: refers to the maximum windows number that a given division can have. However, the effective number of windows can be lesser, depending on the number and length of the available wall segments overlapping the building's constraint shape (explained with a bit more detail in later sections). 
E) Transition

Transition instances define the interior doors connecting two adjacent divisions, entry doors or windows:

- idTransition: represents the unambiguous identifier field;

- type: window, door or entry door are the supported types that particularize a transition;

- idRelatedA: specifies the first entity instance (e.g. Division) involved in the transition sharing;

- idRelatedB: specifies the second entity instance involved in the transition sharing;

\subsubsection{Definition rules}

To define a building, a set of definition rules must be provided in conformity with the proposed ontology-based data model. These rules aim to specify a set of nodes with a hierarchical organization. Each node can represent a building part a division or even a temporary container for arrangement purposes (for divisions or for building parts). All of them have a set of required fields that constitute parameters used in the procedural generation stage. For example, a node representing a final division must define an id, size factor, occupation value (it can be set with 0 since it will be supplanted with a value resulting from the operations regarding the growth and balancing of rooms based on size factor, explained with higher detail in later sections) division type (room, bathroom, kitchen, etc.) and also an entry door flag (true or false whether an access door to the building exterior is required or not, in a given division). A further section reserved for methodology implementation will present a concrete XML format for buildings definition, structured in concordance with the addressed data model. 


\subsubsection{Restriction rules}

Restriction rules were defined, in chapter 4, as belonging to a complementary grammar that is used to specify textures for building elements and allowed connections between division types. These rules, which became independent from definition, are now represented by default parameters used by the procedural modelling methodology in strategic steps of building's generation. The list bellow specifies them, briefly:

- Texture parameters (based on the generic ontology): are used to define the coating texture of each ontology element.

- door height occupation: percentage of door vertical occupation in the wall;

- door wall occupation: percentage of door occupation in a wall segment considering as a reference the building layout area;

- window small height occupation: percentage of vertical occupation of the wall for small windows;

- window small wall occupation: percentage of occupation of a wall segment for small windows, considering as a reference the building layout area;

- window height occupation: percentage of vertical occupation of the wall for default windows;

- window wall occupation: percentage of occupation of a wall segment for default windows, considering as a reference the building layout area;

- base exterior occupation: percentage length of the building foundation base considering the interior wall as a reference;

- footer interior occupation: percentage length of the footer considering the interior wall as a reference;

- wall exterior occupation: percentage length of additional walls considering the highest building part as a reference; 
- column height percentage elevation: percentage length of columns considering the container building part as a reference;

- scale thickness: value used for the scale of the interior outlines relative to the exterior layout (which results in the gap between these walls that represents the wall thickness);

- small room default: relative size factor for small rooms, considering the building layout area;

- small room min: min. of occupation factor that a small room may achieve;

- small room max: max. of occupation factor that a small room may achieve;

- medium room default: relative size factor for medium rooms, considering the building layout area;

- medium room min: min. of occupation factor that a medium room may achieve;

- medium room max: max. of occupation factor that a medium room may achieve;

- big room default: relative size factor for big rooms, considering the building layout area;

- big room min: min. of occupation factor that a big room may achieve;

- big room max: max. of occupation factor that a big room may achieve.

As it can be noticed, connections are no longer established by restriction rules (or default parameters). Instead, they are specified in definition rules for the sake of building definition freedom. 


\subsubsection{Moderation process for definition rules}

Definition rules moderation constitutes the first regulation instrument before a virtual building model generation. It is responsible for reading input rules and for validating their structure against the ontology-based data model. Afterwards, a class set that maps the ontology accordingly with the data extracted from the input rules is loaded. This step has a pre-test that relies on floor plan probing to check its feasibility. More specifically, this step intends to ensure that every connectable divisions supports a transitions door. Otherwise, transitions are dropped for connectable divisions that do not share an adjacent wall with sufficient length to have it.

Floor plan probing is achieved in three main steps: firstly, all virtual building elements (including divisions, building parts and temporary structures) occupy a floor plan area that is based on their size factors; then, the first floor plan subdivision takes place; lastly, a step responsible for balancing divisions' occupations takes place, to validate the placement of transitions between connectable divisions. These steps are presented in detail hereinafter:

A) Setting the occupations of virtual buildings elements based on size factors: firstly, floor plan's occupation areas are properly set among divisions considering their size factors, i.e. occupation areas are increased and adjusted using jumps of size factor until the sum of all occupations equals 1. Moreover, division sizes affect their wrapping structures (building part containers, final building parts and division containers), recursively: the occupation value of each wrapping structure is obtained adding up their inner structures' occupation values (provided by final divisions to all the other wrappers), as shows equation 5.1.

$$
\text { container.occupation }=\sum_{i=0}^{n} \text { container_child }[i] . \text { occupation }
$$

B) Floor plan subdivision: this step must be highlighted as it is extremely relevant to the enhanced procedural modelling methodology. It is used to create 
areas for divisions and building parts accordingly with their previously calculated occupations. Since convex shapes are not controllable as rectangles, some adjustments are required to achieve the approximated occupation area inside the restriction polygon. Such adjustments involve shape intersections, division area measurements and small increments and decrements to the division areas. This will be explained in detail in the next subsection.

C) Balancing division weights (to try) to ensure transitions: entry and connecting divisions are tested to check if their weights and the arrangements are properly set up. This checking operation consists in verifying if all of the connecting divisions own a common shared wall with enough space to place a door. Moreover, divisions weights can be increased and/or decreased dynamically using a "borrow space" approach to try to satisfy the referred condition. Problematic transitions, which failed the checking operation are suppressed to avoid further geometrical issues in the procedural modelling process. A failed check occurs when two connecting divisions reach their expansion limit (minimum and maximum limits are provided by a subset of restriction rules) and still, there is no space for a transition. Figure 5.2 depicts the process in a generic workflow. Lastly, divisions' occupation values in the floor plan are recalibrated with the new definitions for temporary containers and building parts.

\subsubsection{Procedural modelling generation process}

After the moderation process validating input definition rules to ensure the proper loading of the ontology-based class set and after probing and adjusting the building's floor plan definitions, the procedural modelling process takes place and starts by producing the floor plan for a given restriction polygon. Each building element which can be a container for arrangement purposes, a building part or a division - is placed and adjusted to the building constraint convex polygon. Essentially, the process follows a treemap subdivision with some intersection and dimensioning operations to deal with the division of such convex polygon. 


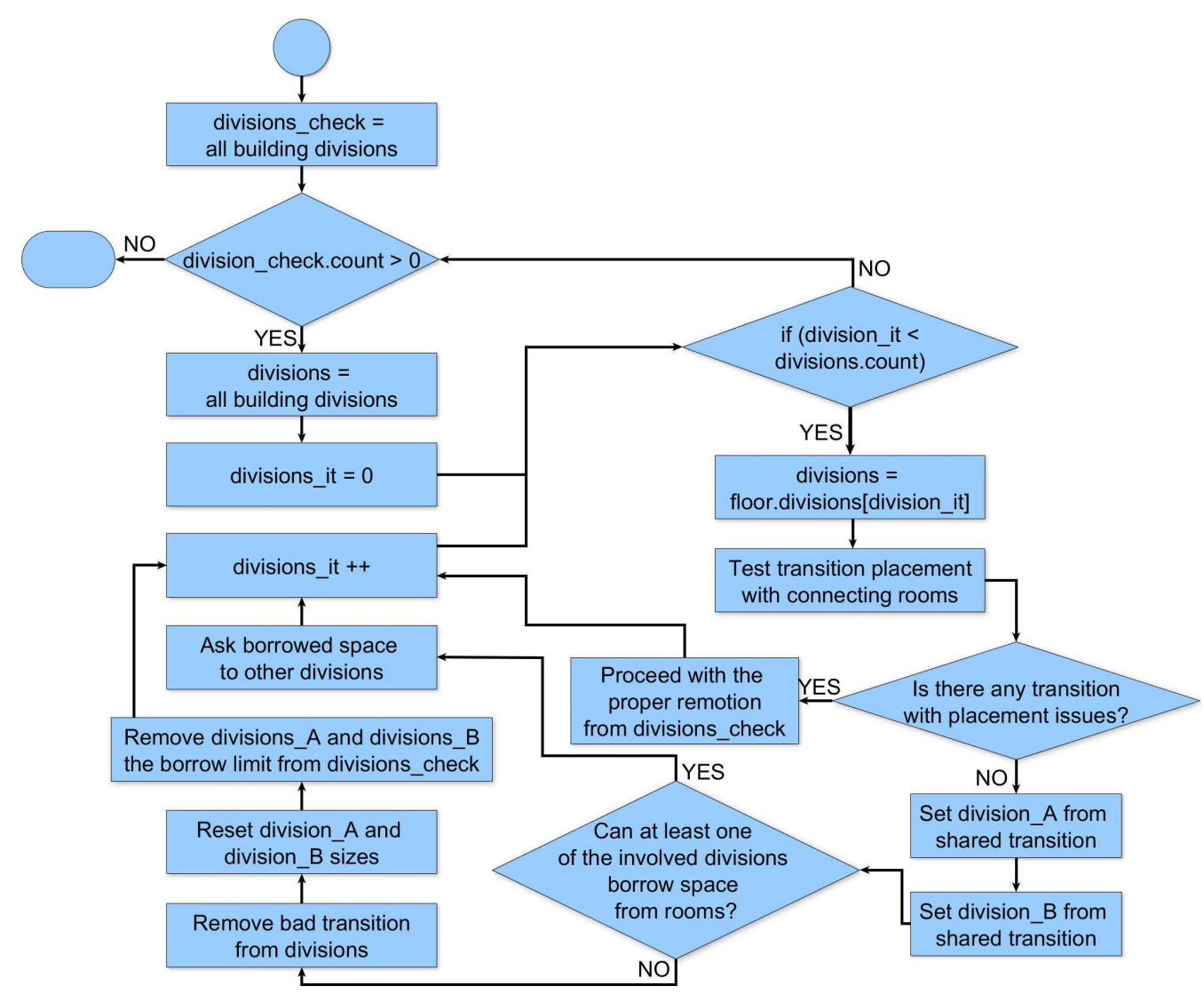

Figure 5.2 - Workflow depicting the process of balancing division weights through space borrowing. Initially, a check list with the complete final virtual building divisions is loaded for control purposes. Then, each division in the building is subjected to the transition placement test which involves a given division (in the iteration) and also their neighbours. If the test does not return any problematic transition (lack of space in placing), the tested division is removed from the check list along with the neighbour divisions with exclusive connection to the tested division. Otherwise and if at least one of the involved divisions support expansion, a small portion of space (based on size factor) is asked to other divisions. To lend space, requested divisions must ensure that they support area decrease (based on the limits established by the restriction rules), without impacting their own transitions. On the other hand, if the tested and connected divisions already reach their limit for expansion, they are both removed from the check list, sizes are rolled back and returned to their origin divisions and the problematic transition is suppressed. The process is repeated until the division check list is empty.

The footprint division process is iterative and begins with a rectangle, for each iteration and building element. The area of this element's initial shape is calculated with the occupation weight value, previously obtained in the rules moderation process. 
However, an intersection has to be made in order to adapt the element's rectangular shape to the virtual building layout, which can result in a partial loss of element's area. This occurs when the rectangular shape overlaps the border of the building's constraint polygon. Afterwards, Heron's formula is used to calculate the area of the new element's shape resulting from the intersection (through internal triangles): if the current occupation weight is lesser than the weight required by the rules, then the process is repeated from the first step, with a wider rectangle (dimensioned with an increment of $0.1 \%$ of the virtual building layout area). Otherwise, the process is considered finished for the current virtual building element and the next one is processed with the same set of operations.

To achieve the complete floor plan, doors and windows are marked considering the division graph provided by the input definition rules, similarly to which was described in the previous chapter. Thus, interior doors are placed on the overlapping wall segments belonging to pairs of connectable divisions, while access entries and windows are placed in the margins of the building constraint polygon, regarding the flagged divisions for this purpose.

The extrusion of the elements provides a 3D model definition. The process is highly based on the previously determined floor plan. Thus, inner and outer walls are raised until a certain height, provided by input definition rules. Then, frames for doors and windows are created along with the roof. Some details such as roof skeleton coarsening, building base and division footers are also created to enhance the virtual model realism.

This summarizes the procedural modelling generation process. Each referred step will now be presented in greater detail:

\section{A) Restriction Polygon}

The polygon that constraints the virtual building is provided by input rules and used to wrap interior divisions. This polygon consists in a list of points that follow a $(x, y, z)$ format. The detection of a non-convex polygon triggers a convex-hull operation to force this requirement. 
B) Floor plan subdivision: combining treemap and greedy approach The floor plan is achieved in two stages: firstly, a convex area is split to produce the divisions' layout and then those divisions are connected. The ontology-based class structure (previously loaded) follows a treemap organization that provides informations on how to split the area by specifying a hierarchical organization for the subdivision operation and the respective occupation weights. Treemapbased floor plan subdivision is properly adapted to deal with the irregularity of the constraint polygon in this proposed enhanced methodology.

In this stage, the virtual building constraint polygon is recursively split to adapt building parts and divisions, following a recursive treemap approach. Next, a more detailed explanation about the splitting process is presented.

Let a given building part $\mathrm{A}$ in the treemap-based class structure be waiting to be partitioned in its child divisions. Let the orientation of the process be vertical and let A be described by a convex geometry coincident with the building constraint polygon. For each child division in A, a rectangular area is created considering its occupation weight (relatively to the virtual building area which, in this example, is the same as A area). This rectangular area is set to fill the available horizontal area and to use a part of the vertical area to partially or totally fit A space, depending on the child division weight value. Then, the rectangle is affected with the intersection result of the current division shape with the containing element ( $\mathrm{A}$, in this example) shape, in an operation that is prone to losing division area, specially in the building layout margins. Such possibility calls for an area compensation process. The proposed one relies in iterative dimensioning operations, until the desired division area is reached. Thereby, the post-interception shape area is measured using Heron's formula (5.2), which relies in the internal triangles' areas calculation:

$$
A \Delta=(\sigma(\sigma-\alpha) *(\sigma-\beta) *(\sigma-\chi))
$$

, where $\alpha, \beta, \chi$ represent the lateral sizes of each triangle and $\sigma$ the semiperimeter given by equation 5.3:

$$
\sigma=(\alpha+\beta+\chi) / 2
$$


If the resulting area occupies less than the specified current division weight, a wider rectangle is created for the division and tested, from the placement and intersection stages. This is a cyclic process that lasts until the division area reaches the proper weight inside the container layout and consequently, within the building constraint polygon (Figure 5.3). Afterwards, the process is also applied for every other divisions in this vertical container, changing their splitting orientation to horizontal, as it was previously explained in chapter 4, during the description of the treemap approach. If they hold some inner divisions to split, the process occurs with other splitting direction. Nodes belonging to these child nodes follow the same splitting process with vertical orientation and so on, recursively and iteratively, until the end of the treemap-based class structure.

The process to produce the floor plan layout is exemplified in the Figure 5.4. It depicts a treemap definition and the respective area splitting process occurring upon the virtual building constraint polygon. Figure 5.4 (a) depicts a treemap schema based on data loaded from definition rules. Figure 5.4 (b) depicts the process of floor plan subdivision guided by the previously presented treemap definition.

C) Placement of transitions

After dividing the virtual building layout, the entry doors and windows are marked in the common wall boundary between the building limits and the division limits. For each division, it is verified if the entrance door is required (flag provided by definition rules). If so, a door is marked upon the shared wall between the current division and the virtual building outline. Then, the windows are also placed in the common shared wall, considering the available space and also avoiding windows overlap. If the shared wall is too small to place the planned number of windows, a fitting subset is achieved through the suppression of the remaining windows. Moreover, inner divisions are also connected by doors, accordingly with the graph provided by definition rules, in the earlier stages.

D) Virtual Model Completions 


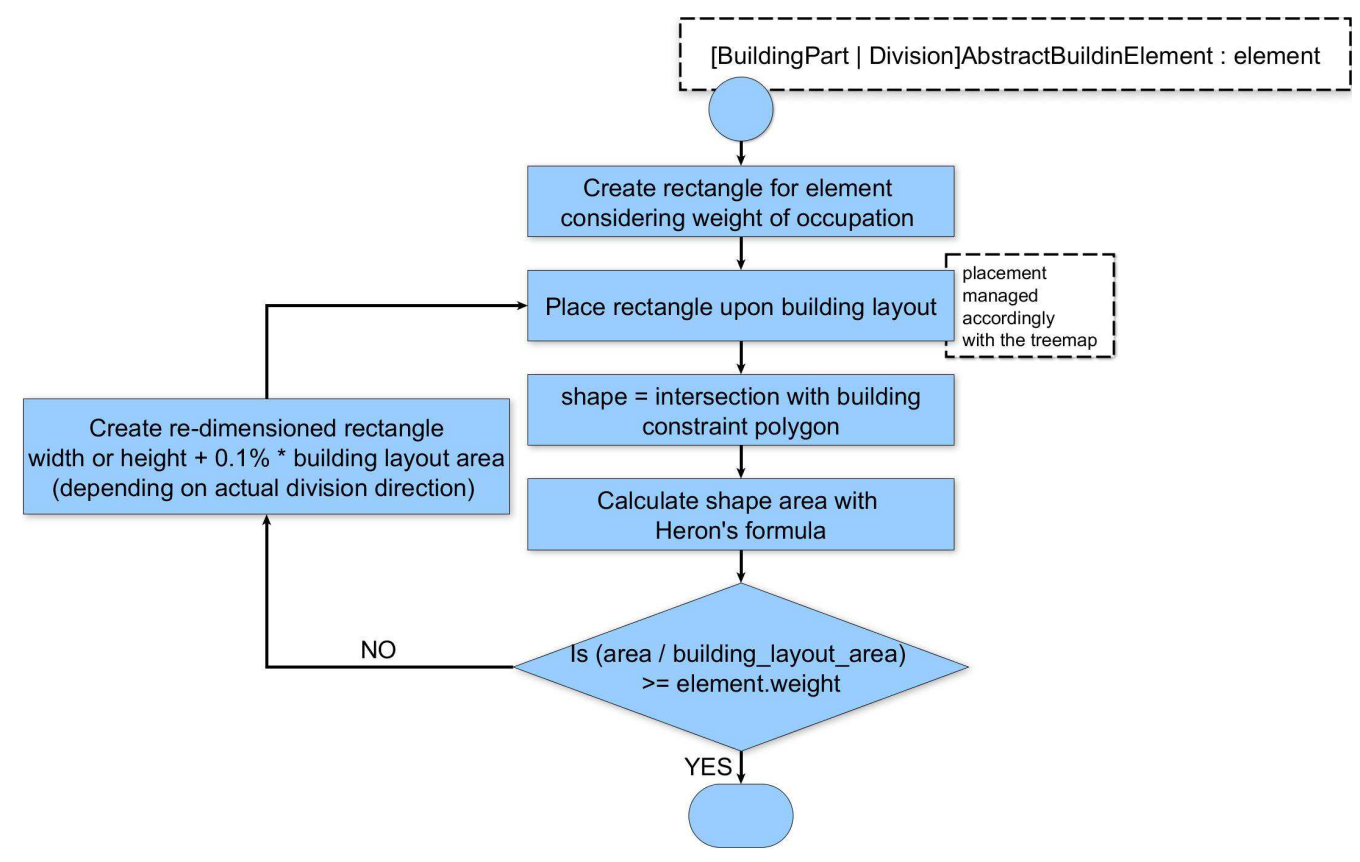

Figure 5.3 - General workflow depicting the adjustments made to a given building element during the floor plan division process of a virtual building constrained by a convex shape (valid for building parts, divisions and temporary containers). In the first place, a rectangular area is created considering the element's occupation weight. Then, the rectangle is intersected with the building's constraint polygon, resulting in a new polygon. The intersected polygon area is calculated to determine if it has the desired size: if so, the process ends; otherwise, the process is reapplied using a wider rectangle (that grows in each iteration), until the desired building element area is reached.

The walls' extrusion and the placement of the ceiling, ground and roof provides the 3D final form. The walls' extrusion operation considers the positions occupied by doors and windows. Thus, the extrusion only happens around the defined transitions. Then, accordingly to the geometric limits of each division, the ground and ceiling are placed. The roof is also generated with the proper dimensions and placed at the top of the virtual building (individually for each building part) to cover it. Finally, some features - such as doors and windows frames or building base - are added to the 3D virtual model to increase its realism. 


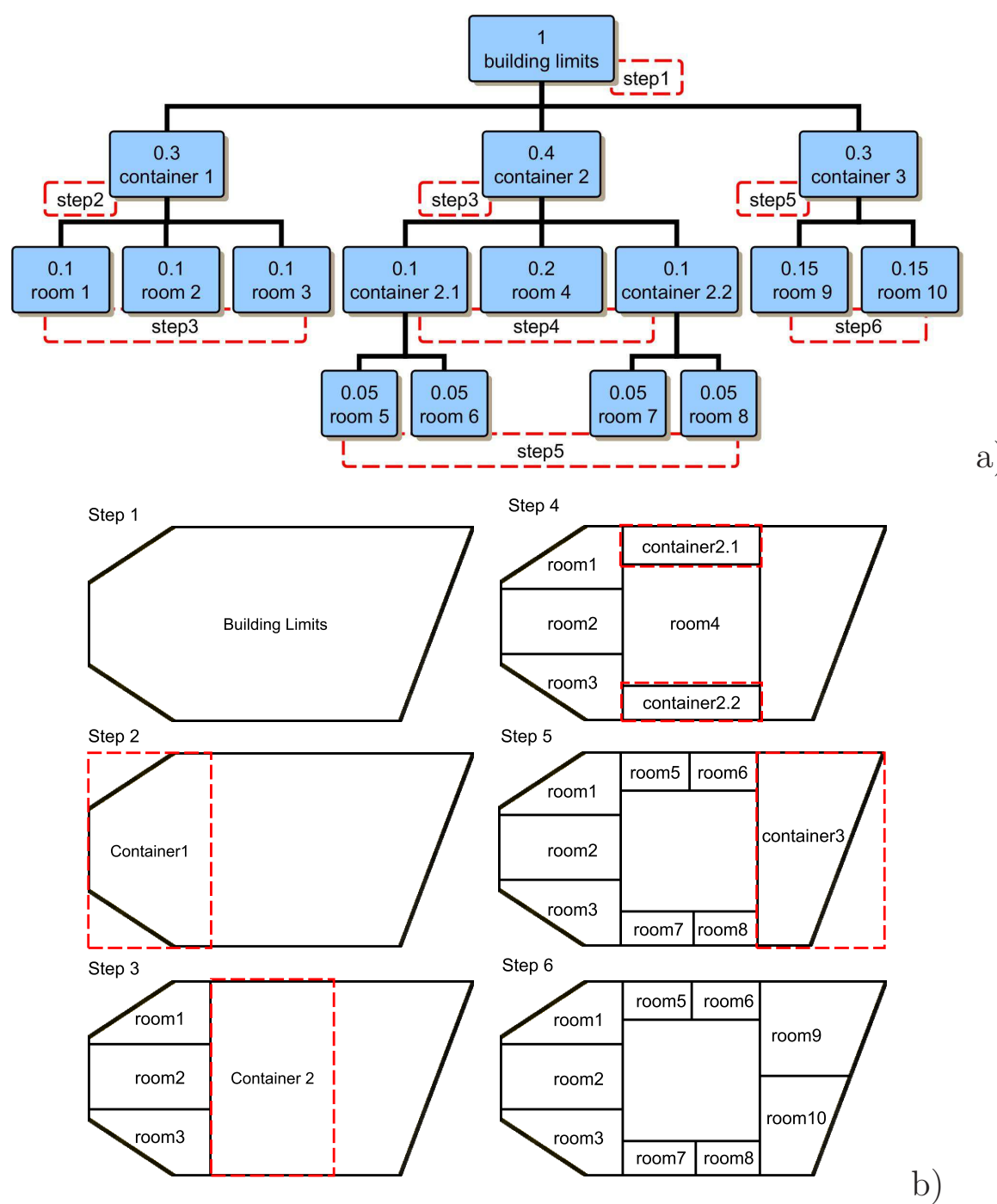

Figure 5.4 - Example of a treemap structure defining a floor plan arrangement, and respective floor plan subdivision process. (a) presents an example of a treemap structure defining a floor plan arrangement (explicitly provided by definition rules). (b) depicts the respective floor plan subdivision process based on the presented treemap definition. This schema depicts a set of steps representing the splitting process based on treemap. The first step is to find the total container which matches the outer limits. The second step places the container 1 and iteratively finds and adjusts the polygon that results from the intersection with the virtual building outer bounds, until the required occupation weight is reached. In the third step, the same process occurs inside container 2, but this time to place and expand final rooms (alias for divisions). In the remaining steps, the same process is applied until the final floor plan is obtained (sixth step). 


\subsection{Enhanced Methodology System Implementa- tion}

A system implementing the proposed enhanced procedural modelling methodology and capable of generating virtual traversable buildings constrained by arbitrary shapes, is presented in this section. Input definition rules are provided through XML4BD files that have a structure highly based on the designed building data model, previously addressed on subsection 5.1.1. Within the proposed system, there is a rule moderator module that implements the moderation process addressed in previous sections. This module checks both the XML4BD syntax and organization, loads the informations into a class structure that maps the ontology-based data model and filters inconsistencies, at the floor plan level, related with transitions placement. After proper validation, the previously loaded class structure is forwarded to the procedural modelling module which generates the virtual building, considering a set of default parameters - independent from the definition rules - that specifies architectonic constraints (e.g. wall thickness). The architecture of the proposed system is shown on Figure 5.5.

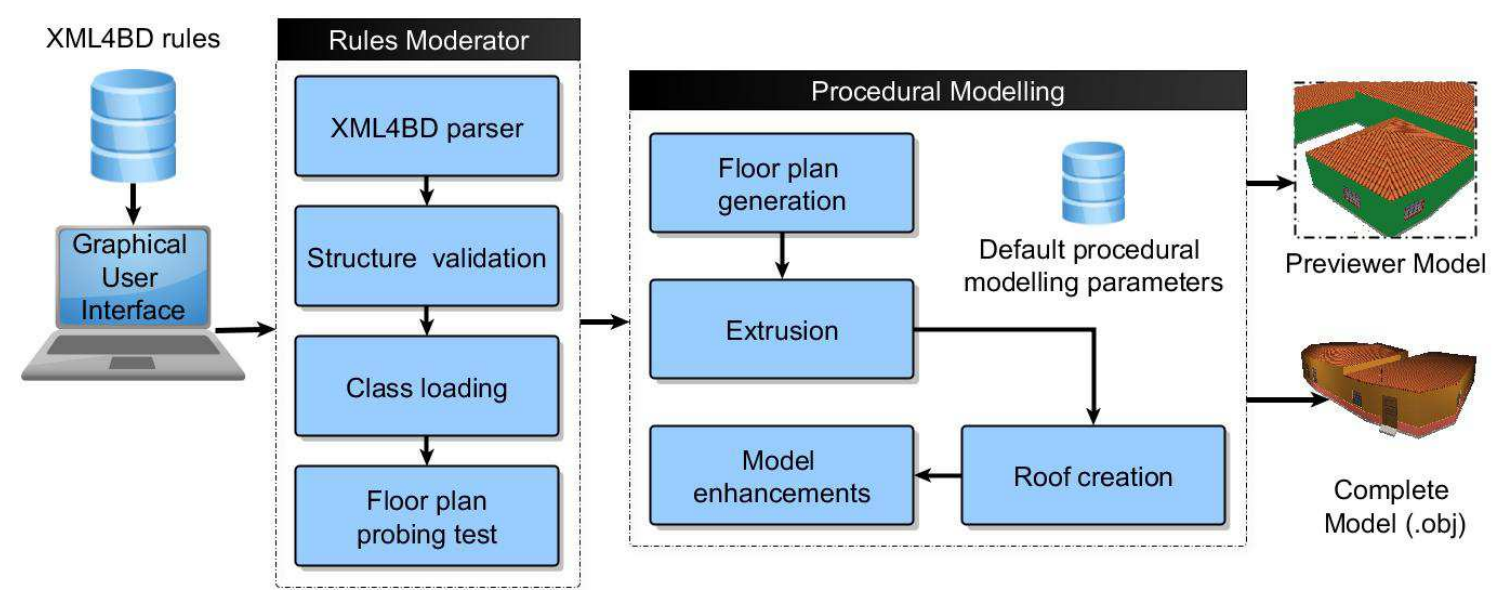

Figure 5.5 - Architecture of the system that implements the methodology for generating buildings constrained by arbitrary shapes, considering XML4BD specifications as input rules. The system contains a Rule Moderator module to validate XML input files and also a procedural modelling module to generate the geometry of the virtual building. 


\subsubsection{An ontology-based XML for virtual buildings defini- tion (XML4BD)}

The structure of the adopted XML format, designated XML4BD, follows the ontologybased data model structure depicted by Figure 5.6. Thus, inside the building node, it is defined the constraint polygon and building parts (horizontal compositions of the building) are defined. Inside each building part node a set of floors can be specified (although, the procedural modelling process only deals with buildings containing building parts with a single floor) and inside each floor node there is a group of divisions. Inside the virtual building node are also defined the connections between divisions. Each node that is part of the referred XML4BD structure is strictly related with the previously proposed data model (subsection 5.1.1) regarding both semantics and data structure.

The following XML sample represents the node system previously presented. The root is the building node. The geometry node defines the building contour which is given by a point set. Inside building node, a unique building part node is defined. It has a floor node which contains divisions. The first division node is a temporary container that arranges two other division nodes: final room and bathroom. At the same temporary container level there are two other division nodes defining a corridor and a kitchen. The transitions are provided in two distinct specifications: windows (maxWindows) and entry door (isEntry) are defined in each division node whereas the inner transitions are mapped in the adjacency node set which specifies the connectable divisions by relating identifiers.

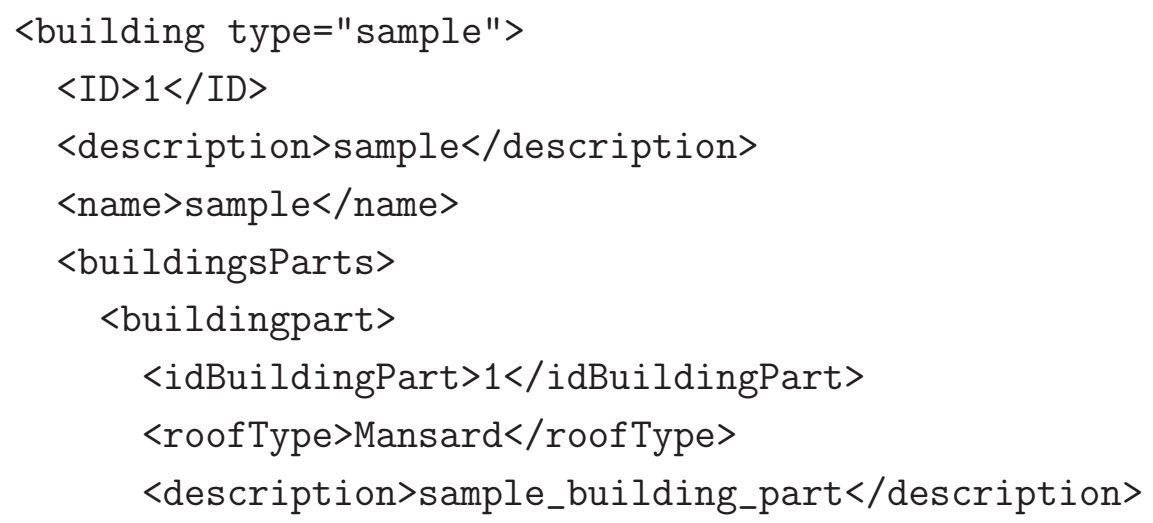




\begin{tabular}{|c|c|c|c|c|c|c|c|c|c|c|}
\hline \multicolumn{11}{|c|}{ Building } \\
\hline Geometry & \multicolumn{4}{|c|}{ BuildingParts } & \multicolumn{6}{|c|}{ Transitions } \\
\hline Points [...] & \multicolumn{4}{|c|}{ BuildingPart0 } & \multicolumn{2}{|c|}{ Adjacency } & \multicolumn{2}{|c|}{ Adjacency } & \multicolumn{2}{|c|}{ Adjacency } \\
\hline & \multicolumn{3}{|c|}{ Floor0BuildingPart0 } & RoofType: Hip & A & B & A & B & A & B \\
\hline & \multicolumn{4}{|c|}{ Divisions } & i & i & i & $\mathbf{i}$ & $\mathrm{i}$ & i \\
\hline & \multicolumn{2}{|c|}{ Division 0} & \multirow[t]{2}{*}{ Division1 } & Division2 & i & i & i & i & i & i \\
\hline & \multirow{2}{*}{\multicolumn{2}{|c|}{ Divisions }} & & & $\mathbf{i}$ & i & $\mathbf{i}$ & $\mathbf{i}$ & i & i \\
\hline & & & Attributes & Attributes & 0 & $\circ$ & 0 & 0 & 0 & 0 \\
\hline & & & & & 01 & 1 & 02 & 1 & 1 & 2 \\
\hline & Div.00 & Div.01 & & & & & & & & \\
\hline & \begin{tabular}{|c|} 
Attributes \\
{$[\ldots]$}
\end{tabular} & \begin{tabular}{|c|} 
Attributes \\
{$[\ldots]$}
\end{tabular} & & & & & & & & \\
\hline
\end{tabular}

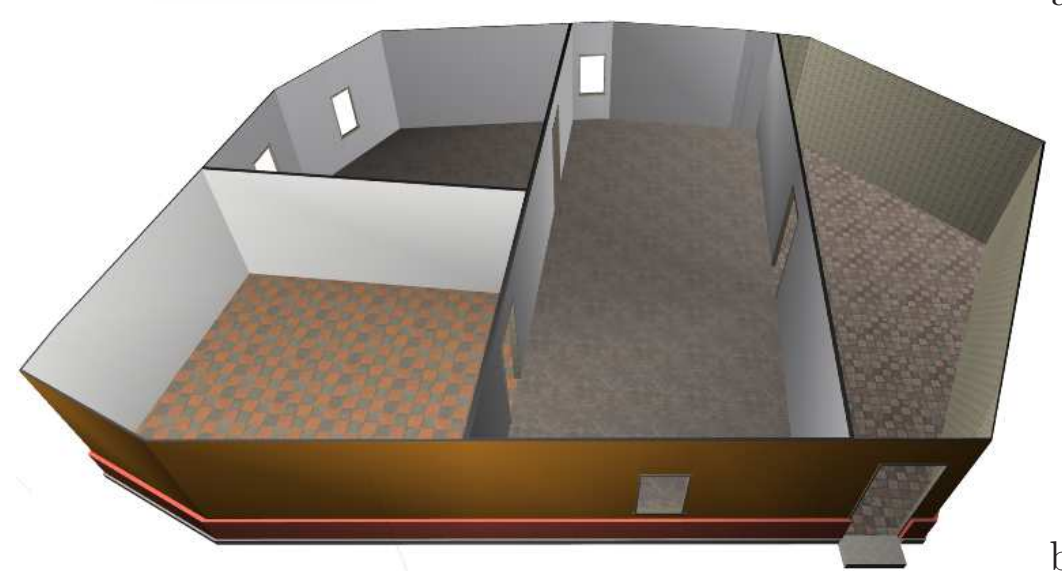

a)

b)

Figure 5.6 - Example of a XML4BD node system and resulting 3D virtual model. In a) an example of a XML4BD node system is depicted. The building root node has a nested buildingpart, a set of points representing the constraint polygon and a list of transitions (nested set of nodes specifying inner divisions' connections). That building part node contains a nested floor node - FloorOBuildingPart0 - with four divisions: besides final division Division1 and Division2, there is also a Division0 which is a temporary container for two other final divisions: Div.00 and Div.01. In b) the resulting virtual building, composed by one building part and four connecting divisions is shown.

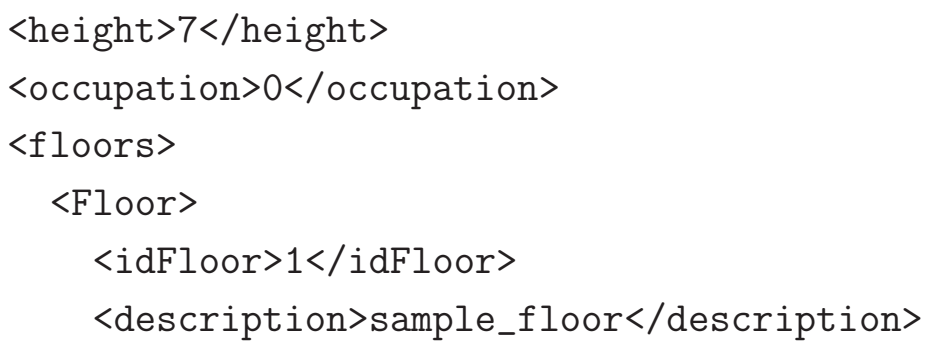




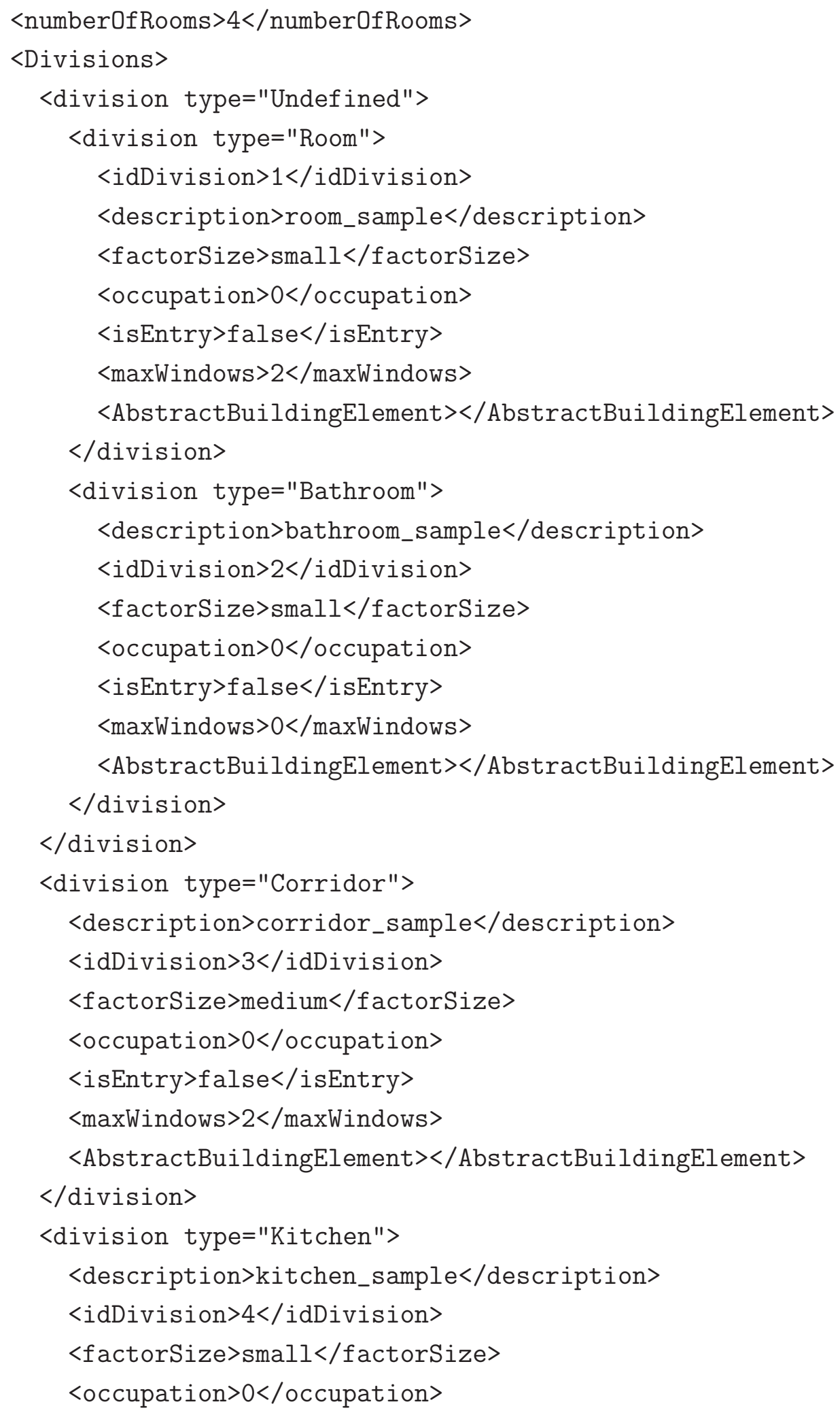




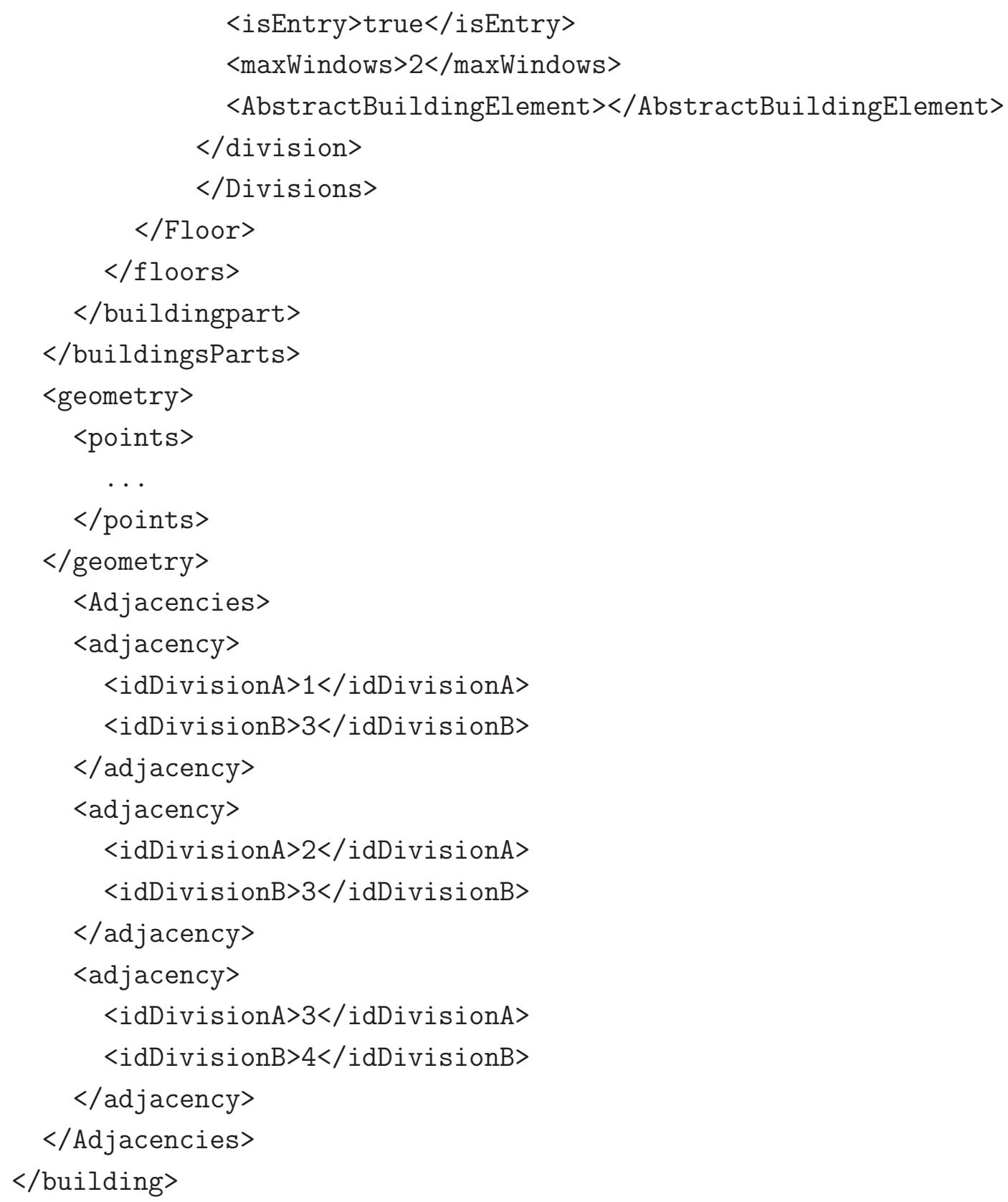

\subsubsection{Rules moderator module}

A rules moderator module incorporates this system to validate the XML4BD files (input definition rules) regarding its syntax and organization. This module is also responsible for loading an ontology-based class structure - implemented considering 
the proposed data model presented on subsection 5.1.1 - with the proper data. After that, floor plan probing takes place to check the transitions of connecting divisions: the ones that do not fit, are dismissed.

The process regarding rules moderation, presented in the previous subsection 5.1.4, is closely related with the steps performed by this module, as it intents to point the following items:

- Rules validation: initially, the input XML file is parsed to check inconsistencies on structure and writing. On the one hand, misplaced nodes are used as indicators of malformed structures. On the other hand, writing inconsistencies are filtered through the detection of unrecognised symbols or wrong specification types (for example, it is expected a numeric value for parameters that define the maximum number of windows per division). These errors are then properly returned to the user, stopping system's execution until new demands;

- Class loading: this step is reached when the input file is in full agreement with the ontology-based data model and there is no inconsistencies to overcome. Its execution aims the creation of the building instance as well as its composing elements in a class structure that faithfully maps the previously presented data model;

- Floor plan probing test: the instances of the resulting ontology-based class structure are subjected, by the rules moderator module, to some operations that have the goal of correcting instance parameters, specifically the ones regarding the floor plan generation. Thus, the execution of this task starts by testing if the connecting rooms belonging to a given building have sufficient space in their common wall segments to hold a transition. The pairs of connecting rooms that do not fulfil this requirement are provided with space from other rooms (available to borrow), in an attempt of making a larger adjacency for placing a transition. The ones that reach the borrow limit are reset to their starting sizes and their failing transitions are deleted to avoid further issues in the procedural generation module. 
After performing these steps, this module releases the class set to the procedural modelling module, addressed in the next subsection.

\subsubsection{Procedural modelling module}

The aim of this module is to semi-automatically generate virtual building models. Its operation is very similar to an automatic house-builder when constructing a given building by following the architectural plan orientations whereas in this case, information is provided through XML4BD definition files. Thus, the procedural modelling module uses the class structure previously loaded and duly verified by the rule moderator module to obtain virtual building's definitions. Among those classes, there is a set of mandatory data that includes the polygon that defines the virtual building limits, their constituting divisions and respective organization, their occupation weights in the restriction polygon and also the connections between them. Considering those definitions, this module starts a progressive process to produce a $3 \mathrm{D}$ virtual building model.

The first step regards the floor plan generation process and groups four operations: constraint polygon determination (must be convex, otherwise the module forces this requirement using a convex hull algorithm), floor plan area division (according with the loaded class structure, based on XML4BD rules), placement of external doors and windows and lastly the placement of connecting doors between divisions (correspondent to the graph defined in class structure, loaded from XML4BD input file). The walls' extrusion step will raise the walls in the virtual building, considering its transitions. The few last steps will generate grounds, ceilings and roof, thus completing the $3 \mathrm{D}$ virtual building model.

Some tests involving ancient structures were made to demonstrate the effectiveness of the developed system while applying the procedural modelling methodology to generate virtual buildings constrained by convex shapes. The next sections focus these tests along with the achieved results. 


\subsection{Preliminary Tests and Results}

The first trials consisted in generating missing ancient buildings based on textual descriptions (Adão et al., 2013a). After analysing the available texts provided in Conimbriga's website (Correia, 2002), a set of ontology-based XML files (a prerelease of XML4BD) were set up with the most relevant information found in those on-line documents (e.g. restriction polygon and division arrangements). Then, these files were submitted to the procedural modelling system to generate the complete geometry of the buildings, including its exteriors and interiors. Results are presented on table 5.1

Table 5.1 - Reconstructed roman buildings of Conimbriga (Portugal): the first exposes the house of skeletons; the second shows the reconstruction results of the house of fountains; the third presents via south stores; finally, the last depicts the reconstruction results of the house designated by insula aqueduct.

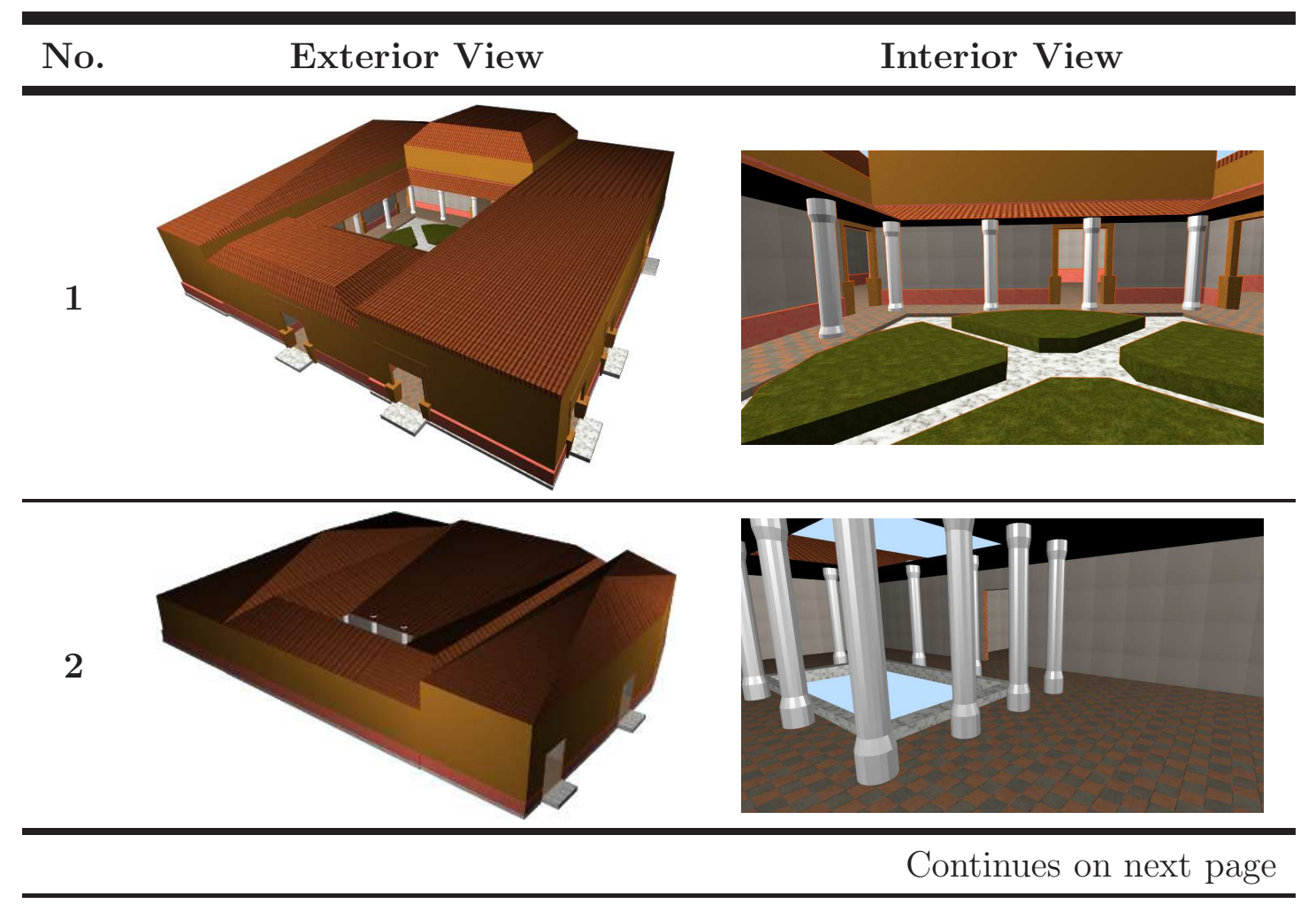


Table 5.1 - continued from previous page

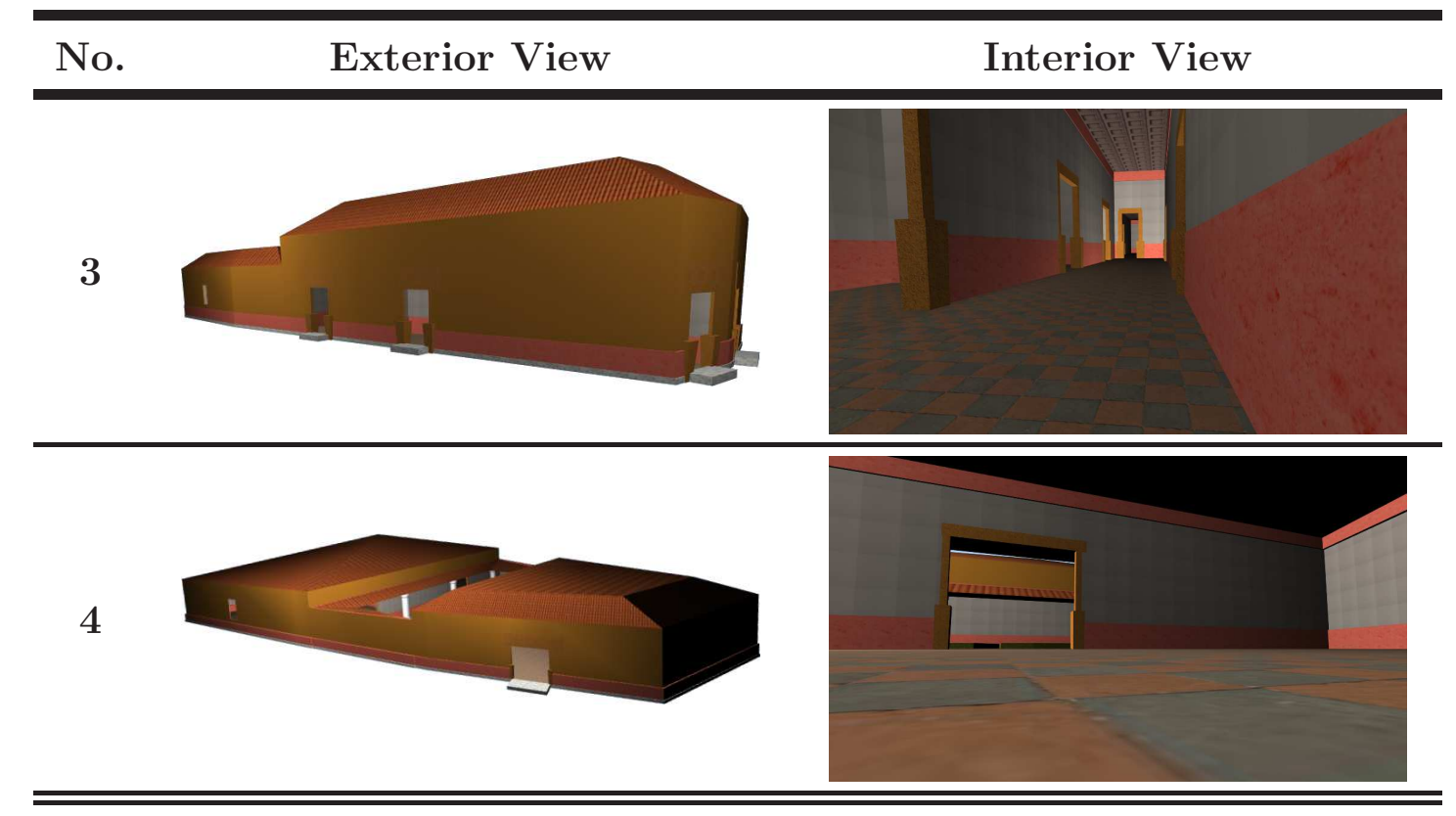

A few pilot experiments were made (besides the previously addressed test to reconstruct missing buildings) to demonstrate the capabilities of the procedural modelling methodology system in generating traversable buildings constrained by arbitrary shapes. A set of XML4BD files were populated with data to simulate fictitious ancient structures. These buildings were projected to be outlined by arbitrary convex polygons. Each building has different configurations, with distinct building parts, divisions, size factors, arrangements, connections and structures. The involved complexity ranges between a simple building with four inner divisions and a more complex edifice having several divisions and an inner garden. Each XML4BD file is provided as input to the system and validated by the rules moderator module, which results in an ontology-based class structure properly loaded with building's data. These classes are then processed by procedural modelling module, which produces the 3D virtual building. The verified XML files tested followed the format addressed on section 5.2.1. 
Specifically, three buildings were defined accordingly with the XML4BD format. All of them are constrained by different convex polygons with variable dimensions. The first building is the simplest one. It includes a kitchen - the access division (or room) of the building - which is connected to a hall that conducts to a bedroom and also a bathroom. Then, a second building is presented with an entry kitchen connected to a living room which also contains a passage to a wider generic room. Generic room interfaces with a bedroom and also a bathroom. Finally, the last building includes five rooms (final divisions), a kitchen and a bathroom. At the center, there's a division that holds a special structure: a garden with four plant areas and also columns. The garden division connects all other division. Table 5.2 shows the graphical results of the XML4BD files processing.

Table 5.2 - Ficticious virtual building models representing ancient roman structures and produced by the system that implements the methodology for generating buildings constrained by convex shapes. three different XML4BD files were defined to trigger the generation of the following buildings: two simple houses with four and five divisions, respectively and a third house with a eight divisions, including one that contains a central garden.

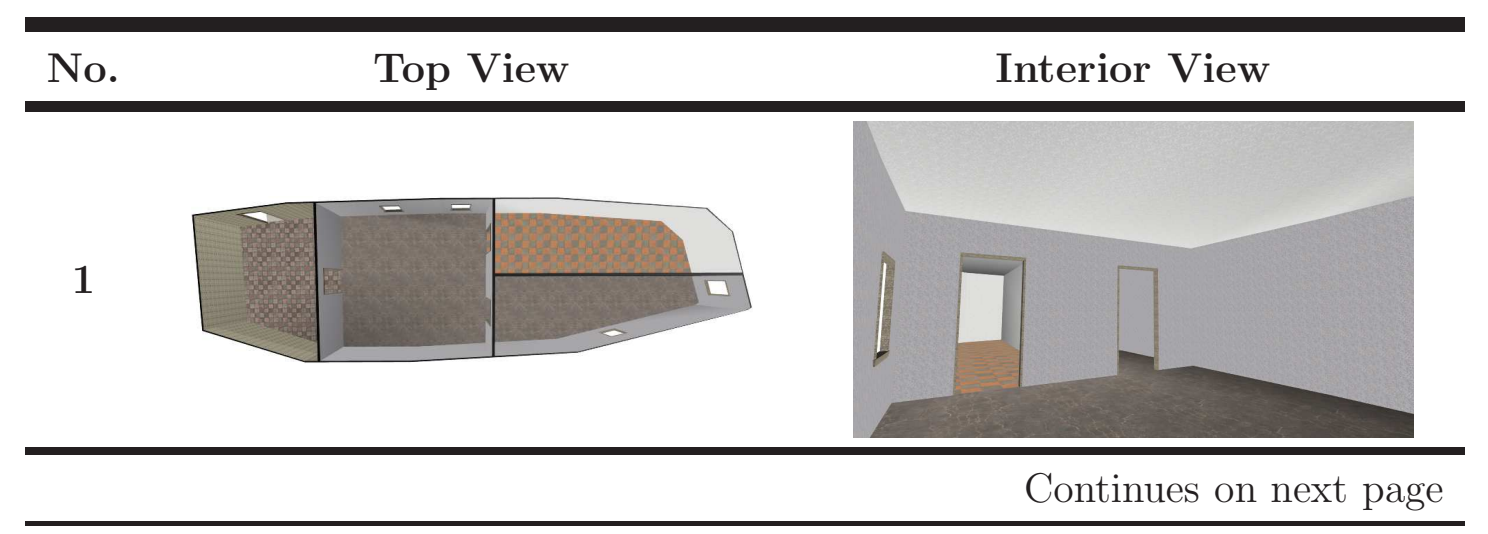


Table 5.2 - continued from previous page

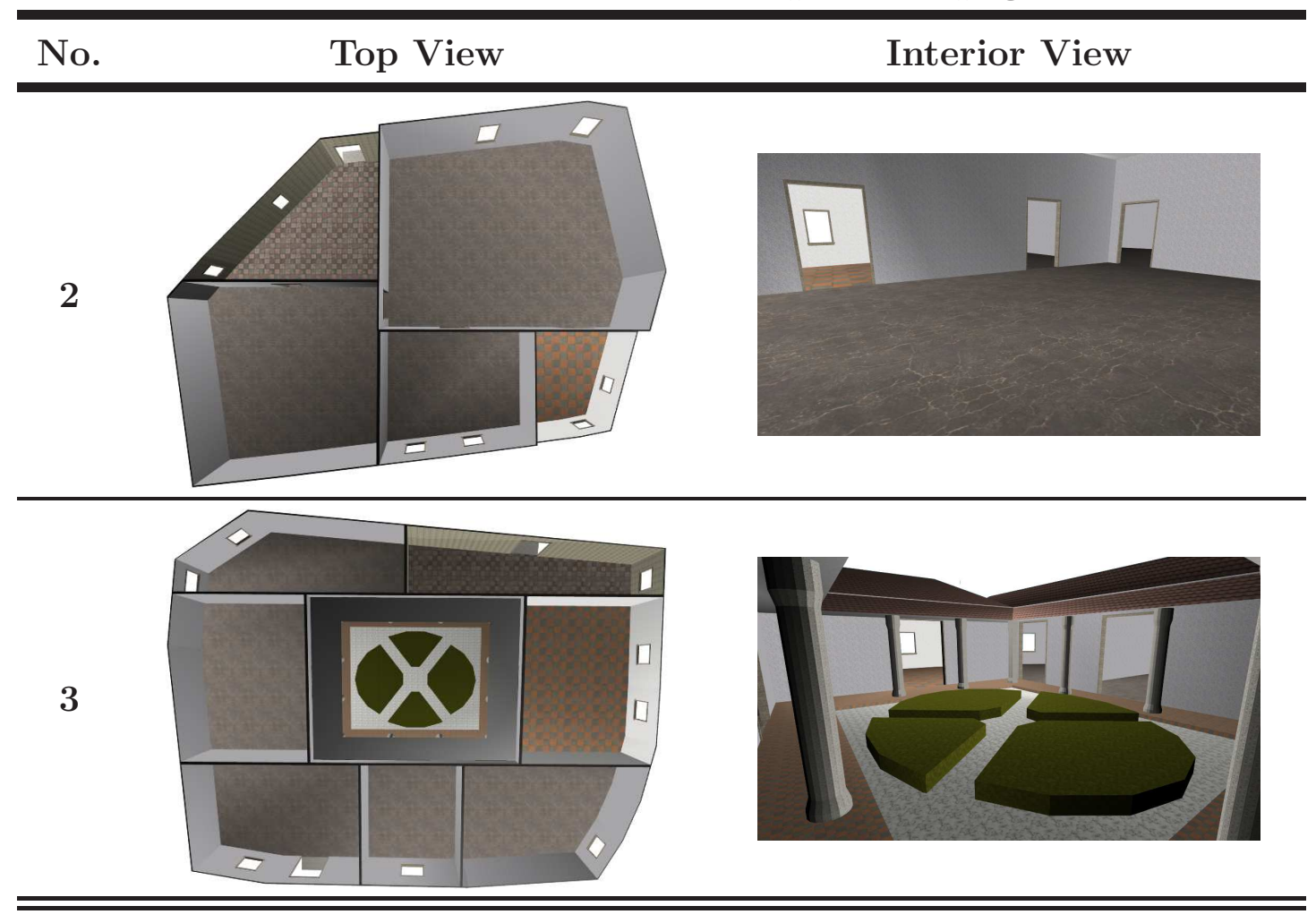

\subsection{Summary}

This chapter presented the enhanced version of the proposed procedural modelling methodology which, at this point, is capable of producing 3D traversable buildings constrained by convex polygons. A rule moderation process was also proposed to filter syntax and structuring errors in rules. Moreover, this moderation process also analysis divisions' connectivity with floor plan probing tests that enable to discard geometrically unsupported transitions and avoid errors in further procedural generation stages. The module result is a clean ontology-based class which is then converted into a virtual building by the procedural modelling process. The floor plan generation is the first stage and employs some operations to deal with convex constraint shapes delimiting the building. Lastly, the complete virtual 3D building 
model is achieved through a set of extrusion and enhancements (e.g. transitions' frames).

A system was implemented to be used as methodology's proof of concept. The system receives as input an ontology-based XML - also known as XML4BD - which contains the definitions of a given building, including its related building parts, floors, divisions and transitions. The file passes through a XML4BD (rules) moderator module that reads and validates the XML4BD structure. The referred moderator also loads an ontology-based class structure and performs a few tests to ensure the coherency related with the floor plan (e.g. elimination of transitions between divisions that do not share a common wall). Then, the action of the procedural modelling module takes place. This module iterates through the class structure to produce 3D virtual models, from the floor plan step until the last model enhancements.

The performed tests to the system demonstrated the versatility of this tool in creating buildings with several configurations and topologies. Results pointed out that this tool is effective in producing buildings constrained by pure convex shapes. Besides, it is also possible to include in the building special divisions holding inner structures, like pools or gardens with columns.

This methodology has still, however, some limitations: the generation of floor plans does not support holes (absence of geometry) and the division's shapes cannot be altered. The next chapter proposes the final procedural modelling methodology, by addressing these issues. Moreover, a stochastic approach for the random generation of buildings is proposed as an experimental attempt of automating the methodology. 



\section{Generation of Virtual Buildings Composed by Arbitrary Shapes}

The current version of the proposed procedural modelling methodology extends from the one presented on chapter 5, which uses a set of ontology-based rules, inferred from a data model designed in concordance with the ontology presented on chapter 3 , to guide the generation of ancient virtual buildings, delimited by non-convex shapes. In this chapter, the final version of the procedural modelling methodology is presented. This new version has some enhancements worth noting: the aforementioned rules evolved to an ontology-based grammar; the procedural modelling process is improved to support the generation of virtual buildings delimited by nonconvex shapes, with divisions constrained by a variable number of delimiting wall segments; finally, a stochastic process is proposed to have a fully automatic generation of virtual building models, with minimal user inputs. Specifically, two types of stochastic requests can be made with a proper input set: for an expeditious generation of a random buildings' set, the desired number of buildings has to be provided; alternatively, to generate a building with a specific divisions set and configuration, a list enumerating those divisions has to be submitted along with the desired building configuration (L-shaped, U-shaped or fully convex). For each generation task, the proposed stochastic process arranges the rooms (or divisions) by priorities, mounts the graph of connecting divisions and randomly arranges them in a node structure that mimics a treemap. Therefore, this node structure is then transformed into 
grammar rules that are used to properly produce a 3D virtual building model, using the final procedural modelling process.

A procedural generation system was also developed to implement the proposed procedural modelling methodology in its final version. Within this system, two tools deterministic and stochastic - were developed. The former relies on user floor plans drawings alongside with its parametrization. The latter permits the expeditious generation of random virtual buildings through computer-managed selection, based on two possible types of user inputs: a restrictive list of divisions and building configuration or the desired number of buildings to be generated. As a result, both tools produce a set of ontology-based grammar rules (or consecutive sets, in the case of user request for random buildings production through the stochastic tool). Then, those rules pass through the couple of system's modules to be properly processed. After grammar rules validation and floor plan probing (pre-adjustment trial to ensure the connectivity between divisions and/or discard useless transitions), a sequence of steps is performed to achieve the 3D virtual model. The first step regards the floor plan generation, where the building's constraint polygon is recursively divided to create division areas. After defining all of the division areas, the number of wall segments is adjusted accordingly with the grammar rules requirements, which is also an enhancement in this final version of the proposed methodology. The next step is to place transitions between divisions. Afterwards, the extrusion is applied to both interior and exterior walls and, finally, the roof, division ceilings and grounds placement occurs. Resulting buildings may be outlined by non-convex shapes through the application of a "fake-concave" technique in the aforementioned floor plan subdivision step.

This chapter is organized as follows: in the first section, the final version of the procedural modelling methodology is presented, including some enhancements regarding the previous one such as the generation of floor plans delimited by non-convex polygons, the adaptation of interior divisions' walls and the stochastic generation of virtual buildings; next, the system that implements the proposed methodology through both a deterministic and a stochastic tools is presented. Preliminary tests focusing both the methodology's capabilities and the stochastic process flexibility 
are presented in section 6.3 .

\subsection{Final Procedural Modelling Methodology Pro- posal}

In chapters 3 and 4 , the treemap approach was suggested to generate virtual buildings composed by rectangular shapes and constrained by convex limits, respectively. Some enhancements made to these versions led to the final procedural modelling methodology version in which a process for producing virtual traversable 3D building models composed by a single floor and constrained by arbitrary shapes is proposed. The ontology proposed on chapter 3 is still used for regulation purposes.

Based on the aforementioned ontology, a grammar is also proposed in this chapter, establishing the latest form of definition rules for buildings. Each set of these rules consists in a per-building specification in which the treemap of building parts and divisions is defined, as well as transitions and other relevant features like divisions' occupation weight and building parts' heights. Moreover, a new rule type that enables the specification of disposable building parts integrates this ontology-based definition rules. Such rule type is applied in the floor plan generation stage to delete building parts marked as disposable, as it will be explained later. Then, a verification stage - similar to the one presented on chapter 5 - debugs the grammarbased definition rules, regarding structure and typos. After validation, these rules are used to properly load an ontology-based class structure which is then subjected to a probing stage to ensure the feasibility of the floor plan. The probing stage consists in balancing division's occupation weights to make room for transitions or dispose the ones that cannot be placed between connecting divisions (already addressed on chapter 5). At this point, the class set is ready to be submitted to the procedural modelling process that aims to produce the final 3D building model.

The aforementioned process occurs just like it was explained in the previous chapters. The initial goal of this process is the floor plan generation and the first step for this accomplishment consists in dividing the building's convex polygon into building 
parts - effective or disposable - and divisions, through a recursive treemap approach and considering the input definition rules. For each building part a floor is created and then subdivided into divisions. At the end of the building's polygon division step, the building parts marked as disposable are removed from the floor plan on which the following transformations may occur:

- if all of the removed building parts overlap the floor plan's constraint polygon (the same as building's constraint polygon), such polygon becomes non-convex;

- otherwise, holes are created somewhere in the middle of the floor plan, i.e., the floor plan is set to have "dead" areas without any geometrical definition;

- the excluded middle event results in a floor plan with holes and constrained by a non-convex polygon.

This technique was named as "fake-concave" and uses a labelling approach based on BSP trees to define disposable elements. A new step is introduced in this procedural modelling process before the transitions markings: wall segments are adjusted to inner divisions, considering the definition rules provided through the grammar and associated parameters. The next step is to mark doors, windows and to connect divisions. Afterwards, external access doors and windows are marked in the proper divisions, more precisely in the division walls' segments that are in contact with the building limits. Then, the doors are also marked between overlapping wall segments of connecting divisions. Subsequently, walls are extruded considering the previously marked transitions. Those transitions also assume a 3D look with frames properly adapted to fit doors or windows. Ceilings and grounds are also created and next roofs for each building part are generated accordingly with the type indicated on the grammar. Finally, the virtual model is completed with some enhancements such as division footers, roof skeleton coarsening and stair-steps for entry doors. 


\subsubsection{Ontology-based Grammar}

The previously settled definition rules (chapter 5) resulted from a data model containing a set of essential fields that aimed to characterize a building. In this section, a grammar was designed to define virtual buildings, considering the works of many other authors who decided to use grammars in procedural modelling (Rau-Chaplin et al., 1996; Wonka et al., 2003; Larive and Gaildrat, 2006; Müller et al., 2006b; Rodrigues et al., 2008c; Hohmann et al., 2010; Trescak et al., 2010). This grammar is highly based on the proposed building's ontology, even regarding the structure, making it different from the one proposed on chapter 4 .

The semantic scheme defined by the ontology constitutes the first regulatory structure for the presented procedural modelling methodology, creating the awareness of a generic building composition: buildings parts per building, floors per building parts, divisions per floor and related transitions such as doors and windows. This structure provides the possibility of defining a regulated sequence of operations and also the geometric transformations according to each element, while maintaining the coherence of the virtual model (see chapter 3 for more details). The referred ontology was extended with some fields - regarding building's information - aiming the elaboration of an ontology-based grammar.

The ontology-based grammar definition recovers two concepts used on chapter 4: Lsystem (Lindenmayer, 1968) and treemap (Johnson and Shneiderman, 1991). The L-System is used to iteratively produce transformations through the symbols which are context-free. However, transformations made to the symbols are "constructor functions" designed to operate considering ontology conventions. These transformations are also known as production rules and follow a logic sequence to achieve the building definition which comprises the number of vertical building parts and related floors, the divisions contained by each floor and also the connections between these divisions. Considering those structures a treemap of ontology-based objects is progressively mounted.

Everything starts with the building axiom, which is decomposed in building parts and transitions. Each building part is decomposed in floors and floors are then 
subdivided in divisions. Divisions and transitions are final symbols, with no decomposition. The referred production rules follow the format:

axiom->function(_parameters): \{set_of_resulting_symbols\},

where axiom is the symbol under processing, the function is the ontology based operation and the set_of_resulting_symbols constitutes the resulting symbols that should be processed in later steps. The ontology-based grammar functions are presented next.

\section{A) Building}

This function, defines a building's constraint polygon. The resulting derivations include building parts and transitions:

- building(size(x,y,z)): assigns a rectangular area for the building;

- building(list(point $(\mathrm{x}, \mathrm{y}, \mathrm{z})$, point $(\mathrm{x}, \mathrm{y}, \mathrm{z}), \ldots))$ : defines a building with a constraint polygon. The constraint polygon is a parameter expecting a set of points;

- building(generate_hull(num_points, width_min, width_max, aspect_ratio_min, aspect_ratio_max)): defines a building constrained by a random polygon, generated with a number of cloud points, minimum and maximum width and also a minimum and maximum aspect ratio (used to determine the buildings length).

The resulting symbols are a set of building parts and inner transitions to connect divisions.

B) BuildingPart

The buildingPart function defines containers for space arrangement purposes or horizontal building compositions. The use of this function produces two types of derivations: on one hand containers produce another set of axioms defining subbuildingparts while, on the other hand, final horizontal compositions originate the floor axiom: 
- buildingpart(roof_keyword, height_float): is used to define a buildingpart where roofkeyword defines the roof type that will cover the building part (possibilities: Flat | Pyramide | PyramideHip | Hip | Mansard | Combination | Gable | PorchHoled | None | MansardHoled), the height_float establishes the height of the building part. A building part with a height of 0 , is a hole on the building (useful to create, for example, L or U-shaped building formats). The resulting symbols are related with floor definitions;

- buildingpart(): used to define a container for other building parts, changing the axis of generation. If the building part is associated to a horizontal subdivision (x-axis), then its children should be divided following a vertical composition (z-axis) and vice-versa. This function returns a set of symbols related with sub-building parts.

C) Floor

Function regarding the floor definition requires the specification of the height for both the floor base and a secondary exterior wall. This function derives symbols for inner divisions.

- floor(base_height, exterior_wall_height): defines a floor with an exterior base and a secondary exterior wall. It retrieves a set of division symbols.

\section{D) Division}

Similarly to building parts, it is also possible to create division containers and final divisions (rooms) using division functions:

- division(num_wall_segments, size, division_type, special_structure, is_entry, num_windows, footer_height, topunion_height): this function declares a division with a fixed number of wall segments (if num_wall_segments is lesser than 4, the number of wall segments will remain unchanged which means that the container shape and also the adjacent divisions will define the number of wall segments of this division; otherwise, division's wall segments will be added or deleted in order to fulfil the requirements of the input parameter), a certain size (small | medium | big), a division type (room $\mid$ corridor $\mid$ 
kitchen | toilet | hole), special structure (garden [supported by the generic ontology] | pool [supported by both the generic and the roman ontology] | none), a boolean specifying if a division should have a transition to the exterior and a height value for the bottom footer and for the header ceiling junction. It does not produce any axioms;

- division(): with this function, it is possible to create subdivisions inside a division, just like it is done with building parts. The function should retrieve a set of axioms for its child divisions.

E) Transitions: this function defines the graph of inner divisions, i.e., provides a way of specifying the connectivity between divisions in order to ensure the circulation inside the virtual building:

- transitions (list(transition $(\mathrm{d} 1, \mathrm{~d} 2), \ldots))$ : it specifies the connections between the divisions belonging to the virtual building. This function works upon a final axiom defining the list of divisions that should be connected in the building.

\subsubsection{Moderating ontology-based grammar rules}

The moderation process proposed in chapter 5 was adapted to fit this ontologybased grammar format. Firstly, each grammar-based instruction set is verified to check typos (unknown syntax) and structure (sequence conformity). Then, a floor plan probing is performed to ensure that the weights and the divisions arrangements are properly set up, using the same operations addressed in the moderation process presented in chapter 5. Thus, connecting divisions are preliminarily tested with the placement of transitions upon overlapping divisions' wall segments. Those that do not fit trigger weight borrowing process among divisions. When space collection reaches the limit - provided by the restriction rules specified on chapter 5 - in a given couple of connected divisions, one of two events might happen: if the transition fits 
it is considered valid; otherwise, the transition is discarded to avoid malformed geometries in further operations, specifically those regarding procedural modelling.

\subsubsection{Procedural modelling generation process}

The procedural modelling process starts by considering the properly validated virtual building definitions, provided by a set of grammar-based definition rules in order to obtain its working requirements. The mandatory data provided by this set includes the polygon that defines the building's limits, the virtual building constituting divisions and respective spatial organization, the weights of occupation of each division in the restriction polygon and the connections between them.

Once the floor plan definition is determined, a progressive process is triggered to produce the virtual building model. The initial steps are common to the ones described in previous procedural modelling methodologies versions: firstly, the restriction polygon is determined and forced to be convex; in the next step, the floor plan subdivision takes place to divide the virtual building polygon into smaller areas, according to grammar specifications.

At the rules definition time, building parts can be marked as empty - height equals to 0 - which means that they will be disposed after the floor plan division. This results in a geometric hole that changes the contour shape that constraints the virtual building. This "fake-concave" technique, depicted in Figure 6.1, relies on the BSP trees labelling approach to define disposable building parts.

A new step is introduced in the procedural modelling generation process before the placement of transitions: shape adaptation for inner divisions. This step accomplishes the transformation of the division's shape to fulfil the requirement that defines the number of bounding wall segments. Therefore, some segments may be added or deleted from the shape accordingly with the parameter that sets the divisions' wall segments. Note that at this stage, the floor plan is in 2D (ground level) and these segments represent non-extruded walls, i.e., they are only 2D ground marks for a future extrusion. 


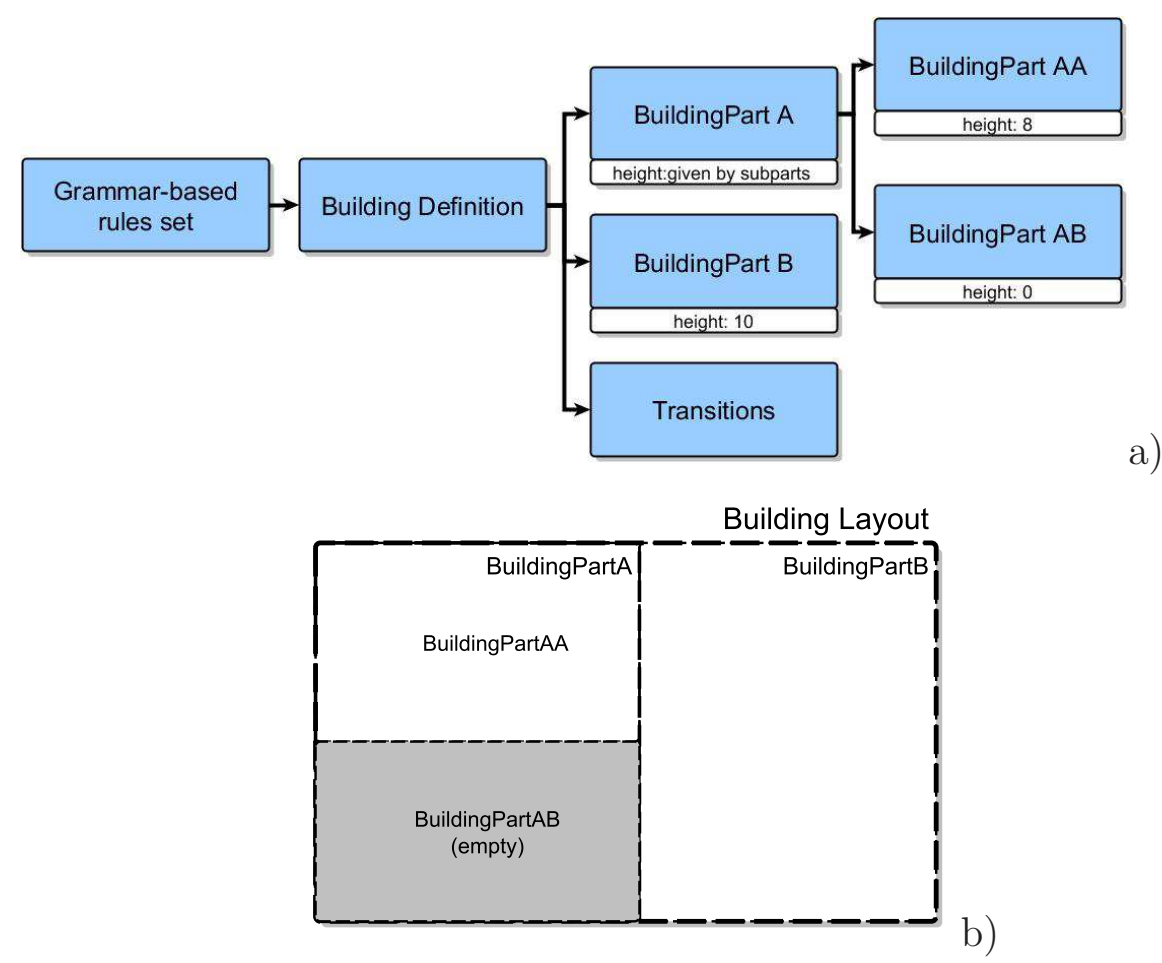

Figure 6.1 - Example of the fake-concave technique behaviour. In a) is a graphical interpretation of the building parts specification (provided by definition rules). Each one of those building parts can be defined as a geometric hole if the height is set to 0 . b) presents the expected result obtained from previous definition. BuildingPart $A B$ is a geometric hole because its height was set to 0 , at the definition time.

The first grammar rules parameter that is related with the division definition establishes the number of wall segments that will delimit each division (a wall segment is defined by two consecutive points, before the extrusion step). This parameter is considered at the procedural modelling stage, after the division process. Thus, when the process of dividing the building's constraint polygon is complete - through the splitting operations guided by the treemap implicitly defined in rules - the resulting divisions are geometrically moulded by the exterior walls, while the wall segments that constitute a frontier with adjacent divisions maintain their format (which was given by the initial splitting area created during the floor plan division stage). To match the number of each division's wall segments with the correspondent wall segments parameter value specified by the definition rules, some adjustments might 
have to occur, which means that some wall segments need to be added or subtracted to divisions that do not comply with the rules regarding the number of wall segments. These two adjustment processes are now described in grater detail.

- Adding wall segments to a division: while the number of wall segments belonging to a given division is lesser than the value defined in the rules, some operations have to be iteratively performed to increase the effective number of wall segments. These operations consist in testing and finding the most longer segment to be replaced by a certain point, avoiding interfering with inner transitions. Thereby, at the beginning of the process, a simulation to place those transitions is performed (preliminary step with no effective changes). Afterwards, each point of the division shape (formed by its segment walls) is subjected to an isolated simulation (for a given iteration, all other points remain in the shape while a particular point is exclusively tested). Each simulation corresponds to a point iteration consisting in the replacement of that point by a segment, properly spaced between its next point and its previous point. As long as that segment does not collide with transition marks, the length of the replacing segment is calculated and stored along with the testing point in a dynamic structure. Otherwise, the segment is ignored and the process continues to the next point. After testing all points belonging to the division's shape, the aforementioned structure is traversed to check the segment with the greater length and the effective replacement occurs in the associated point. The process is repeated until the effective number of wall segments comply with the rules. The wall segments addition workflow is presented in the Figure 6.2.

- Deleting wall segments from a division: alternatively, if a given division exceeds the required number of wall segments - specified by the rules - then one or more segments need to be deleted without interfering with transitions. Thus, initially, a simulation is performed to place the division's transitions, temporarily (preliminary step with no effective changes). The set of points constituting the shape formed by the division's wall segments are subjected to a test that aims to find the candidate point for exclusion, involving the minimal division area loss. Thereby, for each point, the following set of operations 


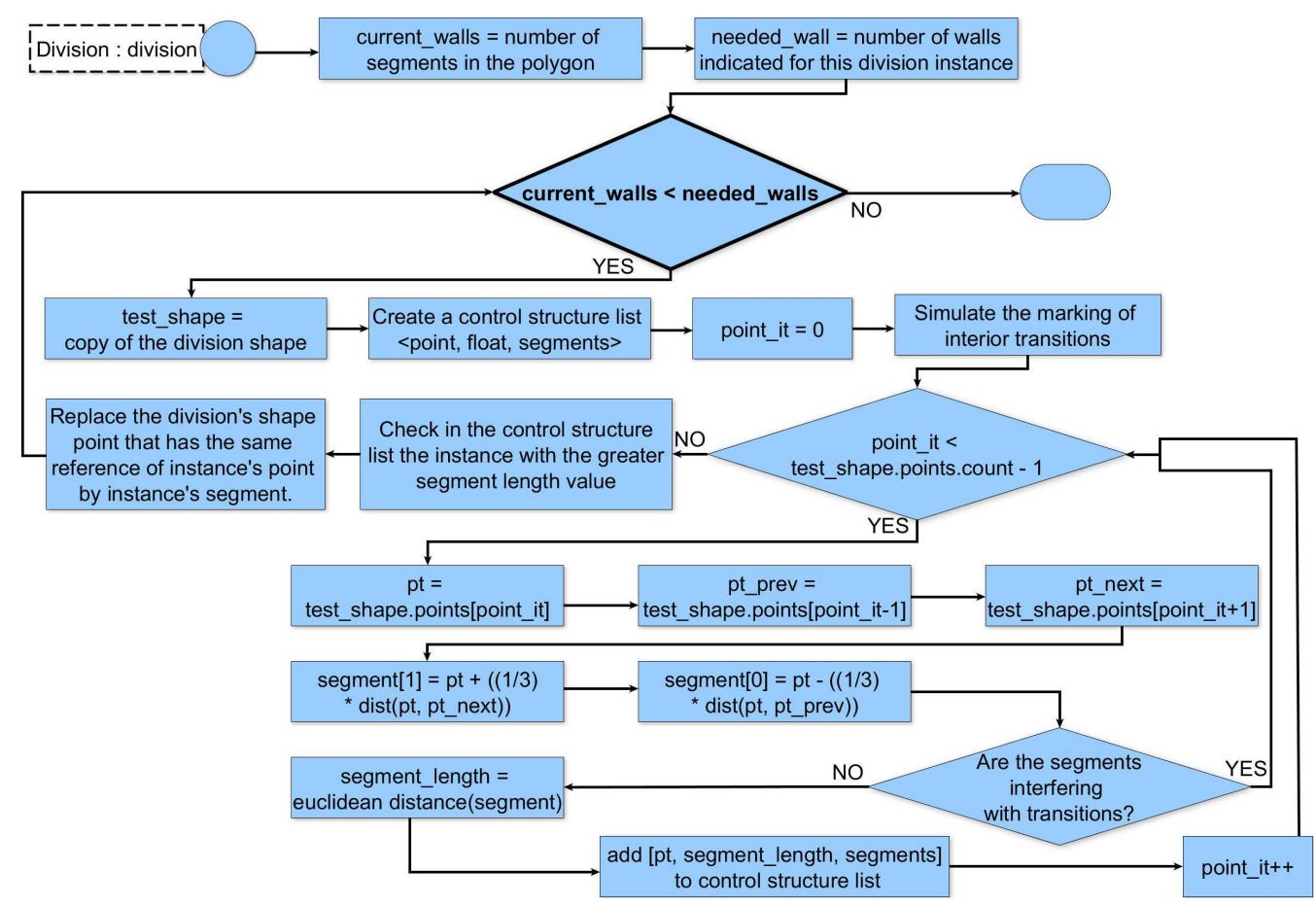

Figure 6.2 - Workflow depicting the addition of division wall segments. Wall segments are added to the existing division wall, while the number of division wall segments is lesser than required. Each iteration avoids interfering with interior transitions and uses the most lengthy segment to increase the wall, which aims a balanced distribution of segments throughout the wall.

are carried out: firstly, the point is selected; then, the point is temporarily removed from the aforementioned shape; a verification is also made to ascertain if there are any transition affected by that removal; if so, the current point is ignored and the next one is processed from the first step; otherwise, the process continues with shape's area being calculated and associated to this point; finally, the removed point is restored. When the testing process is ended for all points, the one having the associated higher area value is effectively removed, reflecting this change in the wall segments. The process is applied while the number of wall segments is greater than the specified by definition rules seeking, in each iteration, the preservation of the widest division's area as possible. Figure 6.3 depicts the general workflow for the division's wall segments removal. 


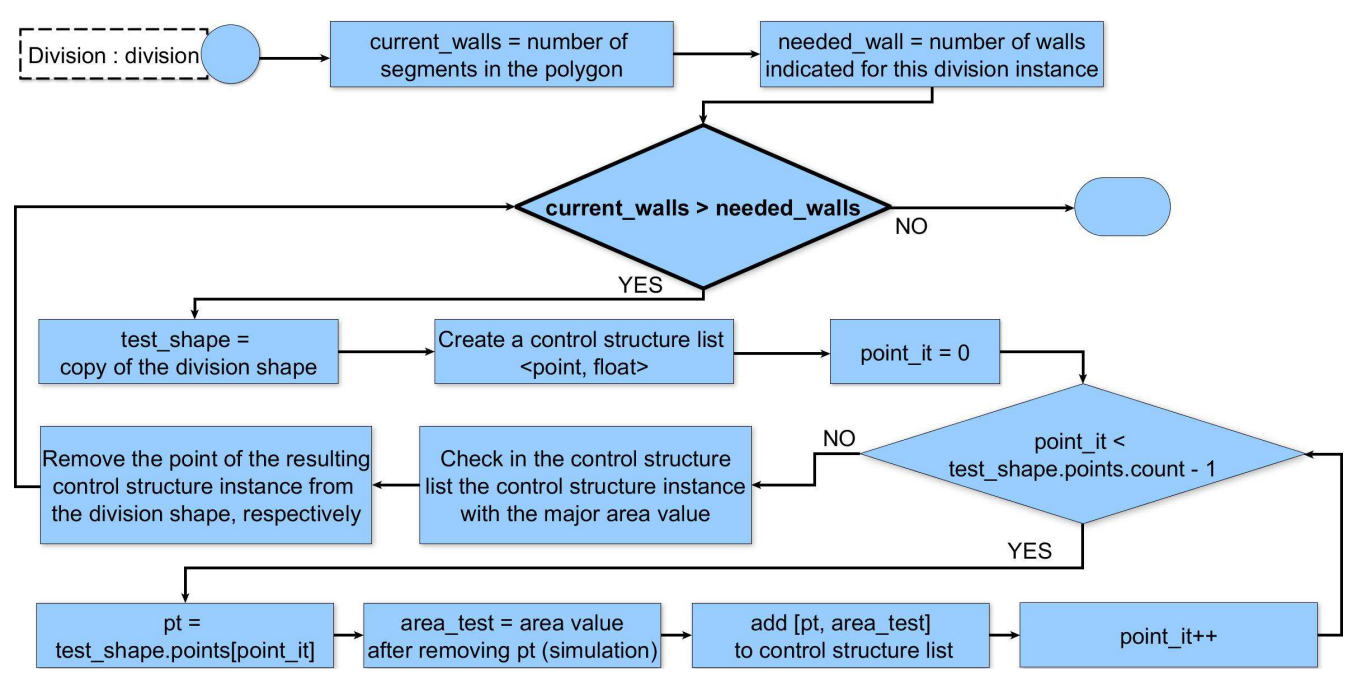

Figure 6.3 - Workflow depicting the operations regarding the deletion of a division's wall segments. While the number of wall segments is higher than required, wall segments are taken out (through point elimination) in an operation that ensures the minimal waste of a division's area.

A schematic example of the operations performed when adding or deleting wall segments is presented on Figure 6.4. Point replacement by segment operation is depicted on Figure 6.4 (a). The point that will be used for the effective replacement is selected accordingly with the most lengthy segment determined through simulation, as it was previously explained. The process also avoids to apply the replacement on segments that interfere with transitions, in order to preserve the connectivity between divisions (required by rules). On the other hand, the process of point exclusion is shown in Figure 6.4 (b) and loops until a match between the number of division wall segments and the number of wall segments specified by rules occurs. Such process ensures a minimum area loss regarding the division's shape being processed.

The process of division walls adaptation (addition or deletion of points) also relies in the following assumptions:

- The maximum number of division wall segments is 8 . When the rules provide a higher value than 8 , the value 8 is considered instead of the defined. 


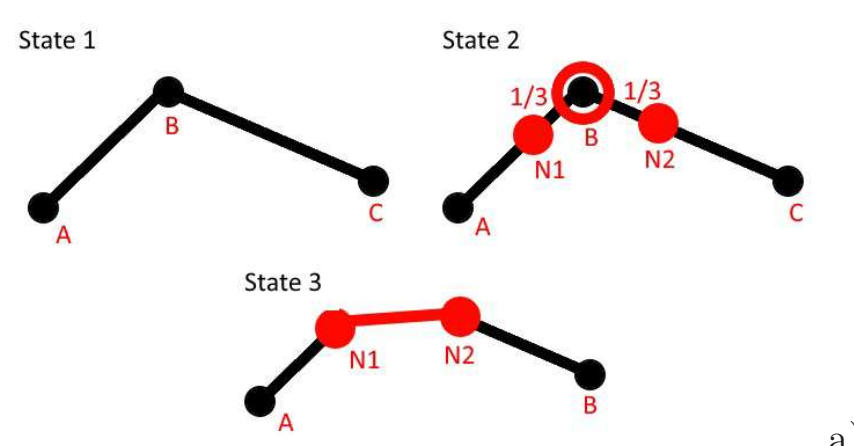

State 1

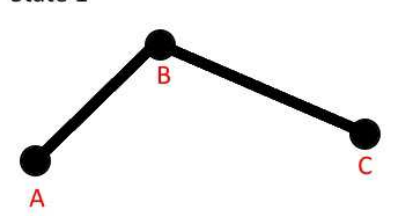

State 3
State 2

a)

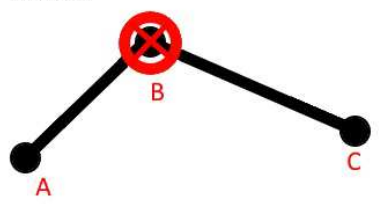

b)

Figure 6.4 - Geometric operations to add or remove wall segments for division's shape adaptation. In a) the process of splitting a point to insert a wall mark in the division polygon is presented. State 1 represents a subset of points belonging to the polygon. $A$ is the previous point, $B$ is the selection point and $C$ is the next point. State 2 represents point selection for segment insertion. A new point $N 1$ is placed at $1 / 3$ of the length between point $B$ and point $A$ and a point $N 2$ is placed at a distance based on the same proportion between point $B$ and point $C$. In Step 3, point $B$ is replaced by the new couple of points. The process occurs in two phases: phase one is the testing phase that consists in replacement simulations to select the point which has associated the most lengthy segment, while phase two performs the substitution of the previously selected point by that associated segment. In b) the process of deleting a point to remove wall marks in the division polygon is depicted. State 1 depicts three points: $A, B$ and $C$. The second state indicates the selection of point $B$. Finally, State 3 shows the deletion of point $B$ and the connection of point $A$ to point $C$, directly. This process is engaged in keeping the division's shape with the greater area as possible.

Although higher limiting values do not seem to represent a problematic issue for the effectiveness of the proposed procedural modelling methodology, the established limit of 8 wall segments foresees to restrain the computational burden. Besides, this value seems reasonable to demonstrate the goal of the process that regards wall adaptation. 
- The minimum number of wall segments is 4 . If the defined number of division walls provided by rules is lower than 4 , it is overwritten to the referred minimum value. The value 4 was defined to ensure that all of the connecting divisions maintain their adjacent wall segments to support transitions, even in the most demanding scenarios (for example, the case of a central division surrounded by other connecting divisions, in which each wall segment belonging to that central division is in contact with a room, at least).

- If the value 0 is provided, the adjustment step for division wall is ignored for the respective division.

Some considerations about the worst case scenario on each operation regarding wall adaptation need to be clarified. Central divisions fully connected with all neighbours and shaped with 4 wall segments constitute the most demanding case when the 8 walls are required by definition rules. In this case, a lack of space compromising transitions placement prevents a well-distributed operation regarding wall segments addition, causing rounded corners. On the other hand, a division placed near at one of the building layout margins, at its center, and requiring the minimum number of wall segments (4 segments) would constitute the worst case for wall segment removal. Due to the restriction of 4 wall segments at minimum, the inner transitions are always ensured. However, requirements regarding entry doors (access to the building exterior) will be ignored whenever divisions in those conditions lose the adjacency with the building layout.

After wall segments adaptation stage, the procedural modelling generation process is resumed with the operations already addressed in previous chapters 4 and 5 . The division graph, already specified by the ontology-based grammar (definition) rules and now loaded into an ontology-based class set is used to place the transition doors between connecting divisions. Afterwards, windows and entry doors are properly placed in the virtual building layout limits.

A last set of operations involving extrusions then takes place to attain the 3D virtual building model. So, the walls are extruded considering the aforementioned transitions. Finally, grounds and ceilings, roofs are produced along with some building 
completions that aim to enhance the 3D model with details.

\subsubsection{Stochastic rules selection for the automatic generation of virtual buildings}

The proposed stochastic process (Figure 6.5) aims to constitute a preliminary and experimental mechanism for the random production of virtual buildings, thus providing a modest but reliable alternative for automating the production of buildings using the proposed procedural modelling methodology. There were proposed two approaches for stochastic generation: one for producing random virtual building with a desired division set and building configuration in which a list of divisions has to be provided along with the required configuration as input; and other to generate a set of random buildings (with random configurations) with aleatory divisions' sets in which the number of buildings to be generated is the only parameter needed. After being provided with the proper minimal parameters, both approaches ensure a fully automatic generation process based on interrelated node graphs.

The selections involved on the former approach are made considering a given divisions' list that gathers priority-one and priority-two divisions (provided as input) and also a building configuration (ranging L-shaped, U-shaped or convex). This approach starts by isolating priority-one and priority-two divisions in two different lists. Then, priority-one divisions are picked up: they can be social (e.g. living rooms) or service-oriented (e.g. kitchens). The selection relies on a pseudo-random generation number approach ranging the list of priority-one divisions. By convention, those divisions are set as entry rooms that provide access to the virtual building's exterior. The first layer graph is automatically composed with the selected priorityone division nodes which are set to be interconnected (a node connection implies a connection between associated divisions which will have a common transition). Moreover, a set of marginal nodes representing the boundaries of those priority-one divisions are created and categorized as top, bottom, left or right. The only exception regards the contacting margins of the priority-one divisions which need to be 


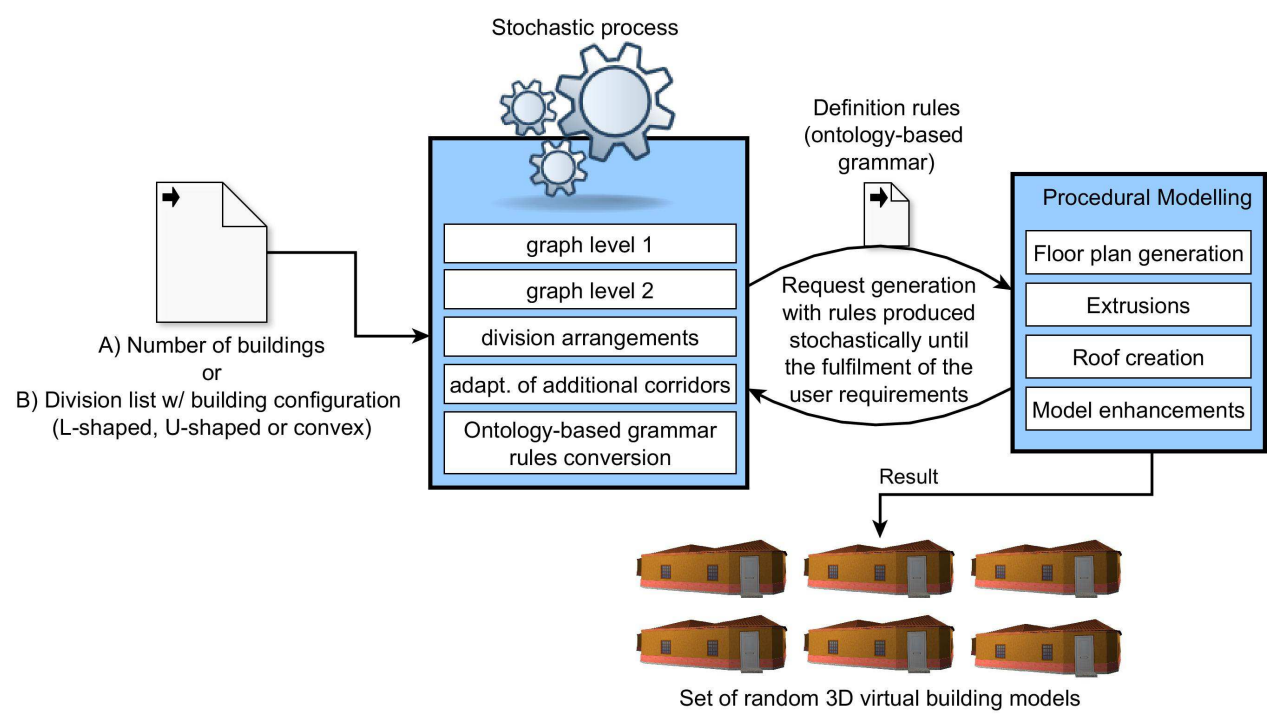

Figure 6.5 - Stochastic process regarding the generation of random virtual buildings. Two stochastic approaches are proposed: one for generating a random building based in an input that specifies a list of divisions and building configuration; and other for producing a specific number of random virtual buildings, in which the referred number is provided as input. In the first approach, the stochastic process mounts two layer graphs, arranges the divisions, adapts additional corridors (if needed) and adds a strategic node representing an empty building part (if a $\mathrm{L}$ or a $\mathrm{U}$-shaped building configuration is required) to produce a structure that is then converted into ontology-based grammar definition rules; the resulting rules are then validated by the moderation process and submitted to the procedural modelling process, which produces the 3D virtual building model. The second approach works iteratively. For each building to be generated, the following steps are performed: the stochastic process starts by selecting, autonomously, a random set of divisions; a building configuration is also randomly picked (L-shaped, U-shaped or convex are the available options); then, the first approach steps are applied - the construction of the graphs along with the arrangement of divisions and adaptation of corridors - to obtain the ontology-based rules that are then validated through the rules moderation process and passed to the procedural modelling process for the proper transformation into 3D virtual model. The approach ends its tasks when the number of generated virtual buildings complies with the input value.

unobstructed to allow the interconnectivity of those divisions. For example, considering two priority-one division nodes with a side-by-side disposition, the right margin of the left node and the left margin of the right node are not created to enable the aforementioned connection between those divisions. Such margins can be seen as temporary nodes - representing temporary containers - for arrangement 
purposes. Furthermore, there is a second step that consists in mounting the second layer graph of division nodes. Towards this goal, priority-two divisions (i.e. private rooms such as bedrooms and toilets) are consecutively picked from their list using a pseudo-random number generator. Each randomly selected priority-two division is converted to a node and associated to a priority-one division margin. A pseudo-random number generator is used once again in two phases, one for attributing a priority-one division node and other for selecting its margin. The second layer graph is considered completed when all the priority-two division nodes have an associated margin belonging to a particular priority-one division node. Then, some nodes representing corridors might be attached between the divisions belonging to both layers to ensure a fully connectable floor plan definition or, in other words, to avoid isolated divisions (for example, a corridor node has to be placed between a marginal bathroom and a priority-one kitchen to avoid the isolation of that bathroom, since those division types are not directly connectable). A simplified scheme depicting the aforementioned main steps is presented in Figure 6.6. Finally, the requirements regarding the building configuration must be satisfied. The following topics will expose the three supported configurations along with the process actions to achieve a valid definition for each case:

- Convex configuration: the process does not make any alteration to the nodebased structure which stays as-is.

- L-shaped configuration: a special node marked as empty is added to one of the extreme nodes representing a priority-one division. Thus, during the nodebased structure conversion into ontology-based rules, that priority-one division node holding the special empty node is unfolded in two building parts rules: one defining a disposable building part at the building corner and other properly loaded with the specifications of the priority-one division node (the carrier).

- U-shaped configuration: to achieve that one, a new priority-one division node has to be created with a corridor node and a special empty node. Then, that node is attached between two consecutive priority-one division nodes while 
their connections are properly adapted to link to the corridor within this new node. By this way, it is achieved the intention of defining a disposable building part in the middle of a building's margin to form the U-shape (or some similar variant), during the conversion of the node-based structure into grammarbased rules.

It is noteworthy that the use of different area levels, specifically room priorities and double-level layer graphs, is based on the suggestions left by Lopes et al. (2010) and Marson and Musse (2010).

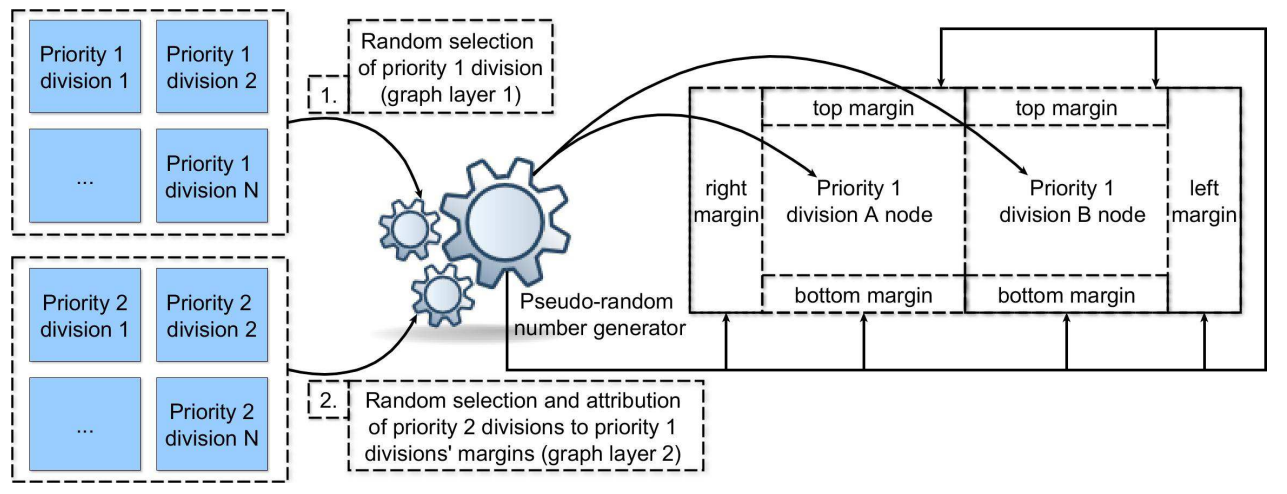

Figure 6.6 - Simplified scheme of the stochastic process, depicting the main steps regarding the random selection and arrangement of both priority-one and priority-two divisions. For a given division list (provided as input or stochastically determined) priority-one and prioritytwo divisions are separated in two distinct sub-lists. Then, priority-one divisions are randomly selected and interconnected and, for each one, nodes representing margins are attached (the contacting sides belonging to different connecting priority-one nodes are not provided with such margin nodes to avoid future conflicts regarding the placement of transitions). Finally, prioritytwo divisions are randomly selected and distributed by the previously created margins belonging to priority-one divisions. Configurations regarding the building format are not addressed in this scheme.

The latter stochastic approach - that only requires the number of virtual buildings to be produced as an input - has an additional starting step that relies on specifying rooms' list and building's configuration, for each building to be generated. Thus, a set of pseudo-random number generations selects one or two priority-one divisions namely a kitchen and or a living room (a division extension created for the stochastic process). Then, priority-two divisions are selected. For each type, specifically private rooms and bathrooms, a random number is generated to determine the number 
of type instances that will integrate the list being created (e.g., if the pseudo-random generator retrieves the number 6 for private rooms then the reference for this room type is added six times to the divisions list under construction). When the division list is completed, a random building configuration is automatically chosen from the supported formats (L-shaped, U-shaped and convex). Afterwards, the previously described stochastic approach is applied to randomly arrange those rooms in a double-layer graph-based structure.

After obtaining the graph-based structure representing divisions' arrangements and connections, these structural definitions are transformed into ontology-based grammar rules for the application of the aforementioned process moderation process, followed by the procedural modelling process responsible for creating the virtual building model. This is valid for both stochastic approaches.

It should be emphasised that the defined stochastic process is not concerned with real-world architectural rules. It only constitutes a process for the random virtual building generation that aims to overcome the deterministic nature of the treemap approach adopted for this proposed procedural modelling methodology.

\subsection{Final Procedural Modelling Methodology Im- plementation}

A software system integrating two tools - deterministic and stochastic - was developed to support virtual buildings generation using the final procedural modelling methodology proposal. The deterministic tool relies on a graphical user interface (GUI) that allows to draw the desired floor plans and to parametrize each operation. Regarding the stochastic tool, it produces random virtual buildings, considering high-level requirements. Both tools are depicted in Figure 6.7.

Besides the aforementioned tools, the software toolkit also integrates a backoffice that enables the user to adjust default parameters. Such parameters map the restriction rules identified on chapter 5 and are used by the procedural modelling 


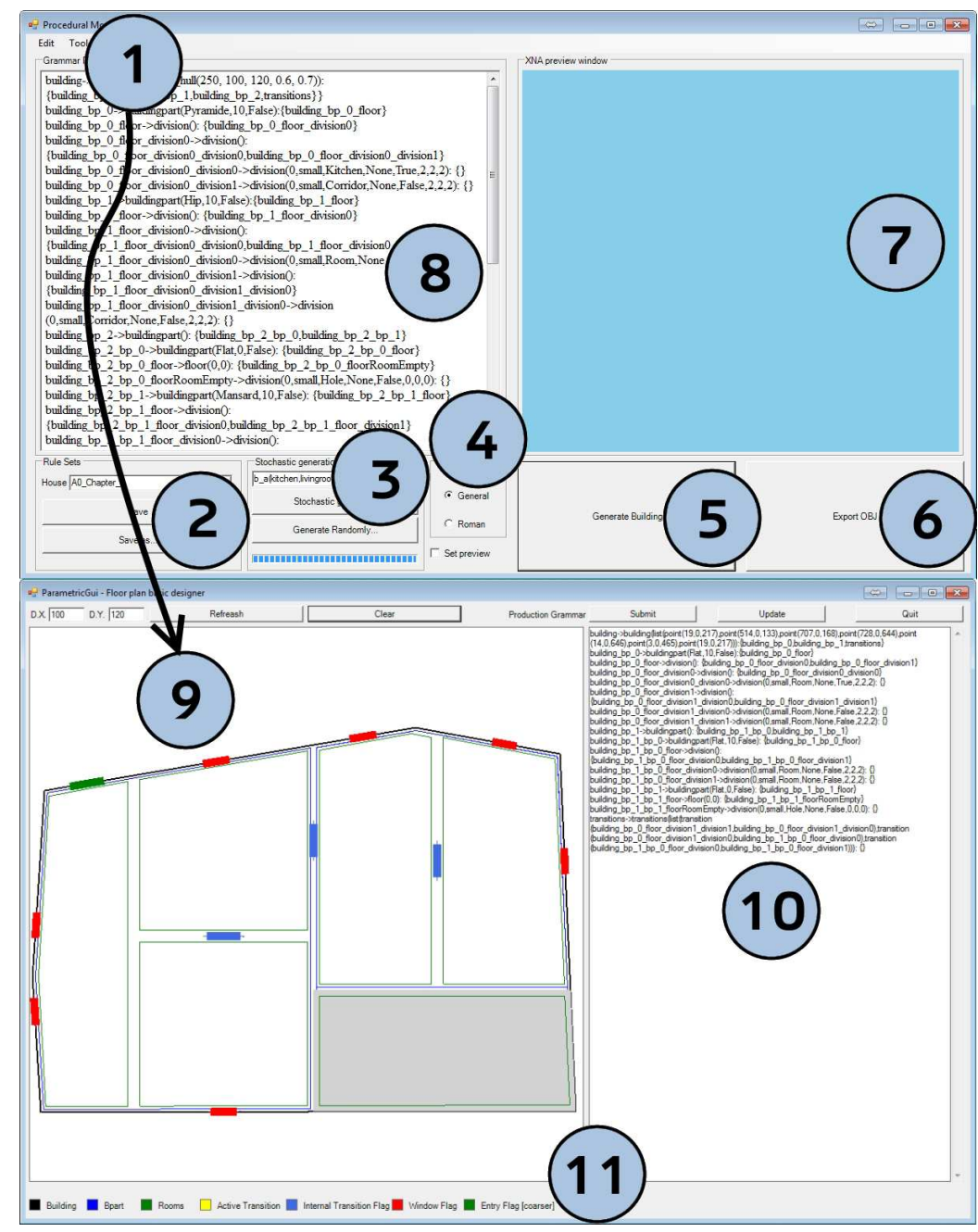

Figure 6.7 - Screenshot of the procedural modelling system implementing the stochastic and parametric tools. The upper part of the figure depicts the main GUI, which is composed of the following: (1) access to the deterministic parametric tool; (2) a file area to manage the stored grammatical rules; (3) a stochastic menu area that allows the user to rapidly generate a building with a specific list of divisions or even a set of random buildings; (4) the style, which can be general or roman; (5) a button to request the generation of a building regarding the grammatical rules contained in (8); (6) a button to export the building in wavefront obj format; (7) the XNA floor plan previewer; and (8) a grammatical rules area. The lower part of the image presents the deterministic parametric tool: (9) the floor plan drawing area with several colours to help distinguish the input elements; (10) an area that presents the grammatical rules during user drawings and parametrization; and (11) captions for the colours used to identify the drawn elements. 
implementation in strategic steps of a building generation. Thus, one can modify the textures of the different elements (e.g., doors, walls, windows, roof) and the values associated with the construction of the models and geometrical operations (e.g., the definition of door occupation in a wall segment, weight attribution for the reserved grammatical words "big", "medium" and "small" wall thickness). These possibilities allow the customization of the final models and partial control of the modeller's behaviour. Figure 6.8 presents two excerpts of the implemented backoffice interface, one exposing a texture sub-package and the other one showing a text box-based layout for the parametrization of values that are considered during the generation process.
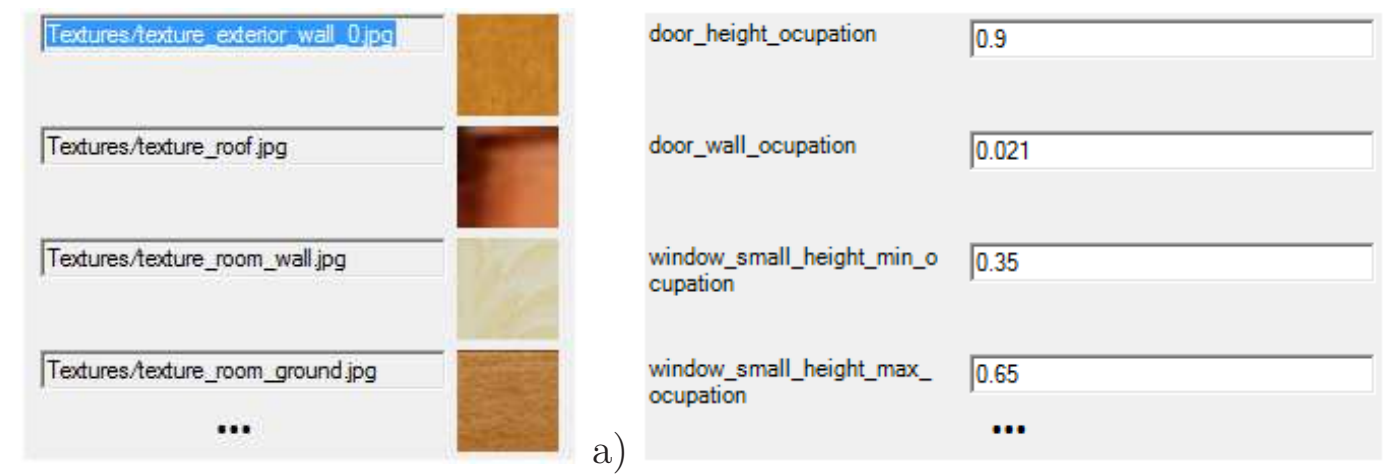

b)

Figure 6.8 - Software toolkit backoffice screenshots excerpts regarding texture and restriction parameters: a) depicts a sub-package that allows the modification of each element's texture; b) is a text-box-based layout for the parametrization of values that are considered by the modeller during the generation process.

The stochastic and deterministic tools that make part of this toolkit will be detailed in the next subsections.

\subsubsection{Deterministic Tool}

To rapidly create virtual building models while ensuring a certain level of step-bystep control by the user, a parametric tool was developed (Figure 6.7, bottom). This tool is capable of generating grammatical rules through a small number of user interactions. The user starts by defining the building's shape (its outline contour). Then, a set of building parts must be specified. These building parts can also be subdivided 
into other building parts for arrangement purposes. If a building part is divided in the horizontal orientation, then subsequent containers inside of it will produce vertically arranged building parts. According to the defined grammar, this parametric tool requests the roof type and the building part height, through a graphical prompt window opportunely presented to allow the parametrization of a building parts. A height defined with a value of 0 specifies a disposable part (such building parts are removed right after the floor plan division stage foreseeing the creation of building layouts with holes and/or non-convex configurations). Then, divisions can be integrated inside the building parts. Through mouse clicking, the user can populate a building part with divisions (rooms). Once again, division arrangement is allowed through the definition of final divisions inside division containers. The division direction behaves similarly to that which was explained for building parts: divisions are arranged vertically if the container has a horizontal orientation, and vice versa. The parametric tool asks for the set of parameters that should define a given division, such as the number of delimiting walls, division type, number of windows, internal structure and flag for entry doors (transitions interfacing the building exterior), using a prompt window as it occurs in building parts parametrization. The tool also supports the easy definition of division connectivity. The simple act of right clicking upon the starting division and selecting the target division for connection is sufficient to generate the rule that relates them in terms of transitions.

It is also important to stress out that the tool asks for parameters according to the operation's context. For example, if a division is being defined, then only the required division parameters are asked to comply with the proper grammatical rule. This is helpful in reducing malformed grammatical rules, which could result in incoherent models. Figure 6.9 depicts the tasks that can be performed by user, using this parametric tool to produce a virtual building, deterministically.

\subsubsection{Stochastic tool}

The manageable and flexible nature of the treemap approach allowed the development of a semi-/fully automatic tool (Figure 6.7, top) that implements a process 


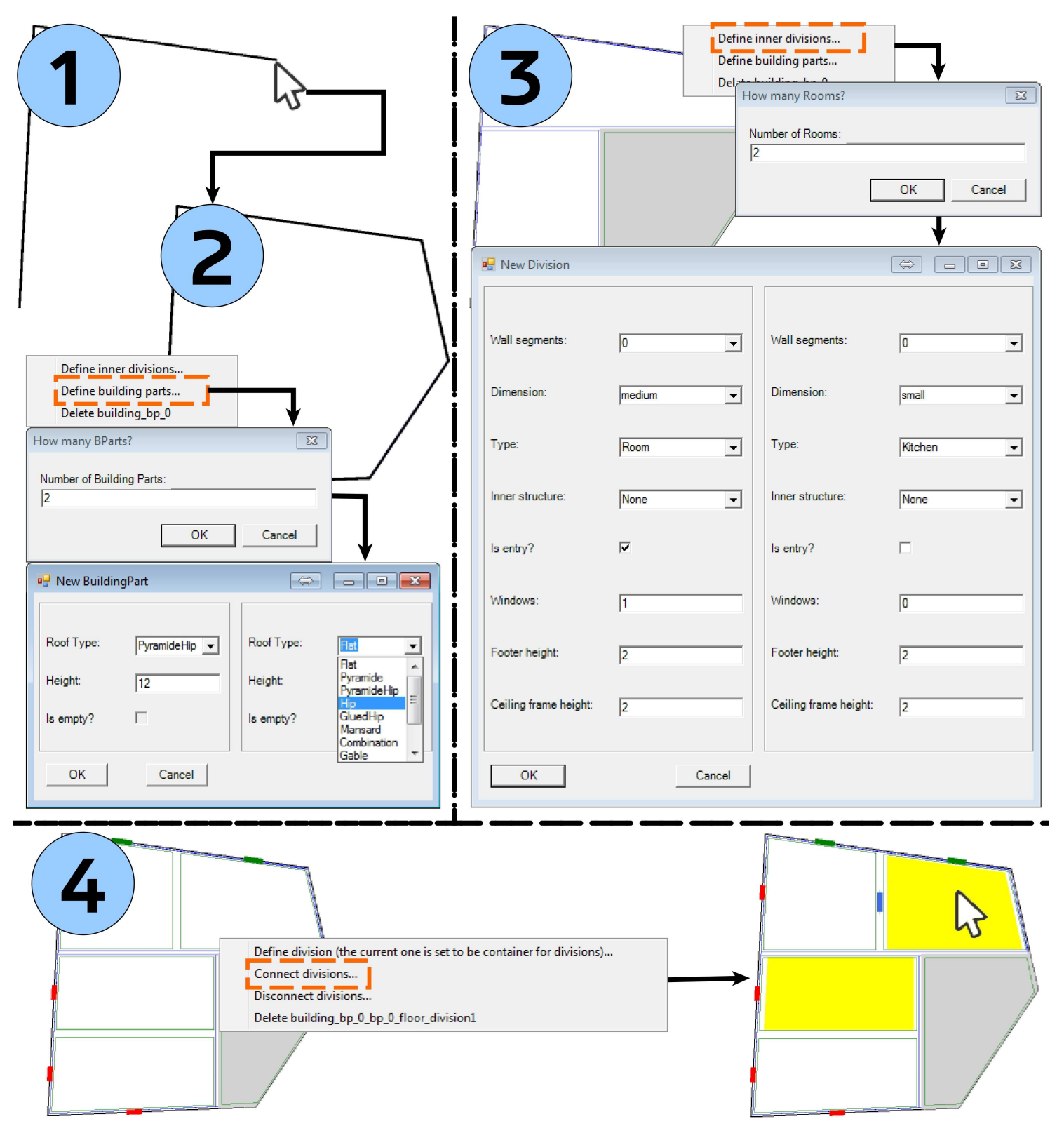

Figure 6.9 - Set of operations that a user can perform in the deterministic tool's GUI which consists in a simplistic viewport that enables the definition of a building. This definition is made through the constraint polygon drawing (1), specification of building parts (2) - defined inside building's polygon or inside other building parts - and divisions (3) - defined inside building parts or other divisions - and also context-aware parametrizations. The splitting into building parts and divisions occurs automatically, sparing the user of tedious manual drawings. Moreover, each connection can be specified by selecting the starting and targeting divisions involved, while a yellow highlight provides the proper feedback (4). 
capable of producing random virtual buildings.

As input, the tool accepts a divisions list which has to be selected by the user through a combo-box control (Figure 6.7, circle 3) that contains a preloaded set of division lists in the following format:

building_name( room0, room1, room2, ..., roomN) [configuration]

The selected divisions are then divided into a couple of lists, accordingly with their priorities. Afterwards, priority-one divisions (kitchens or living rooms) are selected and connected and some marginal areas are also attached to each division (avoiding interference with the referred connection). Then, priority-two divisions are related to priority-one divisions' margins using pseudo-random attributions and some corridors are created to ensure connectivity between those two categories of rooms. The process results in a hierarchical node-based structure. The last field - configuration - refers to the building's configuration and intends to provide the possibility of producing L-shaped, U-shaped or fully convex buildings, on demand. L-shaped or U-shaped configurations require the integration of a node representing a disposable building part. Such node is strategically attached to the previously determined nodebased structure to properly define an empty building part for one of the building's corners (L-shape case) or at the center of one of its margins (U-shape case).

Alternatively, the user can produce a set of virtual buildings based on a unique input: the desired number of buildings to be produced (Figure 6.7, circle 3, second button). After that, an iterative and automatic process to produce the required number of buildings is started, and two main tasks are executed for each building. The first one is responsible for the pseudo-random selection of a divisions list, for each building to be generated. The following conditions affect each list selection: there can be one or two priority-one divisions; the number of private divisions cannot exceed nine; the number of bathrooms cannot overcome the number of private rooms. Next, a building configuration is also randomly picked from the following available set: Lshape, U-shape or convex. Afterwards, the second task takes place accordingly to which was already explained in the previous paragraph. The process executes until the number of generated rooms complies the user requirements. 
The outputs of these tools are ontology-based grammar rules that are forced to pass through a pair of built-in modules aiming the generation of the final virtual building model (similarly to the system presented on chapter 5): the rules moderation module and procedural generation module. The first module validates the grammar-based definition rules, loads an ontology-based class set and ensures the feasibility of the floor plan through a probing process. Then, the class set is submitted to the procedural generation module which applies a set of steps - included in the main stages responsible for the effective floor plan production, building extrusions and also building completions - to achieve the final form of the 3D building model.

\subsection{Preliminary Tests and Results}

A set of structures was produced using the presented deterministic tool that implements the latest procedural modelling methodology features. Figure 6.10 provides a first insight about the capabilities of the methodology's last version in generating different kinds of traversable buildings, in particular one constrained by a pure convex shape, another one exposing the results of the fake-concave strategy application and a last building depicting the wall's adaptation results regarding interior divisions.

An experience was also carried out to demonstrate the applicability of the stochastic approach, through its homologous tool. Thus, a set of L-shaped buildings was produced considering the same set of divisions: a kitchen, a living room and three rooms. As it is depicted in Figure 6.11, for each generation the referred set of divisions is maintained but their organization is randomly altered. Corridors are also created, in some cases, to ensure the connectivity between all inner divisions. 


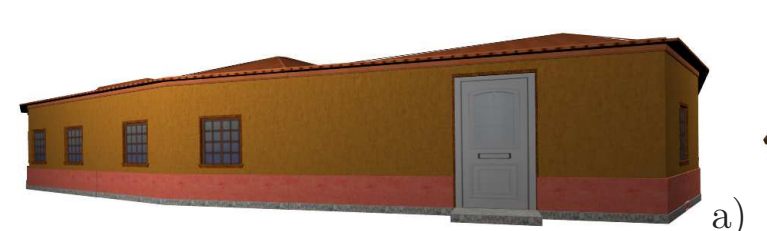

a)
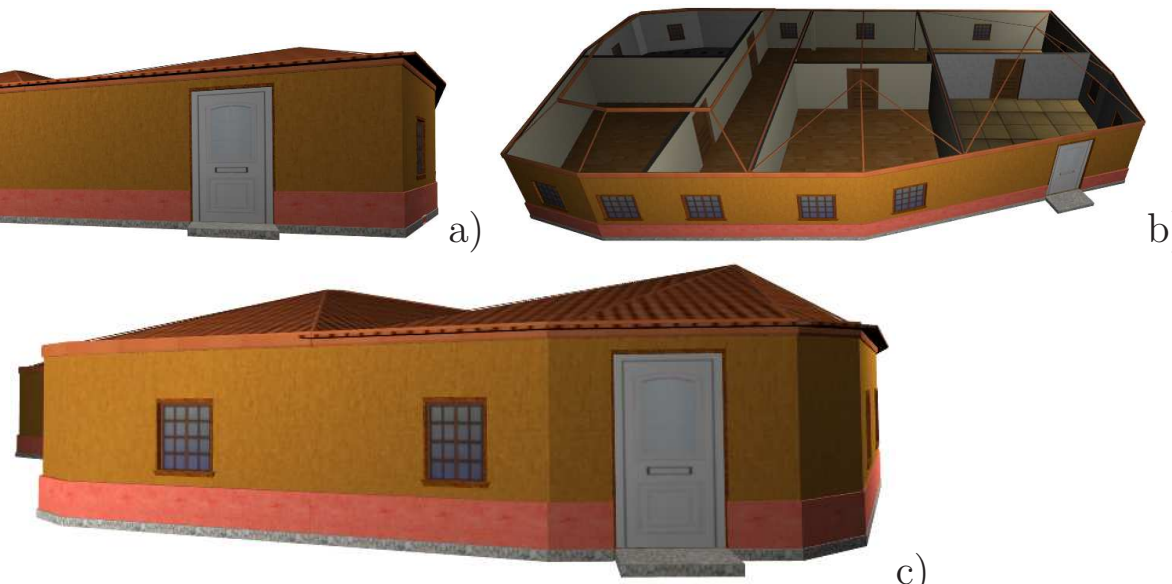

b)

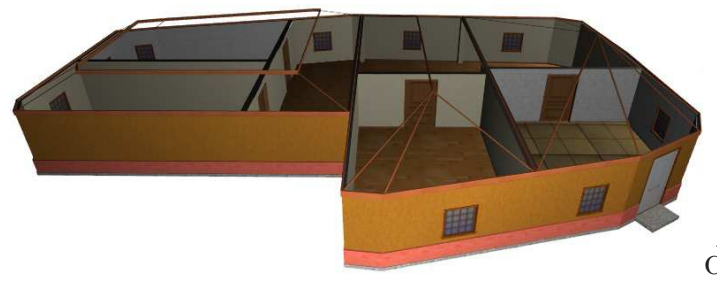

c)

Figure 6.10 - Preliminary results depicting the traversable buildings generated with the final methodology: a) and b) show a traversable building composed by three connected corridors, a kitchen, a bathroom and two bedrooms; they were generated regarding a pure convex constraint polygon; c) d) and e) have a similar configuration and are constrained by non-convex polygons due to the fake-concave technique; e) also presents the results of a inner wall definition for the geometric adaptation of inner divisions.

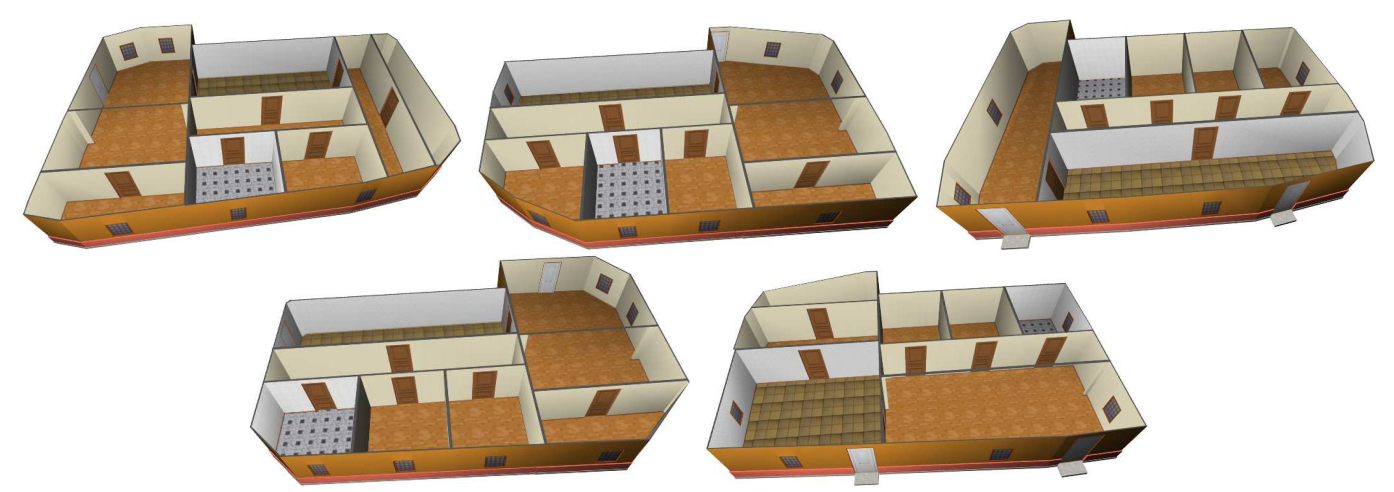

Figure 6.11 - Preliminary results of the stochastic approach: five generations were made using the same set of rooms (divisions) - a kitchen a living room and three rooms. The result is a set of L-shape buildings that hold the same set of divisions but with random arrangements. 


\subsection{Summary}

This chapter presented a semi-automatic methodology capable of producing 3D buildings constrained by arbitrary shapes in which the generation process is guided by sets of grammar rules, that resulted from the definition of an abstract ontology for describing buildings generically. This version of the methodology supports the definition of building constraints which can be geometrically convex or not, due to fake-concave technique. Moreover, the wall segments of the interior divisions can also be altered accordingly with the grammar rules to modify divisions' geometry. A stochastic process was also engaged with the final procedural modelling methodology to enable the automatic generation of random buildings. In short, a set of divisions or a desired number of structures to be generated are provided as input to trigger a randomized generation of virtual buildings.

Also, a system implementation regarding the proposed final methodology was presented with two tools: a deterministic tool that enables hand-made floor plan drawings and parametrizations and a stochastic tool capable of producing random buildings with low user interaction. The former makes a progressive definition of ontologybased grammar rules while the user is drawing and parametrizing the desired floor plan. The latter autonomously produces a double-layer division graph that is converted into grammar rules. Two types of inputs in the form of simple parameters are sufficient to trigger the action of this second tool: a list of divisions or a number of virtual buildings to be generated.

To accomplish the virtual building generation task, the grammar-based rules (also known as definition rules) produced by both of the aforementioned tools are verified by the rules moderation module and, after proper validation, they are forwarded to the procedural modelling module which is responsible for producing the $3 \mathrm{D}$ virtual building enhanced with some details like inner footers and stair-steps for entry doors.

The preliminary tests provide a first insight about the capabilities of both methodology and stochastic process. However, an extended set of results will be presented 
in the next chapter which is reserved for the evaluation of the final procedural modelling methodology version. 



\section{7 \\ Procedural Modelling Methodology Evaluation}

In this chapter, results from the application of the procedural modelling methodology exposed in chapter 6 , namely through the developed toolkit, will be presented. This toolkit consists in a basic graphical interface composed by two tools. One of these tools aims the deterministic generation of buildings considering the user floor plan drawings and proper parametrization. The other tool - the stochastic one - offers the possibility of generating sets of completely random buildings or, on the other hand, a single building with a given configuration and a provided list of divisions to be randomly arranged inside it. Both of these tools produce ontology-based grammar rules (definition rules) and use the two main implemented modules of the toolkit moderation rules and procedural generation - to convert those rules into 3D virtual buildings.

A set of testing virtual building models, generated with the previously referred tool, are presented to demonstrate the methodology capabilities. Firstly, some simple houses constrained by convex polygons will be exposed. Another results subset will show the manipulation of wall segments for inner divisions. Then, the ability and flexibility of the subdivision approach adopted along this work - the treemap - will be demonstrated. Afterwards, a set of buildings randomly generated by the stochastic 
tool will be shown. Moreover, an example of an ontology extension will be presented through the comparison of two generated buildings: a generic house using the base ontology and a roman house using an extension to support architectural elements belonging to the roman style. This example intends to clarify the importance of the building ontology for the methodology support and its flexibility. The results of the fake-concave technique application will be also presented along with the addressed tests. The chapter ends with performance tests to measure both the central processing unit (CPU) and random access memory (RAM) usage, but also the time consumption in the process of generating individual structures composed by a distinct numbers of vertices.

\subsection{Virtual Buildings Constrained by Arbitrary Shapes}

Some tests were performed to validate the proposed methodology, while generating simple buildings based on the generic ontology. According to the grammar rules requirements, the buildings were defined to be constrained by a convex hull. Each building has different configurations, with different division arrangements, connections and structures. Thus, the generated virtual buildings intend to demonstrate the variety and heterogeneity of structures that can be produced by the final version of the procedural modelling methodology.

The first two virtual buildings are presented along with the respective grammar rules to exemplify their usage in the following scenarios: 3D model generation constrained by a pure convex polygon and 3D model generation transformed with the fakeconcave technique. The rest of the results will be presented without the related grammar rules to spare the heavy reading. All of the generated buildings that are going to be presented were produced respecting the limits of a convex polygon outlined randomly by a convex hull generator method. Subsection 7.1.1 presents a simple virtual building, containing only a hall - identified as a common division but with the particularity of connecting all others - a common room, a kitchen and a bathroom. The proposed methodology is capable of producing other types of virtual 
buildings described by non-convex shapes, as it is demonstrated in subsections 7.1.2 and 7.1.3. Subsection 7.1.2 presents an U-shaped house containing a kitchen, a living room, a room and a bathroom. The "U" is formed by an empty space that results from a building part configured to be geometrically disregarded (disposable building part), i.e., when the grammar rule parameter of a given building part height is set to zero (see ontology-based grammar rules in chapter 6), the procedural modelling module treats it as a geometric hole, discarding it after the floor plan definition step. It also works when the height parameter is set to zero. The next building, presented in subsection 7.1.3, describes the generation of an L-shaped house, constituted by a kitchen, a living room, a corridor, two generic rooms and a bathroom. The L-shape was defined by the aforementioned geometric hole method. Finally, in subsection 7.1.4, the generation of a general purpose building with three corridors interconnected by a doorless transition and other six divisions is presented.

\subsubsection{Simple building model}

The simplest building is constrained by a convex polygon and composed of a room, a kitchen, a toilet and a central corridor connecting all of the other divisions. The following grammar-based rules create the virtual building depicted in Figure 7.1.

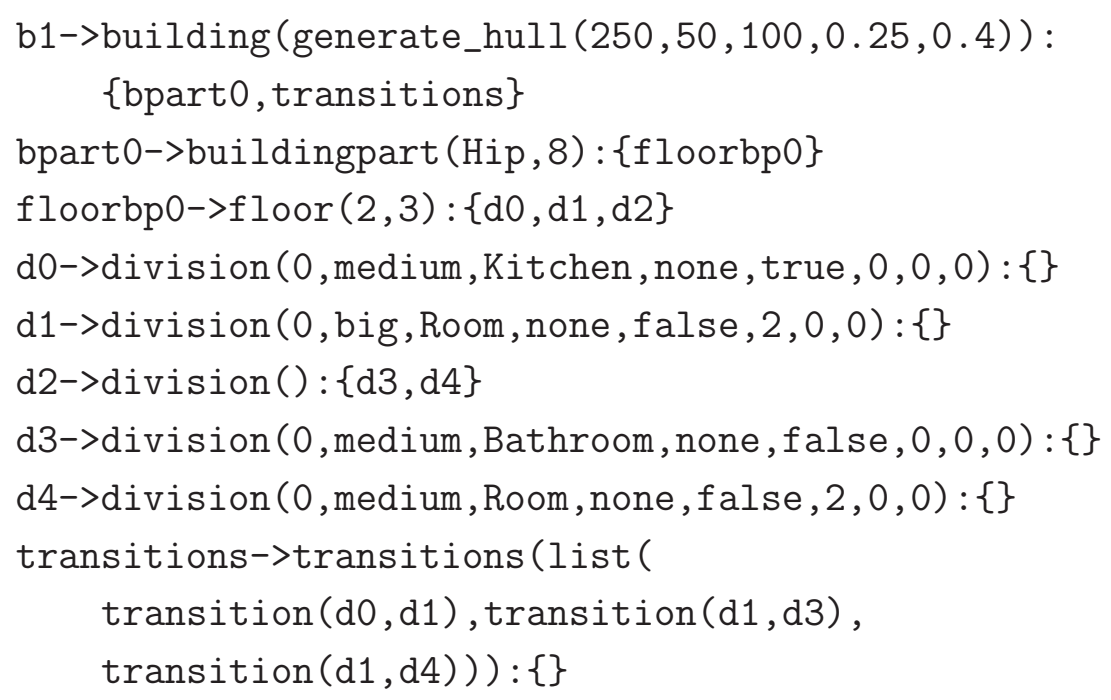




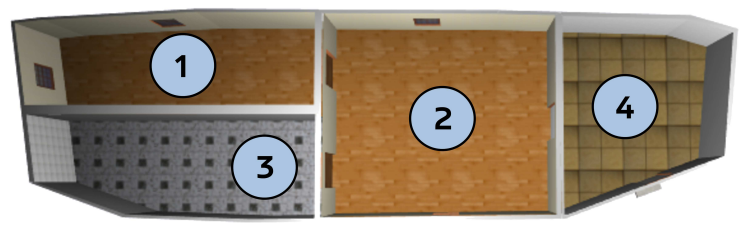

Figure 7.1 - Simple general purpose building (based on the generic ontology) with a room (1), a central hall passage (2), a bathroom (3) and a kitchen (4).

\subsubsection{U-shaped building model}

An U-shaped building takes advantage of the already referred fake-concave technique. The virtual building consists of a kitchen, that provides access to the building and connects to a living room (big division), that in turn interfaces with a room and a bathroom. The next grammar-based rules exemplify the generation of this type of buildings and the result is depicted in Figure 7.2.

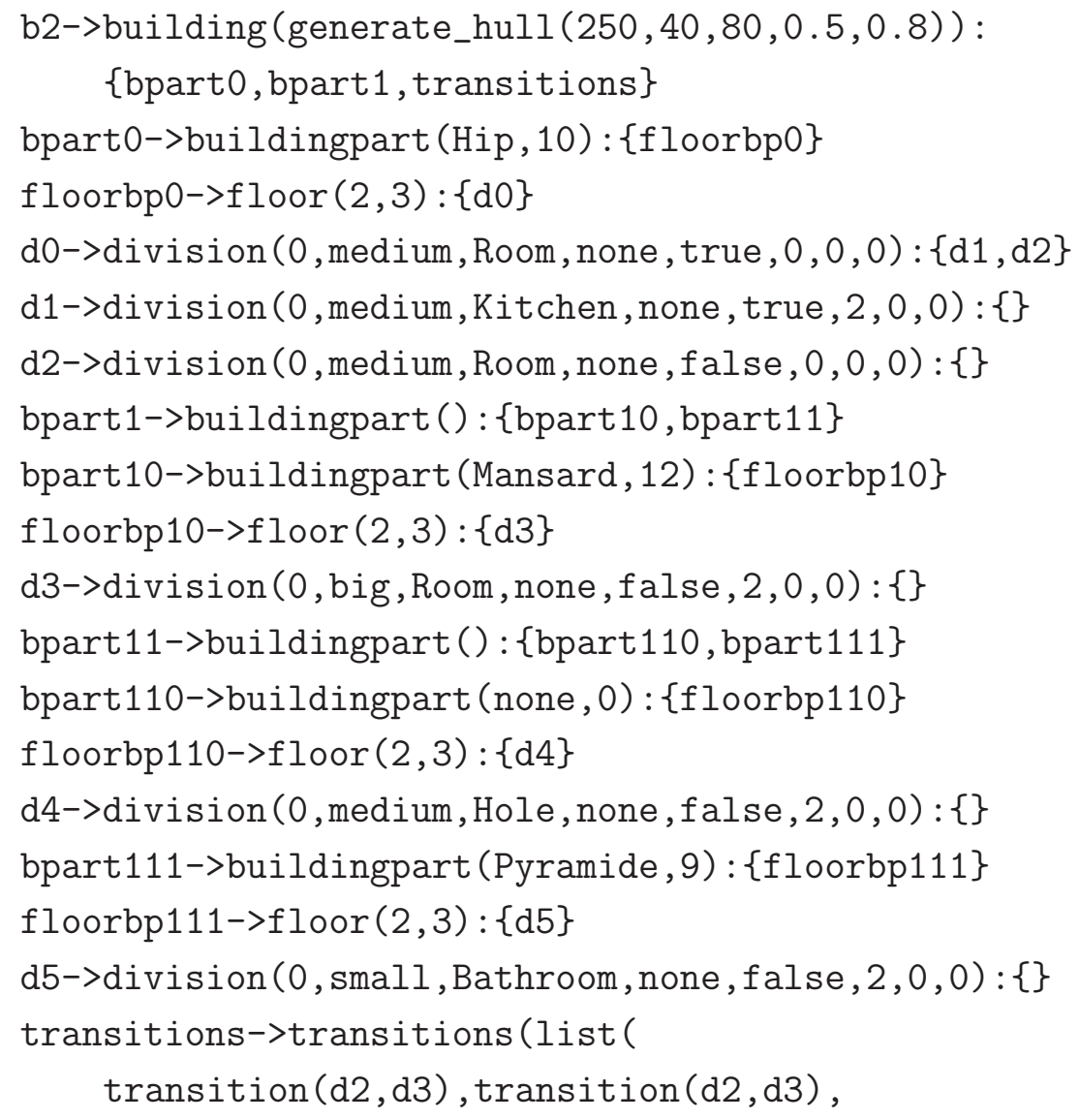


transition $(d 3, d 5))):\{\}$

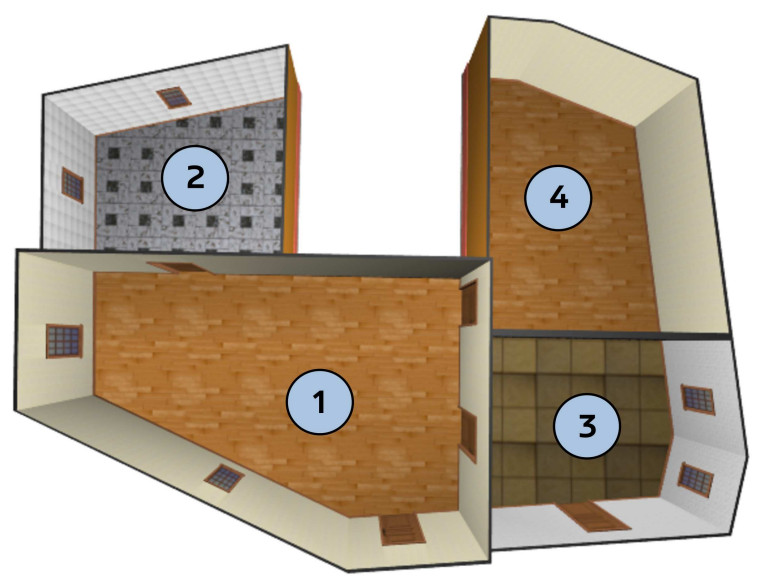

Figure 7.2 - U-shaped general purpose building (based on the generic ontology), with an entry room (1) that connects with a bathroom (2), a kitchen (3) and a private room(4).

\subsubsection{L-shaped building model}

An L-shaped building also takes advantage of the already referred fake-concave strategy. The building consists of a kitchen, two side rooms and two connected central divisions ensuring that all other divisions are reachable. Figure 7.3 depicts the modelling result.

\subsubsection{Arbitrary building model with three connected corri- dors}

This building is constrained by a random convex polygon that contains several rooms, a toilet and also an entry kitchen (Figure 7.4). A central corridor connects to a couple of rooms, a bathroom and also interfaces with two corridors. One of these corridors provides access to the house and the other one connects to an entry kitchen, a smaller bathroom and also to a small room. These corridors are not connected by transition doors. Instead, the procedural modelling system creates a doorless transition to connect them. 


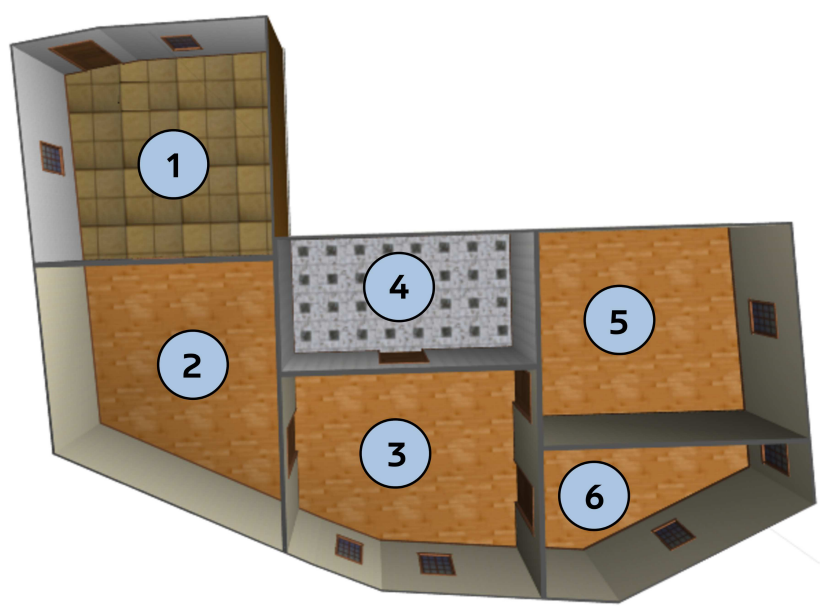

Figure 7.3 - L-shaped general purpose building (based on the generic ontology) with an entry kitchen (1), two connected rooms acting as hall passages (2,3), one of them interfacing with toilet (4) and two private rooms $(5,6)$.)

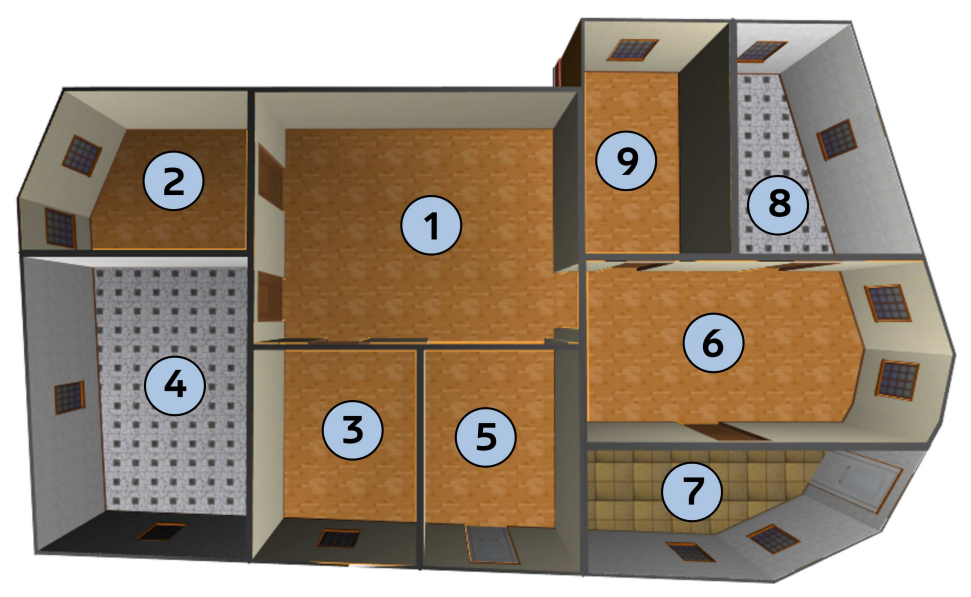

Figure 7.4 - A general purpose building (based on the generic ontology) with three corridors connected through doorless transitions: the central corridor (1) connects to a couple of private rooms (2,3), a bathroom (4), an entry corridor(5) and lateral hallway (6) that, in turn, interfaces with a kitchen (7), another private room (9) and a small bathroom (8).

The set of tests was posteriorly extended to demonstrate the effectiveness of the division wall adaptation process. 


\subsection{Interior Divisions' Walls Adaptation}

In accordance with this work objectives, a process for inner wall adaptation was proposed and described in chapter 6 . This section is focused in presenting the variety of structures that this methodology is capable of generating using the referred method and also considering the holes provided by the fake-concave technique. The parametric tool (described in 6) was used to generate buildings, that will be described in the following subsections. The resulting grammar contains the definitions of the required number of wall segments, for each division. A value equals to 0 means that the wall segments of a given division will remain without any transformation. A value between 0 and 4 is set to the minimum value of wall segments currently supported by the process: 4 . Moreover, a value greater than 8 is changed to the maximum value of wall segments currently supported by the process: 8 . A value between 4 and 8 will trigger the adaptation process to insert or remove wall segments.

\subsubsection{L-shaped building model (six divisions)}

The building model depicted in Figure 7.5 has an entry kitchen formed by 7 inner wall segments. The kitchen interfaces with a flattened long corridor, composed by 6 inner wall segments, that connects to a central hall also with 6 wall segments. This corridor also connects to another corridor composed by 5 wall segments, that, in turn, connects to a couple of divisions: a bathroom with 6 wall segments and a room with 5 wall segments.

\subsubsection{L-shaped building model (nine divisions)}

This building model has an entry kitchen with no adaptation required (0 wall segments), that connects to a side corridor, also with 0 wall segments signalling that no adaptation is required. The corridor connects to a bathroom with 6 wall segments 


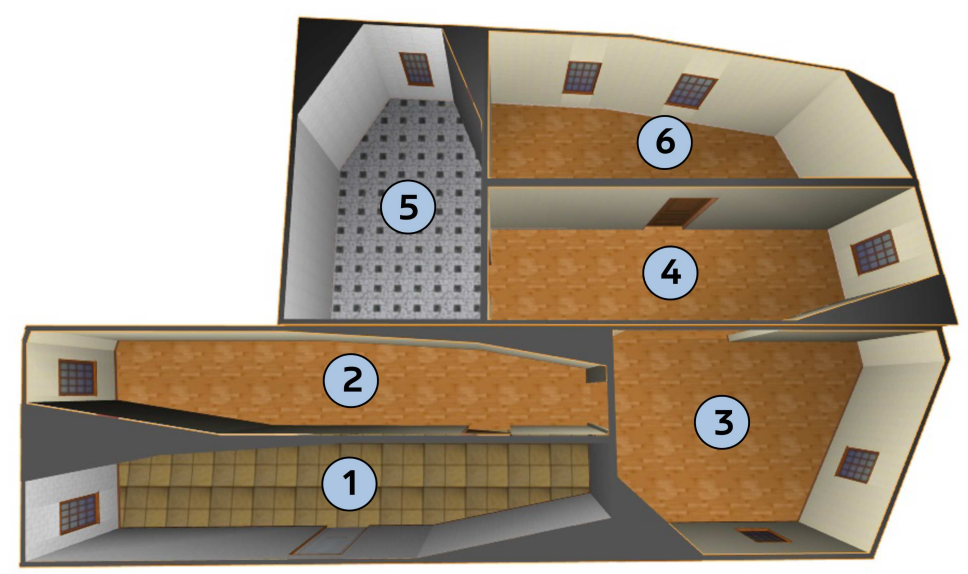

Figure 7.5 - L-shaped building demonstrating inner walls adaptation: it has a set of connected divisions including a kitchen with 7 wall segments (1), two corridors composed by a 6 -segments wall $(2,3)$, a third corridor composed by a 5 -segments wall (4), a bathroom (5) and a private room (6) with 6 and 5 wall segments, respectively.

and to a room with 8 wall segments. It also interfaces with a big corridor containing 6 wall segments that allows traversing to a small entry corridor and to a room with 7 and 6 wall segments, respectively. Moreover, it provides access to another room with 5 wall segments and to a bathroom containing 6 wall segments. Figure 7.6, depicts this building model.

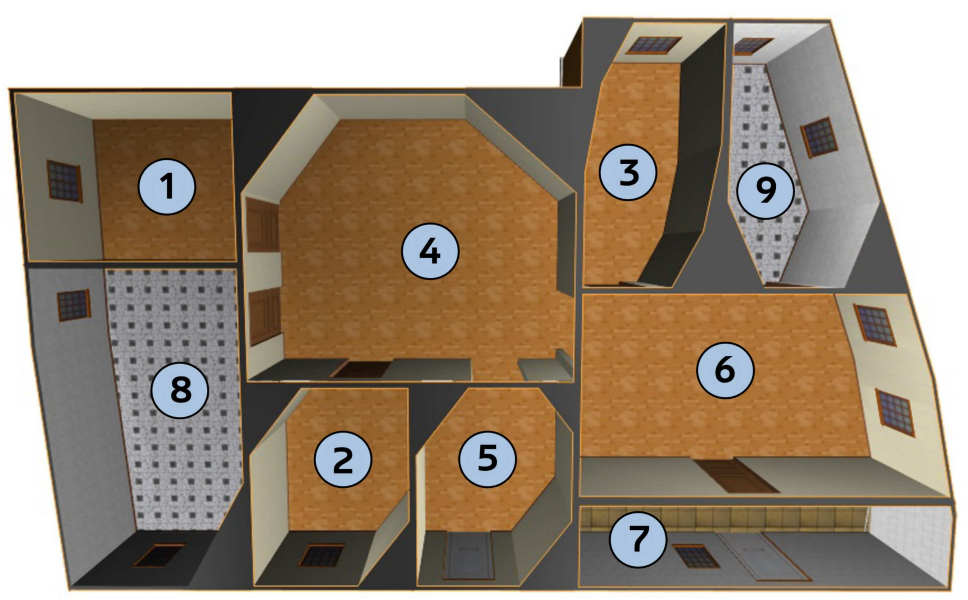

Figure 7.6 - Second L-shaped building that demonstrates inner walls adaptation: it is composed by a set of three rooms $(1,2,3)$ delimited by 5,6 and 8 wall segments, three corridors $(4,5,6)$ with 6,7 and 0 wall segments $(0$ is the value that defines that the division's wall segments remain unchanged), a kitchen ( 7 ) without wall adaptation ( 0 wall segments) and two bathrooms $(8,9)$, both with 6 wall segments. 


\subsubsection{T-shaped building model}

Another building (Figure 7.7), identified as the third house, was generated regarding interior walls that form constraint n-gons to delimit inner divisions. This house is composed by an entry kitchen, forced to contain four wall segments, that connects to a corridor with 7 wall segments which in turn gives access to a couple of divisions: a generic room with 6 wall segments and a bathroom surrounded by 5 wall segments. From it, there is also access to an entry room ( 8 wall segments) that interfaces with a couple of rooms, both with 7 wall segments and to a bathroom with no wall restrictions.

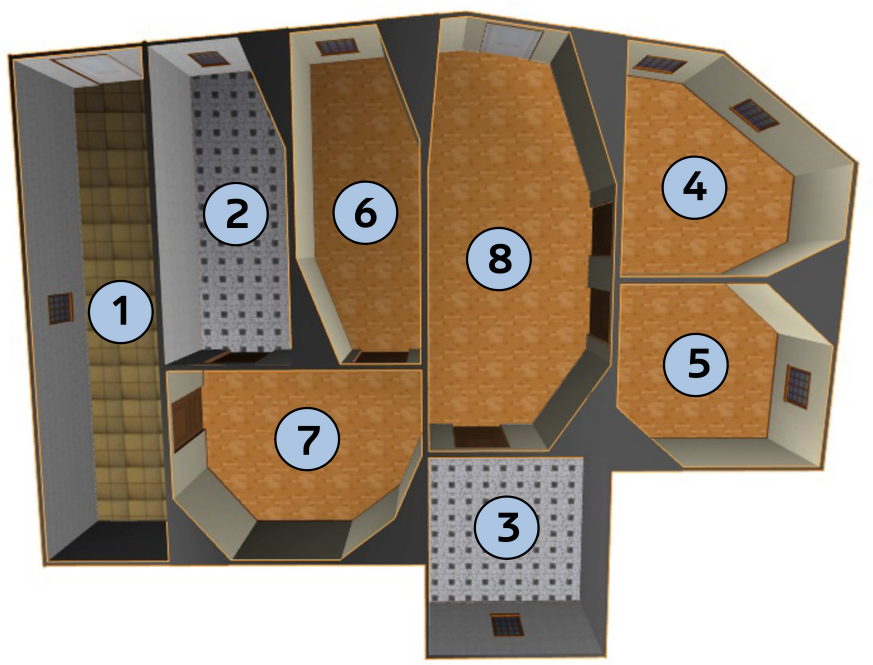

Figure 7.7 - T-shaped building to demonstrate inner wall adaptation. It has a kitchen (1) with 4 walls, two bathrooms $(2,3)$, one having 5 wall segments and other without segment restriction, three private rooms $(4,5,6)$ each one containing 6 wall segments and a couple of connected corridors $(7,8)$ surrounded by 7 and 8 wall segments, respectively.

\subsubsection{Building model with a complex layout}

The last virtual building generated for this section is pretty unconventional, nevertheless, it intends to show the flexibility of the proposed methodology in generating building composed by arbitrary shapes (Figure 7.8). It has an entry kitchen with 5 inner wall segments, that connects to a long corridor with 6 wall segments. This 
corridor, connects to a couple of rooms composed by 7 wall segments each. There is also a transition to another corridor constrained by 8 wall segments that interfaces with a bathroom and with yet another corridor, each one formed by 6 wall segments (almost imperceptible due to the similar orientations of the consecutive wall segments). Finally, this last corridor provides access to a room with 5 wall segments.

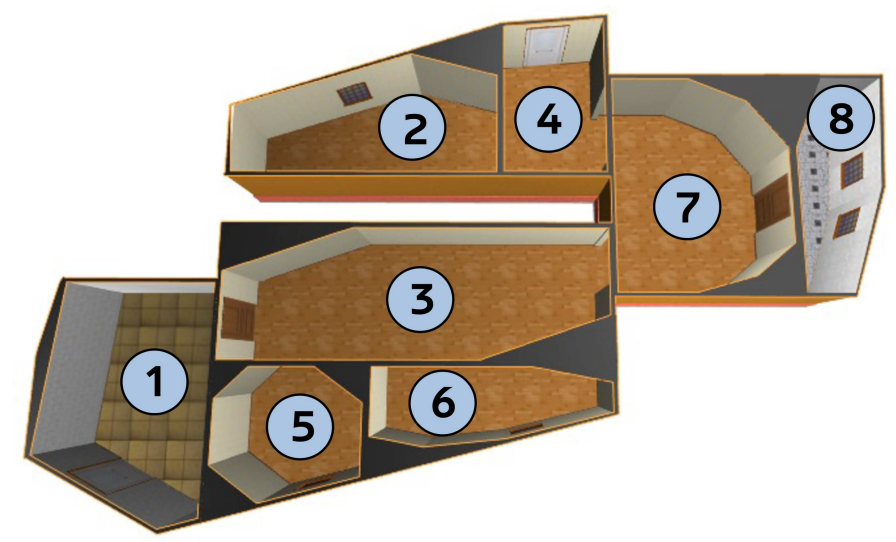

Figure 7.8 - Fourth virtual building model for the demonstration of inner walls adaptation: it is composed by a kitchen (1) and a room (2) with 5 wall segments each, a couple of corridors $(3,4)$ both constrained by 6 wall segments, a couple of rooms $(5,6)$ with 7 wall segments each, yet another corridor (7) having 8 wall segments and a bathroom with 6 wall segments (8).

\subsection{Subdivision Provided by the Treemap Approach}

The adopted treemap approach was also tested to demonstrate floor plan's division process effectiveness and flexibility. The objective is to show is to demonstrate that treemap is capable of producing more than nine divisions, outperforming the tests made in the last section. Thus, using grammar rules, four different structures were created with distinct morphologies and also division sets, all of them connected to each other to ensure that all divisions are reachable. The first model is constrained by a pure convex shape and contains only ten divisions. The second structure is a bit more complex and has fifteen divisions and two holes formed by disposable building parts. The third virtual model is composed by three holes using fakeconcave approach. It has twenty reachable inner divisions. The most complex 
model includes thirty divisions and four disposable building parts (based on the fake-concave technique), constituting the holes of the structure. Figure 7.9 depicts these four models.

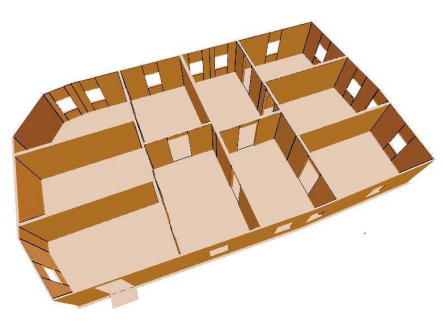

a)

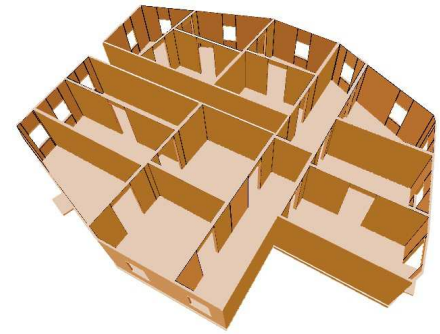

b)

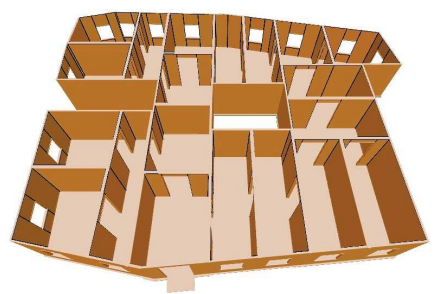

c)

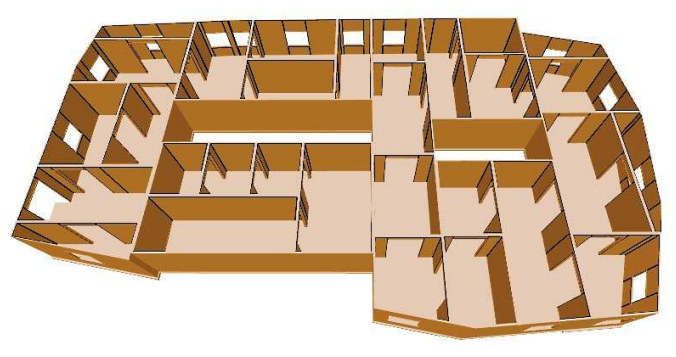

d)

Figure 7.9 - Floor plan subdivision examples with four distinct structures: a) exposes a structure with 10 divisions in a pure convex polygon. b), c) and d) depict fake-concave structures with 15, 20 and 30 divisions, respectively.

\subsection{Generating Structures Stochastically}

Tests made using the stochastic process for the automatic generation of virtual buildings, described in chapter 6, are presented in this section. Four groups of tests were made. The first set focused the generation of buildings constrained by a convex shape without holes, while the second and the third sets demonstrate that the proposed stochastic process can also be employed for the production of $\mathrm{L}$ and $\mathrm{U}$ shaped buildings, respectively. Moreover, the models of the first three groups of tests were generated considering two priority-one division nodes - a kitchen and a living room, that can be considered perfectly usual service/public divisions - and a fixed set of priority-two division nodes, that were randomly distributed 
by the margins of the referred priority-one division nodes: three private rooms and one bathroom. The last set of virtual buildings enabled the fully automatic production of $3 \mathrm{D}$ buildings in a few minutes and with only a few inputs (a mouse click to demand the random generation of buildings, a numeric value specifying the number of buildings to be generated and another mouse click to confirm). For each virtual building, a priority-one kitchen and a living room are selected with a random order, just like in the other testing sets. Then, a set of private rooms is randomly picked (one to nine in these tests). Also, a set of bathrooms is also selected with a restriction: its number cannot be higher than the number of private rooms. Both of the selected priority-one and priority-two division instances are integrated in a list. The building format (layout's constraint shape configuration) is randomly chosen from the following ones: fully convex, L-shaped or U-shaped. Next, a node set of priority-one and priority-two divisions is mounted as a treemap structure considering the previously selected divisions list and several operations regarding pseudo-random selections and arrangements. The last step converts the graph and nodes into production grammar rules, that are passed to the procedural modelling module, responsible for the 3D model generation. Figure 7.10 depicts the results obtained by using the stochastic process developed for the generation of random virtual buildings.

\subsection{Ontology-based Architectural Derivation}

This section presents and demonstrates the buildings generation focusing the ontology derivation. To achieve it, some examples of structures extending from the generic ontology to represent elements belonging to the roman architecture were used for concept-proof purposes (both generic and roman ontology were detailed in chapter 3 ). Those structures were properly implemented in the procedural modelling module through a set of classes - following the object-oriented programming paradigm - that are used to guide the execution of the procedural modelling process. Furthermore, the geometrical definitions were also implemented for each one of those classes (instances of extension classes without geometric definitions make 


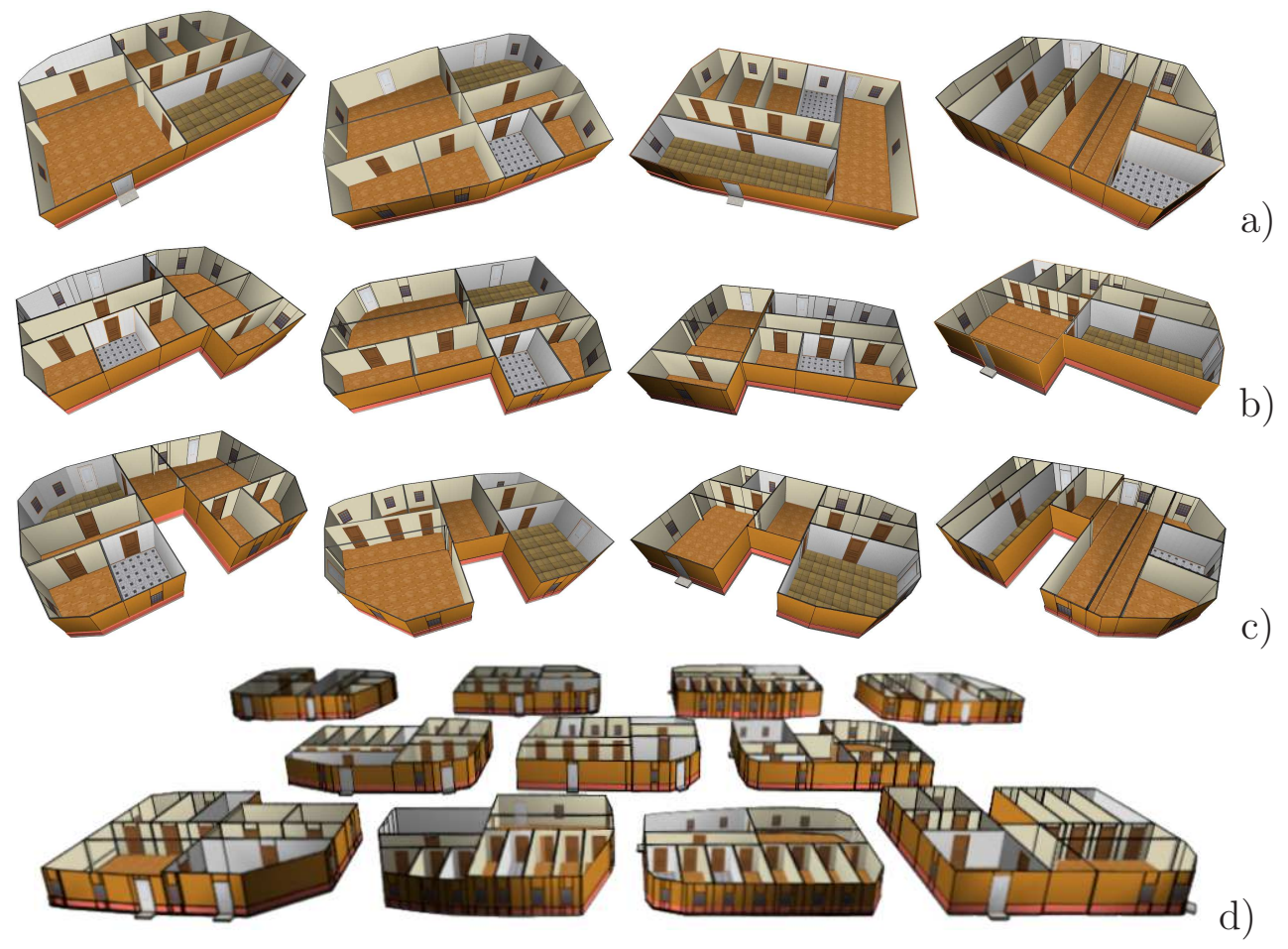

Figure 7.10 - Stochastic production of virtual buildings obtained from three fixed sets of divisions and a fourth set with randomly selected divisions. In a), b) and c) a fixed set of divisions - specifically a kitchen, a living room, three rooms and a bathroom - is used to generate several virtual buildings variants. While a) depicts the possibilities of arrangements that can result from the referred divisions set inside a fully convex constraint polygon, b) and c) present the results with the same divisions set for $L$ and $U$-shaped houses, respectively. Finally, d) exposes a subset of houses, taken from a group of twenty randomly generated buildings.

use of the geometry specified for their respective parent classes). Thus, it is possible to improve the procedural modelling module knowledge, based on the reuse of the generic ontology.

Specifically, a generic purpose building and a roman domus that resulted from the aforementioned ontological extensions. The domus overrides the generic definitions to produce notably distinct structures such as peristylium gardens or atrium pools. 


\subsubsection{Generic building and roman domus overview}

The generated virtual building (Figure 7.11) that relies on the generic ontology has a set of divisions distributed by four building parts: an entry kitchen that connects to a small corridor which, in turn, leads to a central corridor that interfaces with other two small corridors: one corridor leads to a room and the other one interfaces with another room and also with another bathroom. Regarding the domus a typical roman house-based structure was generated consisting in two main building parts: the atrium and the peristylium. The former has a water recoil system that consists in a sort of pool receiving water from a roof opening. The latter contains a big garden. Moreover, both the atrium and the peristylium can be seen as central corridors providing access to the majority of rooms inside the domus.

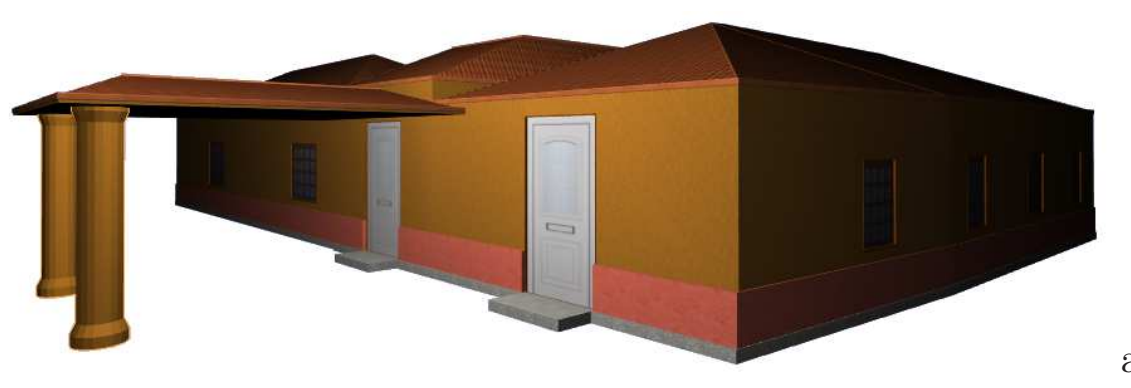

a)

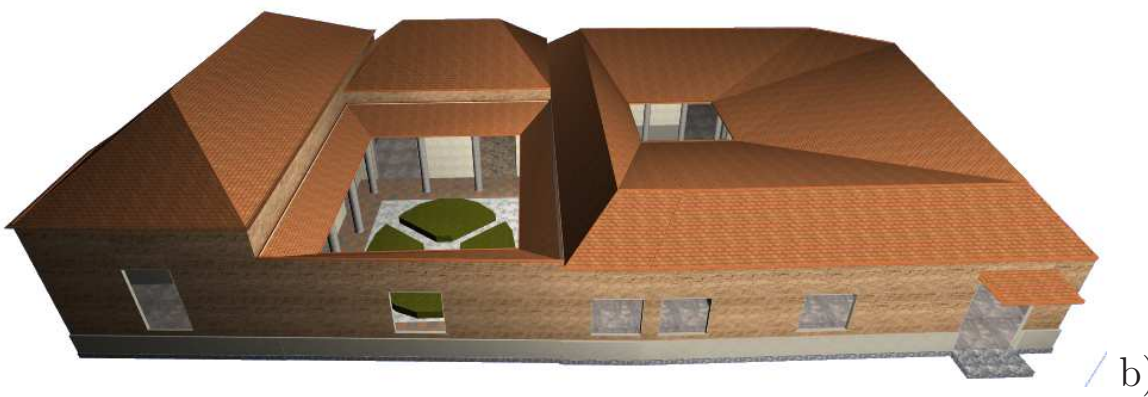

Figure 7.11 - Ontology-based generation of a generic building and a roman domus. a) depicts a generic ontology-based building; b) presents a roman ontology-based domus. A part of its peristylium can be observed through the open roof with the widest aperture, at domus left side. The other open roof with the smallest aperture is the compluvium of the building's atrium. 


\subsubsection{Structural extensions and differences}

Several differences might be noticed in the structure of the generated buildings. The generic building has transitions enclosed by doors and windows bindings in opposition to the domus, that only contains openings in transitions. Both of these buildings contain a porch and a garden with distinct geometries and representations. The gardens of both buildings consist in open-air structures, but in the specific case of the domus, a garden is also a structure surrounded by columns that belongs to a peristylium division. Moreover, the generic building porch is supported by columns whereas the domus porch is not. Additionally, the domus was enriched with a special division called atrium to adapt a compluvium/impluvium. Therefore, the procedural modelling module was instructed to produce two structures in the presence of an atrium inside a building part: an impluvium that is a Pool extended from the AbstractElement of the generic ontology and a mansard roof with a central opening called compluvium. The textures were also changed to distinguish each architectonic structure. Figure 7.12 presents the aforementioned structures pointing out the main differences.

\subsection{Performance Results}

Performance tests were made to evaluate the impact of the methodology implementation in computer resources. The evaluation considered the following parameters: average load per CPU core, RAM usage, time and number of generated vertices. The test focused in the geometry generation, rather than in the visualization and consisted in two phases: firstly, a set of buildings was generated with a progressive increment of building parts and divisions; then, this chapter's previously referred structures were subjected to the same measurements. The computer that performed the tests possesses an Intel ${ }^{(\mathrm{R})} \mathrm{Core}^{(\mathrm{TM})}$ i7 CPU (model M620), at 2.67GHz and 4GB of RAM.

The first tests consisted in generating five groups of five virtual buildings each, with a gradual increment of building parts per group and divisions per generation. The 


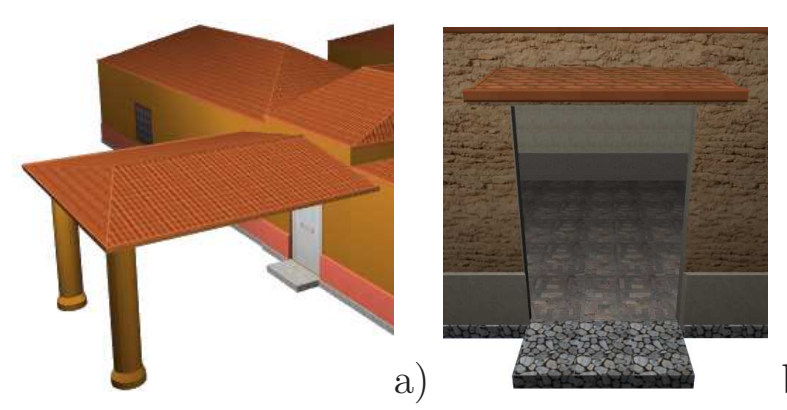

b)
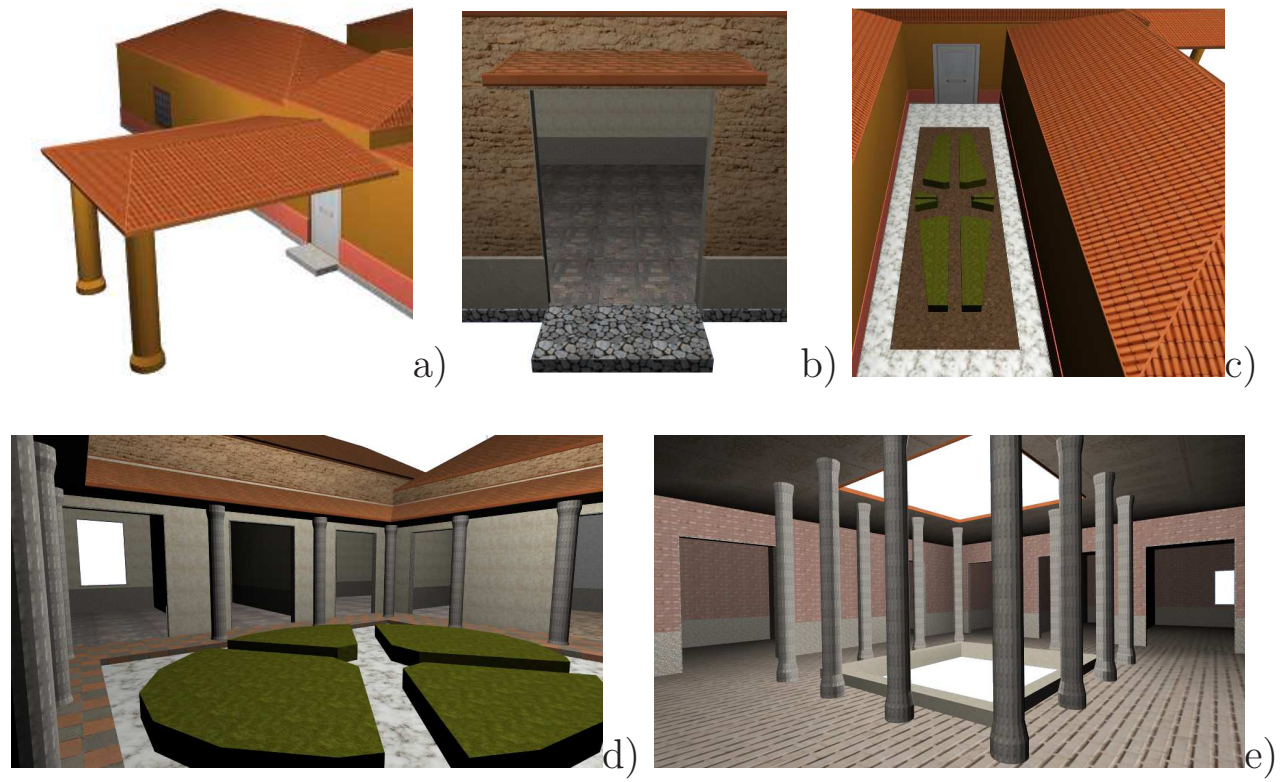

Figure 7.12 - Structural differences between the domus and the generic building: a) and b) depict the differences between the generic porch and the roman porch; c) and d) expose the differences between the generic garden and the peristylium garden; finally e) presents the compluvium and impluvium structures (used for water collection) that belong to the domus atrium.

first group has five generated virtual buildings with three, four, five, six and seven divisions organized in a single building part. The second one, contains five buildings with five, six, seven, eight and nine divisions distributed by two building parts. In the third group, five produced buildings composed by three building parts aggregating seven, eight, nine, ten and eleven divisions are presented. The penultimate set has five buildings, each one composed by four building parts that hold nine, ten, eleven, twelve and thirty divisions. Lastly, a set of five produced buildings are presented, each one having five building parts that are composed by eleven, twelve, thirteen, fourteen and fifteen divisions. The established inner transitions consisted in unique acyclic path which ensure the connectivity of the divisions inside the virtual buildings. Thus, a building with 10 divisions contains 9 transitions which is the minimum value to provide reachability among those divisions. The chosen roof for each generation trial was the flat type in order to minimize the number of 
roof vertices and to provide a fairer comparison between structures under this same condition. Plot 7.13 depicts these tests.

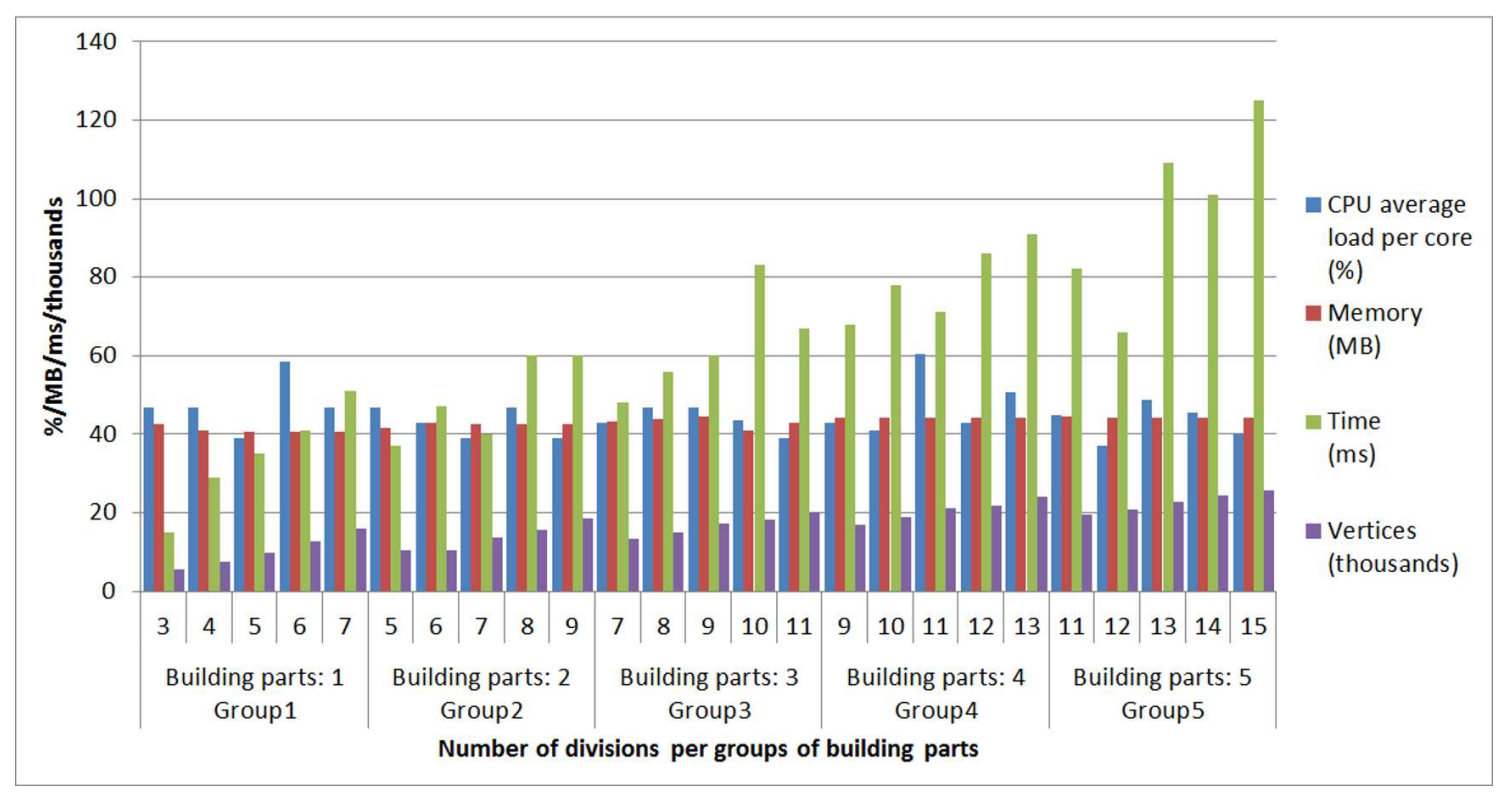

Figure 7.13 - Chart depicting the performance tests made to five groups of virtual buildings with a gradual increment of building parts per group and divisions per generation. Group1 points out the performance results corresponding to the tests made with a single building part holding three, four, five, six and seven divisions; Group2 presents the performance results considering two building parts containing five, six, seven, eight and nine divisions; the performance results regarding the generated buildings composed by three parts holding 7, 8, 9, 10 and 11 divisions are exposed in Group3; the fourth group (Group4) contains the performance results obtained by several configurations of four building parts containing 9, 10, 11, 12 and 13 divisions; finally the Group5 is underlying the plot area reserved for the results of the tests using configurations of 5 building parts, each one holding 11, 12, 13, 14 and 15 divisions.

The previous plot reveals that the average CPU usage stabilized between $37 \%$ and $61 \%$ during the tests. The interval of RAM consumption is about ]40, 45[MB. It is also notorious that the most complex structure - with 25728 vertices - took no longer than $125 \mathrm{~ms}$ to be generated. Moreover, the generation of the most basic one - composed by 5522 vertices - took $15 \mathrm{~ms}$.

The virtual buildings presented in the previous subsections were also subjected to 
the same performance tests. Results of the tests made to the buildings presented in section 7.2 to demonstrate divisions with convex delimitation through wall adaptation are depicted in figure 7.14. The first building generated has 20827 vertices and took $132 \mathrm{~ms}$. The CPU average usage per core reached $48 \%$ and the memory consumption was around 45MB. The second building is composed of 21845 vertices. Generation time reached $112 \mathrm{~ms}$ and $\mathrm{CPU}$ and memory readings revealed a consumption of $43 \%$ and $\approx 45 \mathrm{MB}$, respectively. The third building has 14821 vertices that took $111 \mathrm{~ms}$ to generate and spent $39 \%$ of $\mathrm{CPU}$ and $\approx 44 \mathrm{MB}$ of memory. The last building has resemblant values to the latter: 14988 vertices generated in $132 \mathrm{~ms}$, with $\approx 36 \%$ of $\mathrm{CPU}$ usage and $\approx 45 \mathrm{MB}$ of memory consumption.

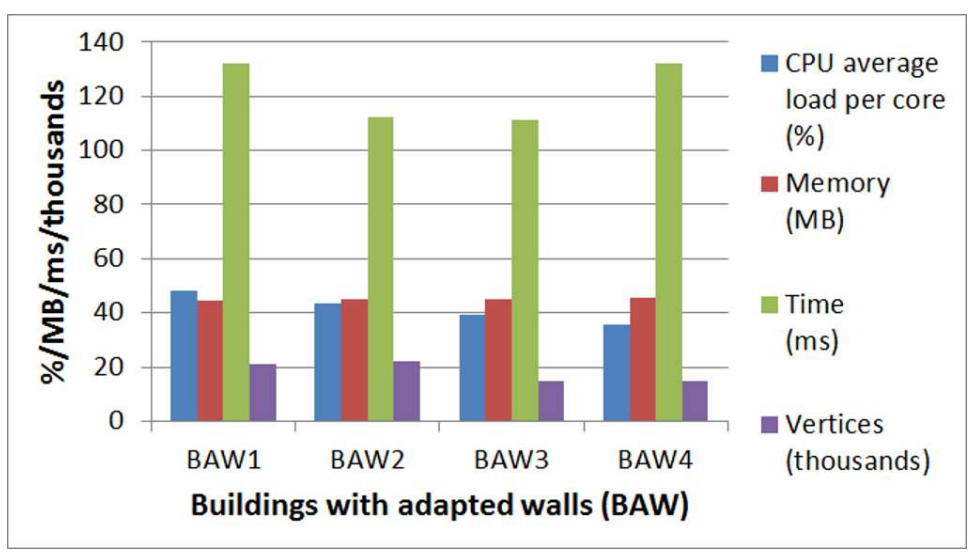

Figure 7.14 - Results of the performance tests made to measure the computational resources spent by the application of the procedural modelling methodology in the generation of virtual buildings characterized by internal walls adaptation, presented in section 7.2. The plot exposes buildings with adapted walls as BAW. BAW1 represents a building with 20827 vertices which took $132 \mathrm{~ms}$ to be generated and used $47.97 \%$ of CPU and $44.72 \mathrm{MB}$ of memory. BAW2 refers to a building composed by 21845 vertices generated in $112 \mathrm{~ms}$ with $43.29 \%$ of CPU consumption and $44.78 \mathrm{MB}$ of memory usage. BAW3 required $39.33 \%$ of $\mathrm{CPU}$ and $44.78 \mathrm{MB}$ of memory to generate its 14821 vertices in $111 \mathrm{~ms}$. Generating BAW4 took $132 \mathrm{~ms}$ to create 14988 vertices, using $45.41 \mathrm{MB}$ of RAM and $35.88 \%$ of CPU.

The subdivided structures exposed in section 7.3 were also considered for performance measurements. The first structure - which was divided in 10 divisions - used $42 \%$ of $\mathrm{CPU}$ and $\approx 42 \mathrm{MB}$ of RAM to be generated. The execution time reached 
98ms to create its 29044 vertices. The second structure, divided into 15 divisions, resulted in a set of 27108 vertices produced in $184 \mathrm{~ms}$. CPU usage was around $33 \%$ and memory allocation was above 45MB. The 15 division structure resulted in 32957 vertices generated in $179 \mathrm{~ms}$. CPU indicated $30 \%$ of average load per core and less than $45 \mathrm{MB}$ of memory usage. Lastly, the most demanding structure of this particular test took $386 \mathrm{~ms}$ to be generated and is composed of 30 divisions and 50024 vertices. However, memory and CPU measurements did not provide significant variations: $31 \%$ and $47 \mathrm{MB}$, respectively. Figure 7.15, depicts the graphical results of these performance tests.

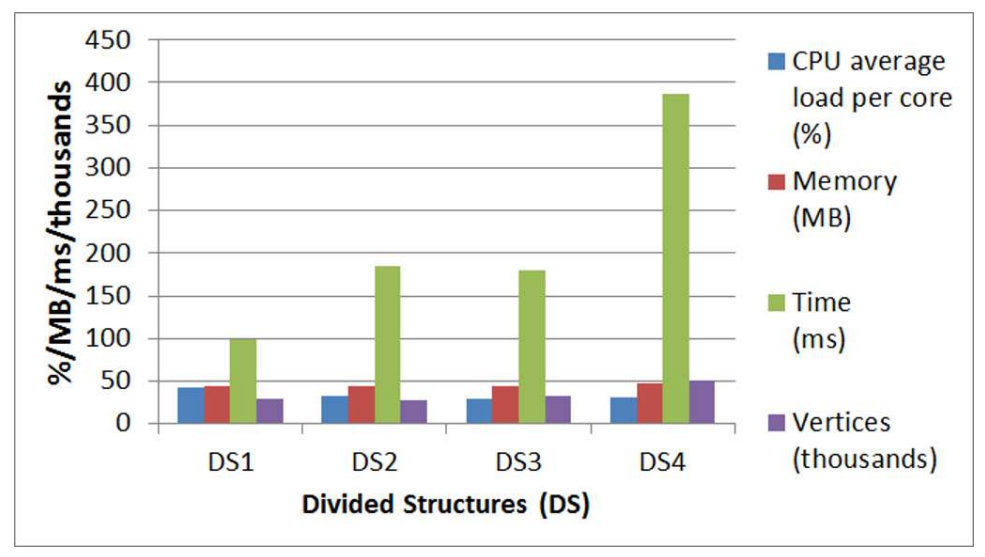

Figure 7.15 - Results of the performance tests made to the structures subdivision process presented in section 7.3. Those four structures are designated as division structures (DS) in this plot: DS1 is the lightest structure - 10 division - with the lowest generation delay (98ms). DS2 and DS3 are structures with 15 and 20 divisions, respectively, which spent similar system resources (around $31 \%$ of CPU usage and $44 \mathrm{MB}$ of memory) and time ( $\approx 180 \mathrm{~ms})$; The fourth structure - 30 divisions - took the highest time value (386ms). However, CPU (31\%) and memory $(\approx 47 \mathrm{MB}$.) utilization is not too different from the other structures.

One final performance test was made to the structures addressed in section 7.5: the general purpose building and the roman domus. The former took $139 \mathrm{~ms}$ to generate 19875 vertices, spending $44.68 \%$ of CPU (average value per core) and 44.82MB of RAM. Domus is the most complex structure, having 53443 vertices that were generated in $278 \mathrm{~ms}$, requiring $44.80 \%$ of $\mathrm{CPU}$ and $45.62 \mathrm{MB}$ of RAM. Figure 7.16 depicts this performance tests results. 


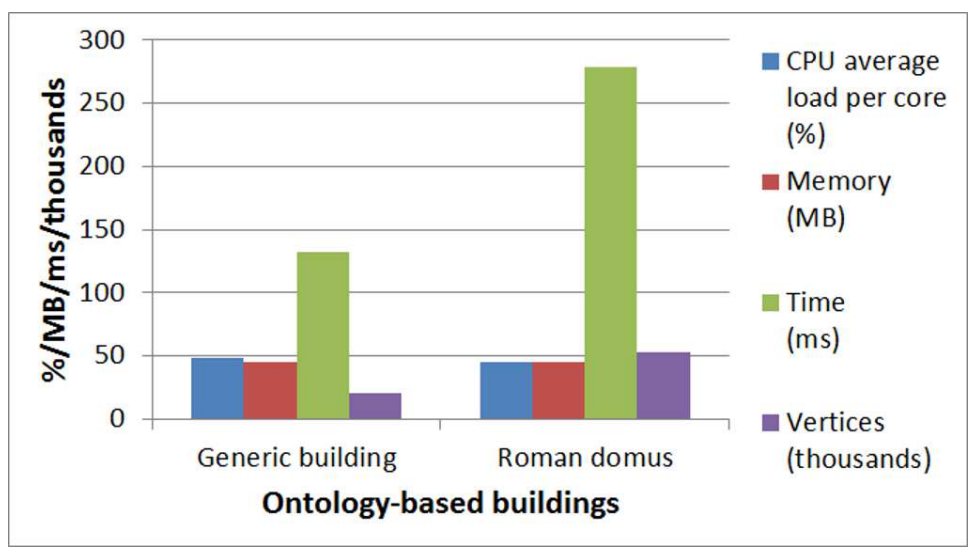

Figure 7.16 - Results of the performance tests made to the generation of ontological structures presented in section 7.5. The generic house used $\approx 45 \%$ of CPU and $\approx 45 \mathrm{MB}$ to generate 19875 vertices in $139 \mathrm{~ms}$. Domus - the most demanding structure in this tests - is constituted by 53433 vertices and was generated in $278 \mathrm{~ms}$, using $\approx 45 \%$ of CPU and $\approx 46 \mathrm{MB}$ of RAM.

\subsection{Summary}

A toolkit implementing the final version of the proposed procedural modelling methodology was used to generate virtual building models for evaluation, pointing out the methodology flexibility and validating its implementation, by producing a large variety of random structures while ensuring coherency.

Based on the presented results, it was demonstrated that this methodology is capable of producing virtual buildings confined by arbitrary convex shapes and also fake-concave shapes, using a method that flags geometric holes. This fake-concave method is appropriated for producing L-shaped or U-shaped buildings.

Moreover, the process for inner wall adaptation seems to be suitable and effective to define the number of inner wall segments that constraint a given division. There is the possibility of generating divisions containing four to eight wall segments.

The subdividing process was also tested regarding its effectiveness. Treemap was pushed to divide several buildings constrained by arbitrary outline into divisions 
and holes. It became evident that the limit for splitting will only depend on the size of the constraint polygon, number of inner divisions and related dimensions.

The predictable nature of this treemap approach allowed the development of the stochastic process for fully automatic virtual buildings production. This process was applied to generate a set of buildings with different division sets, distinct arrangements and also with different types of constraint limits: convex, U-shaped and L-shaped. The related tests highlighted the process potential in generating several buildings with random formats and aleatory division sets, autonomously. Another addressed aspect was the extensibility of the proposed generic ontology, which derived to the roman architecture to demonstrate the flexibility and adaptability of such knowledge organization in the present methodology. A general purpose building was compared to a roman domus. Without changing the methodology's core, the modeller was capable of producing virtual buildings with quite different structures. Finally, performance tests were carried out regarding the methodology implementation. It was demonstrated that simple structures with around 5500 vertices can be geometrically produced in a scarce $15 \mathrm{~ms}$, while more complex structures composed by $\approx 53000$ vertices can be generated in less than $300 \mathrm{~ms}$. 



\section{Conclusions}

This last chapter succinctly reviews the main contributions of this work, briefly compares some of its aspects with others and presents a set of notes to be considered as future research opportunities.

\subsection{Thesis Summary}

This work started with a literature review on ontologies for virtual environments and procedural modelling solutions, that allowed the identification of some issues: the apparent lack of a robust standard-based virtual building ontology, capable of, at least, mapping the majority of real-world possibilities; the insufficiency of procedural modelling methodologies dealing with traversable buildings constrained by arbitrary shapes and composed by inner divisions also arbitrarily shaped. To overcome this issues, a flexible and extensible building ontology was proposed and a procedural modelling methodology, guided by this ontology and matured throughout several development stages, was presented. 


\subsubsection{Building Ontology}

The presented ontology was designed considering the suggestions given by CityGML standard (Gröger et al., 2008, 2012) and started of as being generic. That knowledge structure established that the Building is the major abstract container which is composed by one or more horizontal compositions displaced in the building's ground, also known as building parts. Every building part if formed by one or more floors, each one containing the interior divisions. The boundary surfaces represent the walls that delimit entities, such as divisions (interior isolation) and floors (exterior isolation). Finally, transitions representing the traversable points and other types of boundary openings - like doors and windows - are directly related to the building and abstract building elements (divisions and floors).

Afterwards, the roman architecture was used to demonstrate how to derive the generic ontology. The main knowledge structure is always maintained, while extending the elements into particular classes. For example, a Building extends to RomanBuilding which, in turn, can be extended to a Domus, Theater, Forum and so on. Moreover, particular building parts or divisions can extend from the generic BuildingPart or Division, respectively. For example Peristylium, Culina and Cubiculum are roman rooms that inherit features from the generic Division. The $A b$ stractElement is an ontological entity planned to support the extension to concrete structures. In the case of the roman architecture, arches and columns are represented as inherited structures from the generic AbstractElement class. Every other architectonic style can be described through the same extension mechanism.

Besides the structuring of building elements, the ontology also performs an important role in the procedural modelling methodology regulation, summed up in the following subsection.

\subsubsection{Procedural Modelling Methodology}

The generic procedural modelling methodology for the generation of virtual buildings was present since the beginning of this thesis. According to it, a virtual building 
model is achieved by: firstly, determine a floor plan; then, connected divisions; afterwards, extrude the walls, generate the roof and create the final elements, such as footers and transitions. However, this generic methodology progressed from chapter to chapter, as it was being enhanced and improved. In fact, while the earlier chapters presented a process capable of producing virtual buildings only with square geometries, further on it was already possible to subdivide constraint convex polygons and to define geometric holes (fake-concave constraint polygons). Another improvement was the possibility of defining the number of inner wall segments of a division which implicitly assumes a shape based on this definition. Moreover, the flexibility of the presented procedural modelling methodology was demonstrated by generating two types of virtual structures (generic and roman). A stochastic process which takes advantage from the predictability and flexibility of the floor plan subdivision method - the treemap approach - was also proposed to demonstrate that the current methodology can also be used to produce buildings quickly and considering a minimalist set of inputs. So, summing up the different phases of the procedural modelling methodology:

\section{A) Floor plan}

The floor plan subdivision method relies in a treemap approach. The recursion attached to the method allowed the splitting of constraint building polygons into smaller areas to provide the proper division arrangements. Originally, treemap was used to deal with rectangles but it also proved effective in dividing other convex shapes beyond the rectangles. The generation of floor plans in this procedural modelling methodology also supports the adjustment of room shapes through the modification of delimiting wall segments.

B) 3D virtual model

The 3D models are achieved through a set of steps, applied after the floor plan subdivision: the inner walls and the facade walls are extruded to a given height; transitions, specifically doors and windows, are subjected to a similar extrusion process; each building part is then covered with a roof; and finally some model enhancements are made, such as the creation of footers, frames for windows and 
doors and thickening the roof skeleton. The resulting virtual buildings models provide a coherent visualization that allows to clearly distinguish the different building elements and architectonic style features. Virtual building models can be further enriched with the proper ontological extensions and geometrical implementations.

One final remark goes to the possibility of using the proposed procedural modelling methodology for generating buildings, deterministic and stochastically. This methodology is mainly oriented for the deterministic generation of structures. The rules that feed the generation through XML or grammar are rigid values that do not consider alternatives for the building production, i.e., the same rules with the same parameters will always produce one single structure. However, the manageability of the methodology - namely at the treemap level approach - along with pseudo-random attributions for division arrangements, building part heights and other grammar parameters allowed the establishment of a stochastic process, thus enabling the use of this methodology for the fully automatic generation of buildings.

\subsection{Discussion and Future Work}

A wide variety of solutions regarding the procedural modelling of virtual buildings with interiors has been proposed by some authors, as for example Martin (2005), Hahn et al. (2006), Merrell et al. (2010), Marson and Musse (2010) and Rodrigues (2010) with interesting automation processes (e.g. computer-generated floor plans) and results. However, most of them confine the building's geometries to squares. Other works, such as those proposed by Hohmann et al. (2010) and Leblanc et al. (2011) opted for using CSG algorithms and generative modelling languages to explore their great potential to generate complex structures. Those approaches, which require programming skills and are usually very time-consuming, were also applied to produce traversable buildings, mainly composed by rectangular shapes. Regarding the shapes' arbitrariness, only the work developed by Rinde (2008) was found during this thesis literature review. This author proposed a solution for generating building structures constrained by non-squares geometric shapes. However, the 
interior divisions' generation carried out by the algorithm does not seem to be appropriate when rooms' generation control (for example geometric constraints and rooms' arrangements) is required. Rinde (2008) also documents the impossibility of dealing with building limits containing geometric holes. In addition, texturing is an absent feature, making impossible the visual distinction between room types.

Moreover, despite the straight orientation of CityGML for urban environments, the standard remains almost unused in procedural modelling works. Ontology-based solutions work with customized semantics or ontologies. Since CityGML constitutes the standard for virtual urban environments validated and released by a consortium, it was preferred as a base for the creation of the regulatory ontology for buildings that intends to fit a wider range of structures present in real world.

Considering the aforementioned, a new ontology-based procedural modelling methodology was proposed resulting in a set of achievements:

- the proposed methodology is capable of producing traversable buildings constrained by arbitrary convex shapes;

- a process to change the format of the interior rooms was achieved through the wall number modification;

- a "fake-concave" technique was properly adapted to support non-convex buildings layouts;

- an extensible CityGML-based building ontology to guide the procedural modelling process and to support the generation of other architectural style buildings (e.g. roman houses) was designed and implemented within the methodology context;

- some ontology-based structures - data model and grammar - were proposed to establish input formats (definition rules institution);

- finally, a computer managed processes regarding the stochastic generation was implemented to automatize buildings' production. 
A procedural modelling methodology evaluation was carried out to attest the accomplishments listed above. Summing up, the proposed procedural modelling methodology complies with the objectives and main contributions that were defined for this thesis. As future work, further enhancements and improvements were identified and can be done to the presented procedural modelling methodology, namely:

- Support to multiple floors: the flexibility of the proposed ontology foresees multi-floor buildings. However, the proposed procedural modelling methodology requires a few modifications to support the generation of buildings with more than a ground floor. At least, a workaround can be achieved based on the establishment of some assumptions, for example:

- in multi-floor buildings, usually only the ground floor is a different from all others while the remaining ones are replicas from the first floor;

- the different floors are connected through a common aligned element which provide access to the different levels (an elevator or a staircase).

- Support to pure concave geometries: currently, the fake-concave technique is enough to produce buildings with geometric holes. However, a pure concave approach (e.g. ear-clipping) could be an interesting comparison feature;

- Reuse of divisions' dead zones: division wall's adaptation results in dead zones (or blank zones) that currently contribute to the waste of buildings' useful area. To address this issue, some resolutions can be considered. A possibility could pass by re-injecting the wasted area of those dead zones into the divisions that borrowed spaces during the floor plan probing stage. One might also consider the relaxation of some restrictions to adapt a building's constraint shape to the divisions' shapes with a goal of removing those death zones or even to arrange the divisions' walls segments in a more advantageous format aiming the reduction of space waste;

- Development of a tool for geometric customization: the geometric kit developed for the proposed methodology is extensive and flexible. However, the production of geometry for each ontological object is programmer-dependent. 
The integration of a GUI-based tool for customizing shapes would enable this independence, passing the appearance responsibilities to the designers and final users;

- Inclusion of furniture: the ontology enables the differentiation between divisions which can constitute a good starting point for the automatic placement of furniture, according to the division types;

- Support to direct user interaction with the generated virtual models: currently, only the automatic modelling of buildings is regarded to create virtual models for visualization. Enabling user interaction could improve the supporting system which would be able to adapt user modifications upon the 3D model interactively.

\subsection{Final Remarks}

The accomplishment of this work has been a rewarding journey that helped me to better understand the field of Computer Graphics and Procedural Modelling. But, more important than that, it helped me to mature the researching process.

Another important lesson that was taken during the development of this work is that knowledge is not everything. Albert Einstein stated once that "Logic will get you from A to B; Imagination will take you everywhere". Thus, creativity might also constitute a powerful mind tool to identify issues and overcome challenges.

There is still a lot of remaining work to do because, accordingly with which was said by the renowned dramatist George Bernard Shaw "Science never solves a problem without creating ten more". However, it is hoped that this thesis constitutes a valuable contribution to the improvement of Procedural Modelling field, opening doors for new processes, methods and techniques. 



\section{References}

Adão, T., Magalhães, L., Bessa, M., Coelho, A., Sousa, A., Rodrigues, N., Rodrigues, R., Pereira, F., Moura, J., and Reis, L. (2012). Eras - an ontology-based tool for the expeditious reconstruction of virtual cultural heritage sites. Actas do 20 Encontro Português de Computação Gráfica, Viana do Castelo, Portugal. 55

Adão, T., Batista, R., Peres, E., Magalhães, L. G., and António, C. (2013a). Reconstructing traversable buildings for archaeology with eras. In Proc. Virtual and Networked Organizations: Emergent Technologies and Tools - ViNOrg '13. 89, 113

Adão, T., Magalhães, L., and Peres, E. (2013b). Semi-automatic virtual reconstruction of ancient roman houses. In Information Systems and Technologies (CISTI), 2013 8th Iberian Conference on, pages 1-6. 69

Adão, T., Magalhães, L., Peres, E., and Pereira, F. (2014). Procedural generation of traversable buildings outlined by arbitrary convex shapes. Procedia Technology, 16(0):310 - 321. CENTERIS 2014 - Conference on ENTERprise Information Systems. 89

Aichholzer, O. and Aurenhammer, F. (1996). Straight skeletons for general polygonal figures in the plane. pages 117-126. Springer-Verlag. 25 
Attene, M., Robbiano, F., Spagnuolo, M., and Falcidieno, B. (2009). Characterization of $3 \mathrm{~d}$ shape parts for semantic annotation. Computer-Aided Design, 41(10):756-763. 5, 15, 17

Azhar, S. (2011). Building information modeling (bim): Trends, benefits, risks, and challenges for the aec industry. Leadership and Management in Engineering, $11(3): 241-252.5$

Bellotti, F., Berta, R., Cardona, R., and De Gloria, A. (2011). An architectural approach to efficient 3d urban modeling. Computers \& Graphics, 35(5):10011012. 22,34

Benjamins, V. R. and Fensel, D. (1998). The ontological engineering initiative (ka)2. 14

Besuievsky, G. and Patow, G. (2013). Procedural modeling historical buildings for serious games. Virtual Archaeology Review, 4(9):160 - 166. 28

Béhé, F., Galland, S., Gaud, N., Nicolle, C., and Koukam, A. (2014). An ontologybased metamodel for multiagent-based simulations. Simulation Modelling Practice and Theory, 40(0):64 - 85. 15, 18, 56

Borgo, S., Guarino, N., and Masolo, C. (1997). An ontological theory of physical objects. In Proceedings of Qualitative Reasoning 11th International Workshop, pages 223-231. 14

Bradley, B. (2005). Towards the procedural generation of urban building interiors. PhD thesis, M. Sc. thesis, Game Programming, University of Hull. 37, 49, 52

Bruls, M., Huizing, K., and van Wijk, J. (1999). Squarified treemaps. In In Proceedings of the Joint Eurographics and IEEE TCVG Symposium on Visualization, pages 33-42. Press. xxiv, 40, 41, 57

BuildingSMART (2013). Industry foundation classes release 4. http://www. buildingsmart-tech.org/ifc/IFC4/final/html/. Accessed: May, 2015. 22 
Carrozzino, M., Tecchia, F., and Bergamasco, M. (2009). Urban procedural modeling for real-time rendering. In Proceedings of the 3rd ISPRS International Workshop 3D-ARCH, volume 2009. 13, 31

Chandrasekaran, B., Josephson, J., and Benjamins, V. (1999). What are ontologies, and why do we need them? Intelligent Systems and their Applications, IEEE, 14(1):20-26. 14, 15

Coelho, A., Bessa, M., Sousa, A. A., and Ferreira, F. N. (2007). Expeditious modelling of virtual urban environments with geospatial l-systems. In Computer Graphics Forum, volume 26, pages 769-782. Wiley Online Library. 23

Cohen, K. (2001). Monsters, inc.: The secret behind why pixar is so good. Animation World Magazine, (6):6-12. 4

Colledani, M., Pedrielli, G., Terkaj, W., and Urgo, M. (2013). Integrated virtual platform for manufacturing systems design. Procedia CIRP, 7:425-430. 18

Correia, V. (2002). Conimbriga ruínas - museu monográfico. http://www . conimbriga.pt/portugues/ruinas0.html. Accessed: January, 2014. 113

Dikaiakou, M., Efthymiou, A., and Chrysanthou, Y. (2003). Modelling the walled city of nicosia. In Proceedings of the 4th International Conference on Virtual Reality, Archaeology and Intelligent Cultural Heritage, VAST'03, pages 61-70, Aire-la-Ville, Switzerland, Switzerland. Eurographics Association. 25

Dylla, K., Frischer, B., Müller, P., Ulmer, A., and Haegler, S. (2010). Rome reborn 2.0: A case study of virtual city reconstruction using procedural modeling techniques. In 37th Proceedings of the CAA Conference, pages 62-66. EG. 3, 4

Dylla, K., Muller, P., Ulmer, A., Haegler, S., and Fischer, B. (2009). Rome reborn 2.0: A framework for virtual city reconstruction using procedural modeling techniques. In Proceedings of Computer Applications and Quantitative Methods in Archaeology. xxiii, 28, 29, 56 
Eppstein, D. and Erickson, J. (1998). Raising roofs, crashing cycles, and playing pool: Applications of a data structure for finding pairwise interactions. In Proceedings of the Fourteenth Annual Symposium on Computational Geometry, SCG '98, pages 58-67, New York, NY, USA. ACM. 24

Felkel, P. and Obdrzalek, S. (1998). Straight skeleton implementation. In Proceedings of spring conference on computer graphics. 24, 25

Finkenzeller, D. (2008). Detailed building facades. Computer Graphics and Applications, IEEE, 28(3):58-66. 27

Finkenzeller, D., Bender, J., and Schmitt, A. (2005). Feature-based decomposition of façades. In Proc. Virtual Concept. 26

Finkenzeller, D. and Schmitt, A. (2006). Rapid modeling of complex building façades. Institut für Betriebs-und Dialogsysteme, Universität Karlsruhe (TH), Germany. 26

Flotynski, J. (2014). Semantic modelling of interactive 3d content with domainspecific ontologies. Procedia Computer Science, 35(0):531 - 540. Knowledge-Based and Intelligent Information \& Engineering Systems 18th Annual Conference, KES2014 Gdynia, Poland, September 2014 Proceedings. 18

Galambos, P., Ádám Csapó, Zentay, P., Fülöp, I. M., Haidegger, T., Baranyi, P., and Rudas, I. J. (2014). Design, programming and orchestration of heterogeneous manufacturing systems through vr-powered remote collaboration. Robotics and Computer-Integrated Manufacturing, (0):-. 19

García-Rojas, A., Gutiérrez, M., and Thalmann, D. (2008). Visual creation of inhabited 3d environments. The Visual Computer, 24(7-9):719-726. 16

Greuter, S., Parker, J., Stewart, N., and Leach, G. (2003). Real-time procedural generation of 'pseudo infinite' cities. In Proceedings of the 1st International Conference on Computer Graphics and Interactive Techniques in Australasia and South East Asia, GRAPHITE '03, pages 87-ff, New York, NY, USA. ACM. xxiii, 25,26 
Gröger, G., Kolbe, T. H., Czerwinski, A., and Nagel, C. (2008). OGC City Geography Markup Language(CityGML) Encoding Standard. Technical report, Open Geospatial Consortium. 2, 6, 9, 19, 56, 59, 172

Gröger, G., Kolbe, T. H., Nagel, C., and Häfele, K.-H. (2012). OGC City Geography Markup Language(CityGML) Encoding Standard. Technical report, Open Geospatial Consortium. 2, 6, 9, 19, 56, 59, 172

Guarino, N. (1998). Formal ontology and information systems. In Proceedings of the first international conference (FOIS'98), pages 3-15. IOS Press. 14, 15

Hahn, E., Bose, P., and Whitehead, A. (2006). Lazy generation of building interiors in realtime. In Electrical and Computer Engineering, 2006. CCECE '06. Canadian Conference on, pages 2441 -2444. xxiii, 13, 37, 38, 49, 57, 174

Havemann, S. (2005). Generative Mesh Modeling. PhD thesis. 43

Hohmann, B., Havemann, S., Krispel, U., and Fellner, D. (2010). A gml shape grammar for semantically enriched 3d building models. Computers $\&$ Graphics, 34(4):322 - 334. Procedural Methods in Computer Graphics Illustrative Visualization. $43,51,53,57,123,174$

Hunter, J. and Gerber, A. (2010). Harvesting community annotations on 3d models of museum artefacts to enhance knowledge, discovery and re-use. Journal of Cultural Heritage, 11(1):81-90. 5, 17

Jannin, P. and Morandi, X. (2007). Surgical models for computer-assisted neurosurgery. Neuroimage, 37(3):783-791. 15, 16

Johnson, B. and Shneiderman, B. (1991). Treemaps: A space-filling approach to the visualization of hierarchical information structures. In Visualization, 1991. Visualization'91, Proceedings., IEEE Conference on, pages 284-291. IEEE. 58, 123

Kolbe, T. H. (2009). Representing and exchanging 3d city models with citygml. In $3 D$ geo-information sciences, pages 15-31. Springer. xxiii, 20, 21 
Kolbe, T. H. (2012). What is citygml? 6

Koutsourakis, P., Simon, L., Teboul, O., Tziritas, G., and Paragios, N. (2009). Single view reconstruction using shape grammars for urban environments. In Computer Vision, 2009 IEEE 12th International Conference on, pages 1795-1802. IEEE. 30

Larive, M. and Gaildrat, V. (2006). Wall grammar for building generation. In Proceedings of the 4th International Conference on Computer Graphics and Interactive Techniques in Australasia and Southeast Asia, GRAPHITE '06, pages 429-437, New York, NY, USA. ACM. 24, 123

Leblanc, L., Houle, J., and Poulin, P. (2011). Component-based modeling of complete buildings. In Proceedings of Graphics Interface 2011, GI '11, pages 87-94, School of Computer Science, University of Waterloo, Waterloo, Ontario, Canada. Canadian Human-Computer Communications Society. 44, 51, 53, 57, 174

Lee, J. Y., Seo, D. W., and Rhee, G. (2008a). Visualization and interaction of pervasive services using context-aware augmented reality. Expert Systems with Applications, 35(4):1873 - 1882. 5, 16

Lee, L., Pavlov, N., and Animation, D. (2008b). Procedural fracturing and debris generation for kung-fu panda. In ACM SIGGRAPH 2008 talks, page 59. ACM. 4

Lewis, R. and Séquin, C. (1998). Generation of 3d building models from 2d architectural plans. Computer-Aided Design, 30(10):765 - 779. 45, 52

Liebich, T. (2013). Ifc4-the new buildingsmart standard. 21

Lindenmayer, A. (1968). Mathematical models for cellular interactions in development ii. simple and branching filaments with two-sided inputs. Journal of Theoretical Biology, 18(3):300 - 315. 22, 123

Liu, Y., Xu, C., Pan, Z., and Pan, Y. (2006). Semantic modeling for ancient architecture of digital heritage. Computers $\&$ Graphics, 30(5):800 - 814. 13, 33, 34 
Liu, Y., Xu, C., Zhang, Q., and Pan, Y. (2008). The smart architect: Scalable ontology-based modeling of ancient chinese architectures. Intelligent Systems, IEEE, 23(1):49-56. 5, 13, 22, 33

Liu, Y., Zheng, X., Tang, F., and Chen, X. (2014). Ontology design with a granular approach. Expert Systems with Applications, 41(10):4867 - 4877. 22, 34

Long, J. H. (2011). Room and Feature Placement for Procedurally Generated Indoor Environments. PhD thesis, University of Abertay Dundee. 36

Lopes, R., Tim, T., Smelik, R. M., Jan de Kraker, K., and Bidarra, R. (2010). A constrained growth method for procedural floor plan generation. In GAMEON'10. $42,47,51,52,57,137$

Maciel, M. (2006). Vitrúvio - Tratado De Arquitetura. Ist Press. 39, 53, 56

Marson, F. and Musse, S. R. (2010). Automatic real-time generation of floor plans based on squarified treemaps algorithm. Int. J. Comput. Games Technol., 2010:7:1-7:10. 4, 13, 40, 43, 50, 53, 57, 137, 174

Martin, J. (2005). Algorithmic beauty of buildings methods for procedural building generation. Computer Science Honors Theses, page 4. 35, 49, 57, 174

Martin, J. (2006). Procedural house generation: A method for dynamically generating floor plans. In Symposium on Interactive 3D Graphics and Games. xxiii, 36,52

Martins, T. M. P. (2011). Ontologia urbana para ambiente virtual colaborativo no contexto do planeamento e gestao municipais. Master's thesis, Faculdade de Engenharia da Universidade do Porto. 17

Merrell, P., Schkufza, E., and Koltun, V. (2010). Computer-generated residential building layouts. ACM Trans. Graph., 29(6):181:1-181:12. xxiv, 4, 13, 41, 42, 51, 57,174

Mignard, C. and Nicolle, C. (2014). Merging bim and gis using ontologies application to urban facility management in active3d. Computers in Industry, 65(9):1276 - 
1290. Special Issue on The Role of Ontologies in Future Web-based Industrial Enterprises. 19, 56

Mirahmadi, M. and Shami, A. (2012). A novel algorithm for real-time procedural generation of building floor plans. CoRR, abs/1211.5842. 40, 41, 50, 53, 57

Müller, P., Vereenooghe, T., Wonka, P., Paap, I., and Gool, L. V. (2006a). Procedural 3d reconstruction of puuc buildings in xkipché. In Eurographics Symposium on Virtual Reality, Archaeology and Cultural Heritage (VAST), pages 139-146. EG. 3, 28

Müller, P., Wonka, P., Haegler, S., Ulmer, A., and Van Gool, L. (2006b). Procedural modeling of buildings. ACM Trans. Graph., 25(3):614-623. 4, 13, 27, 28, 123

Müller, P., Zeng, G., Wonka, P., and Van Gool, L. (2007). Image-based procedural modeling of facades. In ACM Transactions on Graphics (TOG), volume 26, page 85. ACM. 30

Noy, N. F. and Hafner, C. D. (1997). The state of the art in ontology design: A survey and comparative review. AI magazine, 18(3):53. 14

Parish, Y. I. H. and Müller, P. (2001). Procedural modeling of cities. In Proceedings of the 28th annual conference on Computer graphics and interactive techniques, SIGGRAPH '01, pages 301-308, New York, NY, USA. ACM. 4, 13, 22, 23

Pittarello, F. and De Faveri, A. (2006). Semantic description of 3d environments: a proposal based on web standards. In Proceedings of the eleventh international conference on 3D web technology, pages 85-95. ACM. 15

Rau-Chaplin, A., MacKay-Lyons, B., and Spierenburg, P. (1996). The lahave house project: Towards and automated architectural design service. In Proceedings of the International Conference on Computer Aided Design (CADEX-96), pages 6266. IEEE. 35, 49, 52, 57, 123

RGEU (2008). Regulamento geral das edificações urbanas, decreto n. ${ }^{\circ}$ 38382. 39, 53 
Rinde, L. (2008). Procedural generation of indoor environments. Master's thesis, Charmers University of Technology. xxiii, 38, 39, 50, 53, 57, 174, 175

Rodrigues, N. (2010). Rule-based Generation of virtual traversable architecturalperiod houses. PhD thesis, University of Trás-os-Montes e Alto Douro. xxiii, 40, $50,53,57,174$

Rodrigues, N., Dionísio, M., Gonçalves, A., Magalhães, L. G., Moura, J. P., and Chalmers, A. (2008a). Incorporating legal rules on procedural house generation. In Proceedings of the 24th Spring Conference on Computer Graphics, SCCG '08, pages 59-66, New York, NY, USA. ACM. 39, 56

Rodrigues, N., Dionísio, M., Gonçalves, A., Magalhães, L. M. G., and P, M. J. (2008b). Rule-based generation of houses. Computer Graphics $\&$ Geometry, $10(2): 49-65.3,4,39,56$

Rodrigues, N., Magalhães, L. G., Moura, J. P., and Chalmers, A. (2008c). Automatic reconstruction of virtual heritage sites. In Proceedings of the 9th International conference on Virtual Reality, Archaeology and Cultural Heritage, pages 39-46. Eurographics Association. 39, 123

Rodrigues, N., Magalhães, L., Moura, J., Chalmers, A., Santos, F., and Morgado, L. (2010). Archhousegenerator - a framework for house generation. Journal For Virtual Worlds Research, 2(5). 40

Santos, D. S., Dionísio, M., Rodrigues, N., and Pereira, A. (2011). Efficient creation of $3 \mathrm{~d}$ models from buildingsfloor plans. International Journal of Interactive Worlds, 2011:1 - 30. xxiv, 45, 46, 52

Silva, P. B. (2010). Modelação procedimental para desenvolvimento de jogos de computador. Master's thesis, Faculdade de Engenharia da Universidade do Porto. 32

Silva, P. B. and Coelho, A. (2011). Procedural modeling for realistic virtual worlds development. Journal For Virtual Worlds Research, 4(1). xxiii, 4, 13, 32, 33 
Simon, L. (2011). Procedural reconstruction of buildings : towards large scale automatic $3 D$ modeling of urban environments. Theses, Ecole Centrale Paris. 31

Simon, L., Teboul, O., Koutsourakis, P., and Paragios, N. (2011). Random exploration of the procedural space for single-view $3 \mathrm{~d}$ modeling of buildings. International journal of computer vision, 93(2):253-271. 31

Sipser, M. (1996). Introduction to the Theory of Computation. Course Technology, Boston. 27

Sugihara, K. and Hayashi, Y. (2008). Automatic generation of 3d building models with multiple roofs. Tsinghua Science \& Technology, 13:368 - 374. 31

Tepavčević, B. and Stojaković, V. (2013). Procedural modeling in architecture based on statistical and fuzzy inference. Automation in Construction, 35(0):329 - 337. 28,29

Trescak, T., Esteva, M., and Rodriguez, I. (2010). A virtual world grammar for automatic generation of virtual worlds. The Visual Computer, 26(6-8):521-531. xxiv, $5,13,22,46,48,52,123$

Tutenel, T., Smelik, R., Lopes, R., de Kraker, K., and Bidarra, R. (2011). Generating consistent buildings: A semantic approach for integrating procedural techniques. Computational Intelligence and AI in Games, IEEE Transactions on, 3(3):274288. $4,5,47,52,57$

van Heijst, G., Schreiber, A., and Wielinga, B. (1997). Using explicit ontologies in kbs development. International Journal of Human-Computer Studies, 46(2):183 $-292.14$

Wand, Y. and Weber, R. (1990). An ontological model of an information system. Software Engineering, IEEE Transactions on, 16(11):1282-1292. 14

Watson, B., Muller, P., Wonka, P., Sexton, C., Veryovka, O., and Fuller, A. (2008). Procedural urban modeling in practice. Computer Graphics and Applications, IEEE, 28(3):18-26. 3 
Weber, R. and Lybrand, C. . (1997). Ontological Foundations of Information Systems. Coopers \& Lybrand accounting research methodology monograph. Coopers \& Lybrand and the Accounting Association of Australia and New Zealand. 14

Wonka, P., Wimmer, M., Sillion, F., and Ribarsky, W. (2003). Instant architecture. ACM Trans. Graph., 22(3):669-677. xxiii, 13, 23, 24, 123

Yong, L., Mingmin, Z., Yunliang, J., and Haiying, Z. (2012). Improving procedural modeling with semantics in digital architectural heritage. Computers $\&$ Graphics, 36(3):178 - 184. Novel Applications of VR. 22, 34 